\title{
Small molecules, big consequences
}

Citation for published version (APA):

Yadati, T. (2021). Small molecules, big consequences: novel therapeutic approaches for treating chronic inflammatory diseases. [Doctoral Thesis, Maastricht University]. Maastricht University. https://doi.org/10.26481/dis.20211118ty

Document status and date:

Published: 01/01/2021

DOI:

10.26481/dis.20211118ty

Document Version:

Publisher's PDF, also known as Version of record

\section{Please check the document version of this publication:}

- A submitted manuscript is the version of the article upon submission and before peer-review. There can be important differences between the submitted version and the official published version of record.

People interested in the research are advised to contact the author for the final version of the publication, or visit the DOI to the publisher's website.

- The final author version and the galley proof are versions of the publication after peer review.

- The final published version features the final layout of the paper including the volume, issue and page numbers.

Link to publication

\footnotetext{
General rights rights.

- You may freely distribute the URL identifying the publication in the public portal. please follow below link for the End User Agreement:

www.umlib.nl/taverne-license

Take down policy

If you believe that this document breaches copyright please contact us at:

repository@maastrichtuniversity.nl

providing details and we will investigate your claim.
}

Copyright and moral rights for the publications made accessible in the public portal are retained by the authors and/or other copyright owners and it is a condition of accessing publications that users recognise and abide by the legal requirements associated with these

- Users may download and print one copy of any publication from the public portal for the purpose of private study or research.

- You may not further distribute the material or use it for any profit-making activity or commercial gain

If the publication is distributed under the terms of Article $25 \mathrm{fa}$ of the Dutch Copyright Act, indicated by the "Taverne" license above, 
Small molecules, big consequences:

Novel therapeutic approaches for treating chronic inflammatory disorders

Tulasi Yadati 
(C) Tulasi Yadati, Maastricht 2021

All rights reserved. No part of this thesis may be reproduced or transmitted in any form or by any means, electronic or mechanical, including photocopying, recording or any information storage or retrieval system, without written permission from the author.

ISBN: 978-94-6419-330-5

Cover design: Tulasi Yadati

Thesis layout: Tulasi Yadati

Printed by: Glideprint Enschede, www.glideprint.nl

Printing of this thesis was financially supported by the

Dutch association for hepatology (Nederlandse Vereniging voor Hepatologie).

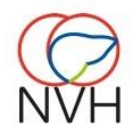

The studies presented in this thesis were performed within NUTRIM School of Nutrition and Translational Research in Metabolism. 


\section{Small molecules, big consequences:}

\section{Novel therapeutic approaches for treating chronic inflammatory disorders}

\section{DISSERTATION}

to obtain the degree of Doctor at Maastricht University,

on the authority of the Rector Magnificus Prof. dr. Rianne M. Letschert, in accordance with the decision of the Board of Deans, to be defended in public on

Thursday 18th of November 2021, at 10.00 hours

by

Tulasi Yadati 


\section{Promotor}

Prof. dr. Ronit Shiri-Sverdlov

\section{Co-promotor}

Dr. Tom Houben

\section{Assessment committee}

Prof. dr. M. Honing (Chair)

Prof. dr. M.C.G.J. Brouwers

Dr. L.B. Creemers (Utrecht UMC)

Dr. J. Theys

Dr. J. Verbeek (KU Leuven, Belgium) 


\section{Contents}

$\begin{array}{lll}\text { Chapter } 1 & \text { General introduction } & 7\end{array}$

Chapter 2 The Ins and Outs of Cathepsins: Physiological Function and Role in Disease Management

Chapter $4 \quad$ Inhibition of extracellular cathepsin D reduces hepatic inflammation in NASH mice

Chapter 5 Inflammatory Bowel Disease: A Stressed "Gut/Feeling"

Chapter 6 Pro-inflammatory implications of 2-hydroxypropyl- $\beta$ cyclodextrin treatment 



\section{Chapter 1}

General introduction 


\section{Inflammatory responses and inflammation-associated diseases}

Inflammation is a normal physiological reaction of the body to harmful stimuli, be they physical, chemical, or biological. These stimuli include infectious agents, damaged cells, toxic substances, or irradiation. Inflammation is the process involved in removing such injurious stimuli by initiating the healing process [1]. The primary function of the inflammatory response is to identify and destroy the danger stimuli, repair or replace the injured tissue and reestablish tissue homeostasis. Inflammation can affect all organs of the body. The four cardinal signs of inflammation are redness (rubor), heat (calor), pain (dolor), swelling (tumor) and loss of function [2]. The inflammatory response includes a complex network of several cell types and is explained in the following steps:

1. At the site of injury, the epithelial and endothelial cells of the damaged tissue release inflammatory factors such as cytokines and chemokines, which are chemical substances that are rapidly synthesized and released in response to infection.

2. These inflammatory factors then induce vasodilation (widening of blood vessels leading to redness and heat), subsequently helping in extravasation of neutrophils (professional killer cells) and leakage of plasma (swelling) into the damaged tissue.

3. Followed by neutrophils, monocytes, leukocytes (white blood cells) and mast cells are deployed to damaged tissues via chemotaxis, a process that involves migration of cells to site of inflammation. Monocytes can further differentiate into macrophages.

4. Neutrophils and macrophages kill the damaging stimuli by a process called phagocytosis (cell eating), which involves internalizing and degrading of the infectious agent and subsequently clearing the debris.

5. The infectious agent is thus eliminated and is followed by tissue repair. This step majorly involves the synthesis of a fibrous extracellular matrix (ECM) to replace the injured tissue. ECM is the non-cellular compartment that lines the tissues to provide structural support to the tissues. Cells such as fibroblasts, macrophages and keratinocytes participate in this healing process. Inflammatory response thus is normally rapid, and it should be noted that prolonged inflammation can lead to tissue damage [3-5]. The main steps involved in the inflammatory response are illustrated in Figure 1. 


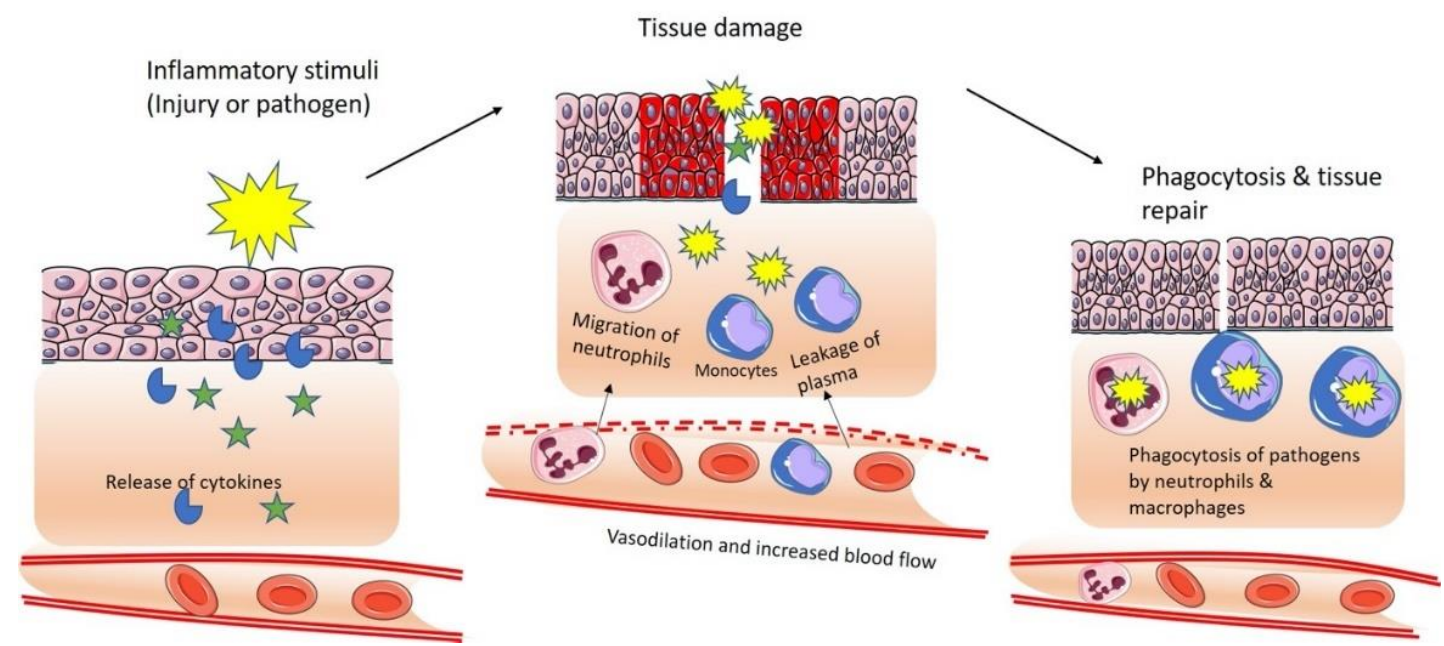

Figure 1 Major events in the local inflammatory response. Upon an inflammatory stimulus, cells release chemokines and cytokines that help in the vasodilation of blood vessels. This leads to recruitment/ infiltration of leucocytes (neutrophils and macrophages) which engulf the pathogen and initiate tissue repair. Figure is created with permission from Servier Medical Art Image bank.

As mentioned above, macrophages due to their phagocytic ability represent an integral part of the inflammatory processes and are critical regulators of both normal homeostasis as well as pathology. Based on the site of the origin, macrophages are classified either as blood monocyte-derived or tissue-resident macrophages. Macrophages are seeded throughout the body in various organs including liver, bone, lungs and intestine. During inflammation, macrophages mediate three major functions: antigen (foreign body) presentation, phagocytosis, and production of various cytokines and growth factors [6]. Further, macrophages themselves are activated by cytokines including interferon gamma, tumor necrosis factor alpha (TNF $\alpha$ ), extracellular matrix proteins and other chemical mediators. Macrophages also respond to metabolic cues such as glucose and lipids [7]. Thus, macrophages play and important role in metabolic and immune signaling in the body.

Based on the duration of the inflammatory response, inflammation can be either acute or chronic. Acute inflammation is the early response of the body to an inflammatory stimulus and is terminated after resolution of the inflammatory process. Therefore, acute inflammation typically lasts only for minutes to days and is mainly carried out by neutrophils. Failure to resolve inflammation or prolonged exposure to the triggering agent makes acute inflammation to become chronic. Chronic inflammation has a longer time course that can last from days up to years and is mediated by lymphocytes and macrophages rather than neutrophils). Moreover, acute inflammation is critical to maintain tissue homeostasis while chronic inflammation results in tissue damage leading to pathologies [8-10]. Major differences between acute and chronic inflammation are mentioned in Table 1. 
Table 1: Differences between acute and chronic inflammation

\begin{tabular}{|c|c|c|}
\hline Feature & Acute inflammation & Chronic inflammation \\
\hline Trigger & Injured tissue, pathogens & Persistent pathogens, metabolic disturbances \\
\hline Duration & Short (days to weeks) & Long (months to years) \\
\hline Cell types & Neutrophils, eosinophils, mast cells & Macrophages, lymphocytes \\
\hline Location & Local & Systemic \\
\hline Outcome & $\begin{array}{l}\text { Abscess formation and resolution of } \\
\text { inflammation }\end{array}$ & Tissue damage and fibrosis \\
\hline
\end{tabular}

Chronic low-grade inflammation is a mild sustained inflammation with low- levels of inflammatory markers such as C-reactive protein, interleukin-6 (IL-6) and tumor necrosis factor (TNF $\alpha$ ). Low- grade chronic inflammation observed in metabolic disorders is termed as 'metabolic inflammation' or 'meta- inflammation. While pathogens such as bacteria or viruses trigger classical or infectious inflammation, metabolic disturbances induce meta-inflammation. Pathologies associated with excess diet such as obesity, type 2 diabetes and non- alcoholic fatty liver disease (NAFLD) show metainflammation [11-13]. The chronic state of inflammation in these diseases is mediated by macrophages (among other immune cells) located within the liver, muscle, adipose tissue and colon. Another chronic inflammatory disorder, inflammatory bowel disease (IBD), is also partly governed by metabolic disturbances where the intake of a diet is believed to elicit certain inflammatory responses. On the other hand, the gut lining is populated with a multitude of bacteria, viruses and fungi, which also have been suggested to contribute to the inflammatory response observed during IBD. Therefore, the type of inflammation in IBD is likely a combination of metabolic and infectious inflammation. For example, the intake of a high fat diet alters the intestinal microbiota composition and disturbs the intestinal barrier leading to inflammation. Further, because the liver exists in proximity to the gastrointestinal tract, also the liver is strongly affected by the alterations in the intestinal microbiota. In fact, NAFLD and atherosclerosis are associated with changes in gut microbiota profile. Thus, gut microbiota forms a common ground for underlying pathologies including IBD and NAFLD. Even though these diseases have different triggers and affect different organs and display distinctive symptoms, they are known to share some common immunological features in their pathology. While inflammation seems to be more stable in metabolic diseases, IBD patients experience severe flares of acute inflammation [14]. Within the cluster of inflammatory diseases, the contents of this thesis will particularly focus on NAFLD and IBD.

\section{Inflammation at the center of non-alcoholic fatty liver disease}

Nonalcoholic fatty liver disease (NAFLD) is characterized by hepatic steatosis (which is $>/ 5 \%$ fat accumulation in the liver) with the lack of secondary causes such as significant alcohol consumption and other chronic liver diseases such as viral hepatitis [15]. NAFLD encompasses a whole spectrum of liver diseases ranging from hepatic steatosis, referred to as non-alcoholic fatty liver (NAFL) to nonalcoholic steatohepatitis (NASH) which is NAFL along with inflammation with or without liver fibrosis (damage of liver tissue). NASH can lead to liver cirrhosis (scarring of liver tissue) including the development of a hepatocellular carcinoma (HCC or liver cancer) [16,17], emphasizing the driving role of inflammation in the progression of NAFLD. Although NAFL is a largely benign and reversible condition, an estimated $20-33 \%$ of individuals with NAFL show indication of NASH on histopathology. 
Further, it is known that people with NASH progress to fibrosis and cirrhosis at a yearly rate of 7$10 \%$. Liver-related death in patients with NASH-related cirrhosis is around 1.4-3\% [18].

In the United States alone, the annual economic burden of NAFLD has been estimated at $\$ 103$ billion [19], while in European countries (i.e., the United Kingdom, Germany, France and Italy) these costs account for $€ 35$ billion and thus, have a large economic burden on health care systems [20]. Globally, it is currently estimated that NAFLD affects about one-quarter of the general population, while NASH has been calculated at $2-5 \%$ of the general population [21]. Estes et al. predicted a $63 \%$ increase in the prevalence of NASH by 2030 accounting to 27 million patients [22]. The primary risk factors for NAFLD development are obesity, type 2 diabetes, and the metabolic syndrome including dyslipidemia [23]. Additionally, genetic, environmental and demographic factors are likely to have some influence on the advancement of fatty liver disease [24]. Metabolic inflammation in the liver is a key driver of NAFLD disease progression. For example, excess lipid accumulation inside the liver activates intrahepatic inflammatory pathways leading to activated immune cells and generation of cytokines that further contribute to liver inflammation. The relationship between lipids and inflammation in NASH will be further elaborated in the following sections.

The majority of the NAFL patients are asymptomatic except for general complaints like fatigue and irritation in the upper right quadrant of the abdomen, often hindering a faster diagnosis [25]. Currently, liver biopsy continues to be the gold standard for diagnosing NAFLD/NASH; however, because of the invasiveness and cost of the biopsy, its use is limited [26]. On the other hand, reliable noninvasive methods to detect NASH are few, if any. Commonly, plasma levels of liver enzymes, alanine aminotransferase (ALT) and aspartate aminotransferase (AST) are often used in clinical practice as a surrogate for inflammation but have poor predictive value [27,28]. In general, aminotransferases cannot distinguish patients from steatosis to NASH. Next to the use of plasma markers, many imaging techniques such as ultrasound [29], computed tomography (CT) and magnetic resonance imaging (MRI) and FibroScan are increasingly used to detect different stages of NAFLD [30], but with limited assessment of inflammatory levels [31]. Therefore, future advances in the field of NASH diagnostics focusing on developing sensitive and specific non-invasive markers of inflammation are required.

\section{Inflammation as driving factor in Inflammatory Bowel Disease}

Inflammatory bowel disease (IBD) is a chronic inflammatory disorder of the gastrointestinal (GI) tract. The major types of IBD are Crohn's disease (CD) and ulcerative colitis (UC) which are associated with chronic intestinal inflammation and alterations in gut microbiota composition [32]. While UC is a condition in which the inflammatory responses are confined to colon, $\mathrm{CD}$ can involve any part of the $\mathrm{Gl}$ tract. The gut microbiome is defined as the totality of microorganisms including bacteria, viruses, protozoa, and fungi present in the gastrointestinal tract that help in executing several physiological functions of the body [33]. Individuals between the age group of 15-35 are more often diagnosed with IBD. The signs of IBD include stomach pain, blood and mucus in the stools, diarrhea and a poor quality of life [34]. The prevalence of IBD is highest in the Western countries affecting up to $0.5 \%$ of the general population. Overall, Europe has a higher prevalence of UC than CD while the contrary is seen in Australia; UC and CD are equally distributed in North America [35]. 
The etiology of IBD is largely not known. However, genetic, environmental and individual's factors including immune status and intestinal microbiota composition are shown to be implicated in the pathogenesis of IBD. Risk factors for IBD include smoking, hygiene, drugs, diet and stress. Moreover, it is also known that obesity might contribute to the development of IBD [36]. Importantly, the combination of genetic factors and environmental factors is known to impair intestinal barrier function leading to translocation of beneficial gut microorganisms into the bowel wall. Damage to the intestinal barrier is critical as it may allow increased access and assimilation of toxic and immunogenic factors. As a result of dysbiosis and intestinal barrier breach, the microbiota and its components (that might act as endotoxins) are leaked into the circulation and subsequently trigger inflammation. The inflammation cascade further triggers the inflow of neutrophils and macrophages that increase the pro-inflammatory cytokine production, subsequently leading to perpetuation of epithelial cell damage, gut inflammation and ulceration [37].

Similar to NAFLD, the pathogenesis of IBD is also at least partly driven by the contribution macrophages. Majority of the tissue-resident macrophages are formed before birth from embryonic macrophages or fetal liver cells and sustain themselves through limited self-renewal. In contrast, gutresident macrophages are the most abundant and are derived from lymphocyte antigen (Ly6C+) blood monocytes, cells which are associated with the inflammatory phenotype and are constantly renewed from hematopoietic stem cells (HSCs) [38]. The failure of Ly6C monocytes to differentiate into mature intestinal macrophages leads to their accumulation which causes inflammation in the intestine. This step subsequently results in excessive secretion of IL-23 and TNF $\alpha$. CD is catheterized by enhanced production of interferon-g and TNF $\alpha$. In ulcerative colitis, the local immune response is characterized by increased Interleukin- 13 levels [39].

\section{Pathophysiology of inflammation}

\section{Hub of lipid metabolism: The liver}

The liver is one of the largest organs in the body driving many important metabolic functions. The adult human liver weighs about 1.4 kilograms and is found in the right upper abdomen, below the diaphragm. Beneath the liver, the gallbladder is located. The gallbladder stores bile, which facilitates the digestion and absorption of fats (among other functions). The liver is a unique organ due to its dual blood supply: nutrient-rich blood from the portal vein (approximately 75\%) that drains the stomach, intestines, pancreas and spleen and the oxygen-rich arterial blood from the hepatic artery (approximately 25\%). The portal vein carries the blood from the digestive organs to the liver, carrying toxic substances, drugs and nutrients. The liver processes these substances with the help of its enzymes such as cytochromes. Once degraded, modified or detoxified they are sent back into the blood or excreted. Major cell types found in the liver are hepatocytes, endothelial cells, Kupffer cells (liver resident macrophages) and stellate cells (hepatic development and regeneration). Hepatocytes majorly participate in metabolic functions; endothelial cells play a role in immunity by scavenging substances, while Kupffer cells mediate inflammatory responses [40].

Liver is the main organ involved in body's energy metabolism. This energy is primarily derived from carbohydrates, sugars and fats from the food we consume. After a meal, dietary sugars and fats are digested in different organs of the body to release energy. Lipids are fat-like substances that are 
absorbed from food or synthesized in the liver and are critical for cell structure, function, and energy, as well as for insulation and protection. Important type of lipids are triglycerides and cholesterol [41]. Due to their hydrophobic nature (insoluble in water), lipids are packaged and transported through the bloodstream in the form of lipoproteins. Based on their content (lipid and protein combination), density and size, lipoproteins are classified as chylomicrons, very low-density lipoproteins (VLDL), low-density lipoproteins (LDL) and high-density lipoproteins (HDL). Lipid metabolism and transport is organized into three transport systems: (a) exogenous transport that is associated with dietary lipids (b) endogenous transport which is associated with internally produced lipids and (c) reverse transport which transports the lipids from the periphery (e.g., skeletal muscle and adipose tissue) to the liver.

The exogenous pathway begins with the intestinal absorption of triglycerides and cholesterol from dietary sources. After absorption, these dietary lipids are coupled with various proteins to form chylomicron particles. Chylomicrons are hydrolyzed by lipoprotein lipase (LPL) expressed by endothelial cells into free fatty acids and glycerol that are ultimately taken up by the cell via cell surface receptors like cluster of differentiation 36 (CD36). The muscle, white and brown adipose tissue are major recipients of such triglyceride cargo. The remnants of chylomicrons are endocytosed by hepatocytes by receptors on the liver such as the low-density lipoprotein (LDL) receptor (LDLr), LDL receptor- related protein (LRP). An overview on lipoprotein metabolism is depicted in Figure 2.

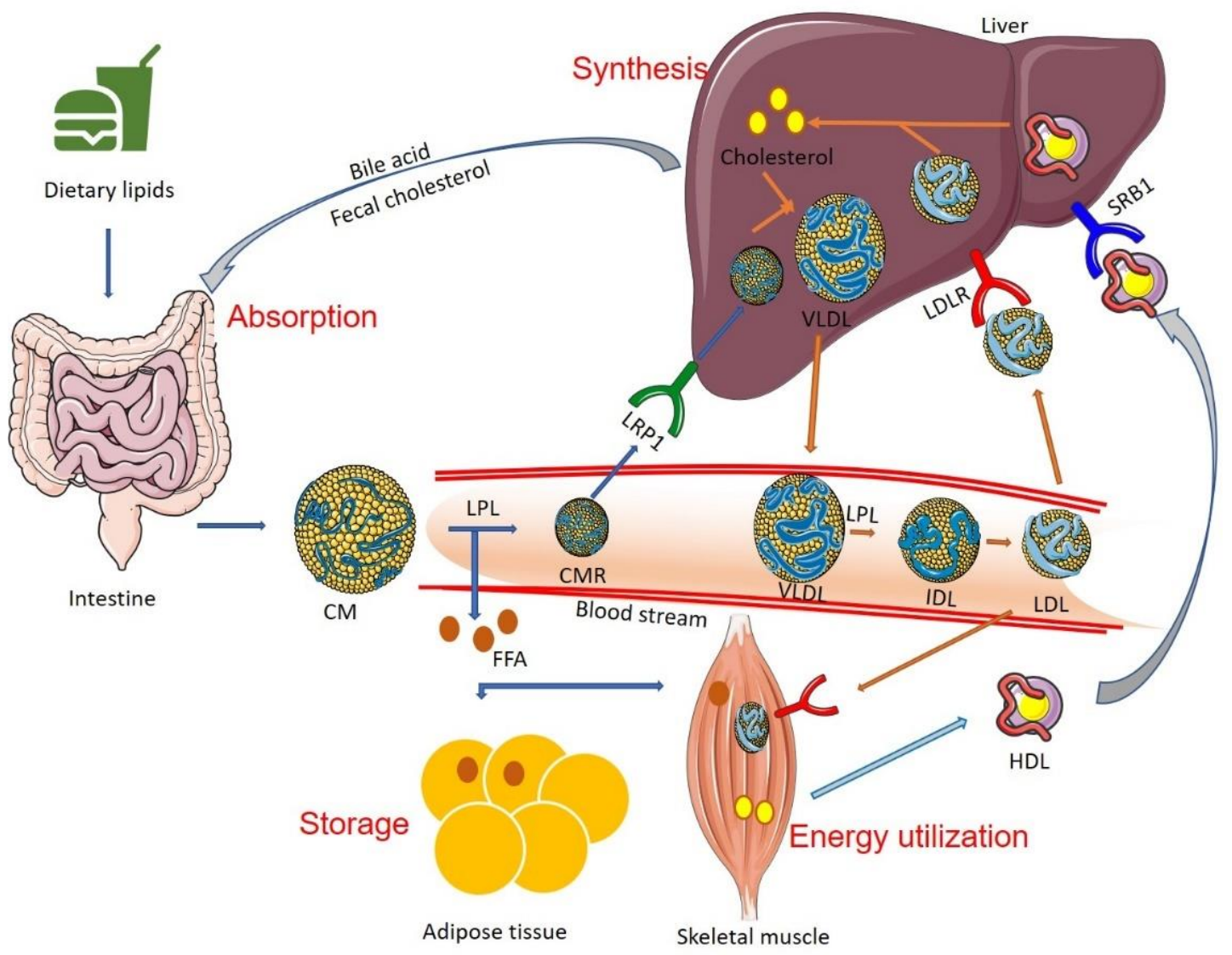

Figure 2 Lipoprotein metabolism. CM: chylomicrons; LPL: lipoprotein lipase; FFA: free fatty acids; CMR: chylomicron remnants; LRP1: LDL-related receptor 1; TG: triglyceride; C: cholesterol; VLDL: verylow-density lipoprotein; IDL: intermediate-density lipoprotein; LDL: low-density lipoprotein; LDLR: low-density lipoprotein receptor; HDL: high-density lipoprotein; SRB1: scavenger receptor class B type I. Figure is created with permission from Servier Medical Art Image bank. 
When the supply of dietary lipids is low, chylomicron remnants are combined with newly synthesized lipids in the liver to form VLDL particles which initiate the endogenous pathway. Similar to chylomicrons, VLDL are hydrolyzed by LPL by detachment of the remaining triglycerides and its substitution by cholesterol esters to form intermediate-density lipoproteins (IDLs) and LDLs. Majority of these LDL particles are cleared by liver. After the uptake of LDL particles by hepatic macrophages, they are directed to lysosomes for subsequent degradation. Lysosomes are 'recycling centers' of the cell and are characterized by an acidic interior, which enables the activity of many hydrolytic enzymes including proteases, lipases, and nucleases $[42,43]$. Under normal conditions, cholesterol is hydrolyzed by the lysosomal acid lipase (LAL) in the lysosomes and the free unesterified cholesterol that is formed is transferred to the cytoplasm with the help of Niemann-pick disease type C1 (NPC1) and NPC2 proteins. While NPC2 plays an obligate role in shuttling cholesterol through the lysosomal lumen, NPC1, which is located on the lysosomal membrane is known to efflux the free cholesterol into the cytoplasm [44]. Some of the LDL particles absorbed by the extrahepatic peripheral tissue such as skeletal muscle.

In reverse cholesterol transport, cholesterol is transferred from peripheral tissues back to the liver for ultimate excretion with the help of HDLs. This is important for homeostasis as most cells in peripheral organs cannot catabolize cholesterol. HDL particles are synthesized as cholesterol-free particles by intestinal enterocytes and liver. Acquiring cholesterol generates mature HDL particles which then return to the liver via scavenger receptor class B type 1 (SR-B1) receptors on macrophages. Cholesterol is hydrolyzed and excreted into bile acid. Finally, the cholesterol depleted HDLs are either enter the circulation to be reused or excreted [45].

\section{Excess lipid accumulation in the macrophages induces lysosomal dysfunction and inflammation}

Lipid homeostasis in the cells is maintained by balancing lipid synthesis, transport and metabolism. Any imbalance to the normal process including lipid excess that is commonly observed in obesity, disrupts lipid homeostasis [46]. Especially, cholesterol accumulation leads to a shift in the lipoprotein profile towards increased LDL levels and reduced HDL levels, a condition referred to as dyslipidemia. Also, metabolic disorders such as NAFLD, atherosclerosis and insulin resistance are usually associated with dyslipidemia $[47,48]$.

Due to elevated LDL levels during dyslipidemia, cholesterol metabolism in macrophages is overwhelmed leading to excessive accumulation of LDL. Moreover, cholesterol overload is associated with abnormalities such as oxidative stress [49]. Oxidative stress is defined as a process caused by an imbalance between production and detoxification of reactive oxygen species (ROS) in cells and tissues. Due to increased presence of oxidative stress in dyslipidemic conditions, LDL particles are highly prone to oxidation, which increases the levels of oxidized LDL (oxLDL) particles [50,51]. Unlike $L D L$, which is endocytosed by $L D L$ receptors, oxLDL is taken up by scavenger receptors present on the macrophages. The two main scavenger receptors responsible for oxLDL uptake are scavenger receptor A (SR-A) and CD36 [52]. Since the structure of oxLDL particles mimics the epitopes of pathogens, oxLDL is taken up by scavenger receptors as a part of protective response. However, this might cause oxLDL overload in the macrophages [53]. Moreover, normal LDL is efficiently cleared from lysosomes as described earlier, but oxLDL is resistant to normal degradation and efflux and therefore accumulates in the lysosomal compartments [54]. This chain of events is known to trigger 
inflammatory pathways during atherosclerosis and NASH [55-58]. Thus, inflammation and metabolism are capable of regulating one another.

One of the potential mechanisms that explains the link between oxLDL and hepatic inflammation is via lysosomal membrane permeabilization. Excessive oxLDL inside the lysosomes can damage and disturb lysosomal membrane leading to the release of the lysosomal components incusing its enzymes into cytoplasm and subsequently into extracellular space, a process called as lysosomal exocytosis $[59,60]$. The intracellular cholesterol metabolism in normal and dyslipidemic conditions is illustrated in Figure 3.
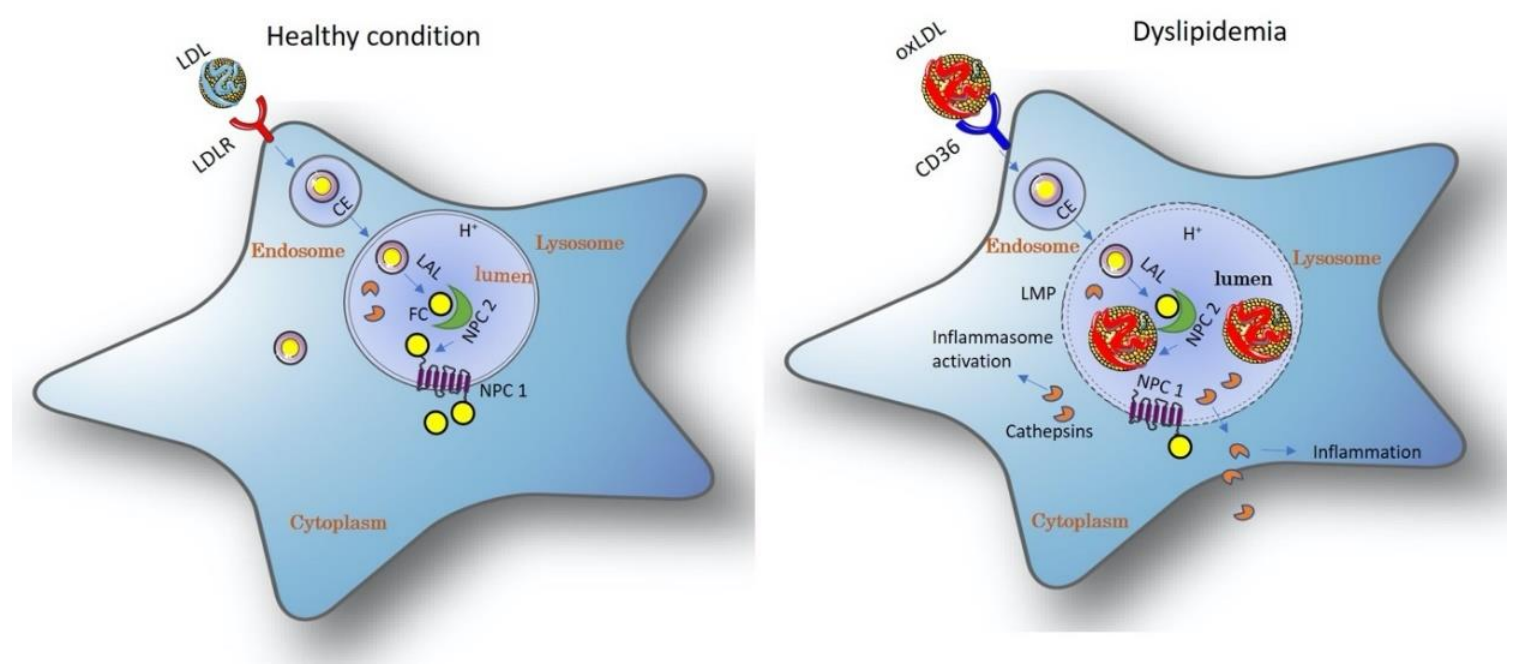

Figure 3 Intracellular cholesterol metabolism. LDL is taken up by the cells via LDLR. Cholesteryl esters (CEs) from the endosome are directed towards the lysosome, where they are hydrolyzed by lysosomal acid lipase ( $L A L)$, generating free unesterified cholesterol (FC). FC is transported to the lysosomal lumen by NPC2 and to cytoplasm via NPC1 proteins. During dyslipidemia, LDL gets modified to oxLDL and is taken up by the cells via CD36 receptor. Rather than being transported to cytoplasm, oxLDL is resistant to efflux and accumulates in the lysosomes. This leads to rupture of lysosomes by lysosomal membrane permeabilization (LMP) causing leakage of lysosomal enzymes into cytoplasm and into the extracellular space. These changes of events trigger inflammatory responses. LDL, low-density lipoprotein; CE, cholesteryl ester; FC, free unesterified cholesterol; LAL, lysosomal acid lipase; NPC, Niemann-Pick type C; LMP- lysosomal membrane permeabilization. Figure is created with permission from Servier Medical Art Image bank.

Intralysosomal oxLDL can additionally contribute to activation of inflammation by lysosomal enzymes. Lysosomes harbor several kinds of enzymes including lipases, nucleases, proteases which work together in the degradation of various substances. One of the most abundant lysosomal enzymes are cathepsins, which are proteases that help in intracellular protein degradation in the lysosomes [61]. Cathepsins are normally located in the acidic environment of lysosomes, however in some physiological and pathological conditions they can be secreted out the lysosomes into the cytosol or outside of the cell. Cytosolic cathepsins are known to involved in mitochondrial dysfunction, apoptosis (cell death) [62] and inflammasome activation [63]. Inflammasomes are multiprotein complexes that are activated upon bacterial infections or cellular damage. Cathepsins activate/degrade components of inflammasome and thereby trigger the release of the proinflammatory cytokines leading to inflammation. Moreover, lysosomal dysfunction impairs 
autophagy, a self-destructive process that mediates intracellular degradation of substances. Impaired autophagy enhances ROS production, inflammasome activation, and IL-1ß production. Disturbed autophagy is reported in NASH and IBD [64]. Alternatively, cathepsins in the extracellular space are known to participate extensively in the degradation of matrix substances leading to inflammation [65]. All these findings show an association between cholesterol-loaded macrophages, lysosomal exocytosis, cathepsins and inflammation during dyslipidemia. Interestingly, we have previously shown that plasma cathepsin D is implicated in NASH patients [66].

\section{Role of cathepsin D in inflammation}

Cathepsin D is an aspartic protease that is constitutively expressed in nearly all cells and is located typically in the lysosomes where it participates in protein degradation. Loss of this proteolytic activity results in accumulation of substances in different cell types leading to neurodegeneration, developmental and vision related problems. Active and zymogen (inactive) forms of cathepsin $D$ are known to be released into cytosol and into circulation by macrophages, mammary epithelial cells, cancer cells and keratinocytes. Cathepsin D in its active form was found in biological fluids such sweat, urine, seminal fluid and also in macrophage- conditioned media [67]. The lysosomal fraction of cathepsin D has more 'house-keeping' functions while the extracellular fraction is most likely associated with pathological conditions. For example, increased levels and activity of cathepsin D in extracellular space are reported in several metabolic inflammatory disorders including atherosclerosis [68], diabetes [69], cancer [70] and IBD [71]. Moreover, it can regulate inflammation by many ways. For example, proteolytic alterations of apolipoprotein B-100 (apoB) part of LDL by cathepsin D causes LDL accumulation in arterial intima, a main feature of atherosclerosis. In IBD, cathepsin $\mathrm{D}$ contributes to inflammation by the destruction of extracellular matrix and basement membrane proteins that can lead to impairment of the intestinal barrier and epithelial cell damage. Furthermore, we have previously observed that plasma cathepsin D correlated with hepatic inflammation in NAFLD/ NASH patients [66] and demonstrated the functional link of cathepsin D in $\mathrm{NASH}$-related hepatic inflammation [72].

\section{Role of stress in inflammation}

One of the potential risk factors for the development of inflammatory disorders is stress [73]. Stress is defined as the outcome of any internal or external stimuli that disrupts the body's homeostasis. The concept of stress has two components: 1 ) stressors (the triggers or factors that cause stress) and 2) stress (the bodily reaction to stressors). Stress can be physical (trauma, illness and dietary) or psychological (fear, anxiety, depression etc.). Humans are constantly subjected to variety of stressors from pre-natal life to adulthood that can largely influence the health status of the individual. Evidence suggests that exposure to stressors such as smoke, diet, medication and psychosocial stress during early life and childhood is known to have adverse effects in adult life. Socio-economic status, work conditions and trauma majorly trigger stress in adult life [74].

Stress is linked to many diseases including diabetes, cardiovascular diseases, obesity and IBD. Stressful conditions are known to trigger unhealthy eating habits [75]. Moreover, stress is known to disrupt lipid metabolism and increase postprandial levels of triglycerides [76]. Not just disrupting metabolic activities, stress is often accompanied by elevated inflammatory responses. Severe stress 
leads to hyperactive immune system leading to disturbed inflammatory responses. In fact, chronic low-grade inflammation is considered as a link between stress and the underlying pathophysiology of metabolic diseases like diabetes. Evidence from preclinical and clinical studies demonstrated stress stimuli to the brain activates autonomic nervous system and hypothalamic-pituitary-adrenal axis (HPA axis) which increase pro-inflammatory cytokine production by modulating several pathways [77]. For example, in IBD, stress induced changes in the brain are known to affect the gut microbiota composition, intestinal permeability and cytokine secretion, thereby increasing the severity of the disease. However, the complex mechanisms responsible for the stress-induced increase of plasma inflammatory markers in inflammatory diseases are beyond the scope of this thesis.

\section{Resolving inflammation}

\section{Lifestyle modifications}

Lifestyle modifications are the first-line approach to manage chronic inflammatory diseases [78]. Healthy lifestyle which includes calorie restriction, healthy food choices, physical activity and stress management are proven to reduce levels of proinflammatory markers such as CRP [79], delay or prevent the onset of type 2 diabetes [80] and NASH [81].

Diet: Obesity is usually associated with adipose tissue hypoxia that results in triggering of the NF-kBinducible signaling leading to production of proinflammatory cytokines. Although the mechanism is unclear, foods rich in fibers, whole grains, omega- 3 fatty acids and antioxidant vitamins are known to reduce blood concentration of inflammatory markers such as CRP and TNF $\alpha$ [82]. Though the firstline therapy for NASH is calorie restriction, majority of NASH patients are unable to attain and retain the required amount of weight loss [83]. For morbidly obese individuals who are unable to reduce weight, bariatric surgery is considered. Bariatric surgery introduces changes in the digestive system restricting the amount of food that enters the stomach. Though bariatric surgery is known to improve physical health and ameliorate NASH histology, it is known to have complications and relapse of NASH after surgery [84].

Physical activity: Physical exercise is one of the important intervention strategies that is widely prescribed for lowering the risk of chronic diseases and is known to have modulatory effect on inflammation. Literature suggests that moderate physical activity in young and elderly population as well as in metabolic syndrome patients led to beneficial anti-inflammatory effects [85]. Although the underlying mechanism is not completely known, exercise is known increase adipose tissue blow flow and thus blunting the hypoxia-associated inflammation. Besides the reduction of inflammatory markers, exercise has been documented to restore liver enzyme levels and improve the histological alterations associated with metabolic syndrome [86].

Relaxation: Similar to diet and physical activity, various relaxation methods to reduce stress are known to have beneficial effects in patients. Effective stress management training is known to reduce inflammatory markers in metabolic diseases and IBD [87]. Stress management includes selfrelaxation techniques such as yoga, meditation, mind relaxation and guided therapy (amongst others) which mainly help in reducing heart rate, anxiety and improving quality of life in general [88]. The role of stress-management strategies in tackling inflammation are discussed in this thesis. 
Despite strong evidence for the benefits of lifestyle modification, achieving and maintaining these changes remains a challenge. Major concerns include the compliance of the patients to lifestyle modifications due to economic problems, physical disabilities, or the difficulty to present a personalized plan for patients. Moreover, each patient might require different dietary recommendations, duration and/frequency of food intake. These factors will influence participant's adherence and their success in achieving the best results. Unfortunately, most health professionals currently lack training and means to manage lifestyle interventions for their patients [89]. Lack of data on prolonged effects of lifestyle medicine and heterogeneity in the observed effects between patient groups also makes it difficult to formulate lifestyle plans. Additionally, stress interventions didn't prove to be consistently effective in producing effects in work settings [90]. Failure to adhere to stress-management techniques and constantly emerging stress situations in the workplaces signal that stress will increase or at least continue to be a problem in the near future. For all of these reasons, lifestyle interventions are often supported with pharmacological tools.

\section{Pharmacological targets in inflammatory disorders}

To support the dietary and lifestyle interventions, pharmacological agents to reduce inflammation are widely used in treating metabolic diseases. Pharmacological therapies for NASH are being extensively researched, although none of them can convincingly reverse NASH yet. The NASH drug market is set to increase from $\$ 618$ million in 2016 to approximately $\$ 25.3$ billion by 2026, pointing towards the huge demand for NASH treatments [91]. Guidelines from the American Association for the Study of Liver Diseases advise pioglitazone as a treatment for $\mathrm{NASH}$, but this drug is shown to have increased risk of prostate or pancreatic cancer, body weight gain and fluid retention [92]. Four drug candidates for NASH are currently in phase 3 clinical trials namely, obeticholic acid, elafibranor, cenicriviroc, and resmetirom. These candidates are yet to receive approval from the food and drug administration (FDA) to be used as prescription medications [91]. Therefore, currently the only curative therapy for NASH is liver transplantation, which involves replacing the patient's liver with a healthy donor liver accompanied by persistent lifestyle changes. Scarcity of the donor organs and post-surgery complications urges the need to develop alternate therapeutic tools [93]. Therefore, research dedicated at identifying the complex molecular pathways triggering inflammation is highly desirable to formulate effective treatment options for NASH.

Management of IBD relies on a range of pharmacological and surgical interventions based on age [94]. Aminosalicylates are often the first option in the treatment of IBD to reduce inflammation but are limited by their efficacy [95]. Corticosteroids are used as the second option for the management of moderate-to-severe IBD. Though they offer rapid relief of symptoms and reduce inflammation, side effects including bone damage and risk of infection are observed in patients [96]. Immunosuppressants that control the hyperactive immune system are although extremely useful for IBD patients, they cannot offer complete treatment. Normally used immunosuppressant drugs include azathioprine, methotrexate, monoclonal antibodies against inflammatory cytokines, TNF, IL12 or IL-23, and $\alpha 4 \beta 7$ integrin [97]. In elderly, surgery to remove the colon is considered curative for UC, but it is not a treatment of choice for most patients [98].

Alternative therapies include agents such as statins because of their anti-inflammatory as well as cholesterol modulating properties. Statins are known to reduce LDL concentrations up to $30 \%$. Statins reduce cholesterol by inhibiting HMG-coA (3-hydroxy-3-methyl-glutaryl-CoA) reductase, the 
main enzyme involved in cholesterol synthesis in liver. Statins are known to modulate inflammation by reduction of pro-inflammatory cytokine production and by inhibition of T-cell activation and proliferation at the site of infection. Side effects of statin treatment include joint and muscle pains and potential interaction of statins with other medications [99]. One of the available statin alternatives is 2-hydroxypropyl- $\beta$-cyclodextrin (CD). CD is an FDA-approved compound that is normally used for delivering therapeutic drugs because of its ability to solubilize lipophilic substances. Due to its cholesterol solubilizing properties, CD is a well-established treatment option for NiemannPick disease type C1 disease (NPC1), a lysosomal storage disorder. Moreover, in mouse models of atherosclerosis, cyclodextrin showed beneficial effects as it converted the cholesterol crystals into water-soluble 27-hydroxycholesterol, promoted cholesterol efflux and exerted anti-inflammatory effects [100]. However, CDs are shown to have to toxic effects on different organs including ear (hearing loss) and kidney. Moreover, long term administration of CD is known to cause bone loss [101]. Hence novel therapeutic targets that modulate inflammation with minimal or zero side effects are essential. To this end, targeted drug approaches that involves selective inhibition of targets are considered safer and novel [102]. In this regard, small molecules are being investigated as standalone drugs because of their ability to reach precise target areas. Besides, they are well tolerated and easy to synthesize and optimize [103]. This thesis focuses on understanding the underlying mechanism of inflammation in metabolic diseases and optimization of pharmacological targets in pre-clinical rodent models.

\section{Pre-clinical models for validation of putative targets against NAFLD}

To study the mechanistic details of inflammatory processes, we made use of rodent models in this thesis. Preclinical animal models are critical for not only studying the disease progression, but also to test the potential of therapeutic drugs. Since excess dietary intake is one of the main causal factors for NAFLD in humans, dietary animal models are usually preferred to perform NAFLD/NASH research. Many dietary rodent models have tried to mirror the metabolic disturbances as well as histological features observed in human NAFLD and NASH [104]. In rodents, NASH is usually induced by feeding high-carbohydrate or high-fat diets (HFDs), methionine and choline-deficient (MCD) diet, cholinedeficient L-amino acid-defined (CDAA) diet and others.

\section{Hyperlipidemic LdIr-/- as a mouse model for NASH}

One of the existing mouse models that mimics most features of human NASH is the low-density lipoprotein receptor Ldlr-/- mouse model. This is the combinatorial model based on the genetic deletion of the low-density lipoprotein receptor (Ldlr) with HFD for a period of 3-12 weeks. Due to the absence of Ldlr, these mice lack the ability to uptake LDL into the cells and thus display high levels of plasma LDL and low levels of HDL, hence resembling human-like lipoprotein profile. Moreover, Ldlr-/- mice show sustained hepatic inflammation. While these mice do not develop severe fibrosis, they show mild to moderate fibrosis compared to normal C57BL/6 mice on the same diet. Therefore, Ldlr-/- model serves as an excellent pre-clinical model for early inflammation studies associated with human NASH $[105,106]$. 


\section{Chapter 1}

\section{Sprague-Dawley rats as model for hepatic steatosis}

Similar to mice, rat models differing in nutritional and genetic factors are frequently used as preclinical models for NAFLD/NASH. In fact, compared to mouse models, rat models are more susceptible to HFD, and are shown to display severe early histological features of NAFLD [107]. When given a HFD ( $71 \%$ fat $/ 11 \%$ carbohydrates/ $18 \%$ proteins) for a period of 3 weeks, Sprague-Dawley rats were reported to develop steatosis, insulin resistance and mild to marked inflammation, features also observed in human NASH [108]. 


\section{Thesis aim and outline}

Low-grade systemic inflammation plays a crucial role in the development of NAFLD and IBD. Current treatment of chronic inflammatory diseases relies mainly on lifestyle changes and lowering lipid or inflammatory mediators. However, there are no existing approved FDA treatments. Good understanding of the pathophysiological processes involved in these diseases can help to formulate safe and effective therapies. In the current thesis, we aimed to characterize the mechanisms linking lysosomal lipid overload and inflammation investigated the effects of different intervention strategies to treat metabolic inflammation.

Chapter 2 provides extensive information about the role of cathepsins in pathophysiological conditions. Further, we specifically explain the mechanisms of cathepsin translocation and address the site-specific functions of cathepsins with emphasis on the importance of selective cathepsin targeting in chronic inflammatory diseases. Chapter 3 explores the pharmacological value of a selectively targeting extracellular cathepsin D in NAFLD using a small-molecule inhibitor. This chapter demonstrates the causal role of extracellular cathepsin D in lipid metabolism using Sprague-Dawley rats on high fat diet. In addition to steatosis, we found improved insulin sensitivity in these rats. Since steatotic rats display different hyperlipidemic profile than humans, we further expanded this research in chapter 4, where we investigated the individual roles of intracellular and extracellular cathepsin fractions in hepatic inflammation. We made use of $L d l r-/$ - mice which were treated with respective cathepsin inhibitors and fed a high- fat, high-cholesterol diet for a period of 10 weeks. Furthermore, we used proteomic approaches to understand the mechanisms of intra and extracellular cathepsin D action during NASH. In chapter 5, we explain the complex role of the gut/microbiota/brain axis with regard to the gastrointestinal inflammation associated with IBD. Further this chapter focuses on the benefits of non-pharmacological stress management techniques in modulating inflammation in IBD. In chapter 6 , the effects of 2 -hydroxypropyl- $\beta$-cyclodextrin (CD), a cholesterol mobilizing drug were examined in various models of metabolic inflammation Using in vivo and a series of in vitro experiments, we demonstrated that besides its beneficial cholesterol depleting properties, CD can cause time-dependent pro-inflammatory effects. Thus, care should be taken when using $C D$ in clinic. Finally, chapter $\mathbf{7}$ discusses the important conclusions of this thesis with respect to the current knowledge in the field and highlights the clinical implications of the findings. 


\section{References}

1. Chen, L., et al., Inflammatory responses and inflammation-associated diseases in organs. Oncotarget, 2018. 9(6): p. 7204-7218.

2. Hakansson, A. and G. Molin, Gut microbiota and inflammation. Nutrients, 2011. 3(6): p. 637-82.

3. Medzhitov, R., Inflammation 2010: new adventures of an old flame. Cell, 2010. 140(6): p. 771-6.

4. Midwood, K.S., L.V. Williams, and J.E. Schwarzbauer, Tissue repair and the dynamics of the extracellular matrix. Int J Biochem Cell Biol, 2004. 36(6): p. 1031-7.

5. Serhan, C.N. and J. Savill, Resolution of inflammation: the beginning programs the end. Nat Immunol, 2005. 6(12): $\mathrm{p}$. 1191-7.

6. Netea, M.G., et al., A guiding map for inflammation. Nat Immunol, 2017. 18(8): p. 826-831.

7. Fujiwara, N. and K. Kobayashi, Macrophages in inflammation. Curr Drug Targets Inflamm Allergy, 2005. 4(3): p. 2816.

8. Doggrell, S.A., Inflammation, the key to much pathology. Drug News Perspect, 2005. 18(8): p. 531-9.

9. Kacirova, M., et al., Inflammation: major denominator of obesity, Type 2 diabetes and Alzheimer's disease-like pathology? Clin Sci (Lond), 2020. 134(5): p. 547-570.

10. Stone, W.L., H. Basit, and B. Burns, Pathology, Inflammation, in StatPearls. 2020: Treasure Island (FL).

11. Esser, N., et al., Inflammation as a link between obesity, metabolic syndrome and type 2 diabetes. Diabetes Res Clin Pract, 2014. 105(2): p. 141-50.

12. Hotamisligil, G.S., Inflammation and metabolic disorders. Nature, 2006. 444(7121): p. 860-7.

13. Xu, H., Obesity and metabolic inflammation. Drug Discov Today Dis Mech, 2013. 10(1-2).

14. Belizario, J.E., J. Faintuch, and M. Garay-Malpartida, Gut Microbiome Dysbiosis and Immunometabolism: New Frontiers for Treatment of Metabolic Diseases. Mediators Inflamm, 2018. 2018: p. 2037838.

15. Chalasani, N., et al., The diagnosis and management of nonalcoholic fatty liver disease: Practice guidance from the American Association for the Study of Liver Diseases. Hepatology, 2018. 67(1): p. 328-357.

16. Kleiner, D.E., et al., Design and validation of a histological scoring system for nonalcoholic fatty liver disease. Hepatology, 2005. 41(6): p. 1313-21.

17. Brown, G.T. and D.E. Kleiner, Histopathology of nonalcoholic fatty liver disease and nonalcoholic steatohepatitis. Metabolism, 2016. 65(8): p. 1080-6.

18. Satapathy, S.K. and A.J. Sanyal, Epidemiology and Natural History of Nonalcoholic Fatty Liver Disease. Semin Liver Dis, 2015. 35(3): p. 221-35.

19. Puri, P. and M. Fuchs, Population Management of Nonalcoholic Fatty Liver Disease. Fed Pract, 2019. 36(2): p. $72-82$.

20. Abdelmalek, M.F., NAFLD: The clinical and economic burden of NAFLD: time to turn the tide. Nat Rev Gastroenterol Hepatol, 2016. 13(12): p. 685-686.

21. Younossi, Z.M., et al., The economic and clinical burden of nonalcoholic fatty liver disease in the United States and Europe. Hepatology, 2016. 64(5): p. 1577-1586.

22. Estes, C., et al., Modeling the epidemic of nonalcoholic fatty liver disease demonstrates an exponential increase in burden of disease. Hepatology, 2018. 67(1): p. 123-133.

23. Angulo, P., Gl epidemiology: nonalcoholic fatty liver disease. Aliment Pharmacol Ther, 2007. 25(8): p. 883-9.

24. Kneeman, J.M., J. Misdraji, and K.E. Corey, Secondary causes of nonalcoholic fatty liver disease. Therap Adv Gastroenterol, 2012. 5(3): p. 199-207.

25. Rinella, M.E., Nonalcoholic fatty liver disease: a systematic review. JAMA, 2015. 313(22): p. 2263-73.

26. Vuppalanchi, R., et al., Effects of liver biopsy sample length and number of readings on sampling variability in nonalcoholic Fatty liver disease. Clin Gastroenterol Hepatol, 2009. 7(4): p. 481-6.

27. Younossi, Z.M., et al., Diagnostic modalities for nonalcoholic fatty liver disease, nonalcoholic steatohepatitis, and associated fibrosis. Hepatology, 2018. 68(1): p. 349-360.

28. Mofrad, P., et al., Clinical and histologic spectrum of nonalcoholic fatty liver disease associated with normal ALT values. Hepatology, 2003. 37(6): p. 1286-92.

29. Hamaguchi, M., et al., Identification of individuals with non-alcoholic fatty liver disease by the diagnostic criteria for the metabolic syndrome. World J Gastroenterol, 2012. 18(13): p. 1508-16.

30. Lee, S.S. and S.H. Park, Radiologic evaluation of nonalcoholic fatty liver disease. World J Gastroenterol, 2014. 20(23): p. 7392-402.

31. Li, Q., et al., Current status of imaging in nonalcoholic fatty liver disease. World J Hepatol, 2018. 10(8): p. 530-542.

32. Macfarlane, G.T., et al., The gut microbiota in inflammatory bowel disease. Curr Pharm Des, 2009. 15(13): p. 1528-36.

33. Zuo, T. and S.C. Ng, The Gut Microbiota in the Pathogenesis and Therapeutics of Inflammatory Bowel Disease. Front Microbiol, 2018. 9: p. 2247.

34. Seyedian, S.S., F. Nokhostin, and M.D. Malamir, A review of the diagnosis, prevention, and treatment methods of inflammatory bowel disease. J Med Life, 2019. 12(2): p. 113-122.

35. Kaplan, G.G., The global burden of IBD: from 2015 to 2025. Nat Rev Gastroenterol Hepatol, 2015. 12(12): p. 720-7.

36. Ananthakrishnan, A.N., Environmental risk factors for inflammatory bowel disease. Gastroenterol Hepatol (N Y), 2013. 9(6): p. 367-74. 
37. Neurath, M.F., Targeting immune cell circuits and trafficking in inflammatory bowel disease. Nat Immunol, 2019. 20(8): p. 970-979.

38. Kuhl, A.A., et al., Diversity of Intestinal Macrophages in Inflammatory Bowel Diseases. Front Immunol, 2015. 6: p. 613.

39. Monteleone, G., et al., New mediators of immunity and inflammation in inflammatory bowel disease. Curr Opin Gastroenterol, 2006. 22(4): p. 361-4.

40. Kalra, A., et al., Physiology, Liver, in StatPearls. 2020: Treasure Island (FL).

41. Rui, L., Energy metabolism in the liver. Compr Physiol, 2014. 4(1): p. 177-97.

42. Settembre, C. and A. Ballabio, Lysosome: regulator of lipid degradation pathways. Trends Cell Biol, 2014. 24(12): p. 743-50.

43. Appelqvist, H., et al., The lysosome: from waste bag to potential therapeutic target. J Mol Cell Biol, 2013. 5(4): p. 21426.

44. Frolov, A., et al., NPC1 and NPC2 regulate cellular cholesterol homeostasis through generation of low density lipoprotein cholesterol-derived oxysterols. J Biol Chem, 2003. 278(28): p. 25517-25.

45. Feingold, K.R. and C. Grunfeld, Introduction to Lipids and Lipoproteins, in Endotext, K.R. Feingold, et al., Editors. 2000: South Dartmouth (MA).

46. Hegele, R.A., Plasma lipoproteins: genetic influences and clinical implications. Nat Rev Genet, 2009. 10(2): p. 109-21.

47. Ginsberg, H.N., Y.L. Zhang, and A. Hernandez-Ono, Metabolic syndrome: focus on dyslipidemia. Obesity (Silver Spring), 2006. 14 Suppl 1: p. 41S-49S.

48. Blaton, V., How is the Metabolic Syndrome Related to the Dyslipidemia? EJIFCC, 2007. 18(1): p. 15-22.

49. Bhatti, J.S., G.K. Bhatti, and P.H. Reddy, Mitochondrial dysfunction and oxidative stress in metabolic disorders - A step towards mitochondria based therapeutic strategies. Biochim Biophys Acta Mol Basis Dis, 2017. 1863(5): p. 1066-1077.

50. Butler, D. and B.A. Bahr, Oxidative stress and lysosomes: CNS-related consequences and implications for lysosomal enhancement strategies and induction of autophagy. Antioxid Redox Signal, 2006. 8(1-2): p. 185-96.

51. Kurz, T., et al., Lysosomes and oxidative stress in aging and apoptosis. Biochim Biophys Acta, 2008. 1780(11): p. 1291303.

52. Kunjathoor, V.V., et al., Scavenger receptors class A-I/II and CD36 are the principal receptors responsible for the uptake of modified low density lipoprotein leading to lipid loading in macrophages. J Biol Chem, 2002. 277(51): p. 49982-8.

53. Itabe, H., T. Obama, and R. Kato, The Dynamics of Oxidized LDL during Atherogenesis. J Lipids, 2011. 2011: p. 418313.

54. Jerome, W.G., et al., Lysosomal lipid accumulation from oxidized low density lipoprotein is correlated with hypertrophy of the Golgi apparatus and trans-Golgi network. J Lipid Res, 1998. 39(7): p. 1362-71.

55. Gibson, M.S., N. Domingues, and O.V. Vieira, Lipid and Non-lipid Factors Affecting Macrophage Dysfunction and Inflammation in Atherosclerosis. Front Physiol, 2018. 9: p. 654.

56. Bieghs, V., et al., Internalization of modified lipids by CD36 and SR-A leads to hepatic inflammation and lysosomal cholesterol storage in Kupffer cells. PLoS One, 2012. 7(3): p. e34378.

57. Bieghs, V., et al., Role of scavenger receptor $A$ and CD36 in diet-induced nonalcoholic steatohepatitis in hyperlipidemic mice. Gastroenterology, 2010. 138(7): p. 2477-86, 2486 e1-3.

58. Houben, T., et al., Blood-derived macrophages prone to accumulate lysosomal lipids trigger oxLDL-dependent murine hepatic inflammation. Sci Rep, 2017. 7(1): p. 12550.

59. Hoppe, G., et al., Products of lipid peroxidation induce missorting of the principal lysosomal protease in retinal pigment epithelium. Biochim Biophys Acta, 2004. 1689(1): p. 33-41.

60. Yuan, X.M., et al., The toxicity to macrophages of oxidized low-density lipoprotein is mediated through lysosomal damage. Atherosclerosis, 1997. 133(2): p. 153-61.

61. Guha, S. and H. Padh, Cathepsins: fundamental effectors of endolysosomal proteolysis. Indian J Biochem Biophys, 2008. 45(2): p. 75-90.

62. Leist, M. and M. Jaattela, Triggering of apoptosis by cathepsins. Cell Death Differ, 2001. 8(4): p. 324-6.

63. Campden, R.I. and Y. Zhang, The role of lysosomal cysteine cathepsins in NLRP3 inflammasome activation. Arch Biochem Biophys, 2019. 670: p. 32-42.

64. Lassen, K.G. and R.J. Xavier, Mechanisms and function of autophagy in intestinal disease. Autophagy, 2018. 14(2): p. 216-220.

65. Vidak, E., et al., Cysteine Cathepsins and their Extracellular Roles: Shaping the Microenvironment. Cells, 2019. 8(3).

66. Walenbergh, S.M., et al., Plasma cathepsin D correlates with histological classifications of fatty liver disease in adults and responds to intervention. Sci Rep, 2016. 6: p. 38278.

67. Benes, P., V. Vetvicka, and M. Fusek, Cathepsin D--many functions of one aspartic protease. Crit Rev Oncol Hematol, 2008. 68(1): p. 12-28.

68. Moallem, S.A., et al., Correlation between cathepsin D serum concentration and carotid intima-media thickness in hemodialysis patients. International Urology and Nephrology, 2011. 43(3): p. 841-848.

69. Liu, L., et al., Increased Cathepsin D Correlates with Clinical Parameters in Newly Diagnosed Type 2 Diabetes. Dis Markers, 2017. 2017: p. 5286408.

70. Dubey, V. and S. Luqman, Cathepsin D as a Promising Target for the Discovery of Novel Anticancer Agents. Curr Cancer Drug Targets, 2017. 17(5): p. 404-422.

71. Hausmann, M., et al., Cathepsin D is up-regulated in inflammatory bowel disease macrophages. Clin Exp Immunol, 2004. 136(1): p. 157-67.

72. Houben, T., et al., Cathepsin D regulates lipid metabolism in murine steatohepatitis. Sci Rep, 2017. 7(1): p. 3494. 


\section{Chapter 1}

73. Seematter, G., et al., Relationship between stress, inflammation and metabolism. Curr Opin Clin Nutr Metab Care, 2004. 7(2): p. 169-73.

74. Schneiderman, N., G. Ironson, and S.D. Siegel, Stress and health: psychological, behavioral, and biological determinants. Annu Rev Clin Psychol, 2005. 1: p. 607-28.

75. Kuo, L.E., et al., Chronic stress, combined with a high-fat/high-sugar diet, shifts sympathetic signaling toward neuropeptide $Y$ and leads to obesity and the metabolic syndrome. Ann N Y Acad Sci, 2008. 1148: p. 232-7.

76. Kiecolt-Glaser, J.K., Stress, food, and inflammation: psychoneuroimmunology and nutrition at the cutting edge. Psychosom Med, 2010. 72(4): p. 365-9.

77. Liu, Y.Z., Y.X. Wang, and C.L. Jiang, Inflammation: The Common Pathway of Stress-Related Diseases. Front Hum Neurosci, 2017. 11: p. 316.

78. Perez-Martinez, P., et al., Lifestyle recommendations for the prevention and management of metabolic syndrome: an international panel recommendation. Nutr Rev, 2017. 75(5): p. 307-326.

79. Beavers, K.M., T.E. Brinkley, and B.J. Nicklas, Effect of exercise training on chronic inflammation. Clin Chim Acta, 2010. 411(11-12): p. 785-93.

80. Salas-Salvado, J., et al., The role of diet in the prevention of type 2 diabetes. Nutr Metab Cardiovasc Dis, 2011. 21 Suppl 2: p. B32-48.

81. Yamamoto, M., et al., Restriction of dietary calories, fat and iron improves non-alcoholic fatty liver disease. J Gastroenterol Hepatol, 2007. 22(4): p. 498-503.

82. Hannah, W.N., Jr. and S.A. Harrison, Lifestyle and Dietary Interventions in the Management of Nonalcoholic Fatty Liver Disease. Dig Dis Sci, 2016. 61(5): p. 1365-74.

83. Ahmed, I.A., et al., Lifestyle interventions for non-alcoholic fatty liver disease. Saudi J Biol Sci, 2019. 26(7): p. 15191524.

84. Lassailly, G., et al., Bariatric Surgery Reduces Features of Nonalcoholic Steatohepatitis in Morbidly Obese Patients. Gastroenterology, 2015. 149(2): p. 379-88; quiz e15-6.

85. Ertek, S. and A. Cicero, Impact of physical activity on inflammation: effects on cardiovascular disease risk and other inflammatory conditions. Arch Med Sci, 2012. 8(5): p. 794-804.

86. Eckard, C., et al., Prospective histopathologic evaluation of lifestyle modification in nonalcoholic fatty liver disease: a randomized trial. Therap Adv Gastroenterol, 2013. 6(4): p. 249-59.

87. Janczura, M., et al., The Relationship of Metabolic Syndrome with Stress, Coronary Heart Disease and Pulmonary Function--An Occupational Cohort-Based Study. PLoS One, 2015. 10(8): p. e0133750.

88. Sun, Y., et al., Stress Triggers Flare of Inflammatory Bowel Disease in Children and Adults. Front Pediatr, 2019. 7: p. 432.

89. Zelber-Sagi, S., J. Godos, and F. Salomone, Lifestyle changes for the treatment of nonalcoholic fatty liver disease: a review of observational studies and intervention trials. Therap Adv Gastroenterol, 2016. 9(3): p. 392-407.

90. Murphy, L.R., Stress management in work settings: a critical review of the health effects. Am J Health Promot, 1996. 11(2): p. 112-35.

91. Sumida, Y., et al., Phase 3 drug pipelines in the treatment of non-alcoholic steatohepatitis. Hepatol Res, 2019. 49(11): p. 1256-1262.

92. Bril, F., et al., Response to Pioglitazone in Patients With Nonalcoholic Steatohepatitis With vs Without Type 2 Diabetes. Clin Gastroenterol Hepatol, 2018. 16(4): p. 558-566 e2.

93. Zezos, P. and E.L. Renner, Liver transplantation and non-alcoholic fatty liver disease. World J Gastroenterol, 2014. 20(42): p. 15532-8.

94. Taylor, S. and A.J. Lobo, Diagnosis and treatment of inflammatory bowel disease. Practitioner, 2016. 260(1795): p. 1923.

95. Nielsen, O.H. and L.K. Munck, Drug insight: aminosalicylates for the treatment of IBD. Nat Clin Pract Gastroenterol Hepatol, 2007. 4(3): p. 160-70.

96. Waljee, A.K., et al., Corticosteroid Use and Complications in a US Inflammatory Bowel Disease Cohort. PLoS One, 2016. 11(6): p. e0158017.

97. Paramsothy, S., et al., The current state of the art for biological therapies and new small molecules in inflammatory bowel disease. Mucosal Immunol, 2018. 11(6): p. 1558-1570.

98. Hwang, J.M. and M.G. Varma, Surgery for inflammatory bowel disease. World J Gastroenterol, 2008. 14(17): p. 267890.

99. Baigent, C., et al., Efficacy and safety of cholesterol-lowering treatment: prospective meta-analysis of data from 90,056 participants in 14 randomised trials of statins. Lancet, 2005. 366(9493): p. 1267-78.

100. Zimmer, S., et al., Cyclodextrin promotes atherosclerosis regression via macrophage reprogramming. Sci Transl Med, 2016. 8(333): p. 333ra50.

101. Kantner, I. and R.G. Erben, Long-term parenteral administration of 2-hydroxypropyl-beta-cyclodextrin causes bone loss. Toxicol Pathol, 2012. 40(5): p. 742-50.

102. Kramer, L., D. Turk, and B. Turk, The Future of Cysteine Cathepsins in Disease Management. Trends Pharmacol Sci, 2017. 38(10): p. 873-898.

103. Lucaciu, L.A., R. Seicean, and A. Seicean, Small molecule drugs in the treatment of inflammatory bowel diseases: which one, when and why? - a systematic review. Eur J Gastroenterol Hepatol, 2020. 32(6): p. 669-677. 
104. Santhekadur, P.K., D.P. Kumar, and A.J. Sanyal, Preclinical models of non-alcoholic fatty liver disease. J Hepatol, 2018. 68(2): p. 230-237.

105. Bieghs, V., et al., LDL receptor knock-out mice are a physiological model particularly vulnerable to study the onset of inflammation in non-alcoholic fatty liver disease. PLoS One, 2012. 7(1): p. e30668.

106. Ishibashi, S., et al., Hypercholesterolemia in low density lipoprotein receptor knockout mice and its reversal by adenovirus-mediated gene delivery. J Clin Invest, 1993. 92(2): p. 883-93.

107. Zhong, F., et al., Rodent Models of Nonalcoholic Fatty Liver Disease. Digestion, 2019: p. 1-14.

108. Lieber, C.S., et al., Model of nonalcoholic steatohepatitis. Am J Clin Nutr, 2004. 79(3): p. 502-9. 



\section{Chapter 2}

The Ins and Outs of Cathepsins: Physiological function and role in disease management 


\title{
Chapter 2
}

\begin{abstract}
Cathepsins are the most abundant lysosomal proteases that are mainly found in acidic endo/lysosomal compartments where they play a vital role in intracellular protein degradation, energy metabolism, and immune responses among a host of other functions. The discovery that cathepsins are secreted and remain functionally active outside of the lysosome has caused a paradigm shift. Contemporary research has unraveled many versatile functions of cathepsins in extra lysosomal locations including cytosol and extracellular space. Nevertheless, extracellular cathepsins are majorly upregulated in pathological states and are implicated in a wide range of diseases including cancer and cardiovascular diseases. Taking advantage of the differential expression of the cathepsins during pathological conditions, much research is focused on using cathepsins as diagnostic markers and therapeutic targets. A tailored therapeutic approach using selective cathepsin inhibitors is constantly emerging to be safe and efficient. Moreover, recent development of proteomic-based approaches for the identification of novel physiological substrates offers a major opportunity to understand the mechanism of cathepsin action. In this review, we summarize the available evidence regarding the role of cathepsins in health and disease, discuss their potential as biomarkers of disease progression, and shed light on the potential of extracellular cathepsin inhibitors as safe therapeutic tools.
\end{abstract}




\section{Introduction}

Lysosomes are intracellular membrane-bound organelles characterized by an acidic interior and harbor a variety of hydrolytic enzymes including lipases, proteases and glycosidases that participate in cellular catabolism [1,2]. The functions of most of these enzymes require an acidic lumen, which is maintained by the vacuolar H+ ATPase (V-ATPase), an ATP-driven proton pump located on the lysosomal transmembrane. Lysosomes can fuse with endosomes, phagosomes, autophagosomes and break down both endogenous and exogenous cargo consisting of various biomolecules such as lipids, proteins, polysaccharides, and certain pathogens. Additionally, lysosomes play critical roles in some of the most vital processes such as metabolic signaling, repair of the plasma membrane and nutrient sensing [3-5]. Lysosomes perform this multitude of coordinated events with the help of their enzymes.

Among the variety of enzymes that lysosomes harbor, cathepsins are a family of lysosomal proteases with an astonishingly broad spectrum of functions. Cathepsins help in intracellular housekeeping where they for example, participate in the antigen processing during immune responses and degrade several proteases and chemokines to maintain cellular homeostasis (reviewed in [6,7]). Mammalian proteases have been classified into 5 different families namely metallo, serine, threonine, aspartic, and cysteine proteases based on the type of amino acid at the active site. All cathepsins fall into three different protease families viz; serine proteases (cathepsins $A$ and $G$ ), aspartic proteases (cathepsin $D$ and $E$ ) and eleven cysteine cathepsins (cathepsins $B, C, F, H, K, L, O, S, V, X$, and $W$ ) $[6,8,9]$. Serine proteases constitute up to $31 \%$ of total proteases expressed in human body, while cysteine and aspartic proteases make up for $25 \%$ and $4 \%$ of the total protease population respectively $[6,10]$. Cathepsins show highest activity in the low pH environment of lysosomes. However, certain cathepsins are also found to be active outside of their optimal pH range of $5[6,11]$. The pH optimum of cathepsin S was found to be $6.5[12,13]$. While cathepsin D shows optimal activity at pH 4, its activity was detected even at $\mathrm{pH}$ of 7.4 (although at reduced kinetic rates) [14]. Moreover, cathepsin $\mathrm{K}$ and $\mathrm{H}$ displayed stable activity at $\mathrm{pH} 7$ [15] indicating their wide range of proteolytic activity. Due to the retained activity far outside the optimal pH range cathepsins have been identified with specific proteolytic functions even outside of the endo/lysosomal system $[16,17]$.

Although cathepsins exhibit some level of similarity in their proteolytic functions in physiological processes, consequences of cathepsin dysfunction are very diverse in terms of clinical symptoms. Deregulated cathepsin synthesis and activity has been associated with several diseases including the metabolic syndrome, cancer and inflammatory neurological diseases (reviewed in [18]). Cathepsins are known to activate and/or degrade several important neuronal proteins, and thus have important roles in neurodegenerative disorders (reviewed in $[19,20]$ ). For example, cathepsin D plays an important role in neuronal cell homeostasis whose dysfunction leads to impaired proteolysis of target proteins such as huntingtin, $\alpha$-synuclein, tau, lipofuscin, apoE resulting in Huntington's and Parkinson's amongst other neurological disorders [20]. In addition to cathepsin D, several cathepsins are associated with inflammatory neurological diseases including Niemann-Pick type $C$ (NPC) disease, neuronal ceroid lipofuscinosis (NCL) and Alzheimer's (reviewed in [21-23]). Cardiovascular disorders such as cardiomyopathy, hypertension, myocardial infarction, atherosclerosis and aortic aneurysms are characterized by extensive extracellular matrix (ECM) degradation and remodeling, one of prime processes mediated by cathepsins (reviewed in [24]). Similarly, in cancer, tumors 
metastasize by ECM degradation and cathepsins are known to breakdown the constituents of ECM, epithelial membrane and cell-cell junctions facilitating cancer cell migration. Further, cathepsins are involved in growth, invasion, angiogenesis, and therapeutic resistance associated with tumors [25]. Obesity and diabetes are most common metabolic disorders and cathepsins $\mathrm{L}, \mathrm{S}$, and $\mathrm{K}$ are found to have potential roles in these pathologies (reviewed in [26]). Thus, the range and complexity of biological activities reliant on cathepsins make them a common point of interest in diverse diseases. Importantly, owing to their differential function during physiological and pathological conditions, cathepsins are considered as highly relevant targets for therapeutic intervention in this range of diseases.

This review is divided into four parts. In the first part, we provide a basic overview of cathepsin expression and function within the endo/lysosomal compartments. In the next two parts, we emphasize on the mechanisms leading to the translocation of cathepsins into the cytosol and extracellular milieu, respectively. Finally, this review further highlights the prospect of using highly specific, targeted cathepsin inhibitors for clinical implementation in various diseases.

\section{Cathepsins in lysosomes}

Cathepsins exhibit similarities in their cellular localization and biosynthesis with some differences in their expression pattern. Of all the lysosomal proteases, cathepsins $L, B$, and $D$ are the most abundant with their lysosomal concentrations equivalent to $1 \mathrm{mM}$ [27]. Cathepsins B, H, L, C, X, V, and $\mathrm{O}$ are ubiquitously expressed while cathepsins $\mathrm{K}, \mathrm{S}, \mathrm{E}$, and $\mathrm{W}$ show cell or tissue-specific expression. Cathepsin $\mathrm{K}$ is expressed in the osteoclasts (multinucleated cells of bone) and in epithelial cells. Cathepsins $\mathrm{S}, \mathrm{E}$, and $\mathrm{W}$ are mainly expressed in immune cells.

\subsection{Regulation}

Cathepsins undergo transcriptional, translational, post-translational and epigenetic regulation. At transcriptional level, except for cathepsin $D$ all other cathepsins show TATA-independent transcription initiation which defines the transcription initiation site. Cathepsin L, K, C, and B are known to require various transcription factors such as nuclear factor (NF-Y), specificity proteins, Sp1, Sp3 and erythroblast transformation-specific (Ets) family factors for transcription initiation and regulation $[28,29]$. Cathepsin $D$ is known to be transcriptionally regulated by Peroxisome ProliferatorActivated Receptor $\gamma$ in dendritic cells [30] and by estrogen in breast cancer cells [31]. Transcript variants for cathepsin $B$ and $L$ have been reported as a result of alternative splicing. These transcripts differ in their mRNA stability and thus are known to be accumulated during tumors [32]. Cathepsin L is found to be translationally regulated by internal ribosomal entry site (IRES) [33]. Cysteine cathepsins are known to also contain CpG islands in their promoter region and thus are epigenetically regulated by methylation [25]. Additionally, cathepsin enzyme activity is regulated by $\mathrm{pH}$ and endogenous protein inhibitors such as stefins, cystatins, and kiniogens [8]. 


\subsection{Synthesis}

The primary structure of all cathepsins consists of a signal peptide, a propeptide, and a catalytically active mature functional enzyme. Cathepsins are synthesized as preprocathepsins in the endoplasmic reticulum (ER). A N-terminal signal peptide of 20-25 amino acids directs preprocathepsins into the ER lumen where they are cleaved co-translationally by a signal peptidase generating procathepsins, the less active zymogen forms. Simultaneously, N-linked glycosylation occurs within the ER, producing high levels of mannose in the procathepsins [34]. Procathepsins later travel through the Golgi stocks where the mannose residues are modified to mannose-6-phosphate moieties (M6P). The M6P-tagged procathepsins are recognized and bound by M6P receptors in the trans-Golgi network (TGN) and are either directly sorted to the endo/lysosomes or first pass the plasma membrane from where they enter the endo/lysosomes in an indirect manner. Both direct and indirect routes rely on clathrin-coated vesicles to carry the cathepsins from the TGN or plasma membrane to the endo/lysosomes. Once the cathepsins are trafficked to endo/lysosomes, free M6P receptor is transported back to the TGN [35]. Inside lysosomes cleavage of propeptide converts procathepsins to mature active cathepsins. This activation occurs via different modes such as auto-activation or trans-activation or both. During the auto activation the $\mathrm{pH}$ inside the lysosomes enables the cleavage of propeptide by the catalytic site of the same enzyme, while trans-activation requires help of other proteases. For instance, cathepsin $\mathrm{B}, \mathrm{H}, \mathrm{L}, \mathrm{S}$, and $\mathrm{K}$ are activated by auto-activation, while cathepsins $C$ and $X$ need cathepsins $L$ and $S$ for their activation $[36,37]$. Cathepsin $D$ is found to be processed by partial auto-activation and requires cathepsin $B$ and $L$ for further maturation [38]. Further, procathepsin $D$ is known to undergo a two-step maturation process in the endo/lysosomal compartments: in the first step, propeptide is partially cleaved to generate an active single-chain intermediate which upon reaching lysosome undergoes second processing in the region of T155K173 to yield a double-chain mature cathepsin D [39].

\subsubsection{M6P-independent sorting}

While most of the lysosomal enzymes are dependent on M6P for their transport, several studies have reported the existence of $\mathrm{M} 6 \mathrm{P}$-independent transport routes of cathepsins to reach endo/lysosomes with the aid of alternative receptors. One such alternative receptor protein is sortilin, a transmembrane Golgi protein, which was shown to be involved in the sorting of cathepsin $\mathrm{H}$ and cathepsin $D$ in COS-7 cells [40]. In mouse embryonic fibroblasts cathepsin B and D are known to be captured by the membrane proteins LRP1 (low-density lipoprotein receptor-related protein 1) and LDLR (lowdensity lipoprotein receptor) $[41,42]$. While procathepsin $D$ is known to complex with sphingolipid activator precursor protein prosaposin, procathepsin $B$ is known to rely on its membrane association to enter lysosomes independent of M6P route [43-45]. Recently, type 1 transmembrane protein SEZ6L2 was linked with sorting of cathepsin D in neurons [46]. Thus, cell-type, post-translational processing, and modifications play an important role in the sorting of cathepsins towards lysosomes.

\subsection{Physiological functions of cathepsins in the endo/lysosomes}

Cathepsins carry out many proteolytic events in the compartments of the endocytic pathway thus contributing to the protein turn-over and normal metabolism of the cell. The $\mathrm{pH}$ inside the 
endo/lysosomal compartment favors cathepsin activity while it induces conformational changes in the substrates leading to their cleavage by cathepsins, and thereby helping cathepsins to successfully degrade the cargo transported to the endo/lysosomes $[9,47]$.

\subsubsection{Immune responses}

Cathepsins display significant roles predominantly in the endosomes of immune cells. They are known to participate both in the innate and adaptive immune responses. During the innate immune responses, lysosomal cathepsins have been shown to cleave the ectodomains of Toll-like receptors (TLRs) 7 and 9 that are expressed on endo/lysosomal membranes, where TLRs recognize nucleic acids of phagocytosed microbes. The processed forms of TLR 7 and 9 then recruit the adaptor protein MyD88 leading to activation of TLR signaling pathways [48]. During adaptive immune responses, $T$ lymphocytes recognize processed antigens on the surface of antigen-presenting cells (APCS) that are bound to major histocompatibility complex (MHC) molecules. While MHC class I molecules present processed antigenic peptides that are derived from the cytosol, MHC class II molecules present peptides derived from the endo/lysosomal compartment. Further, $\mathrm{MHC}$ class II molecules require an invariant chain (li), a glycoprotein necessary for their folding and assembly. Various cathepsins are found to be involved in the (I) proteolytic processing of antigens into short peptides and (II) degradation of the invariant chain thus facilitating adaptive immune responses $[49,50]$. However, Deussing et al. [51] demonstrated that cathepsins B and D are non-essential in MHC II mediated antigen presentation.

\subsubsection{Autophagy}

Cathepsins participate in autophagy, an essential catabolic process which delivers cytosolic constituents to lysosomes for their subsequent degradation in order to maintain cell homeostasis [52]. As demonstrated by Dennemarker et al. [53], cathepsin L deficient primary mouse embryonic fibroblasts showed normal initiation of the autophagy process, autophagosome formation as well as autophagosome-lysosome fusion but impaired degradation of autolysosomal content. Despite this impairment of autolysosomal turnover due to the lack of cathepsin L, the viability of the cells was not affected. This implies that autolysosomal degradation is not solely dependent on cathepsin $L$ and is likely compensated by cathepsin $D$ as evidenced by the increased levels of cathepsin $D$ in cathepsin $L$ knock out cells. In addition, cathepsins also regulate lysosome and autophagosome populations. Cathepsin B is known to degrade the calcium channel MCOLN1/TRPML1 in the lysosomes, leading to the suppression of transcription factor TFEB and thus inhibiting the expression of autophagy-related proteins. This response is known to keep in check the lysosomal biogenesis and population of cellular autophagosomes [54]. Cathepsin $\mathrm{S}$ is required for the fusion processes of autophagosomes and lysosomes and its deficiency results in increased number of autophagosomes. Moreover, cathepsin S-mediated autophagic flux is known to induce M2-type polarization of tumor associated macrophages, which would then contribute to tumor [55]. 


\subsubsection{Growth and development related functions}

Cathepsins regulate growth and development related processes by processing various hormones and growth factors. For instance, cathepsin B degrades a variety of substrates including insulin-like growth factor-1 (IGF-I), glucagon, pituitary hormone, thyroglobulin [56,57]. Additionally, cathepsin B in the adipocytes is known to cleave perilipin 1 (PLIN1), a lipid droplet-associated protein leading to increased lipolysis in obese adipose tissue [58]. Further, the proteolytic events regulated by cathepsins are critical in the control of biological processes including ovulation, neuronal development and fertilization. For instance, cathepsin $L$ is one of the key proteases upregulated in granulosa cells of ovulatory follicles mediating follicular rupture during ovulation [59]. Cathepsin D is known to process vitellogenin and together with $B$ and $L$, is involved in oogenesis in lower vertebrates. Cathepsins are extensively involved in embryo development. Relevantly, knock down of cathepsin D led to tissue defects including eye, skin and swim bladder in zebra fish [60] and caused intestinal mucosa damage, atrophy of myelin sheath and early death in mice [61]. Similarly, mice deficient in cathepsins $B$ and $L$ developed atrophy in the cerebral and cerebellar regions of the brain, suggesting their necessity for neuronal development [62].

\subsection{Pathological role of cathepsins in the endo/lysosomes}

Inactivation or loss-of-function of cathepsins results in inappropriate degradation and abnormal accumulation of substrates in lysosomes leading to various diseases including lysosomal storage disorders (LSD). Further, defective proteolytic events by cathepsins can lead to several LSDs, namely, galactosialidosis [63] neuronal ceroid lipofuscinosis (CLN) type 10 and 13 [63,64], Papillon-Lefèvre syndrome [65], pycnodysostosis [66], and Alzheimer's disease [21]. Further, given the important role of cathepsins in autophagy, impaired proteolysis by cathepsins can lead to massive accumulation of autophagosomes which eventually can cause pathologies including cellular senescence, inflammasome activation [67] and Saposin (Sap) C deficiency, a rare variant form of Gaucher disease [68]. Impaired activity of Cathepsin D and L would result in accumulation of $\alpha$-synuclein amyloid fibrils in the brain tissue leading to synucleinopathies [69]. Alterations or loss of functions in the cathepsin activity are known to have adverse effects on reproduction and fertility [70]. Thus, consequences of cathepsin inactivation translate to a wide variety of clinical complications. Detailed functions of individual cathepsins and the associated pathologies are mentioned in Table 1.

Table 1: Cathepsins in the endo/lysosomal compartment

\begin{tabular}{|c|c|c|c|c|c|c|}
\hline Cathepsin & $\begin{array}{c}\text { Enzyme } \\
\text { Commission } \\
\text { Number }\end{array}$ & $\begin{array}{l}\text { Catalytic } \\
\text { type }\end{array}$ & Function & Pathology & OMIM ID & Reference \\
\hline Cathepsin A & 3.4.16.5 & serine & $\begin{array}{c}\text { dual function: } \\
\text { a. protective: } \beta \text {-galactosidase and } \\
\text { neuraminidase } \\
\text { b. degradative: bioactive peptides } \\
\text { like bradykinin, angiotensin, } \\
\text { oxytocin, endothelin } 1\end{array}$ & $\begin{array}{l}\text { hypertension } \\
\text { Galactosialidosis }\end{array}$ & 256540 & [63] \\
\hline Cathepsin B & 3.4 .22 .1 & cysteine & $\begin{array}{l}\text { degrades amyloid- } \beta \text {; activation of } \\
\text { pro-hormones and pro-enzymes; } \\
\text { trypsin activation; } \\
\text { promotes viral entry into cells }\end{array}$ & $\begin{array}{c}\text { Alzheimer's; } \\
\text { gaucher disease } \\
\text { acute pancreatitis }\end{array}$ & & {$[21,68]$} \\
\hline Cathepsin C & 3.4.14.1 & cysteine & $\begin{array}{l}\text { inflammatory responses and } \\
\text { activation of serine proteases }\end{array}$ & $\begin{array}{c}\text { Papillon-Lefèvre } \\
\text { syndrome } \\
\text { Periodontitis }\end{array}$ & 245000 & [65] \\
\hline
\end{tabular}




\begin{tabular}{|c|c|c|c|c|c|c|}
\hline & & & $\begin{array}{l}\text { including neutrophil elastase and } \\
\text { cathepsin G }\end{array}$ & & & \\
\hline $\begin{array}{l}\text { Cathepsin } \\
\text { D }\end{array}$ & 3.4 .23 .5 & aspartic & $\begin{array}{l}\text { embryo and neuronal development } \\
\text { brain antigen processing of } \alpha- \\
\text { Synuclein; tau, amyloid } \beta \text {, apoE; } \\
\text { degradation of hormones, } \\
\text { proenzymes and growth factors }\end{array}$ & $\begin{array}{l}\text { Alzheimer's } \\
\text { disease; } \\
\text { CLN } 10 \\
\text { Parkinson's; } \\
\text { Huntington's }\end{array}$ & 610127 & {$[20,61,64]$} \\
\hline Cathepsin E & 3.4.23.34 & aspartic & $\begin{array}{c}\text { carboxypeptidase } \mathrm{A} \text { and IgE } \\
\text { processing }\end{array}$ & atopic dermatitis & & [71] \\
\hline Cathepsin F & 3.4.22.41 & cysteine & $\begin{array}{l}\text { li chain processing and MHC-II class } \\
\text { responses }\end{array}$ & CLN 13 & 615362 & [63] \\
\hline $\begin{array}{l}\text { Cathepsin } \\
\text { G }\end{array}$ & 3.4.21.20 & serine & auto antigen processing & $\begin{array}{c}\text { auto-immune } \\
\text { diseases }\end{array}$ & & [72] \\
\hline $\begin{array}{c}\text { Cathepsin } \\
\mathrm{H}\end{array}$ & 3.4 .22 .16 & cysteine & prohormone processing & type 1 diabetes & & [9] \\
\hline Cathepsin K & 3.4 .22 .38 & cysteine & $\begin{array}{l}\text { TLR signaling; processing of } \beta \text { - } \\
\text { endorphin in brain }\end{array}$ & $\begin{array}{l}\text { periodontitis; } \\
\text { pycnodysostosis }\end{array}$ & 265800 & {$[73,74]$} \\
\hline Cathepsin L & 3.4.22.15 & cysteine & $\begin{array}{l}\text { antigen and li chain processing; } \\
\text { prohormone processing; } \\
\text { degradation of } \alpha \text {-Synuclein, tau; } \\
\text { promotes viral entry into cells }\end{array}$ & $\begin{array}{l}\text { Parkinson's } \\
\text { disease; } \\
\text { Frontotemporal } \\
\text { dementia }\end{array}$ & & {$[19,49]$} \\
\hline Cathepsin S & 3.4 .22 .27 & cysteine & $\begin{array}{c}\text { antigen processing and } \\
\text { presentation; } \\
\text { li chain processing }\end{array}$ & $\begin{array}{l}\text { auto-immune } \\
\text { diseases }\end{array}$ & & {$[50]$} \\
\hline Cathepsin X & 3.4 .18 .1 & cysteine & T-cell migration and invasion & - & & [75] \\
\hline $\begin{array}{c}\text { Cathepsin } \\
\text { O }\end{array}$ & 3.4 .22 .42 & cysteine & - & - & & \\
\hline Cathepsin V & 3.4 .22 .43 & cysteine & $\begin{array}{l}\text { natural killer cell and CD8+ } \\
\text { cytotoxic cell production }\end{array}$ & thymic pathology & & {$[76]$} \\
\hline $\begin{array}{c}\text { Cathepsin } \\
\mathrm{W}\end{array}$ & 3.4.22.- & cysteine & $\begin{array}{l}\text { component of endoplasmic } \\
\text { reticulum proteolytic machinery }\end{array}$ & - & & {$[77]$} \\
\hline Cathepsin Z & 3.4 .18 .1 & cysteine & intracellular protein turnover & - & & [78] \\
\hline
\end{tabular}

- implies that no function or pathology is yet discovered for the respective cathepsins. CLN stands for ceroid lipofuscinosis, neuronal; OMIM stands for online mendelian inheritance in man.

\section{Cathepsins in cytosol}

As described in the previous section, proteolytic functions of cathepsins mainly occur in the endo/lysosomal compartments. However, most of the cathepsins are released into the extralysosomal locations such as cytosol, nucleus, and mitochondria where they perform crucial tasks. In the current section, we describe the mechanism of cathepsin translocation into different regions of the cell and their respective functions.

\subsection{Mechanism of translocation}

Perhaps one of the most simplistic mechanisms though which cathepsins are translocated to cellular compartments other than lysosomes is by leaking outside of lysosomes. Lysosomes are surrounded by a limiting membrane, which is a phospholipid bilayer that is characterized by variety of integral membrane proteins, which together protect the organelle from the degradative enzymes [79]. Damage to the lysosomal membrane components or disturbances in membrane fluidity and structure can influence lysosomal stability and subsequently lead to rupture of lysosomes, a process called lysosomal membrane permeabilization (LMP). LMP is induced by a plethora of stimuli including oxidative stress, lysosomotropic agents, as well as some endogenous cell death effectors. While partial permeabilization of the lysosomal membrane induces apoptosis (programmed cell death), complete lysosomal rupture leads to necrosis (reviewed in [80]). 


\subsubsection{Oxidative stress induced LMP}

Lysosomal destabilization is recognized feature during oxidative stress-mediated cell damage. The initial burst of reactive oxygen species (ROS) may be generated by different triggers inside or outside of the lysosomes. For example, ceramide accumulation or ingestion of heavy metals such as silica or asbestos is known to increase NAPDH oxidase in the lysosomes to generate ROS. The generated free radicals interact with free intralysosomal iron forming highly reactive hydroxyl radicals in a Fentontype reaction [80]. Such hydroxyl radicals induce LMP by causing lipid peroxidation of lysosomal membranes thereby forming lipofuscins and further damaging lysosomal membrane proteins (reviewed in [81]). An oxidative burst elicited by interferon- $\gamma$ was reported to induce LMP and cathepsin release [82]. Further, ROS is known to modify lipids and lysosomal trapping of oxLDL has the potential to damage and disrupt the lysosomal membrane leading to the secretion of cathepsins $[83,84]$. Additionally, ROS is found to be generated outside of the lysosomes by destabilized mitochondria, inhalation of exogenous pollutants and ionizing radiation leading to LMP (reviewed in $[85,86])$.

\subsubsection{Lysosomotropic agents}

Lysosome-targeting agents, referred to as lysosomotropic agents and lipid detergents cause LMP either by direct membrane lysis or by osmotic lysis of lysosomal membrane. Examples of agents that cause direct membrane lysis include O-methyl-serine dodecylamide hydrochloride (MSDH), $\mathrm{N}$ dodecylimidazole, $n-\beta$-naphthylamide, and endogenous compounds such as lipofuscin. Due to their amphiphilic nature, these agents partition between the water phase and the phospholipid bilayer of lysosomal membrane. Further, accumulation of the lysosomotropic agents and molecules leads to thinning of the lysosomal bilayer and subsequent solubilization of lysosomal membrane (LM) (reviewed in [87]). Lysosomotropic amines, such as chloroquine or ammonium chloride and peptides such as (LeuLeu)nOMe are known to change the permeability of LMs and thus leading to increased influx of solutes into lysosomes and eventually leading to osmotic lysis [88]. Loss of membrane cholesterol or modification of membrane lipids by lipases such as sphingomyelinase and ceramidase are known to induce LMP by increasing the lysosomal permeability for potassium ions and protons and subsequent osmotic lysis $[89,90]$. Thus, various molecules can cause LMP, leading to leakage of cathepsins into the cytosol.

\subsection{Transport of cathepsins to different regions of cytosol}

Besides LMP, other mechanisms such as alternative translation or exon skipping can lead to extra lysosomal translocation of cathepsins. Translation initiation at a different start site produces cathepsins which are devoid of signal peptide, while exon skipping generates truncated cathepsins with modified signal sequences thus misrouting cathepsins from their biosynthetic pathway to locations such as cytosol, nucleus, and mitochondria. For instance, translational initiation at downstream AUG site is known to localize cathepsin $L$ in nucleus [91] and alternative splicing in cathepsin B mRNA with missing exons at 2 and 3 is known to direct cathepsin B to mitochondria [92], Another report by Bestvater et al. [93] proposed an alternative targeting signal besides the usual $\mathrm{N}$ - 
terminal signal peptide for cathepsin B namely, a signal patch within its heavy chain domain that facilitates its nuclear import.

\subsection{Physiological functions of cathepsins in the cytosol}

Although cathepsins do not retain optimal activity at the neutral $\mathrm{pH}$ of the cytosol, their proteolytic activity is known to be preserved by substrate binding and acidification of the cytosol observed under certain conditions like apoptosis and cathepsins in the cytosol are known to mediate key physiological processes as described below.

\subsubsection{Apoptosis and necroptosis}

Apoptosis is a highly regulated, fundamental physiological process of cell death responsible for removal of damaged/aging cells by activation of caspase family of proteins. Apoptosis involves two different pathways; intrinsic pathway, also known as the mitochondria pathway, or the extrinsic pathway involving death ligands (reviewed in [94]). The mitochondria pathway is regulated by B-cell lymphoma-2 (Bcl-2) family proteins. Bcl-2 family consists of both anti-apoptotic members such as Bcl2 and $\mathrm{Bcl}-\mathrm{xL}$ and pro-apoptotic proteins including Bax, Bak, and Bid [95]. By genetic modification or use of pharmacological inhibitors, several studies have established that cathepsins in the cytosol play an important role in apoptosis, specifically by activating other apoptotic proteases [96]. Cytosolic cathepsins $B, D$, and $L$ are implicated in the degradation of Bid, resulting in its activation and translocation to mitochondria. This translocation leads to cytochrome $C$ release from mitochondria followed by caspase activation, and thus initiating apoptotic cell death. Simultaneously, cathepsins are involved in the degradation of anti-apoptotic proteins $\mathrm{Bcl}-2, \mathrm{Bcl}-\mathrm{xL}, \mathrm{Mcl}-1$, and XIAP (X-linked inhibitor of apoptosis), promoting apoptosis [97]. Additionally, in T cells, cathepsin-D-mediated apoptosis involves the activation of Bax, and the release of apoptosis-inducing factor (AIF) and cystatin c. This process has been shown to be independent of Bid cleavage and initiates apoptosis by directly activating the initiator caspase-8 [98-100].

Recent studies have shown that cathepsins regulate programmed necrosis termed necroptosis. Necroptosis is initiated by various stimuli and requires the kinase activity of receptor-interacting serine/ threonine kinase1 (Rip1). In macrophages, it has been reported that cathepsins B and S are known to cleave receptor-interacting protein 1 (Rip1) kinase and thus limit macrophage necroptosis [101].

\subsubsection{Inflammation}

Cytosolic cathepsins are shown to be involved in mediating inflammatory responses via activation of inflammasomes. The NLRP3 inflammasome is a multi-protein complex which is activated upon bacterial infections, LMP or cellular damage. Upon activation, pro-caspase1 is converted to active caspase 1 by autocatalysis. Active caspase 1 then proceeds to cleave the cytokine precursors pro IL$1 \beta$ and IL-18 into their mature secreted forms (reviewed in [102]). Gene knockout and siRNA knockdown of cathepsins $B, C, S, L$, and $Z$ resulted in a suppression of IL-1 $\beta$ secretion [103-105]. However, cathepsins are not shown to directly cleave either caspase-1 or IL-1 $\beta$ suggesting that they 
operate upstream of inflammasome activation [103]. Additionally, Cathepsins $Z$ and $S$ are known to compensate for the activity of cathepsins B, C, and L in LMP-mediated inflammasome activation by unknown mechanisms [106].

\subsubsection{Functions of nuclear cathepsins}

Cathepsins in the nucleus are known to process transcription factors that control cell cycle progression thus facilitating cell proliferation and differentiation (reviewed in [107]. The $\mathrm{CDP} / \mathrm{Cux} /$ Cut transcription factors are a group of highly conserved proteins in higher eukaryotes that are involved in cell cycle proliferation, particularly in the transition from $\mathrm{G} 1$ to $\mathrm{S}$ phase. Cathepsin $\mathrm{L}$ in the nucleus is known to cleave the CDP/Cux, which accelerates progression into $S$ phase of the cell cycle [108]. Nuclear cathepsin D promotes cell proliferation by acting as co-factor for Tricho-rhinophalangeal syndrome Type 1 (TRPS1) transcription factor and is known to enhance mammary gland differentiation by cleaving histone H3 [109]. Nuclear cathepsins are known to regulate transforming growth factor- $\beta$ (TGF- $\beta$ ) signaling, an important pathway normal growth and tissue development whose mis regulation can lead to carcinogenesis. The downstream effectors of TGF- $\beta$ signaling, the Smad proteins, are phosphorylated and activated by receptors such as importin $\beta$ which mediate pSmad nuclear translocation, where they regulate transcription. Cathepsins $B, K, L$, and $S$ are known to localize to nuclear membrane where they exert differential effects in the translocation of pSMAD2 and pSMAD3 proteins by modulating importin $\beta$ expression and thus regulate TGF- $\beta$ signaling [110]. Physiological functions of cytosolic cathepsins are listed in Table $\mathbf{2}$.

Table 2: Function of cathepsins in the cytosol

\begin{tabular}{cccc}
\hline Cathepsin & $\begin{array}{c}\text { Extra lysosomal } \\
\text { location }\end{array}$ & Function & Reference \\
\hline Cathepsin B, D and L & cytosol & proteolytic processing of Bid during \\
apoptosis & [97,111] & {$[103-106]$} \\
\hline Cathepsin B, C, L, S and Z & cytosol & NLRP3 inflammasome activation & [112] \\
\hline Cathepsin B & cytosol & $\begin{array}{c}\text { regulation of hepatic lipid metabolism by } \\
\text { degrading liver fatty acid binding protein }\end{array}$ & [91] \\
\hline Cathepsin L and H & nucleus & cell cycle regulation & [110] \\
\hline Cathepsin B, K, L and S & nucleus & TGF- $\beta$ signaling & [113] \\
\hline Cathepsin B & nucleus & bile-salt induced apoptosis & - \\
\hline W, Z * & - & & \\
\hline
\end{tabular}

* Roles for these remaining cathepsins outside of the lysosome have not yet been reported.

\subsection{Pathological functions of cathepsins in the cytosol}

Lysosomal disruption and the subsequent release of cathepsins in the cytosol can have detrimental effects. For instance, though apoptosis is a highly regulated fundamental physiological process, there are many pathological conditions including neurodegeneration and ischemia that involve excessive apoptosis in which cytosolic cathepsins are known to play an active role $[96,114]$. Accordingly, substantial evidence supports the contribution of cathepsins from ruptured lysosomes in the pathology of many neurodegenerative diseases [115]. In contrast to healthy brain, in Alzheimer's brain, cathepsin $D$ is found in the cytosol and such cytosolic cathepsin $D$ is known to cleave tau protein generating truncated form of tau which in turn forms paired helical filaments leading to 
neurofibrillary degradation [115]. Cytosolic cathepsin $D$ is also implicated in glaucoma by promoting apoptosis in trabecular mesh work cells, which maintain intraocular pressure of the eye [116]. Furthermore, cytosolic cathepsin D has been proposed as a biomarker of age-related neurodegenerative disorders [117]. In line, it has been found that cathepsin D translocation into the cytosol led to pronounced age-related changes in rats, by increasing the degeneration of neurons $[117,118]$. NLRP3 inflammasome is essential for defending against bacterial infections and mis regulated NLRP3 inflammasome has been implicated in metabolic inflammatory disorders including type 2 diabetes, atherosclerosis, heart reperfusion injuries, and chronic kidney diseases (reviewed in [119]). Given the known role of cathepsins in modulating inflammasome activation it then also indirectly suggests a pathological relevance of cathepsins in these disorders.

Another cytosolic cathepsin that is associated with disease conditions is cathepsin L. In pathological conditions such as proteinuria and glomerular kidney disease, translocation of cathepsin L to cytosol has been documented [120]. Here, in contrast to its lysosomal counterpart, cytosolic cathepsin L in podocytes (cells in the Bowman's capsule of the kidneys), is known to degrade cytoskeleton proteins, namely, CD2-associated protein, synaptopodin and dynamin, thus leading to the reorganization of the actin cytoskeleton, proteinuria and subsequent renal failure [121]. Nuclear cathepsin L activity is associated with polycystic kidney disease [121] and alterations in its activity significantly influenced colorectal cancer disease progression [108]. Nuclear cathepsin F activity is found to be correlated with markers of transcriptional regulation in hepatic stellate cells [122]. Mitochondrial procathepsin $B$ is known to induce morphological changes in mitochondrial integrity and thus can lead to cell death [123]. Taken together, cathepsins perform dynamic functions outside of the lysosomes based on their cytosolic location.

\section{Cathepsins in the extracellular space}

Numerous lines of evidence demonstrated the presence of cathepsins in the extracellular space. Although the extracellular localization of cathepsins is more commonly observed during pathological conditions, cathepsins are mostly involved in the bone remodeling and plasma membrane repair during physiological conditions.

\subsection{Mechanism of translocation}

Cathepsins are normally secreted via lysosomal exocytosis or by alternative sorting from Golgi. Usually, secretion of cathepsins is often accompanied by their over expression which is commonly observed in cancer and inflammatory conditions [16]. Immune cells are known to secrete high levels of cathepsins. Additionally, osteoclasts, keratinocytes, thyroid cells, and smooth muscle cells also release cathepsins into the extracellular space [124].

\subsubsection{Lysosomal exocytosis of cathepsins}

The secretory pathway of lysosomes, known as lysosomal exocytosis, has been reported in many types of cells and is induced by various stimuli such as wound, cellular stress, cancer, or by signals from cytokines [125]. The induction of exocytosis occurs by the recruitment of lysosomes to the 
periphery of the cells. This movement of lysosomes is mediated along microtubule track with the help of various kinesins and a multi subunit complex named BLOC-one-related complex (BORC) that promotes kinesin-mediated lysosome movement toward the cell periphery [126]. The movement of lysosomes to the periphery is followed by docking, where the lysosome and plasma membrane are brought into closer contact with the help of soluble $\mathrm{N}$-ethylmaleimide-sensitive factor attachment protein receptor (SNARE) complexes, namely the interaction of v-SNARE (vamp7) on lysosomal membrane with t-SNARE (synaxin 4 and SNAP-23) on the cytoplasmic side of the plasma membrane (PM) [127]. Both lysosomes and PM contain negatively charged lipids in their outer and inner layers, respectively. Hence, cells rely on calcium ions $\left(\mathrm{Ca}^{2+}\right)$ to bridge the opposing charges on the membranes. Further, lysosomal docking and fusion are regulated by transcription factor EB (TFEB). EB modulates lysosomal exocytosis by triggering intracellular $\mathrm{Ca}^{2+}$ elevation through the endo/lysosomal cation-channel mucolipin 1 (MCOLN1) [128]. Finally, the fusion of lysosomes with the PM results in the secretion of lysosomal components into the extracellular space. Castro-Gomes et al. [129] elegantly demonstrated that cathepsin B and $L$ are released extracellularly by lysosomal exocytosis and participate in PM repair using in vitro systems.

\subsubsection{Alternative sorting of cathepsins into extracellular space}

As described in the section 2.1 of this review, cathepsins are trafficked to endo/lysosomal compartments of the cell with the help of M6P receptors. Changes in the $\mathrm{pH}$ are known to disrupt the recycling of $\mathrm{M} 6 \mathrm{P}$ to the Golgi, where its absence leads to the potential re-routing of procathepsins into the extracellular space directly or packaged into the secretory vesicles $[37,130]$. In contrast, during conditions such as bone resorption, secretion of active form of cathepsins is observed suggesting that the secretion is context dependent. Once secreted, cathepsins can be found attached to the caveolae on the PM, as seen in case of cathepsin B [131] or released in the extracellular space directly or packed inside the secretory vesicles.

\subsection{Physiological function of extracellular cathepsins}

Cathepsins require acidic $\mathrm{pH}$ for their optimum activity in contrast to the neutral $\mathrm{pH}$ found in extracellular space. However, cathepsins are often secreted as less active procathepsins which are normally stable at neutral $\mathrm{pH}$ [132]. For cathepsins secreted in the mature active form, vacuolar $\mathrm{H+-}$ ATPases (V-ATPase) are known to provide a local acidic hub in the pericellular to facilitate their prolonged activity in the extracellular space [133]. For instance, in bone lacunae, V-ATPase and the chloride channel create a low $\mathrm{pH}$ extracellular environment with the help of $\mathrm{H}+$ and $\mathrm{Cl}$ - ion flow [134]. Cathepsins in the extracellular matrix are known to degrade many components of the ECM and thus participate in physiological processes including wound healing [135], bone remodeling $[136,137]$ and processing of prohormones [8].

\subsubsection{ECM degradation}

ECM is composed of fibrous proteins such as elastin, collagen, proteoglycans, and fibronectins that form the intricate meshwork for holding the cells embedded within the tissues Further, ECM undergoes constant remodeling and ECM components have several binding sites for growth factors 
that ultimately control cell adhesion, proliferation, migration, and polarity [138]. ECM conducts mechanical signals that further activate cytoskeletal and intracellular signaling pathways. In healthy tissue, ECM homeostasis is mainly regulated by cathepsins and matrix metalloproteases (reviewed in [139]).

Bone remodeling is a continuous process that involves bone resorption and remodeling performed by specialized cells called osteoclasts. Further, bone and cartilage predominantly contain type 1 collagen. During bone resorption, osteoclasts attach to the surface of bone leading to the creation of an extracellular compartment which is isolated from general extracellular fluid (reviewed in [140]). Numerous studies showed that the active cathepsin $\mathrm{K}$ released from osteoclasts into the resorption lacunae is known to degrade type 1 collagen and elastin and thus plays a pivotal role in bone resorption $[17,137,139,141]$. Moreover, cathepsins are known to degrade two bone ECM proteins, osteocalcin, and osteonectin. While osteocalcin is involved in bone formation and insulin metabolism, osteonectin helps in cell matrix interactions [139,142]. Further, proteoglycans are major constituents of ECM are composed of core protein with covalently attached glycosaminoglycan molecules (GAGs). Various cathepsins are known to cleave the protein core of proteoglycan. For example, cathepsins B and L cleave perlecan, a heparan sulfate proteoglycan, generating LG3 peptide, which is known to have neuroprotective role in brain ischemia $[143,144]$.

\subsubsection{Functions of cathepsins on the plasma membrane}

Cathepsins on the cell surface are known to be involved in plasma membrane repair. Cathepsin B released from keratinocytes attaches to cells surface where it is known to be involved in keratinocyte migration by degrading components of ECM during wound healing [145]. Another cathepsin that translocate to the plasma membrane is cathepsin $X$ which helps with processes of cell adhesion and signaling [75]. $\beta$ integrins are cell surface proteins that help in cell adhesion and invasion. Cathepsin $X$ on the plasma membrane is known to cleave regulatory motifs in two different types of $\beta 2$ integrin receptors. By activating $\beta 2$ integrin receptor Mac-1, cathepsin $X$ enhances the adhesion of immature dendritic cells to ECM, leading to their activation. Additionally, cathepsin $X$ activates another $\beta 2$ integrin receptor, LFA-1 enhancing the proliferation of $T$ lymphocytes thus accelerating immune responses $[75,146]$.

\subsubsection{Functions of cathepsins in the secretory vesicles}

Neurons and endocrine cells carry out cell-cell communication with the help of peptide neurotransmitters. Secretory vesicles in these cells provide regulated secretion of neurotransmitters, which are first synthesized as inactive prohormones. Proteolytic processing of the proproteins or prohormones occurs in the secretory vesicles by cathepsins [147]. Numerous studies have identified cathepsin $L$ and $V$ as key processing enzymes for production of numerous peptide neurotransmitters including neuropeptide $Y$, enkephalin, cholecystokinin, and dynorphins $[25,148]$. The environment inside the vesicles is known to have acidic $\mathrm{pH}$ which promotes the function of cathepsins to generate active peptides $[149,150]$. 


\subsection{Pathological functions of extracellular cathepsins}

While ECM remodeling is an important physiological process, aberrant ECM dynamics can lead to uncontrolled cell proliferation, invasion, and differentiation leading to fatal pathologies including atherosclerosis, cancer, and tissue fibrosis [138]. Extracellular cathepsin activity have been implicated in many of these diseases as elaborated below.

\subsubsection{Cancer}

Cancer results from abnormal cell proliferation in the body. Increased or abnormal proteolytic activity of cathepsins is known to degrade ECM components facilitating migration and invasion of tumors leading to malignancy (reviewed in [124]). Cathepsins can be secreted from cancer cells and infiltrating immune cells called tumor-associated macrophages (TAMs). Over secretion of cathepsins is associated with their abnormal expression. Several molecular factors that regulate expression of cathepsins in tumor microenvironment are defined. Signal transducer and activator of transcription 3 and 6 (STAT3, STAT6) are known to promote the secretion of procathepsins B, C, S, and Z mainly from macrophages [151]. Collagen 1 is known to induce secretion of procathepsin $B$ by regulating Ets1 transcription factor [152]. Additionally, it has been postulated that cancer microenvironment downregulates M6P receptor mRNA due to overexpression of cathepsins. Due to its weak affinity towards M6P receptor, cathepsin L is known to be directly secreted from TGN before binding to M6P receptor in fibrosarcoma cells [153]. Metastatic tumor cells are known to have defective lysosomal sorting of procathpesin $D$ resulting in its secretion. Further, the acidic tumor microenvironment not only favors the maturation of procathepsins but also promotes their activity [154].

Elevated levels of extracellular cathepsins have been identified in various cancers such as breast, lung, colon, pancreas, skin, prostate, bladder, ovary, and head and neck [25]. In addition to increased levels, increased activity of cathepsins is often associated with activation of tumor-associated cytokines, shedding and cleaving cell-cell adhesion molecules, thereby destroying cell contact and contributing to metastasis. For example, E-cadherin is an important cell adhesion molecule and epithelial tumor suppressor. Extracellular cathepsins $B, L$ and $S$ are known to cleave $E$-cadherin promoting tumor invasion into surrounding tissues [155]. In contrast, secreted cathepsin $D$ is known to cleave $23 \mathrm{kDa}$ prolactin, a lactogenic hormone produced by pituitary gland to a $16 \mathrm{kDa}$ fragment which has antiangiogenic properties in rat mammary epithelial cells [156] and in bovine corpus luteum but not in humans [157]. Another mechanism through which extracellular cathepsins contribute to cancer progression is by shedding, a process by which proteases release the extracellular domains of cell surface proteins from the cells [158]. Shedding converts membraneassociated proteins into soluble ones and thus reduces their cell surface expression that in turn is a means of regulation for subsequent physiological processes. Apart from several cell adhesion molecules (CAMs) identified as substrates for shedding, cathepsins also target the Ras signaling pathway, a major intracellular signaling pathway in cancer progression. Extracellular cathepsins $\mathrm{S}$ and $L$ are known to shed plexins and epidermal growth factor receptor (EGFR), both of which are substrates for Ras pathway activation [16,158]. Similarly, extracellular cathepsin D is known to degrade the ECM proteins thus freeing the embedded growth factors such as fibroblast growth factor. Growth factors are complex polypeptides that have critical roles in basement membrane disruption, cell migration, and tumor metastasis in auto and paracrine manner [154]. 


\subsubsection{Metabolic disorders}

Lipoprotein accumulation and metabolism are important contributors to various diseases including cardiovascular diseases and obesity -associated disorders such as non-alcoholic steatohepatitis (NASH). Extracellular cathepsins are found to be involved in transport, efflux, and processing of lipoprotein molecules or their receptors (reviewed in [159]). For instance, extracellular cathepsins $F$, $\mathrm{K}$, and $\mathrm{S}$ are known to degrade cholesterol acceptors on the cell surface, thereby reducing cholesterol efflux and initiating foam cell formation, a key feature of atherosclerosis [24]. Increased presence of low-density lipoprotein (LDL) is a characteristic of both atherosclerosis and NASH. Extracellular cathepsin D is known to proteolytically modify apolipoprotein B-100 (apoB), component of LDL and subsequently leading to LDL accumulation in arterial intima $[160,161]$. In agreement, extracellular cathepsin D inhibition is known to reduce hepatic steatosis [162]. Further, LDL accumulation in the arterial intima can lead to its oxidation which enhances inflammatory responses, that in a positive feedback loop promotes further secretion of cathepsins leading to exacerbation of lipid accumulation and inflammation (reviewed in [163]). Similar to extracellular cathepsin D, extracellular cathepsin S is known to promote inflammation by cleaving chemokines such as fractalkine (CX3CL1) that help in leukocyte migration and neuropathic pain [164]. Finally, extracellular cathepsin activity is involved in lung fibrosis, osteoarthritis, osteoporosis and rheumatoid arthritis which are summarized in Table 3.

Table 3: Cathepsins in the extracellular space

\begin{tabular}{|c|c|c|c|}
\hline Cathepsin & Substrate & Pathological State & Reference \\
\hline Cathepsin B, K, and L & proteoglycan & osteoarthritis & {$[137,165]$} \\
\hline Cathepsin B, L, G, and S & fibronectin & cancer and adipogenesis & [166-169] \\
\hline Cathepsin B, L, and S & laminin & cancer neovascularization, intestinal trauma & [166-168] \\
\hline Cathepsin K & collagen type I & $\begin{array}{l}\text { osteoporosis, rheumatized arthritis, } \\
\text { osteoarthritis }\end{array}$ & [137] \\
\hline Cathepsin B, K, L, and S & collagen type 2 & lung fibrosis, cardiovascular diseases and cancer & {$[138,139]$} \\
\hline Cathepsin B & tenascin & cancer & [170] \\
\hline Cathepsin B, K, L, and S & aggrecan & osteoarthritis & {$[136,171]$} \\
\hline Cathepsin L, S, and B & plexin & tumorigenesis & [158] \\
\hline Cathepsin S & fractalkine & neuropathic pain & [164] \\
\hline Cathepsin D & $\begin{array}{l}\text { fibroblast growth } \\
\text { factor }\end{array}$ & breast cancer & {$[172]$} \\
\hline Cathepsin V & elastin & cancer & [173] \\
\hline Cathepsin X & CXCL-12 & - & {$[174]$} \\
\hline Cathepsin W & - & cell-mediated cytotoxicity & [175] \\
\hline $\begin{array}{c}\text { Cathepsin } A, C, E, F, O \text {, and } \\
Z^{*}\end{array}$ & - & - & \\
\hline
\end{tabular}

* Role of these extracellular cathepsins in pathologies in not known.

The mechanisms involved in the translocation of cathepsins and their respective site-specific functions are illustrated in Figure 1. 


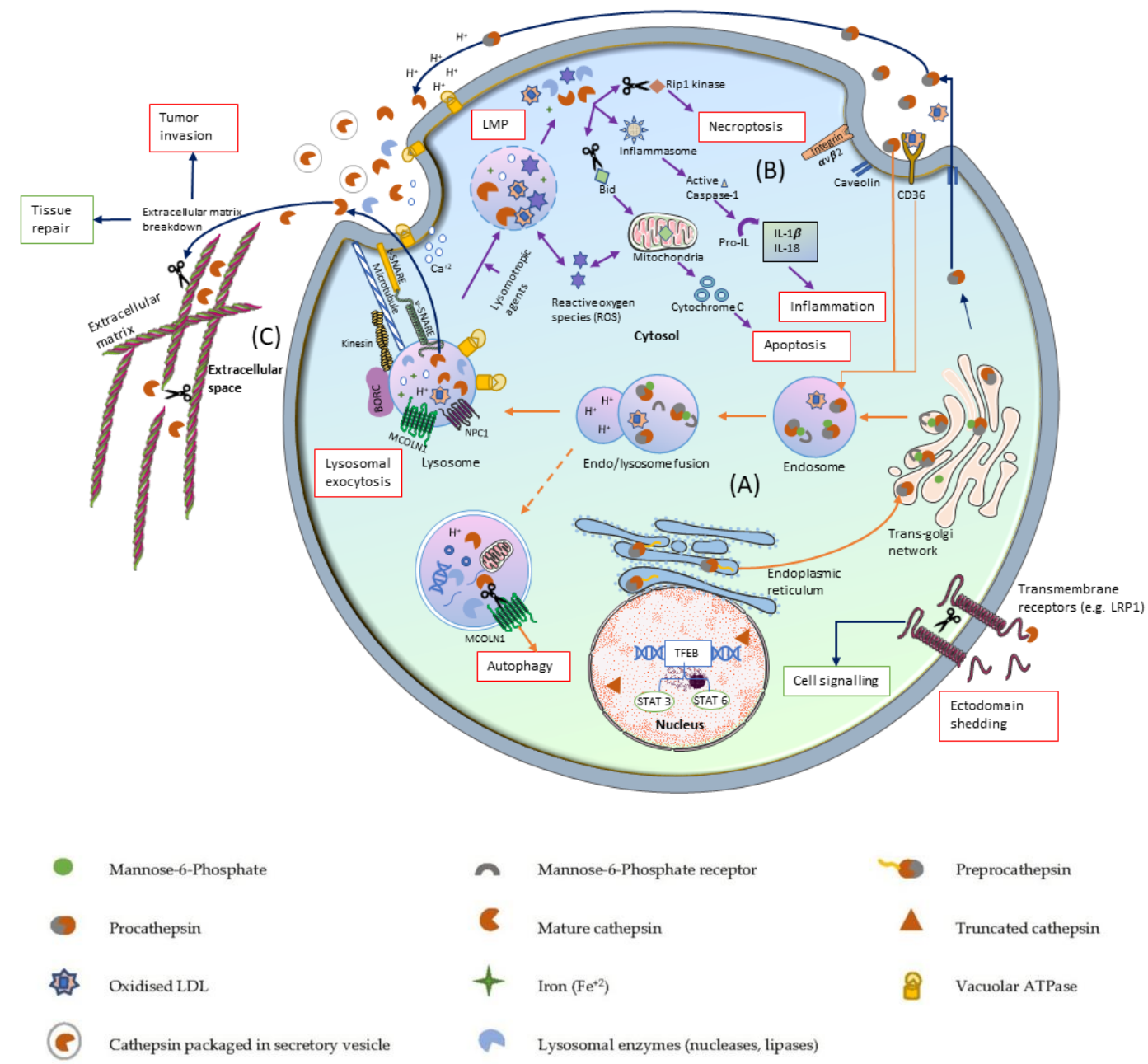

Figure 1 Site-specific functions of cathepsins. (A) cathepsins in the lysosomes (represented by orange arrows): Cathepsins are synthesized as preprocathepsins in the endoplasmic reticulum and transported to endo/lysosomes via Trans-Golgi network where the acidic $\mathrm{pH}$ enables their maturation. Cathepsins in the lysosomes are mostly involved in protein degradation besides participating in autophagy. (B) cathepsins in the cytosol (represented by purple arrows): Lysosomotropic agents, ROS or accumulation of modified lipids (oxLDL) leads to lysosomal membrane permeabilization (LMP), releasing cathepsins into the cytosol. Cytosolic cathepsins participate in various activities. For example, cathepsins trigger the inflammasome and promote apoptosis and necroptosis by cleaving various proteins. (C) Cathepsins in the extracellular space (represented by blue arrows): Lysosomal exocytosis involves the secretion of lysosomal contents into the extracellular space with the help of several protein-receptor interactions and $\mathrm{Ca}^{+2}$ ion gradient. Cathepsins are released in the form of procathepsins or enclosed in the secretory vesicles or as active cathepsins. Secreted cathepsins remain attached to the plasma membrane or are released into the extracellular space. Cathepsins on the plasma membrane cleave proteins like integrins. Secreted cathepsins mainly participate in extracellular matrix degradation and thus help in wound healing. However, excessive ECM cleavage facilitates tumor invasion and promotes cancer. While in the extracellular space cathepsins also shed the ectodomains of transmembrane receptors, leading to either activation or inhibition of cell signaling. ROS: reactive oxygen species; LMP: Iysosomal membrane permeabilization; NPC1: Niemann-Pick disease type C1; CD36: cluster of differentiation 36; Figure is created with permission from Servier Medical Art image bank. 


\section{Targeting cathepsins in disease management}

Localization of cathepsins (endo/lysosomal/cytosolic/extracellular space) governs several aspects of cathepsin function. A wealth of knowledge has been published on the differential expression and functional profiles of cathepsins in various pathologies making them potential diagnostic biomarkers and most desirable therapeutic targets.

Cathepsin levels and activity have been found to be upregulated in sera and tumors of many cancer patients $[176,177]$. For instance, expression and activity levels of cathepsins $B$ and $L$ corelated with breast cancer progression [178]. Furthermore, B and L cathepsins also correlated with relapse rate after treatment in primary breast cancers [178]. In cancers concerning colon, lung, brain and head and neck, concentration of cathepsins $B$ and $L$ within tumors correlated with survival probability [179]. Moreover, cathepsin L levels were increased in sera of patients with pancreatic [180] and liver cancers [181]. Serum cathepsin H levels were found to be increased in patients with lung [182], melanoma [183], and colorectal cancers [184]. The findings of these recent studies have alluded to a potential of utilizing cathepsins as biomarkers of cancer though few studies show some discrepancies in the outcomes of cathepsin expression and activity during cancer (reviewed in [185]). In addition to cancer, plasma levels of cathepsin $\mathrm{S}, \mathrm{K}$, and $\mathrm{L}$ have been proposed as biomarkers in coronary artery disease, aneurysm, adiposity, and peripheral arterial disease [186]. Similarly, plasma cathepsin D levels associated with metabolic alterations in liver during NAFLD [187]. Recent studies suggested that plasma cathepsin D levels correlated with type 2 diabetic patients [188] and moreover plasma cathepsin D activity is suggested as biomarker for hepatic insulin sensitivity [189]. Cathepsins $Z$ and $K$ are proposed diagnostic markers for osteoporosis [190,191]. Another promising development is the use of cathepsins as fluorescent probes in diagnostic non-invasive imaging which has had success in preclinical mouse models [192].

In diseases caused by inactivation or loss-of-function of cathepsins, supplying functional cathepsins could be a valuable means to restore cellular function and ameliorate the disease. For instance, enzyme replacement therapy (ERT) replacing defective lysosomal cathepsin D by recombinant procathepsin D has been proven beneficial for CLN10 [193]. However, due to known associations of excessive extracellular cathepsin $D$ with other pathological conditions highlighted previously in this review caution must be exercised when utilizing this therapeutic approach to avoid overaccumulation of cathepsin $D$ in extra lysosomal locations which could possibly lead to activation of extracellular cathepsin D mediated pathological processes. When implementing the ERT approach for cathepsins, the recombinant cathepsins' mannose-6-phosphate content must be maximized to ensure their optimal uptake by tissues and to avoid their extra lysosomal accumulation. In addition, conjugating recombinant cathepsins with chaperones might help to diffuse them across cell membranes and reach target tissues including central nervous system (CNS) [194]. Additionally, close monitoring of injected levels of recombinant enzyme in patients might help in preventing any negative effects.

Increased understanding of the structure, differential expression and localization of cathepsins in various pathologies has opened a new avenue for the design of small molecule inhibitors of cathepsins with the hope of producing highly specific, targeted drugs for many diseases. Several small molecule inhibitors of cathepsin $\mathrm{S}$ and $\mathrm{K}$ are being tested in clinical trials [192]. Additionally, combinatorial therapies involving cathepsin inhibition are gaining more attention. For example, 
cathepsin inhibitors conjugated with radio/chemotherapy would be a potential anti-cancer treatment [195]. Further, cathepsin K clinical inhibitors for treatment of osteoporosis might have potential to attenuate cancer [196]. Unfortunately, one of the bone-specific cathepsin K inhibitors to complete phase III clinical trials, odanacatib (Merck) had to be discontinued due to risk of stroke [197]. The failure was likely due to the fact that the active-site inhibitor of $K$ also blocked the other essential protease functions of cathepsin K [17]. One of the strategies to alleviate this problem was to identify inhibitors specific to the exosites or allosteric sites, that essentially inhibit the pathological collagenolytic activity only and thus limiting the cytotoxicity [198]. One of such exosite inhibitors of cathepsin K was successfully demonstrated in a mouse model of osteoporosis [199]. However, since not all cathepsin activities are modulated by exosite interactions it presents a limitation for the use of exosite inhibitors in certain cathepsin related pathologies. Other novel ways such as site-specific inhibition of cathepsins would also reduce their off-target limits. For instance, small-molecule inhibitors targeting only the secreted extracellular fraction of cathepsin $D$ and not the lysosomal fraction had beneficial results in a rodent model of NAFLD [162]. Moreover, antibodies against extracellular fraction of cathepsin S efficiently inhibited tumor growth and neovascularization in xenograft tumors [200] and improved chemotherapy efficacy in colorectal carcinomas [201]. There are still a few inhibitors targeting either extracellular cathepsins or their substrates that require further validation in clinical setting [192] as described in Table 4. Taken together, while evidence that shows a direct link between extracellular cathepsins and various pathologies are few, current studies to date show promising therapeutic result of targeting specifically the extracellular fraction of cathepsins in their respective pathologies. Therefore, further research looking into the specific role of extracellular cathepsins and the therapeutic value of targeting them is recommended.

Table 4: List of available extracellular cathepsin inhibitors

\begin{tabular}{ccc}
\hline Inhibitor & Target & Reference \\
\hline Fsn0503 & antibody against extracellular cathepsin S & {$[200]$} \\
\hline Nitroxoline & extracellular cathepsin B & {$[202]$} \\
\hline LNC-NS-629 & extracellular cathepsin B & {$[203]$} \\
\hline CTD-002 & extracellular cathepsin D & {$[162]$} \\
\hline
\end{tabular}

\section{Conclusion}

In conclusion, cathepsins are a group of enzymes with distinct functions at different locations inside and outside of the cells. While many recent findings helped us to further understand these roles and establish the potential for targeting, many mechanistic aspects of cathepsin action are still to be explored. Currently, the extracellular role of cathepsins have gained enormous attention in the biomedical field, establishing them as non-invasive diagnostic markers and pharmacological targets in immune disorders, cancer, osteoarthritis, and metabolic diseases. Many selective cathepsin inhibitors with limited side effects are being developed and have shown success in the preclinical animal models and are awaiting validation in the clinical setting. With the advent of new mass spectrometry technologies and systems biology approaches, the future research should focus to better understand the specific substrates of cathepsins in the physiological and pathological environment. Finally, the future of cathepsins in targeted drug delivery looks more promising than ever. 


\section{References}

1. Perera, R.M.; Zoncu, R. The Lysosome as a Regulatory Hub. Annu. Rev. Cell Dev. Biol. 2016, 32, 223-253, doi:10.1146/annurev-cellbio-111315-125125.

2. de Duve, C. The lysosome turns fifty. Nat. Cell Biol. 2005, 7, 847-849, doi:10.1038/ncb0905-847.

3. Xu, H.; Ren, D. Lysosomal physiology. Annu. Rev. Physiol. 2015, 77, 57-80, doi:10.1146/annurev-physiol-021014071649.

4. Lawrence, R.E.; Zoncu, R. The lysosome as a cellular centre for signalling, metabolism and quality control. Nat. Cell Biol. 2019, 21, 133-142, doi:10.1038/s41556-018-0244-7.

5. Lim, C.Y.; Zoncu, R. The lysosome as a command-and-control center for cellular metabolism. J. Cell Biol. 2016, 214, 653-664, doi:10.1083/jcb.201607005.

6. Turk, V.; Stoka, V.; Vasiljeva, O.; Renko, M.; Sun, T.; Turk, B.; Turk, D. Cysteine cathepsins: From structure, function and regulation to new frontiers. Biochim. Biophys. Acta 2012, 1824, 68-88, doi:10.1016/j.bbapap.2011.10.002.

7. Vasiljeva, O.; Reinheckel, T.; Peters, C.; Turk, D.; Turk, V.; Turk, B. Emerging roles of cysteine cathepsins in disease and their potential as drug targets. Curr. Pharm. Des. 2007, 13, 387-403, doi:10.2174/138161207780162962.

8. Brix, K.; Dunkhorst, A.; Mayer, K.; Jordans, S. Cysteine cathepsins: Cellular roadmap to different functions. Biochimie 2008, 90, 194-207, doi:10.1016/j.biochi.2007.07.024.

9. Patel, S.; Homaei, A.; El-Seedi, H.R.; Akhtar, N. Cathepsins: Proteases that are vital for survival but can also be fatal. Biomed. Pharm. 2018, 105, 526-532, doi:10.1016/j.biopha.2018.05.148.

10. Rossi, A.; Deveraux, Q.; Turk, B.; Sali, A. Comprehensive search for cysteine cathepsins in the human genome. Biol. Chem. 2004, 385, 363-372, doi:10.1515/BC.2004.040.

11. Sanman, L.E.; van der Linden, W.A.; Verdoes, M.; Bogyo, M. Bifunctional Probes of Cathepsin Protease Activity and $\mathrm{pH}$ Reveal Alterations in Endolysosomal pH during Bacterial Infection. Cell Chem. Biol. 2016, 23, 793-804, doi:10.1016/j.chembiol.2016.05.019.

12. Bromme, D.; Bonneau, P.R.; Lachance, P.; Wiederanders, B.; Kirschke, H.; Peters, C.; Thomas, D.Y.; Storer, A.C.; Vernet, T. Functional expression of human cathepsin $S$ in Saccharomyces cerevisiae. Purification and characterization of the recombinant enzyme. J. Biol. Chem. 1993, 268, 4832-4838.

13. Kirschke, H.; Schmidt, I.; Wiederanders, B.; Cathepsin, S. The cysteine proteinase from bovine lymphoid tissue is distinct from cathepsin L (EC 3.4.22.15). Biochem. J. 1986, 240, 455-459, doi:10.1042/bj2400455.

14. Sapolsky, A.I.; Howell, D.S.; Woessner, J.F., Jr. Neutral proteases and cathepsin D in human articular cartilage. J. Clin. Invest. 1974, 53, 1044-1053, doi:10.1172/JCl107641.

15. Naseem, R.H.; Hedegard, W.; Henry, T.D.; Lessard, J.; Sutter, K.; Katz, S.A. Plasma cathepsin D isoforms and their active metabolites increase after myocardial infarction and contribute to plasma renin activity. Basic Res. Cardiol. 2005, 100, 139-146, doi:10.1007/s00395-004-0499-3.

16. Vidak, E.; Javorsek, U.; Vizovisek, M.; Turk, B. Cysteine Cathepsins and their Extracellular Roles: Shaping the Microenvironment. Cells 2019, 8, doi:10.3390/cells8030264.

17. Vizovisek, M.; Fonovic, M.; Turk, B. Cysteine cathepsins in extracellular matrix remodeling: Extracellular matrix degradation and beyond. Matrix Biol 2019, 75-76, 141-159, doi:10.1016/j.matbio.2018.01.024.

18. Reiser, J.; Adair, B.; Reinheckel, T. Specialized roles for cysteine cathepsins in health and disease. J. Clin. Invest. 2010, 120, 3421-3431, doi:10.1172/JCl42918.

19. Stoka, V.; Turk, V.; Turk, B. Lysosomal cathepsins and their regulation in aging and neurodegeneration. Ageing Res. Rev. 2016, 32, 22-37, doi:10.1016/j.arr.2016.04.010.

20. Vidoni, C.; Follo, C.; Savino, M.; Melone, M.A.; Isidoro, C. The Role of Cathepsin D in the Pathogenesis of Human Neurodegenerative Disorders. Med. Res. Rev. 2016, 36, 845-870, doi:10.1002/med.21394.

21. Cermak, S.; Kosicek, M.; Mladenovic-Djordjevic, A.; Smiljanic, K.; Kanazir, S.; Hecimovic, S. Loss of Cathepsin B and L Leads to Lysosomal Dysfunction, NPC-Like Cholesterol Sequestration and Accumulation of the Key Alzheimer's Proteins. Plos ONE 2016, 11, e0167428, doi:10.1371/journal.pone.0167428.

22. Pislar, A.; Kos, J. Cysteine Cathepsins in Neurological Disorders. Mol. Neurobiol. 2014, 49, 1017-1030, doi:10.1007/s12035-013-8576-6.

23. Nagai, A.; Murakawa, Y.; Terashima, M.; Shimode, K.; Umegae, N.; Takeuchi, H.; Kobayashi, S. Cystatin C and cathepsin B in CSF from patients with inflammatory neurologic diseases. Neurology 2000, 55, 1828-1832, doi:Doi 10.1212/Wnl.55.12.1828.

24. Lutgens, S.P.M.; Cleutjens, K.B.J.M.; Daemen, M.J.A.P.; Heeneman, S. Cathepsin cysteine proteases in cardiovascular disease. FASEB J. 2007, 21, 3029-3041, doi:10.1096/fj.06-7924com.

25. Mohamed, M.M.; Sloane, B.F. Cysteine cathepsins: Multifunctional enzymes in cancer. Nat. Rev. Cancer 2006, 6, 764775, doi:10.1038/nrc1949.

26. Naour, N.; Rouault, C.; Fellahi, S.; Lavoie, M.E.; Poitou, C.; Keophiphath, M.; Eberle, D.; Shoelson, S.; Rizkalla, S.; Bastard, J.P.; et al. Cathepsins in Human Obesity: Changes in Energy Balance Predominantly Affect Cathepsin S in Adipose Tissue and in Circulation. J. Clin. Endocrinol. Metab. 2010, 95, 1861-1868, doi:10.1210/jc.2009-1894.

27. Turk, B.; Turk, D.; Turk, V. Lysosomal cysteine proteases: More than scavengers. Biochim. Biophys. Acta 2000, 1477, 98-111, doi:10.1016/s0167-4838(99)00263-0. 
28. Jean, D.; Guillaume, N.; Frade, R. Characterization of human cathepsin L promoter and identification of binding sites for NF-Y, Sp1 and Sp3 that are essential for its activity. Biochem. J. 2002, 361, 173-184, doi:10.1042/02646021:3610173.

29. Yan, S.; Berquin, I.M.; Troen, B.R.; Sloane, B.F. Transcription of human cathepsin B is mediated by Sp1 and Ets family factors in glioma. DNA Cell Biol. 2000, 19, 79-91, doi:10.1089/104454900314591.

30. Nakken, B.; Varga, T.; Szatmari, I.; Szeles, L.; Gyongyosi, A.; Illarionov, P.A.; Dezso, B.; Gogolak, P.; Rajnavolgyi, E.; Nagy, L. Peroxisome Proliferator-Activated Receptor gamma-Regulated Cathepsin D Is Required for Lipid Antigen Presentation by Dendritic Cells. J. Immunol. 2011, 187, 240-247, doi:10.4049/jimmunol.1002421.

31. Cavailles, V.; Augereau, P.; Rochefort, H. Cathepsin D gene is controlled by a mixed promoter, and estrogens stimulate only TATA-dependent transcription in breast cancer cells. Proc. Natl. Acad. Sci. USA 1993, 90, 203-207, doi:10.1073/pnas.90.1.203.

32. Berquin, I.M.; Sloane, B.F. Cathepsin B expression in human tumors. Adv. Exp. Med. Biol. 1996, 389, 281-294, doi:10.1007/978-1-4613-0335-0_35.

33. Mittal, S.; Mir, R.A.; Chauhan, S.S. Post-transcriptional regulation of human cathepsin L expression. Biol. Chem. 2011, 392, 405-413, doi:10.1515/BC.2011.039.

34. Braulke, T.; Bonifacino, J.S. Sorting of lysosomal proteins. Bba-Mol. Cell Res. 2009, 1793, 605-614, doi:10.1016/j.bbamcr.2008.10.016.

35. Coutinho, M.F.; Prata, M.J.; Alves, S. Mannose-6-phosphate pathway: A review on its role in lysosomal function and dysfunction. Mol. Genet. Metab 2012, 105, 542-550, doi:10.1016/j.ymgme.2011.12.012.

36. Verma, S.; Dixit, R.; Pandey, K.C. Cysteine Proteases: Modes of Activation and Future Prospects as Pharmacological Targets. Front. Pharm. 2016, 7, 107, doi:10.3389/fphar.2016.00107.

37. Roberts R. Lysosomal cysteine proteases: structure, function and inhibition of cathepsins. Drug News Perspect. 2005, 10, 605-614, doi:10.1358/dnp.2005.18.10.949485.

38. Laurent-Matha, V.; Derocq, D.; Prebois, C.; Katunuma, N.; Liaudet-Coopman, E. Processing of human cathepsin D is independent of its catalytic function and auto-activation: Involvement of cathepsins L and B. J. Biochem. 2006, 139, 363-371, doi:10.1093/jb/mvj037.

39. Follo, C.; Castino, R.; Nicotra, G.; Trincheri, N.F.; Isidoro, C. Folding, activity and targeting of mutated human cathepsin D that cannot be processed into the double-chain form. Int J. Biochem. Cell Biol. 2007, 39, 638-649, doi:10.1016/j.biocel.2006.11.010.

40. Canuel, M.; Korkidakis, A.; Konnyu, K.; Morales, C.R. Sortilin mediates the lysosomal targeting of cathepsins D and H. Biochem. Biophys. Res. Commun. 2008, 373, 292-297, doi:10.1016/j.bbrc.2008.06.021.

41. Derocq, D.; Prebois, C.; Beaujouin, M.; Laurent-Matha, V.; Pattingre, S.; Smith, G.K.; Liaudet-Coopman, E. Cathepsin $\mathrm{D}$ is partly endocytosed by the LRP1 receptor and inhibits LRP1-regulated intramembrane proteolysis. Oncogene 2012, 31, 3202-3212, doi:10.1038/onc.2011.501.

42. Markmann, S.; Thelen, M.; Cornils, K.; Schweizer, M.; Brocke-Ahmadinejad, N.; Willnow, T.; Heeren, J.; Gieselmann, V.; Braulke, T.; Kollmann, K. Lrp1/LDL Receptor Play Critical Roles in Mannose 6-Phosphate-Independent Lysosomal Enzyme Targeting. Traffic 2015, 16, 743-759, doi:10.1111/tra.12284.

43. C., C.R.I. The transport of soluble lysosomal hydrolases from the Golgi complex to lysosomes. In The Golgi Apparatus Springer: Vienna, Austria, 2008; doi.org/10.1007/978-3-211-76310-0_25pp. 402-413.

44. Gopalakrishnan, M.M.; Grosch, H.W.; Locatelli-Hoops, S.; Werth, N.; Smolenova, E.; Nettersheim, M.; Sandhoff, K.; Hasilik, A. Purified recombinant human prosaposin forms oligomers that bind procathepsin $D$ and affect its autoactivation. Biochem. J. 2004, 383, 507-515, doi:10.1042/BJ20040175.

45. Tanaka, Y.; Tanaka, R.; Kawabata, T.; Noguchi, Y.; Himeno, M. Lysosomal cysteine protease, cathepsin B, is targeted to lysosomes by the mannose 6-phosphate-independent pathway in rat hepatocytes: Site-specific phosphorylation in oligosaccharides of the proregion. J. Biochem. 2000, 128, 39-48, doi:10.1093/oxfordjournals.jbchem.a022728.

46. Boonen, M.; Staudt, C.; Gilis, F.; Oorschot, V.; Klumperman, J.; Jadot, M. Cathepsin D and its newly identified transport receptor SEZ6L2 can modulate neurite outgrowth. J. Cell Sci. 2016, 129, 557-568, doi:10.1242/jcs.179374.

47. Bright, N.A.; Davis, L.J.; Luzio, J.P. Endolysosomes Are the Principal Intracellular Sites of Acid Hydrolase Activity. Curr Biol 2016, 26, 2233-2245, doi:10.1016/j.cub.2016.06.046.

48. Creasy, B.M.; McCoy, K.L. Cytokines regulate cysteine cathepsins during TLR responses. Cell Immunol 2011, 267, 5666, doi:10.1016/j.cellimm.2010.11.004.

49. Bird, P.I.; Trapani, J.A.; Villadangos, J.A. Endolysosomal proteases and their inhibitors in immunity. Nat. Rev. Immunol. 2009, 9, 871-882, doi:10.1038/nri2671.

50. Guncar, G.; Pungercic, G.; Klemencic, I.; Turk, V.; Turk, D. Crystal structure of MHC class II-associated p41 li fragment bound to cathepsin $L$ reveals the structural basis for differentiation between cathepsins $L$ and $S$. EMBO J. 1999, 18, 793-803, doi:10.1093/emboj/18.4.793.

51. Deussing, J.; Roth, W.; Saftig, P.; Peters, C.; Ploegh, H.L.; Villadangos, J.A. Cathepsins B and D are dispensable for major histocompatibility complex class II-mediated antigen presentation. Proc. Natl. Acad. Sci. USA 1998, 95, 4516-4521, doi:10.1073/pnas.95.8.4516.

52. Ward, C.; Martinez-Lopez, N.; Otten, E.G.; Carroll, B.; Maetzel, D.; Singh, R.; Sarkar, S.; Korolchuk, V.I. Autophagy, lipophagy and lysosomal lipid storage disorders. Biochim. Biophys. Acta 2016, 1861, 269-284, doi:10.1016/j.bbalip.2016.01.006. 


\section{Chapter 2}

53. Dennemarker, J.; Lohmuller, T.; Muller, S.; Aguilar, S.V.; Tobin, D.J.; Peters, C.; Reinheckel, T. Impaired turnover of autophagolysosomes in cathepsin L deficiency. Biol. Chem. 2010, 391, 913-922, doi:10.1515/BC.2010.097.

54. Man, S.M.; Kanneganti, T.D. Regulation of lysosomal dynamics and autophagy by CTSB/cathepsin B. Autophagy 2016, 12, 2504-2505, doi:10.1080/15548627.2016.1239679.

55. Yang, M.; Liu, J.; Shao, J.; Qin, Y.; Ji, Q.; Zhang, X.; Du, J. Cathepsin S-mediated autophagic flux in tumor-associated macrophages accelerate tumor development by promoting M2 polarization. Mol. Cancer 2014, 13, 43, doi:10.1186/1476-4598-13-43.

56. Authier, F.; Kouach, M.; Briand, G. Endosomal proteolysis of insulin-like growth factor-I at its C-terminal D-domain by cathepsin B. FEBS Lett.. 2005, 579, 4309-4316, doi:10.1016/j.febslet.2005.06.066.

57. Guha, S.; Padh, H. Cathepsins: Fundamental effectors of endolysosomal proteolysis. Indian J. Biochem. Biophys 2008, 45, 75-90.

58. Kovsan, J.; Ben-Romano, R.; Souza, S.C.; Greenberg, A.S.; Rudich, A. Regulation of adipocyte lipolysis by degradation of the perilipin protein: Nelfinavir enhances lysosome-mediated perilipin proteolysis. J. Biol. Chem. 2007, 282, 2170421711, doi:10.1074/jbc.M702223200.

59. Robker, R.L.; Russell, D.L.; Espey, L.L.; Lydon, J.P.; O'Malley, B.W.; Richards, J.S. Progesterone-regulated genes in the ovulation process: ADAMTS-1 and cathepsin L proteases. Proc. Natl. Acad. Sci. USA 2000, 97, 4689-4694, doi:10.1073/pnas.080073497.

60. Follo, C.; Ozzano, M.; Mugoni, V.; Castino, R.; Santoro, M.; Isidoro, C. Knock-down of cathepsin D affects the retinal pigment epithelium, impairs swim-bladder ontogenesis and causes premature death in zebrafish. Plos ONE 2011, 6, e21908, doi:10.1371/journal.pone.0021908.

61. Saftig, P.; Hetman, M.; Schmahl, W.; Weber, K.; Heine, L.; Mossmann, H.; Koster, A.; Hess, B.; Evers, M.; von Figura, K.; et al. Mice deficient for the lysosomal proteinase cathepsin $D$ exhibit progressive atrophy of the intestinal mucosa and profound destruction of lymphoid cells. EMBO J. 1995, 14, 3599-3608.

62. Felbor, U.; Kessler, B.; Mothes, W.; Goebel, H.H.; Ploegh, H.L.; Bronson, R.T.; Olsen, B.R. Neuronal loss and brain atrophy in mice lacking cathepsins B and L. Proc. Natl. Acad. Sci. USA 2002, 99, 7883-7888, doi:10.1073/pnas.112632299.

63. Ketterer, S.; Gomez-Auli, A.; Hillebrand, L.E.; Petrera, A.; Ketscher, A.; Reinheckel, T. Inherited diseases caused by mutations in cathepsin protease genes. FEBS J. 2017, 284, 1437-1454, doi:10.1111/febs.13980.

64. Siintola, E.; Partanen, S.; Stromme, P.; Haapanen, A.; Haltia, M.; Maehlen, J.; Lehesjoki, A.E.; Tyynela, J. Cathepsin D deficiency underlies congenital human neuronal ceroid-lipofuscinosis. Brain 2006, 129, 1438-1445, doi:10.1093/brain/awl107.

65. Hart, T.C.; Hart, P.S.; Bowden, D.W.; Michalec, M.D.; Callison, S.A.; Walker, S.J.; Zhang, Y.Z.; Firatli, E. Mutations of the cathepsin C gene are responsible for Papillon-Lefevre syndrome. J. Med. Genet. 1999, 36, 881-887.

66. Ho, N.; Punturieri, A.; Wilkin, D.; Szabo, J.; Johnson, M.; Whaley, J.; Davis, J.; Clark, A.; Weiss, S.; Francomano, C. Mutations of CTSK result in pycnodysostosis via a reduction in cathepsin K protein. J. Bone Min. Res. 1999, 14, 16491653, doi:10.1359/jbmr.1999.14.10.1649.

67. Mizunoe, Y.; Sudo, Y.; Okita, N.; Hiraoka, H.; Mikami, K.; Narahara, T.; Negishi, A.; Yoshida, M.; Higashibata, R.; Watanabe, S.; et al. Involvement of lysosomal dysfunction in autophagosome accumulation and early pathologies in adipose tissue of obese mice. Autophagy 2017, 13, 642-653, doi:10.1080/15548627.2016.1274850.

68. Tatti, M.; Motta, M.; Di Bartolomeo, S.; Scarpa, S.; Cianfanelli, V.; Cecconi, F.; Salvioli, R. Reduced cathepsins B and D cause impaired autophagic degradation that can be almost completely restored by overexpression of these two proteases in Sap C-deficient fibroblasts. Hum. Mol. Genet. 2012, 21, 5159-5173, doi:10.1093/hmg/dds367.

69. McGlinchey, R.P.; Lee, J.C. Cysteine cathepsins are essential in lysosomal degradation of alpha-synuclein. Proc. Natl. Acad. Sci. USA 2015, 112, 9322-9327, doi:10.1073/pnas.1500937112.

70. Carnevali, O.; Cionna, C.; Tosti, L.; Lubzens, E.; Maradonna, F. Role of cathepsins in ovarian follicle growth and maturation. Gen. Comp. Endocrinol. 2006, 146, 195-203, doi:10.1016/j.ygcen.2005.12.007.

71. Tsukuba, T.; Yamamoto, K. [Atopic dermatitis and cathepsin E]. Nihon Yakurigaku Zasshi 2003, 122, 15-20, doi:10.1254/fpj.122.15.

72. Adkison, A.M.; Raptis, S.Z.; Kelley, D.G.; Pham, C.T. Dipeptidyl peptidase I activates neutrophil-derived serine proteases and regulates the development of acute experimental arthritis. J. Clin. Invest. 2002, 109, 363-371, doi:10.1172/JCl13462.

73. Hao, L.; Zhu, G.; Lu, Y.; Wang, M.; Jules, J.; Zhou, X.; Chen, W. Deficiency of cathepsin K prevents inflammation and bone erosion in rheumatoid arthritis and periodontitis and reveals its shared osteoimmune role. FEBS Lett. 2015, 589, 1331-1339, doi:10.1016/j.febslet.2015.04.008.

74. Ho, C.M.; Ho, S.L.; Jeng, Y.M.; Lai, Y.S.; Chen, Y.H.; Lu, S.C.; Chen, H.L.; Chang, P.Y.; Hu, R.H.; Lee, P.H. Accumulation of free cholesterol and oxidized low-density lipoprotein is associated with portal inflammation and fibrosis in nonalcoholic fatty liver disease. J. Inflamm (Lond) 2019, 16, 7, doi:10.1186/s12950-019-0211-5.

75. Kos, J.; Jevnikar, Z.; Obermajer, N. The role of cathepsin X in cell signaling. Cell Adh Migr. 2009, 3, 164-166, doi:10.4161/cam.3.2.7403.

76. Kiuchi, S.; Tomaru, U.; Ishizu, A.; Imagawa, M.; Kiuchi, T.; Iwasaki, S.; Suzuki, A.; Otsuka, N.; Deguchi, T.; Shimizu, T.; et al. Expression of cathepsins $V$ and $S$ in thymic epithelial tumors. Hum. Pathol. 2017, 60, 66-74, doi:10.1016/j.humpath.2016.09.027. 
77. Wex, T.; Buhling, F.; Wex, H.; Gunther, D.; Malfertheiner, P.; Weber, E.; Bromme, D. Human cathepsin W, a cysteine protease predominantly expressed in NK cells, is mainly localized in the endoplasmic reticulum. J. Immunol. 2001, 167, 2172-2178, doi:10.4049/jimmunol.167.4.2172.

78. Santamaria, I.; Velasco, G.; Pendas, A.M.; Fueyo, A.; Lopez-Otin, C. Cathepsin Z, a novel human cysteine proteinase with a short propeptide domain and a unique chromosomal location. J. Biol. Chem. 1998, 273, 16816-16823, doi:10.1074/jbc.273.27.16816.

79. Carlsson, S.R.; Roth, J.; Piller, F.; Fukuda, M. Isolation and Characterization of Human Lysosomal MembraneGlycoproteins, H-Lamp-1 and H-Lamp-2-Major Sialoglycoproteins Carrying Polylactosaminoglycan. J. Biol. Chem. 1988, 263, 18911-18919.

80. Boya, P.; Kroemer, G. Lysosomal membrane permeabilization in cell death. Oncogene 2008, 27, 6434-6451, doi:10.1038/onc.2008.310.

81. Kurz, T.; Terman, A.; Gustafsson, B.; Brunk, U.T. Lysosomes in iron metabolism, ageing and apoptosis. Histochem. Cell Biol. 2008, 129, 389-406, doi:10.1007/s00418-008-0394-y.

82. Kowanko, I.C.; Ferrante, A. Stimulation of neutrophil respiratory burst and lysosomal enzyme release by human interferon-gamma. Immunology 1987, 62, 149-151.

83. Hoppe, G.; O'Neil, J.; Hoff, H.F.; Sears, J. Products of lipid peroxidation induce missorting of the principal lysosomal protease in retinal pigment epithelium. Biochim. Biophys. Acta 2004, 1689, 33-41, doi:10.1016/j.bbadis.2004.01.004.

84. Yuan, X.M.; Li, W.; Olsson, A.G.; Brunk, U.T. The toxicity to macrophages of oxidized low-density lipoprotein is mediated through lysosomal damage. Atherosclerosis 1997, 133, 153-161, doi:10.1016/s0021-9150(97)00094-4.

85. Turrens, J.F. Mitochondrial formation of reactive oxygen species. J. Physiol. 2003, 552, 335-344, doi:10.1113/jphysiol.2003.049478.

86. Aits, S.; Jaattela, M. Lysosomal cell death at a glance. J. Cell Sci. 2013, 126, 1905-1912, doi:10.1242/jcs.091181.

87. Miller, D.K.; Griffiths, E.; Lenard, J.; Firestone, R.A. Cell killing by lysosomotropic detergents. J. Cell Biol. 1983, 97, 1841-1851, doi:10.1083/jcb.97.6.1841.

88. David, W.Deamera, J. Permeability of lipid bilayers to water and ionic solutes. In Chemistry and Physics of Lipids, Elsevier Scientific Publishers Ireland Ltd.: 1986; Vol. 40, pp. 167-188.

89. Deng, D.; Jiang, N.; Hao, S.J.; Sun, H.; Zhang, G.J. Loss of membrane cholesterol influences lysosomal permeability to potassium ions and protons. Biochim. Biophys. Acta 2009, 1788, 470-476, doi:10.1016/j.bbamem.2008.11.018.

90. Yi, Y.P.; Wang, X.; Zhang, G.; Fu, T.S.; Zhang, G.J. Phosphatidic acid osmotically destabilizes lysosomes through increased permeability to K+ and H+. Gen. Physiol. Biophys. 2006, 25, 149-160.

91. Goulet, B.; Baruch, A.; Moon, N.S.; Poirier, M.; Sansregret, L.L.; Erickson, A.; Bogyo, M.; Nepveu, A. A cathepsin L isoform that is devoid of a signal peptide localizes to the nucleus in S phase and processes the CDP/Cux transcription factor. Mol. Cell 2004, 14, 207-219, doi:Doi 10.1016/S1097-2765(04)00209-6.

92. Muntener, K.; Zwicky, R.; Csucs, G.; Rohrer, J.; Baici, A. Exon skipping of cathepsin B: Mitochondrial targeting of a Iysosomal peptidase provokes cell death. J. Biol. Chem. 2004, 279, 41012-41017, doi:10.1074/jbc.M405333200.

93. Bestvater, F.; Dallner, C.; Spiess, E. The C-terminal subunit of artificially truncated human cathepsin B mediates its nuclear targeting and contributes to cell viability. BMC Cell Biol. 2005, 6, 16, doi:10.1186/1471-2121-6-16.

94. Elmore, S. Apoptosis: A review of programmed cell death. Toxicol. Pathol. 2007, 35, 495-516, doi:10.1080/01926230701320337.

95. Gupta, S. Molecular steps of death receptor and mitochondrial pathways of apoptosis. Life Sci. 2001, 69, 2957-2964, doi:10.1016/s0024-3205(01)01404-7.

96. Chwieralski, C.E.; Welte, T.; Buhling, F. Cathepsin-regulated apoptosis. Apoptosis 2006, 11, 143-149, doi:10.1007/s10495-006-3486-y.

97. Droga-Mazovec, G.; Bojic, L.; Petelin, A.; Ivanova, S.; Romih, R.; Repnik, U.; Salvesen, G.S.; Stoka, V.; Turk, V.; Turk, B. Cysteine cathepsins trigger caspase-dependent cell death through cleavage of Bid and antiapoptotic Bcl-2 homologues. J. Biol. Chem. 2008, 283, 19140-19150, doi:10.1074/jbc.M802513200.

98. Conus, S.; Perozzo, R.; Reinheckel, T.; Peters, C.; Scapozza, L.; Yousefi, S.; Simon, H.U. Caspase-8 is activated by cathepsin D initiating neutrophil apoptosis during the resolution of inflammation. J. Exp. Med. 2008, 205, 685-698, doi:10.1084/jem.20072152.

99. Bidere, N.; Lorenzo, H.K.; Carmona, S.; Laforge, M.; Harper, F.; Dumont, C.; Senik, A. Cathepsin D triggers Bax activation, resulting in selective apoptosis-inducing factor (AIF) relocation in T lymphocytes entering the early commitment phase to apoptosis. J. Biol. Chem. 2003, 278, 31401-31411, doi:10.1074/jbc.M301911200.

100. Johansson, A.C.; Steen, H.; Ollinger, K.; Roberg, K. Cathepsin D mediates cytochrome c release and caspase activation in human fibroblast apoptosis induced by staurosporine. Cell Death Differ. 2003, 10, 1253-1259, doi:10.1038/sj.cdd.4401290.

101. McComb, S.; Shutinoski, B.; Thurston, S.; Cessford, E.; Kumar, K.; Sad, S. Cathepsins Limit Macrophage Necroptosis through Cleavage of Rip1 Kinase. J. Immunol. 2014, 192, 5671-5678, doi:10.4049/jimmunol.1303380.

102. Kelley, N.; Jeltema, D.; Duan, Y.; He, Y. The NLRP3 Inflammasome: An Overview of Mechanisms of Activation and Regulation. Int. J. Mol. Sci. 2019, 20, doi:10.3390/ijms20133328.

103. Campden, R.I.; Zhang, Y. The role of lysosomal cysteine cathepsins in NLRP3 inflammasome activation. Arch. Biochem. Biophys. 2019, 670, 32-42, doi:10.1016/j.abb.2019.02.015.

104. Li, S.L.; Du, L.L.; Zhang, L.; Hu, Y.; Xia, W.C.; Wu, J.; Zhu, J.; Chen, L.L.; Zhu, F.Q.; Li, C.X.; et al. Cathepsin B Contributes to Autophagy-related 7 (Atg7)-induced Nod-like Receptor 3 (NLRP3)-dependent Proinflammatory Response and 
Aggravates Lipotoxicity in Rat Insulinoma Cell Line. J. Biol. Chem. 2013, 288, 30094-30104, doi:10.1074/jbc.M113.494286.

105. Niemi, K.; Teirila, L.; Lappalainen, J.; Rajamaki, K.; Baumann, M.H.; Oorni, K.; Wolff, H.; Kovanen, P.T.; Matikainen, S.; Eklund, K.K. Serum amyloid A activates the NLRP3 inflammasome via P2X7 receptor and a cathepsin B-sensitive pathway. J. Immunol. 2011, 186, 6119-6128, doi:10.4049/jimmunol.1002843.

106. Orlowski, G.M.; Colbert, J.D.; Sharma, S.; Bogyo, M.; Robertson, S.A.; Rock, K.L. Multiple Cathepsins Promote Pro-IL1beta Synthesis and NLRP3-Mediated IL-1beta Activation. J. Immunol. 2015, 195, 1685-1697, doi:10.4049/jimmunol.1500509.

107. Soond, S.M.; Kozhevnikova, M.V.; Frolova, A.S.; Savvateeva, L.V.; Plotnikov, E.Y.; Townsend, P.A.; Han, Y.P.; Zamyatnin, A.A., Jr. Lost or Forgotten: The nuclear cathepsin protein isoforms in cancer. Cancer Lett. 2019, 462, 4350, doi:10.1016/j.canlet.2019.07.020.

108. Tamhane, T.; Illukkumbura, R.; Lu, S.Y.; Maelandsmo, G.M.; Haugen, M.H.; Brix, K. Nuclear cathepsin L activity is required for cell cycle progression of colorectal carcinoma cells. Biochimie 2016, 122, 208-218, doi:10.1016/j.biochi.2015.09.003.

109. Bach, A.S.; Derocq, D.; Laurent-Matha, V.; Montcourrier, P.; Sebti, S.; Orsetti, B.; Theillet, C.; Gongora, C.; Pattingre, S.; Ibing, E.; et al. Nuclear cathepsin D enhances TRPS1 transcriptional repressor function to regulate cell cycle progression and transformation in human breast cancer cells. Oncotarget 2015, 6, 28084-28103, doi:10.18632/oncotarget.4394.

110. Zhang, X.; Zhou, Y.; Yu, X.; Huang, Q.; Fang, W.; Li, J.; Bonventre, J.V.; Sukhova, G.K.; Libby, P.; Shi, G.P. Differential Roles of Cysteinyl Cathepsins in TGF-beta Signaling and Tissue Fibrosis. iScience 2019, 19, 607-622, doi:10.1016/j.isci.2019.08.014.

111. Leist, M.; Jaattela, M. Triggering of apoptosis by cathepsins. Cell Death Differ. 2001, 8, 324-326, doi:10.1038/sj.cdd.4400859.

112. Thibeaux, S.; Siddiqi, S.; Zhelyabovska, O.; Moinuddin, F.; Masternak, M.M.; Siddiqi, S.A. Cathepsin B regulates hepatic lipid metabolism by cleaving liver fatty acid-binding protein. J. Biol. Chem. 2018, 293, 1910-1923, doi:10.1074/jbc.M117.778365.

113. Roberts, L.R.; Kurosawa, H.; Bronk, S.F.; Fesmier, P.J.; Agellon, L.B.; Leung, W.Y.; Mao, F.; Gores, G.J. Cathepsin B contributes to bile salt-induced apoptosis of rat hepatocytes. Gastroenterology 1997, 113, 1714-1726, doi:DOI 10.1053/gast.1997.v113.pm9352877.

114. Agostini, M.; Tucci, P.; Melino, G. Cell death pathology: Perspective for human diseases. Biochem. Biophys. Res. Commun. 2011, 414, 451-455, doi:10.1016/j.bbrc.2011.09.081.

115. Mattson, M.P. Neuronal life-and-death signaling, apoptosis, and neurodegenerative disorders. Antioxid. Redox Signal. 2006, 8, 1997-2006, doi:10.1089/ars.2006.8.1997.

116. Lin, Y.; Epstein, D.L.; Liton, P.B. Intralysosomal iron induces lysosomal membrane permeabilization and cathepsin Dmediated cell death in trabecular meshwork cells exposed to oxidative stress. Invest. Ophthalmol. Vis. Sci. 2010, 51, 6483-6495, doi:10.1167/iovs.10-5410.

117. Sato, Y.; Suzuki, Y.; Ito, E.; Shimazaki, S.; Ishida, M.; Yamamoto, T.; Yamamoto, H.; Toda, T.; Suzuki, M.; Suzuki, A.; et al. Identification and characterization of an increased glycoprotein in aging: Age-associated translocation of cathepsin D. Mech. Ageing Dev. 2006, 127, 771-778, doi:10.1016/j.mad.2006.07.001.

118. Jung, H.; Lee, E.Y.; Lee, S.I. Age-related changes in ultrastructural features of cathepsin B- and D-containing neurons in rat cerebral cortex. Brain Res. 1999, 844, 43-54, doi:Doi 10.1016/S0006-8993(99)01888-0.

119. Yu, J.W.; Lee, M.S. Mitochondria and the NLRP3 inflammasome: Physiological and pathological relevance. Arch. Pharm. Res. 2016, 39, 1503-1518, doi:10.1007/s12272-016-0827-4.

120. Cocchiaro, P.; De Pasquale, V.; Della Morte, R.; Tafuri, S.; Avallone, L.; Pizard, A.; Moles, A.; Pavone, L.M. The Multifaceted Role of the Lysosomal Protease Cathepsins in Kidney Disease. Front. Cell Dev. Biol. 2017, 5, 114, doi:10.3389/fcell.2017.00114.

121. Sever, S.; Altintas, M.M.; Nankoe, S.R.; Moller, C.C.; Ko, D.; Wei, C.L.; Henderson, J.; del Re, E.C.; Hsing, L.; Erickson, A.; et al. Proteolytic processing of dynamin by cytoplasmic cathepsin $\mathrm{L}$ is a mechanism for proteinuric kidney disease. J. Clin. Investig. 2007, 117, 2095-2104, doi:10.1172/Jci32022.

122. Maubach, G.; Lim, M.C.; Zhuo, L. Nuclear cathepsin F regulates activation markers in rat hepatic stellate cells. Mol. Biol. Cell 2008, 19, 4238-4248, doi:10.1091/mbc.E08-03-0291.

123. Baici, A.; Muntener, K.; Willimann, A.; Zwicky, R. Regulation of human cathepsin B by alternative mRNA splicing: Homeostasis, fatal errors and cell death. Biol. Chem. 2006, 387, 1017-1021, doi:10.1515/Bc.2006.125.

124. Olson, O.C.; Joyce, J.A. Cysteine cathepsin proteases: Regulators of cancer progression and therapeutic response. Nat. Rev. Cancer 2015, 15, 712-729, doi:10.1038/nrc4027.

125. Samie, M.A.; Xu, H. Lysosomal exocytosis and lipid storage disorders. J. Lipid Res. 2014, 55, 995-1009, doi:10.1194/jlr.R046896.

126. Guardia, C.M.; Farias, G.G.; Jia, R.; Pu, J.; Bonifacino, J.S. BORC Functions Upstream of Kinesins 1 and 3 to Coordinate Regional Movement of Lysosomes along Different Microtubule Tracks. Cell Rep. 2016, 17, 1950-1961, doi:10.1016/j.celrep.2016.10.062.

127. Rao, S.K.; Huynh, C.; Proux-Gillardeaux, V.; Galli, T.; Andrews, N.W. Identification of SNAREs involved in synaptotagmin VII-regulated lysosomal exocytosis. J. Biol. Chem. 2004, 279, 20471-20479, doi:10.1074/jbc.M400798200. 
128. Medina, D.L.; Fraldi, A.; Bouche, V.; Annunziata, F.; Mansueto, G.; Spampanato, C.; Puri, C.; Pignata, A.; Martina, J.A.; Sardiello, M.; et al. Transcriptional activation of lysosomal exocytosis promotes cellular clearance. Dev. Cell 2011, 21, 421-430, doi:10.1016/j.devcel.2011.07.016.

129. Castro-Gomes, T.; Corrotte, M.; Tam, C.; Andrews, N.W. Plasma Membrane Repair Is Regulated Extracellularly by Proteases Released from Lysosomes. Plos ONE 2016, 11, e0152583, doi:10.1371/journal.pone.0152583.

130. Olson, L.J.; Hindsgaul, O.; Dahms, N.M.; Kim, J.J. Structural insights into the mechanism of pH-dependent ligand binding and release by the cation-dependent mannose 6-phosphate receptor. J. Biol. Chem. 2008, 283, 10124-10134, doi:10.1074/jbc.M708994200.

131. Jane, D.T.; Morvay, L.; DaSilva, L.; Cavallo-Medved, D.; Sloane, B.F.; Dufresne, M.J. Cathepsin B localizes to plasma membrane caveolae of differentiating myoblasts and is secreted in an active form at physiological pH. Biol. Chem. 2006, 387, 223-234, doi:10.1515/Bc.2006.030.

132. Jordans, S.; Jenko-Kokalj, S.; Kuhl, N.M.; Tedelind, S.; Sendt, W.; Bromme, D.; Turk, D.; Brix, K. Monitoring compartment-specific substrate cleavage by cathepsins $\mathrm{B}, \mathrm{K}, \mathrm{L}$, and $\mathrm{S}$ at physiological $\mathrm{pH}$ and redox conditions. $B M C$ Biochem. 2009, 10, doi:Artn 23.10.1186/1471-2091-10-23.

133. Uhlman, A.; Folkers, K.; Liston, J.; Pancholi, H.; Hinton, A. Effects of Vacuolar H(+)-ATPase Inhibition on Activation of Cathepsin B and Cathepsin L Secreted from MDA-MB231 Breast Cancer Cells. Cancer Microenviron. 2017, 10, 49-56, doi:10.1007/s12307-017-0196-7.

134. Yang, D.Q.; Feng, S.; Chen, W.; Zhao, H.; Paulson, C.; Li, Y.P. V-ATPase subunit ATP6AP1 (Ac45) regulates osteoclast differentiation, extracellular acidification, lysosomal trafficking, and protease exocytosis in osteoclast-mediated bone resorption. J. Bone Min. Res. 2012, 27, 1695-1707, doi:10.1002/jbmr.1623.

135. Runger, T.M.; Quintanilla-Dieck, M.J.; Bhawan, J. Role of cathepsin K in the turnover of the dermal extracellular matrix during scar formation. J. Invest. Derm. 2007, 127, 293-297, doi:10.1038/sj.jid.5700535.

136. Goto, T.; Yamaza, T.; Tanaka, T. Cathepsins in the osteoclast. J. Electron. Microsc (Tokyo) 2003, 52, 551-558, doi:10.1093/jmicro/52.6.551.

137. Saftig, P.; Hunziker, E.; Everts, V.; Jones, S.; Boyde, A.; Wehmeyer, O.; Suter, A.; von Figura, K. Functions of cathepsin $\mathrm{K}$ in bone resorption. Lessons from cathepsin $\mathrm{K}$ deficient mice. Adv. Exp. Med. Biol. 2000, 477, 293-303, doi:10.1007/0-306-46826-3_32.

138. Bonnans, C.; Chou, J.; Werb, Z. Remodelling the extracellular matrix in development and disease. Nat. Rev. Mol. Cell Biol. 2014, 15, 786-801, doi:10.1038/nrm3904.

139. Fonovic, M.; Turk, B. Cysteine cathepsins and extracellular matrix degradation. Biochim. Biophys. Acta 2014, 1840, 2560-2570, doi:10.1016/j.bbagen.2014.03.017.

140. Raggatt, L.J.; Partridge, N.C. Cellular and molecular mechanisms of bone remodeling. J. Biol. Chem. 2010, 285, 2510325108, doi:10.1074/jbc.R109.041087.

141. Troen, B.R. The role of cathepsin K in normal bone resorption. Drug News Perspect. 2004, 17, 19-28, doi:10.1358/dnp.2004.17.1.829022.

142. Page, A.E.; Hayman, A.R.; Andersson, L.M.B.; Chambers, T.J.; Warburton, M.J. Degradation of Bone-Matrix Proteins by Osteoclast Cathepsins. Int. J. Biochem. 1993, 25, 545-550, doi:Doi 10.1016/0020-711x(93)90662-X.

143. Saini, M.G.; Bix, G.J. Oxygen-glucose deprivation (OGD) and interleukin-1 (IL-1) differentially modulate cathepsin B/L mediated generation of neuroprotective perlecan LG3 by neurons. Brain Res. 2012, 1438, 65-74, doi:10.1016/j.brainres.2011.12.027.

144. Roberts, J.; Kahle, M.P.; Bix, G.J. Perlecan and the blood-brain barrier: Beneficial proteolysis? Front. Pharm. 2012, 3, 155, doi:10.3389/fphar.2012.00155.

145. Buth, H.; Luigi Buttigieg, P.; Ostafe, R.; Rehders, M.; Dannenmann, S.R.; Schaschke, N.; Stark, H.J.; Boukamp, P.; Brix, K. Cathepsin B is essential for regeneration of scratch-wounded normal human epidermal keratinocytes. Eur. J. Cell Biol. 2007, 86, 747-761, doi:10.1016/j.ejcb.2007.03.009.

146. Obermajer, N.; Repnik, U.; Jevnikar, Z.; Turk, B.; Kreft, M.; Kos, J. Cysteine protease cathepsin X modulates immune response via activation of beta2 integrins. Immunology 2008, 124, 76-88, doi:10.1111/j.1365-2567.2007.02740.x.

147. Hook, V.; Yasothornsrikul, S.; Greenbaum, D.; Medzihradszky, K.F.; Troutner, K.; Toneff, T.; Bundey, R.; Logrinova, A.; Reinheckel, T.; Peters, C.; et al. Cathepsin L and Arg/Lys aminopeptidase: A distinct prohormone processing pathway for the biosynthesis of peptide neurotransmitters and hormones. Biol. Chem. 2004, 385, 473-480, doi:10.1515/BC.2004.055.

148. Funkelstein, L.; Lu, W.D.; Koch, B.; Mosier, C.; Toneff, T.; Taupenot, L.; O'Connor, D.T.; Reinheckel, T.; Peters, C.; Hook, V. Human cathepsin $\mathrm{V}$ protease participates in production of enkephalin and NPY neuropeptide neurotransmitters. J. Biol. Chem. 2012, 287, 15232-15241, doi:10.1074/jbc.M111.310607.

149. Hwang, S.R.; Garza, C.; Mosier, C.; Toneff, T.; Wunderlich, E.; Goldsmith, P.; Hook, V. Cathepsin L expression is directed to secretory vesicles for enkephalin neuropeptide biosynthesis and secretion. J. Biol. Chem. 2007, 282, 9556-9563, doi:10.1074/jbc.M605510200.

150. Yasothornsrikul, S.; Greenbaum, D.; Medzihradszky, K.F.; Toneff, T.; Bundey, R.; Miller, R.; Schilling, B.; Petermann, I.; Dehnert, J.; Logvinova, A.; et al. Cathepsin L in secretory vesicles functions as a prohormone-processing enzyme for production of the enkephalin peptide neurotransmitter. Proc. Natl. Acad. Sci. USA 2003, 100, 9590-9595, doi:10.1073/pnas.1531542100. 
151. Yan, D.; Wang, H.W.; Bowman, R.L.; Joyce, J.A. STAT3 and STAT6 Signaling Pathways Synergize to Promote Cathepsin Secretion from Macrophages via IRE1alpha Activation. Cell Rep. 2016, 16, 2914-2927, doi:10.1016/j.celrep.2016.08.035.

152. Sloane, B.F.; Yan, S.; Podgorski, I.; Linebaugh, B.E.; Cher, M.L.; Mai, J.; Cavallo-Medved, D.; Sameni, M.; Dosescu, J.; Moin, K. Cathepsin B and tumor proteolysis: Contribution of the tumor microenvironment. Semin Cancer Biol 2005, 15, 149-157, doi:10.1016/j.semcancer.2004.08.001.

153. Hashimoto, Y.; Kondo, C.; Katunuma, N. An Active 32-kDa Cathepsin L Is Secreted Directly from HT 1080 Fibrosarcoma Cells and Not via Lysosomal Exocytosis. Plos ONE 2015, 10, e0145067, doi:10.1371/journal.pone.0145067.

154. Nicotra, G.; Castino, R.; Follo, C.; Peracchio, C.; Valente, G.; Isidoro, C. The dilemma: Does tissue expression of cathepsin $D$ reflect tumor malignancy? The question: Does the assay truly mirror cathepsin $D$ mis-function in the tumor? Cancer Biomark 2010, 7, 47-64, doi:10.3233/CBM-2010-0143.

155. Gocheva, V.; Zeng, W.; Ke, D.; Klimstra, D.; Reinheckel, T.; Peters, C.; Hanahan, D.; Joyce, J.A. Distinct roles for cysteine cathepsin genes in multistage tumorigenesis. Genes Dev. 2006, 20, 543-556, doi:10.1101/gad.1407406.

156. Lkhider, M.; Castino, R.; Bouguyon, E.; Isidoro, C.; Ollivier-Bousquet, M. Cathepsin D released by lactating rat mammary epithelial cells is involved in prolactin cleavage under physiological conditions. J. Cell Sci. 2004, 117, 51555164, doi:10.1242/jcs.01396.

157. Erdmann, S.; Ricken, A.; Merkwitz, C.; Struman, I.; Castino, R.; Hummitzsch, K.; Gaunitz, F.; Isidoro, C.; Martial, J.; Spanel-Borowski, K. The expression of prolactin and its cathepsin D-mediated cleavage in the bovine corpus luteum vary with the estrous cycle. Am. J. Physiol. Endocrinol. Metab. 2007, 293, E1365-1377, doi:10.1152/ajpendo.00280.2007.

158. Sobotic, B.; Vizovisek, M.; Vidmar, R.; Van Damme, P.; Gocheva, V.; Joyce, J.A.; Gevaert, K.; Turk, V.; Turk, B.; Fonovic, M. Proteomic Identification of Cysteine Cathepsin Substrates Shed from the Surface of Cancer Cells. Mol. Cell Proteom. 2015, 14, 2213-2228, doi:10.1074/mcp.M114.044628.

159. Mizunoe, Y.; Kobayashi, M.; Tagawa, R.; Nakagawa, Y.; Shimano, H.; Higami, Y. Association between Lysosomal Dysfunction and Obesity-Related Pathology: A Key Knowledge to Prevent Metabolic Syndrome. Int. J. Mol. Sci. 2019, 20, doi:10.3390/ijms20153688.

160. Bourne, L.C.; Lamb, D.J.; Collis, C.S.; O’Brien, M.; Leake, D.S.; Rice-Evans, C. Non-oxidative modification of low density lipoprotein by ruptured myocytes. FEBS Lett. 1997, 414, 576-580, doi:10.1016/s0014-5793(97)01075-2.

161. Benes, P.; Vetvicka, V.; Fusek, M. Cathepsin D--many functions of one aspartic protease. Crit. Rev. Oncol. Hematol. 2008, 68, 12-28, doi:10.1016/j.critrevonc.2008.02.008.

162. Khurana, P.; Yadati, T.; Goyal, S.; Dolas, A.; Houben, T.; Oligschlaeger, Y.; Agarwal, A.K.; Kulkarni, A.; Shiri-Sverdlov, R. Inhibiting Extracellular Cathepsin D Reduces Hepatic Steatosis in Sprague(-)Dawley Rats (dagger). Biomolecules 2019, 9, doi:10.3390/biom9050171.

163. Zhao, C.F.; Herrington, D.M. The function of cathepsins B, D, and X in atherosclerosis. Am. J. Cardiovasc. Dis. 2016, 6, 163-170.

164. Wiener, J.J.; Sun, S.; Thurmond, R.L. Recent advances in the design of cathepsin S inhibitors. Curr. Top. Med. Chem. 2010, 10, 717-732, doi:10.2174/156802610791113432.

165. Maciewicz, R.A.; Wotton, S.F. Degradation of cartilage matrix components by the cysteine proteinases, cathepsins B and L. Biomed. Biochim. Acta 1991, 50, 561-564.

166. Buck, M.R.; Karustis, D.G.; Day, N.A.; Honn, K.V.; Sloane, B.F. Degradation of extracellular-matrix proteins by human cathepsin B from normal and tumour tissues. Biochem. J. 1992, 282 (Pt. 1), 273-278, doi:10.1042/bj2820273.

167. Ishidoh, K.; Kominami, E. Procathepsin L degrades extracellular matrix proteins in the presence of glycosaminoglycans in vitro. Biochem. Biophys. Res. Commun. 1995, 217, 624-631, doi:10.1006/bbrc.1995.2820.

168. Wang, B.; Sun, J.; Kitamoto, S.; Yang, M.; Grubb, A.; Chapman, H.A.; Kalluri, R.; Shi, G.P. Cathepsin S controls angiogenesis and tumor growth via matrix-derived angiogenic factors. J. Biol. Chem. 2006, 281, 6020-6029, doi:10.1074/jbc.M509134200.

169. Son, E.D.; Kim, H.; Choi, H.; Lee, S.H.; Lee, J.Y.; Kim, S.; Closs, B.; Lee, S.; Chung, J.H.; Hwang, J.S. Cathepsin G increases MMP expression in normal human fibroblasts through fibronectin fragmentation, and induces the conversion of proMMP-1 to active MMP-1. J. Derm. Sci. 2009, 53, 150-152, doi:10.1016/j.jdermsci.2008.08.006.

170. Mai, J.; Sameni, M.; Mikkelsen, T.; Sloane, B.F. Degradation of extracellular matrix protein tenascin-C by cathepsin B: An interaction involved in the progression of gliomas. Biol. Chem. 2002, 383, 1407-1413, doi:10.1515/BC.2002.159.

171. Mort, J.S.; Magny, M.C.; Lee, E.R. Cathepsin B: An alternative protease for the generation of an aggrecan 'metalloproteinase' cleavage neoepitope. Biochem. J. 1998, 335 (Pt. 3), 491-494, doi:10.1042/bj3350491.

172. Garcia, M.; Platet, N.; Liaudet, E.; Laurent, V.; Derocq, D.; Brouillet, J.P.; Rochefort, H. Biological and clinical significance of cathepsin D in breast cancer metastasis. Stem Cells 1996, 14, 642-650, doi:10.1002/stem.140642.

173. Du, X.; Chen, N.L.; Wong, A.; Craik, C.S.; Bromme, D. Elastin degradation by cathepsin V requires two exosites. J. Biol. Chem. 2013, 288, 34871-34881, doi:10.1074/jbc.M113.510008.

174. Staudt, N.D.; Aicher, W.K.; Kalbacher, H.; Stevanovic, S.; Carmona, A.K.; Bogyo, M.; Klein, G. Cathepsin X is secreted by human osteoblasts, digests CXCL-12 and impairs adhesion of hematopoietic stem and progenitor cells to osteoblasts. Haematologica 2010, 95, 1452-1460, doi:10.3324/haematol.2009.018671.

175. Ondr, J.K.; Pham, C.T. Characterization of murine cathepsin W and its role in cell-mediated cytotoxicity. J. Biol. Chem. 2004, 279, 27525-27533, doi:10.1074/jbc.M400304200. 
176. Fonovic, M.; Turk, B. Cysteine cathepsins and their potential in clinical therapy and biomarker discovery. Proteom. Clin. Appl. 2014, 8, 416-426, doi:10.1002/prca.201300085.

177. Joyce, J.A.; Baruch, A.; Chehade, K.; Meyer-Morse, N.; Giraudo, E.; Tsai, F.Y.; Greenbaum, D.C.; Hager, J.H.; Bogyo, M.; Hanahan, D. Cathepsin cysteine proteases are effectors of invasive growth and angiogenesis during multistage tumorigenesis. Cancer Cell 2004, 5, 443-453, doi:10.1016/s1535-6108(04)00111-4.

178. Gabrijelcic, D.; Svetic, B.; Spaic, D.; Skrk, J.; Budihna, M.; Dolenc, I.; Popovic, T.; Cotic, V.; Turk, V. Cathepsins B, H and L in human breast carcinoma. Eur. J. Clin. Chem. Clin. Biochem. 1992, 30, 69-74.

179. Hashimoto, Y.; Kondo, C.; Kojima, T.; Nagata, H.; Moriyama, A.; Hayakawa, T.; Katunuma, N. Significance of 32-kDa cathepsin L secreted from cancer cells. Cancer Biother. Radiopharm. 2006, 21, 217-224, doi:10.1089/cbr.2006.21.217.

180. Singh, N.; Das, P.; Gupta, S.; Sachdev, V.; Srivasatava, S.; Datta Gupta, S.; Pandey, R.M.; Sahni, P.; Chauhan, S.S.; Saraya, A. Plasma cathepsin L: A prognostic marker for pancreatic cancer. World J. Gastroenterol. 2014, 20, 17532-17540, doi:10.3748/wjg.v20.i46.17532.

181. Ruan, J.; Zheng, H.; Fu, W.; Zhao, P.; Su, N.; Luo, R. Increased expression of cathepsin L: A novel independent prognostic marker of worse outcome in hepatocellular carcinoma patients. Plos ONE 2014, 9, e112136, doi:10.1371/journal.pone.0112136.

182. Schweiger, A.; Staib, A.; Werle, B.; Krasovec, M.; Lah, T.T.; Ebert, W.; Turk, V.; Kos, J. Cysteine proteinase cathepsin H in tumours and sera of lung cancer patients: Relation to prognosis and cigarette smoking. Br. J. Cancer 2000, 82, 782788, doi:DOI 10.1054/bjoc.1999.0999.

183. Kos, J.; Stabuc, B.; Schweiger, A.; Krasovec, M.; Cimerman, N.; KopitarJerala, N.; Vrhovec, I. Cathepsins B, H, and L and their inhibitors stefin A and cystatin C in sera of melanoma patients. Clin. Cancer Res. 1997, 3, 1815-1822.

184. Schweiger, A.; Christensen, I.J.; Nielsen, H.J.; Sorensen, S.; Brunner, N.; Kos, J. Serum cathepsin H as a potential prognostic marker in patients with colorectal cancer. Int. J. Biol. Markers 2004, 19, 289-294, doi:10.5301/jbm.2008.4105.

185. Kos, J.; Werle, B.; Lah, T.; Brunner, N. Cysteine proteinases and their inhibitors in extracellular fluids: Markers for diagnosis and prognosis in cancer. Int. J. Biol. Markers 2000, 15, 84-89.

186. Wu, H.; Du, Q.; Dai, Q.; Ge, J.; Cheng, X. Cysteine Protease Cathepsins in Atherosclerotic Cardiovascular Diseases. J. Atheroscler. Thromb. 2018, 25, 111-123, doi:10.5551/jat.RV17016.

187. Walenbergh, S.M.; Houben, T.; Rensen, S.S.; Bieghs, V.; Hendrikx, T.; van Gorp, P.J.; Oligschlaeger, Y.; Jeurissen, M.L.; Gijbels, M.J.; Buurman, W.A.; et al. Plasma cathepsin D correlates with histological classifications of fatty liver disease in adults and responds to intervention. Sci. Rep. 2016, 6, 38278, doi:10.1038/srep38278.

188. Liu, L.; Chen, B.; Zhang, X.; Tan, L.; Wang, D.W. Increased Cathepsin D Correlates with Clinical Parameters in Newly Diagnosed Type 2 Diabetes. Dis. Markers 2017, 2017, 5286408, doi:10.1155/2017/5286408.

189. Ding, L.L.; Goossens, G.H.; Oligschlaeger, Y.; Houben, T.; Blaak, E.E.; Shiri-Sverdlov, R. Plasma cathepsin D activity is negatively associated with hepatic insulin sensitivity in overweight and obese humans. Diabetologia 2019, 10.1007/s00125-019-05025-2, doi:10.1007/s00125-019-05025-2.

190. Dera, A.A.; Ranganath, L.; Barraclough, R.; Vinjamuri, S.; Hamill, S.; Barraclough, D.L. Cathepsin Z as a novel potential biomarker for osteoporosis. Sci. Rep. 2019, 9, doi:ARTN 9752.10.1038/s41598-019-46068-0.

191. Wang, L.; Hu, Y.Q.; Zhao, Z.J.; Zhang, H.Y.; Gao, B.; Lu, W.G.; Xu, X.L.; Lin, X.S.; Wang, J.P.; Jie, Q.; et al. Screening and validation of serum protein biomarkers for early postmenopausal osteoporosis diagnosis. Mol. Med. Rep. 2017, 16, 8427-8433, doi:10.3892/mmr.2017.7620.

192. Kramer, L.; Turk, D.; Turk, B. The Future of Cysteine Cathepsins in Disease Management. Trends Pharm. Sci. 2017, 38, 873-898, doi:10.1016/j.tips.2017.06.003.

193. Marques, A.R.A.; Di Spiezio, A.; Thiessen, N.; Schmidt, L.; Grotzinger, J.; Lullmann-Rauch, R.; Damme, M.; Storck, S.E.; Pietrzik, C.U.; Fogh, J.; et al. Enzyme replacement therapy with recombinant pro-CTSD (cathepsin D) corrects defective proteolysis and autophagy in neuronal ceroid lipofuscinosis. Autophagy 2020, 16, 811-825, doi:10.1080/15548627.2019.1637200.

194. Desnick, R.J.; Schuchman, E.H. Enzyme replacement therapy for lysosomal diseases: Lessons from 20 years of experience and remaining challenges. Annu. Rev. Genom. Hum. Genet. 2012, 13, 307-335, doi:10.1146/annurevgenom-090711-163739.

195. Khaket, T.P.; Kwon, T.K.; Kang, S.C. Cathepsins: Potent regulators in carcinogenesis. Pharm. Ther.2019, 198, 1-19, doi:10.1016/j.pharmthera.2019.02.003.

196. Verbovsek, U.; Van Noorden, C.J.; Lah, T.T. Complexity of cancer protease biology: Cathepsin K expression and function in cancer progression. Semin. Cancer Biol. 2015, 35, 71-84, doi:10.1016/j.semcancer.2015.08.010.

197. Mullard, A. Merck \&Co. drops osteoporosis drug odanacatib. Nat. Rev. Drug Discov. 2016, 15, 669, doi:10.1038/nrd.2016.207.

198. Panwar, P.; Soe, K.; Guido, R.V.; Bueno, R.V.; Delaisse, J.M.; Bromme, D. A novel approach to inhibit bone resorption: Exosite inhibitors against cathepsin K. Br. J. Pharm. 2016, 173, 396-410, doi:10.1111/bph.13383.

199. Panwar, P.; Xue, L.M.; Soe, K.; Srivastava, K.; Law, S.; Delaisse, J.M.; Bromme, D. An Ectosteric Inhibitor of Cathepsin K Inhibits Bone Resorption in Ovariectomized Mice (vol 32, pg 2415, 2017). J. Bone Miner. Res. 2019, 34, 777-778, doi:10.1002/jbmr.3687. 


\section{Chapter 2}

200. Burden, R.E.; Gormley, J.A.; Jaquin, T.J.; Small, D.M.; Quinn, D.J.; Hegarty, S.M.; Ward, C.; Walker, B.; Johnston, J.A.; Olwill, S.A.; et al. Antibody-mediated inhibition of cathepsin S blocks colorectal tumor invasion and angiogenesis. Clin. Cancer Res. 2009, 15, 6042-6051, doi:10.1158/1078-0432.CCR-09-1262.

201. Burden, R.E.; Gormley, J.A.; Kuehn, D.; Ward, C.; Kwok, H.F.; Gazdoiu, M.; McClurg, A.; Jaquin, T.J.; Johnston, J.A.; Scott, C.J.; et al. Inhibition of Cathepsin S by Fsn0503 enhances the efficacy of chemotherapy in colorectal carcinomas. Biochimie 2012, 94, 487-493, doi:10.1016/j.biochi.2011.08.017.

202. Mirkovic, B.; Markelc, B.; Butinar, M.; Mitrovic, A.; Sosic, I.; Gobec, S.; Vasiljeva, O.; Turk, B.; Cemazar, M.; Sersa, G.; et al. Nitroxoline impairs tumor progression in vitro and in vivo by regulating cathepsin B activity. Oncotarget. 2015, 6, 19027-19042, doi:10.18632/oncotarget.3699.

203. Mikhaylov, G.; Klimpel, D.; Schaschke, N.; Mikac, U.; Vizovisek, M.; Fonovic, M.; Turk, V.; Turk, B.; Vasiljeva, O. Selective targeting of tumor and stromal cells by a nanocarrier system displaying lipidated cathepsin $B$ inhibitor. Angew. Chem. In.T Ed. Eng/ 2014, 53, 10077-10081, doi:10.1002/anie.201402305. 


\section{Chapter 3}

\section{Inhibiting extracellular cathepsin D reduces hepatic steatosis in Sprague-Dawley rats}

Princy Khurana\#, Tulasi Yadati\#, Sandeep Goyal, Atul Dolas, Tom Houben, Yvonne Oligschlaeger, Anil K. Agarwal, Aditya Kulkarni, Ronit Shiri-Sverdlov \#Authors equally contributed Biomolecules. 2019 May; 9(5): 171. 


\begin{abstract}
Dietary and lifestyle changes are leading to an increased occurrence of non-alcoholic fatty liver disease (NAFLD). Using a hyperlipidemic murine model for non-alcoholic steatohepatitis (NASH), we have previously demonstrated that the lysosomal protease cathepsin D (CTSD) is involved with lipid dysregulation and inflammation. However, despite identifying CTSD as a major player in NAFLD pathogenesis, the specific role of extracellular CTSD in NAFLD has not yet been investigated. Given that inhibition of intracellular CTSD is highly unfavorable due to its fundamental physiological function, we here investigated the impact of a highly specific and potent small-molecule inhibitor of extracellular CTSD (CTD-002) in the context of NAFLD. Treatment of bone marrow-derived macrophages with CTD-002, and incubation of hepatic HepG2 cells with a conditioned medium derived from CTD-002-treated macrophages, resulted in reduced levels of inflammation and improved cholesterol metabolism. Treatment with CTD-002 improved hepatic steatosis in high fat diet-fed rats. Additionally, plasma levels of insulin and hepatic transaminases were significantly reduced upon CTD-002 administration. Collectively, our findings demonstrate for the first time that modulation of extracellular CTSD can serve as a novel therapeutic modality for NAFLD.
\end{abstract}




\section{Introduction}

Non-alcoholic fatty liver disease (NAFLD) is one of the most common liver diseases in the world with an estimated prevalence of $25 \%$ in the adult population [1]. Histologically, NAFLD ranges from steatosis, which is the benign accumulation of macro-vesicular and micro-vesicular lipid droplets in hepatocytes, to the more severe form of NAFLD referred to as non-alcoholic steatohepatitis (NASH). NASH is a combination of hepatic steatosis and inflammation with or without fibrosis [2], which can progress further to severe conditions such as liver cirrhosis and liver failure [3,4]. Due to the lack of mechanistic insights into the pathogenesis of NAFLD [5], clinically approved treatment options do not yet exist.

NAFLD is characterized by an impaired hepatic lipid profile, which is in turn characterized by dysregulation of cholesterol and triglyceride metabolism [6]. It has been well-established that lysosomes, in particular their lysosomal proteases such as cathepsins, play a crucial role in maintaining metabolic processes such as lipid homeostasis [7-10]. Specifically, it has been shown that changes in the expression of the lysosomal enzyme cathepsin D (CTSD) are associated with differences in cholesterol metabolism [11-13]. Relevantly, though CTSD is mainly functional within the intracellular, acidic milieu of lysosomes, in response to certain physiological and pathological conditions, CTSD is secreted extracellularly [11]. Strikingly, and similar to its intracellular fraction, it has been shown that CTSD is also proteolytically active upon secretion into the extracellular space $[14,15]$. Indeed, given that CTSD retains its catalytic activity at neutral pH [16], these data indicate a potential role for extracellular CTSD in lipid-related disorders such as NAFLD $[17,18]$.

Recently, we have demonstrated that targeting the proteolytic activity of CTSD using pepstatin-A (Pep-A), a known inhibitor of CTSD, remarkably reduced steatohepatitis in a hyperlipidemic mouse model, thereby pointing towards a role for CTSD activity in NAFLD [19]. However, the observed effects in this study were the result of a targeted inhibition of intracellular and extracellular CTSD. Moreover, Pep-A is a potent but relatively unspecific inhibitor of aspartic proteases [20]. Hence, in the current study, we developed a highly specific and potent small-molecule inhibitor of extracellular CTSD, referred to as CTD-002 (IC50 = 28 nM) in order to investigate its efficacy in treating NAFLD.

We hypothesized that specific inhibition of extracellular CTSD activity has a therapeutic benefit for NAFLD. In order to test our hypothesis, Sprague-Dawley (SD) rats fed a high-fat diet (HFD), known as the Lieber-Decarli diet, were used as an in vivo NAFLD model [21] and were injected with the CTD002. In addition, wild-type ( $\mathrm{Wt}$ ) bone marrow-derived macrophages (BMDMs) treated with CTD-002 were used as an in vitro model to assess the ability of CTD-002 to reduce inflammation. Moreover, to investigate whether the CTD-002 inhibitor influences the inflammatory crosstalk between hepatic immune and parenchymal cells, a conditioned medium derived from BMDMs was transferred to HepG2 liver cells. Treating BMDMs with CTD-002 resulted in a decrease in inflammation and improvement in lipid metabolism. In addition, incubating hepatic HepG2 cells with the conditioned medium derived from CTD-002-treated BMDMs showed a decreasing trend in inflammation compared to control cells. Sprague-Dawley rats fed an HFD showed decreased hepatic steatosis and inflammation upon treatment with CTD-002. Altogether, these data suggest that targeting extracellular CTSD potentially represents a novel and effective therapeutic strategy for NAFLD. 


\section{Materials and methods}

\subsection{Design and development of CTD-002}

CTD-002 was designed using the Schrodinger Small Molecule Drug Design Suite and medicinal chemistry efforts. In this process, a library of $\sim 500,000$ commercially available compounds were screened in silico using the pharmacophore modelling $[22,23]$ in PHASE module and docking the top 10,000 molecules via the GLIDE XP [24,25] module of the Schrodinger suite (v7.2, Schrödinger, LLC). This resulted in the identification of a set of 100 molecules with diverse chemical structures. These molecules were then further screened against CTSD in a cell-free assay to identify those compounds that had micromolar or higher potency. The compounds with the lowest permeability and efflux were selected as exerting most of its effects extracellularly. Finally, these compounds were further refined and modified to create a library of compounds with nanomolar efficacy against CTSD, with CTD-002 being the most potent small-molecule inhibitor, showing a dose-dependent inhibition of CTSD activity (Figure S1). While the compound may have minor effects of intracellular CTSD, toxicity studies in Sprague-Dawley rats and BALB/c mice, a widely used mouse strain, showed no toxic reactions at the therapeutic dose of $50 \mathrm{mg} / \mathrm{kg}$, which was injected twice a week for six weeks.

\subsection{Isolation and culturing of bone marrow-derived macrophages}

Tibiae and femurs of wild type mice belonging to $\mathrm{C} 75 \mathrm{BI} / 6$ strain were broken and used for harvesting bone marrow-derived macrophages (BMDMs). The culture medium in which the BMDMs were cultured was an RPMI medium (GIBCO Invitrogen, Breda, the Netherlands) supplemented with $10 \%$ heat-inactivated fetal calf serum (Bodinco B.V. Alkmaar, the Netherlands), penicillin $(100 \mathrm{U} / \mathrm{mL}$ ), streptomycin $(100 \mu \mathrm{g} / \mathrm{mL}$ ) and L-glutamine $2 \mathrm{mM}$ (all derived from GIBCO Invitrogen, Breda, the Netherlands). To differentiate between monocytes and macrophages, the culture medium was also supplemented with 20\% L929-conditioned medium (LCM) for 9 days. At day 10, BMDMs were cultured in a 24-well plate at 350,000 cells per well. Next, the BMDMs' culture medium was enriched with oxidized low-density lipoprotein (oxLDL) for 24 h (25 $\mathrm{gg} / \mathrm{mL}$; Alfa Aesar: J65591, Wardhill, MA, USA). Then, the macrophages were treated with CTD-002 (Aten Porus Lifesciences Pvt Ltd., India) or with carrier-control dimethyl sulphoxide (DMSO) for $4 \mathrm{~h}$. In order to increase the effects on inflammation, cells were washed and further stimulated with lipopolysaccharide (LPS; $100 \mathrm{ng} / \mathrm{mL}$ ) for $4 \mathrm{~h}$.

To explore the possible effects of secreted CTSD on neighboring cells, hepatic HepG2 (ATCC HB-8065) cells were incubated with the conditioned medium from CTD-002-treated BMDMs, or control-treated BMDMs, for $4 \mathrm{~h}$. Subsequently, cells were washed and stimulated with LPS (100 ng/mL) for $4 \mathrm{~h}$, after which the supernatant was collected for protein measurements and cells were lysed for mRNA expression analyses.

\subsection{Rats, diet and intervention}

Animal experiments were conducted at Theralndx Lifesciences Private Limited, following all ethical practices as formulated in the guidelines for animal care and approved by the Institutional Animals 
Ethics Committee (IAEC; protocol no. IAEC/05/2017/064), India. SD rats were given free access to food and water and were housed under standard conditions. Following an acclimatization period of seven days, rats were fed with either low-fat (LFD) or HFD for three weeks. The composition of the diets is listed in Table S1. To test its therapeutic efficacy, CTD-002 was administered twice a week intraperitoneally (dose $50 \mathrm{mg} / \mathrm{kg}$ ) for three weeks. The experimental conditions are also shown in Figure S2. After three weeks, the animals were sacrificed using $\mathrm{CO} 2$ anesthesia. Following blood collection, the rats were dissected, liver tissues were excised, weighed and cut into small pieces weighing 100 to $200 \mathrm{mg}$. These liver tissues were frozen immediately in liquid nitrogen and stored at $-80{ }^{\circ} \mathrm{C}$ for further analyses. In addition, liver samples were also fixed in $4 \%$ formaldehyde for histologic examination. Blood was collected by cardiac puncture followed by termination, centrifuged and plasma was harvested for biochemical analyses.

\subsection{Histological analyses}

Formalin-fixed liver samples were stained with Hematoxylin-Eosin (H\&E) in order to score fat vesicles as minimal, mild, moderate and severe steatosis. Lobular inflammatory activity was scored as follows: 1) focal collections of mononuclear inflammatory cells; 2) diffuse infiltrates of mononuclear inflammatory cells and 3 ) focal collections of polymorphonuclear cells in addition to mononuclear cell infiltrates. In addition to H\&E, formalin-fixed liver samples stained with Chromotrope Aniline Blue and Sirius Red were quantified for total hepatic fat percentage by means of digital image analysis in ImageJ.

\subsection{Plasma measurements}

Plasma concentrations of alanine aminotransferase (ALT) [S.G.P.T ERBA kit, 120903; Baddi, India] and aspartate aminotransferase (AST) [S.G.O.T ERBA kit, B081717; Baddi, India] were measured with an enzymatic color test according to the manufacturer's protocols and were measured using an ERBA semi-automated biochemistry analyzer. Plasma insulin was measured using a rat enzyme-linked immunosorbent assay kit [ab100578, Abcam; Cambridge, MA, USA].

\subsection{Liver tumor necrosis factor-alpha levels}

The rat tumor necrosis factor-alpha (TNF- $\alpha$ ) ELISA assay was performed on liver homogenates according to the manufacturer's instructions [ab100785, Abcam; Cambridge, MA, USA] using a microplate reader.

\subsection{Statistical analyses}

Data were statistically analyzed by performing two-tailed unpaired $t$-tests using GraphPad Prism, version 6.0 for Windows. Data were expressed as mean \pm SEM and considered significant at $p<0.05$. $*, * *$ and $* * *$ indicate $p<0.05,0.01$ and 0.001 , respectively. 


\section{Results}

\subsection{Decreased inflammation after inhibition of extracellular CTSD in oxLDL-loaded primary mouse macrophages}

With the aim of exploring the influence of extracellular CTSD on inflammation in macrophages and to test the efficacy of the small-molecule inhibitor CTD-002, Wt BMDMs were isolated and incubated with oxLDL for $24 \mathrm{~h}$ to stimulate the secretion of CTSD. Subsequently, cells were treated with either carrier control (DMSO) or CTD-002 (100 $\mu \mathrm{M})$ for $4 \mathrm{~h}$, followed by $4 \mathrm{~h}$ stimulation with LPS. Tumor necrosis factor $\alpha$ (TNF $\alpha$ ) protein levels, a pro-inflammatory cytokine, measured from the medium of CTD-002-treated BMDMs, were significantly reduced compared to control cells (Figure 1A). Consistent with these results, gene expression levels of the pro-inflammatory cytokines Inf $\alpha$ and chemokine (C-X-C motif) ligand-2 ( $C \mathrm{Cl}$ ) significantly decreased in CTD-002-treated macrophages compared to control (DMSO)-treated cells (Figure 1B), confirming the reduction of inflammation upon treatment with CTD-002. Additionally, extracellular CTSD inhibition also improved cholesterol metabolism, as evidenced by the upregulation of cytochrome P450 27a1 (Cyp27a1), the main enzyme responsible for the conversion of cholesterol into bile acids (Figure $1 \mathrm{C}$ ). Similar data were also observed in control conditions without oxLDL and LPS stimulation, showing reduced $\operatorname{Tnf} \alpha$ and $\mathrm{Cc} / 2$ levels and improved lipid and energy metabolism as evidenced by Cyp27a1, acetyl-CoA acetyltransferase 2 (Acat2), carnitine palmitoyltransferase1 (Cpt1) and scavenger receptor A (Sr-a) expression after CTD-002 treatment (Figure S3). Collectively, these data indicate that modulating the activity of extracellular CTSD has beneficial effects on inflammation and cholesterol metabolism, at least in vitro.
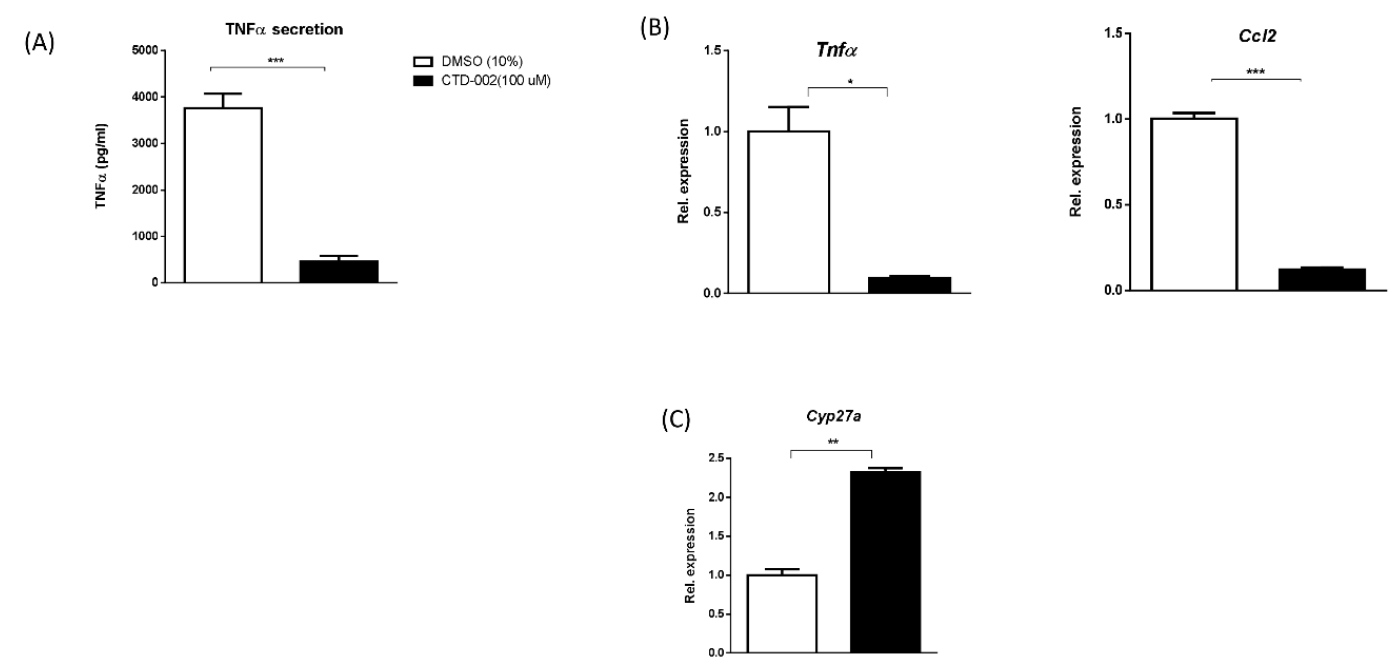

Figure 1 Effects of CTD-002 on inflammation and cholesterol metabolism in bone marrow-derived macrophages. (A) TNF $\alpha$ protein levels measured from supernatant of oxidized low-density lipoprotein (oxLDL)-loaded bone marrow-derived macrophages (BMDMs). (B) Gene expression of inflammation-related genes Tnf $\alpha$ and $C c / 2$ and (C) the cholesterol breakdown enzyme Cyp27a1 in oxLDL-loaded bone marrow-derived macrophages. Each bar represents a technical triplicate \pm SEM; * means $p<0.05, * * p<0.01$ and ${ }^{* * *} p<0.001$ compared to dimethyl sulfoxide (DMSO)treated BMDMs by means of two-tailed unpaired $t$-test. 


\subsection{Inhibition of macrophage derived extracellular CTSD reduces inflammation in HepG2 cells}

In order to confirm whether macrophage-derived extracellular CTSD influences neighboring parenchymal cells, hepatic HepG2 cells were incubated with a conditioned medium derived from macrophages that were treated either with carrier-control or with CTD-002 $(100 \mu \mathrm{M})$ for $4 \mathrm{~h}$. To boost the effects on inflammation, HepG2 cells received LPS for $4 \mathrm{~h}$. HepG2 cells that received the conditioned medium from macrophages that were treated with CTD-002 showed a trend towards reduced TNFa secretion (Figure 2A). Additionally, HepG2 gene expression levels of $C c / 2$ showed a decreasing trend compared to HepG2 cells that were incubated in the control-treated conditioned medium, while Tnf $\alpha$ did not show any effect (Figure 2B). Additionally, we also observed a reduced expression of the cluster of differentiation $36(C d 36)$ in hepatocytes receiving the conditioned medium that was derived from BMDMs treated with CTD-002, suggesting an effect on hepatocyte lipid metabolism (Figure S4). These in vitro findings suggest that macrophage derived extracellular CTSD affects the inflammatory status of neighboring parenchymal cells and that inhibiting extracellular CTSD activity reduces this inflammatory response.

(A)

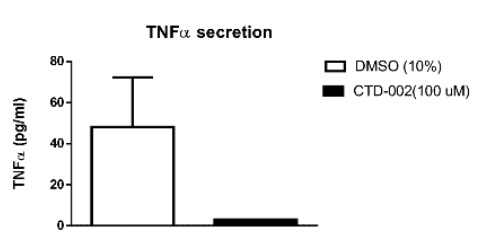

(B)
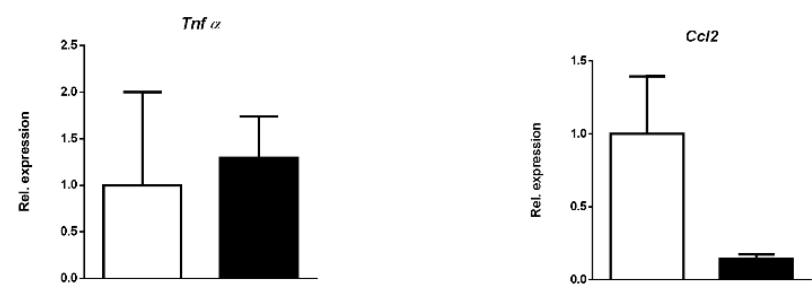

Figure 2 Effect of medium derived from CTD-002-treated BMDMs on HepG2 cells. (A) TNF $\alpha$ cytokine secretion of HepG2 cells cultured in a macrophage-conditioned medium that was treated with or without CTD-002. (B) Gene expression levels of Tnfa and CCl2 measured in HepG2 cells. Error bars represent mean \pm SEM.

\subsection{Improved metabolic features after inhibition of extracellular CTSD in HFD-fed Sprague-Dawley rats}

To elucidate the metabolic effects of extracellular CTSD inhibition in vivo, metabolic parameters were tested in HFD-fed SD rats that were injected with or without the CTD-002 inhibitor. Though food consumption was statistically similar among all experimental groups, a trend towards reduced food intake was apparent (Figure S5). By performing an H\&E staining, hepatic steatosis was assessed based on the scoring of fat vesicles in the liver (Figure S6). The impact of HFD CTD-002-treated HFD-fed SD rats showed a significant reduction in the amount of fat vesicles relative to control HFD-fed rats, demonstrating the therapeutic benefit of extracellular CTSD inhibition in the context of hepatic 
steatosis, a main feature of NAFLD (Figure 3A). The impact of the HFD on hepatic fat deposition was further confirmed by quantification of H\&E, Chromotrope Aniline Blue and Sirius Red staining, showing increased fat deposition with the HFD (Figure S7).

To further assess whether inhibition of extracellular CTSD influences pathological parameters related to NAFLD, plasma insulin levels were measured as an indicator of insulin sensitivity. Plasma insulin levels were significantly increased in HFD-fed SD rats compared to LFD-fed rats (Figure 3B). Further, CTD-002-treated HFD-fed rats had significantly lower plasma insulin levels compared to HFD-fed rats (Figure 3B). This finding suggests an improvement in insulin sensitivity, further emphasizing the metabolic benefit induced by inhibition of extracellular CTSD. Altogether, these data indicate that modulating extracellular CTSD activity improves metabolic features associated with NAFLD.

(A)

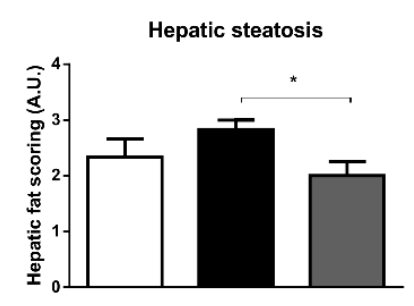

(B)

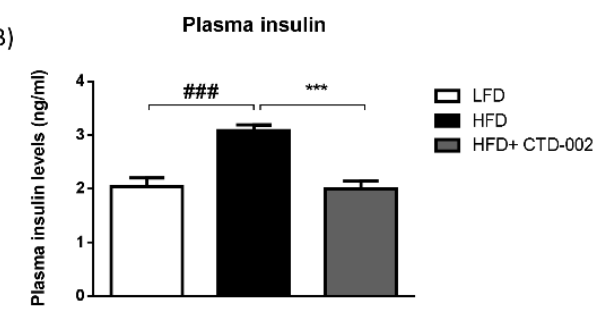

Figure 3 Metabolic parameters of high-fat diet (HFD)-fed Sprague-Dawley (SD) rats treated with or without the extracellular inhibitor CTD-002. (A) Scoring of hepatic steatosis by means of Hematoxylin-Eosin (H\&E) staining. (B) Plasma levels of insulin. Error bars represent mean \pm SEM; $n=6$ for each group; * represents $p<0.05$ and $* * * p<0.001$ compared to rats on HFD; \#\#\# represents $p<0.001$ compared to the rats on low-fat diet (LFD) by means of two-tailed unpaired $t$-test.

\subsection{Reduced liver damage after inhibition of extracellular CTSD in HFD-fed Sprague- Dawley rats}

To determine the effects of extracellular CTSD inhibition on hepatic inflammation, the protein levels of hepatic TNFa were measured in HFD-fed SD rats that were treated with or without the CTD-002 inhibitor of extracellular CTSD. CTD-002-treated HFD-fed SD rats showed a trend towards reduced hepatic TNF $\alpha$ levels $(p=0.06$ ) compared to the HFD-fed group (Figure 4A), thereby suggesting an improvement in hepatic inflammation. To further assess the effects of extracellular CTSD inhibition on liver damage, plasma aspartate aminotransferase (AST) and alanine aminotransferase (ALT) levels were measured. Upon treatment with CTD-002, HFD-fed SD rats showed a significant reduction in the aspartate transaminase/alanine transaminase (AST/ALT) ratio (Figure 4B), pointing towards an improvement in liver damage. 
(A)

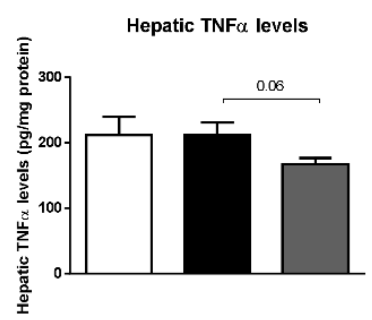

(B)

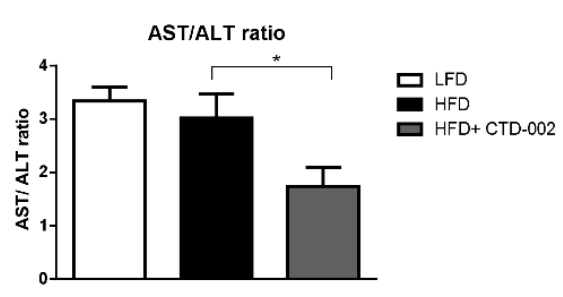

Figure 4 Hepatic TNF $\alpha$ levels and aspartate transaminase/alanine transaminase (AST/ALT) ratio in HFDfed SD rats treated with or without the extracellular inhibitor of cathepsin D (CTSD). (A) Hepatic TNF $\alpha$ levels. (B) Plasma transaminase levels. Error bars represent mean \pm SEM; $n=6$ for each group. * indicates $p<0.05$ compared to rats on the HFD.

\section{Discussion}

Treating NAFLD is of critical importance to prevent further progression to liver cirrhosis and endstage liver disease. The present study provides the first evidence that modulating extracellular CTSD activity can significantly improve metabolic parameters associated with NAFLD. These findings suggest the potential therapeutic benefits of targeting extracellular CTSD within the context of metabolic diseases and opens new perspectives for therapeutic interventions in NAFLD.

To elucidate the effects of extracellular CTSD inhibition in NAFLD, we have used SD rats that were fed a high-fat diet for three weeks. Sprague-Dawley rats are known to develop micro-vesicular steatosis with [21] or without inflammation [26], depending on the composition and duration of the HFD. Here, SD rats developed hepatic steatosis. Similar to the findings of Lieber and Ahmed et al. [21,26], no changes in relative liver weights were observed between different groups of rats (Table S2). Comparable to human NAFLD patients, we observed that rats on an HFD developed insulin resistance (IR) [27], as shown by elevated plasma insulin levels with the HFD. Indeed, it has been shown that insulin resistance is commonly associated with obesity-induced hepatic steatosis [28,29]. Furthermore, hepatic lipid accumulation is known to activate intrahepatic inflammatory pathways, which stimulate the pro-inflammatory cytokine production, in turn leading to both hepatic and peripheral insulin resistance [30,31]. Upon administration of the extracellular CTSD inhibitor, we observed that HFD-fed rats showed a significant reduction in plasma insulin levels and improvement of steatosis, two important characteristics of diet-induced NAFLD. Hence, these data provide initial evidence that extracellular CTSD activity is involved with the regulation of lipid metabolism and thereby controls insulin sensitivity. However, the trend towards reduced food intake suggests additional potential effects on energy expenditure, which should be further investigated in the future. Additionally, though hepatic damage and inflammation were not increased in SD rats fed an HFD, treatment with the extracellular CTSD inhibitor showed reduced hepatic TNF $\alpha$ levels and AST/ALT ratio, indicating an improvement in hepatic damage and inflammation. Moreover, we validated this finding in primary macrophages and in an in vitro setup in which hepatic cells were incubated with a macrophage-derived conditioned medium, an approach that has been previously used to investigate crosstalk between hepatic immune and parenchymal cells [32,33]. As such, our findings suggest that macrophage derived extracellular CTSD activity influences hepatic lipid metabolism and inflammation. However, despite the promising first results, the potential minor 
effects of intracellular CTSD and of the trend towards reduced food intake in the inhibitor-treated group suggest additional non-beneficial effects related to intracellular CTSD inhibition. To determine whether these non-beneficial metabolic effects are related directly to intracellular CTSD, a comparison with a compound with high permeability and low efflux (indicating that the compound mainly exerts its effects intracellularly) is necessary. Likewise, lower concentrations of the compound should be investigated in several NAFLD models before transition to the clinic is made.

Substantial evidence points towards the role of cathepsins in the context of NAFLD. Inactivation of cathepsin B in a dietary murine model of NAFLD prevented the development of hepatic steatosis [34]. Additionally, cathepsin B was found to be involved in hepatic injury and in progression of liver fibrosis $[35,36]$. Similarly, we have shown that lysosomal cholesterol accumulation inside Kupffer cells of Ldlr/- mice leads to increased CTSD activity in the liver [37]. CTSD is a key lysosomal protease that affects many fundamental functions in the cell. A reduction in cellular CTSD expression or catalytic activity leads to devastating neurodegenerative disorders [38,39]. In contrast, an increase in extracellular CTSD expression and activity is associated with many types of cancers [40-42]. Owing to this differential expression and secretion of CTSD in many pathophysiological processes, there is increased awareness for the extracellular fraction of CTSD to play a role in health and disease. However, to date, research primarily focuses on targeting the whole fraction of CTSD [43], which is known to disturb physiological processes, resulting in serious side effects [44]. Hence, in the present study, we specifically investigated the benefits of targeting the extracellular fraction of CTSD in the context of NAFLD.

Though the mechanisms underlying the secretion of CTSD are not yet known, changes in the lysosomal pH or lysosomal accumulation of poorly degradable lipids are known to cause mistargeting of CTSD into the extracellular milieu [45,46]. Additionally, cholesterol-filled lysosomes have been shown to induce disturbances in the lysosomal enzyme trafficking pathway that can potentially lead to increased levels of lysosomal enzymes in the plasma $[47,48]$. In vitro, particularly, the intracellular accumulation of the oxidized fraction of cholesterol has been shown to enhance extracellular secretion of CTSD [49,50]. Building further on this knowledge, we found in this study that targeting the activity of the extracellular fraction of CTSD in oxLDL-loaded macrophages improves inflammation and lipid metabolism, suggesting that macrophage-derived extracellular CTSD has a key role in lipid metabolism and inflammation.

Our observation that extracellular CTSD activity is involved with the pathogenesis of NAFLD implies that CTSD remains active in the circulation. Indeed, modulating the vacuolar type $\mathrm{H}+\mathrm{ATPase}$ pump by macrophages creates a more acidic pericellular space, in which lysosomal enzymes such as CTSD can remain active [51]. Moreover, several findings have implicated that upon secretion, anchorage of CTSD to the cell surface ameliorates its ability to interact with specific extracellular substrates [52]. The combination of an acidic pericellular environment and localization proximal to the cell proposes a potential mechanism by which extracellular CTSD activity can influence physiological processes. However, the question remains of which underlying mechanism is responsible for the observed improvements in lipid metabolism and inflammation upon inhibition of extracellular CTSD activity. In the current study, extracellular inhibition of CTSD upregulated the expression of Cyp27a1 in macrophages. Cyp27a1 is a gene encoding for an essential enzyme responsible for regulating cholesterol, fatty acid and bile acid metabolism through modulation of the mitochondrial P450 enzyme sterol 27-hydroxylase. In the liver, sterol 27-hydroxylase catalyzes the first step of the alternative bile acid biosynthetic pathway from cholesterol [53]. Previously, we have demonstrated 
the potential of Cyp27a1 to modulate intracellular cholesterol distribution in Kupffer cells [37]. Of note, Cyp27a1 has been shown to regulate ATP-binding cassette transporter A1 (ABCA1), an exporter of cellular lipids [54]. Based on this evidence, a possible explanation for our current findings is that the extracellular CTSD binds to one of the cell surface lipid transporters such as ABCA1 or scavenger receptors, which in turn blocks cholesterol transport, preventing regulation of cellular cholesterol and efflux pathways. It is therefore feasible that CTSD acts as an extracellular messenger interacting with an as yet unidentified cell surface receptor and regulates lipid homeostasis in addition to inflammation. In this regard, extracellular CTSD has recently been shown to be involved with ABCA1 regulation via influencing the low-density lipoprotein receptor-related protein 1 (LRP1) [55]. Additionally, we here observed differential expression of Cd36 and Sr-a upon extracellular CTSD inhibition, suggesting an involvement of these scavenger receptors. Moreover, modulating proteases is known to influence the transcriptional regulation of cholesterol handling via Srebp [56] and Nfe2/1 [57]. However, further studies are warranted to decipher the exact underlying mechanism of extracellular CTSD function.

In conclusion, we provide evidence for the first time that extracellular CTSD has a central role in the progression of NAFLD. In contrast to conventional therapeutic targeting of cathepsins, our data demonstrate that inhibiting specifically the extracellular fraction of CTSD can be a valuable therapeutic strategy for NAFLD. Further studies that investigate the downstream targets regulated by extracellular CTSD will provide deeper understanding of the mechanisms of NAFLD pathogenesis. 


\section{References}

1. Younossi, Z.M.; Koenig, A.B.; Abdelatif, D.; Fazel, Y.; Henry, L.; Wymer, M. Global epidemiology of nonalcoholic fatty liver disease-Meta-analytic assessment of prevalence, incidence, and outcomes. Hepatology 2016, 64, 73-84, doi:10.1002/hep.28431.

2. Tandra, S.; Yeh, M.M.; Brunt, E.M.; Vuppalanchi, R.; Cummings, O.W.; Unalp-Arida, A.; Wilson, L.A.; Chalasani, N. Presence and significance of microvesicular steatosis in nonalcoholic fatty liver disease. J Hepatol 2011, 55, 654-659, doi:10.1016/j.jhep.2010.11.021.

3. Takahashi, Y.; Fukusato, T. Histopathology of nonalcoholic fatty liver disease/nonalcoholic steatohepatitis. World J Gastroenterol 2014, 20, 15539-15548, doi:10.3748/wjg.v20.i42.15539.

4. Contos, M.J.; Choudhury, J.; Mills, A.S.; Sanyal, A.J. The histologic spectrum of nonalcoholic fatty liver disease. Clin Liver Dis 2004, 8, 481-500, vii, doi:10.1016/j.cld.2004.04.013.

5. Musso, G.; Cassader, M.; Rosina, F.; Gambino, R. Impact of current treatments on liver disease, glucose metabolism and cardiovascular risk in non-alcoholic fatty liver disease (NAFLD): A systematic review and meta-analysis of randomised trials. Diabetologia 2012, 55, 885-904, doi:10.1007/s00125-011-2446-4.

6. Cheung, O.; Sanyal, A.J. Abnormalities of lipid metabolism in nonalcoholic fatty liver disease. Semin Liver Dis 2008, 28, 351-359, doi:10.1055/s-0028-1091979.

7. Lindstedt, L.; Lee, M.; Oorni, K.; Bromme, D.; Kovanen, P.T. Cathepsins F and S block HDL3-induced cholesterol efflux from macrophage foam cells. Biochemical and Biophysical Research Communications 2003, 312, 1019-1024, doi:10.1016/j.bbrc.2003.11.020.

8. Tertov, V.V.; Orekhov, A.N. Metabolism of native and naturally occurring multiple modified low-density lipoprotein in smooth muscle cells of human aortic intima. Experimental and Molecular Pathology 1997, 64, 127-145, doi:DOI 10.1006/exmp.1997.2216.

9. Thelen, A.M.; Zoncu, R. Emerging Roles for the Lysosome in Lipid Metabolism. Trends Cell Biol 2017, 27, 833-850, doi:10.1016/j.tcb.2017.07.006.

10. Lutgens, S.P.M.; Cleutjens, K.B.J.M.; Daemen, M.J.A.P.; Heeneman, S. Cathepsin cysteine proteases in cardiovascular disease. Faseb Journal 2007, 21, 3029-3041, doi:10.1096/fj.06-7924com.

11. Benes, P.; Vetvicka, V.; Fusek, M. Cathepsin D--many functions of one aspartic protease. Crit Rev Oncol Hematol 2008, 68, 12-28, doi:10.1016/j.critrevonc.2008.02.008.

12. Moallem, S.A.; Nazemian, F.; Eliasi, S.; Alamdaran, S.A.; Shamsara, J.; Mohammadpour, A.H. Correlation between cathepsin $D$ serum concentration and carotid intima-media thickness in hemodialysis patients. International Urology and Nephrology 2011, 43, 841-848, doi:10.1007/s11255-010-9729-4.

13. Snir, J.A.; Suchy, M.; St Lawrence, K.; Hudson, R.H.E.; Pasternak, S.H.; Bartha, R. Prolonged In Vivo Retention of a Cathepsin D Targeted Optical Contrast Agent in a Mouse Model of Alzheimer's Disease. Journal of Alzheimers Disease 2015, 48, 73-87, doi:10.3233/Jad-150123.

14. Briozzo, P.; Badet, J.; Capony, F.; Pieri, I.; Montcourrier, P.; Barritault, D.; Rochefort, H. MCF7 mammary cancer cells respond to bFGF and internalize it following its release from extracellular matrix: A permissive role of cathepsin D. Exp Cell Res 1991, 194, 252-259.

15. Porter, K.; Lin, Y.Z.; Liton, P.B. Cathepsin B Is Up-Regulated and Mediates Extracellular Matrix Degradation in Trabecular Meshwork Cells Following Phagocytic Challenge. PLOS ONE 2013, 8, doi:ARTN e6866810.1371/journal.pone.0068668.

16. Naseem, R.H.; Hedegard, W.; Henry, T.D.; Lessard, J.; Sutter, K.; Katz, S.A. Plasma cathepsin D isoforms and their active metabolites increase after myocardial infarction and contribute to plasma renin activity. Basic Research in Cardiology 2005, 100, 139-146, doi:10.1007/s00395-004-0499-3.

17. Walenbergh, S.M.; Houben, T.; Hendrikx, T.; Jeurissen, M.L.; van Gorp, P.J.; Vreugdenhil, A.C.; Adriaanse, M.P.; Buurman, W.A.; Hofker, M.H.; Mosca, A., et al. Plasma cathepsin D levels: A novel tool to predict pediatric hepatic inflammation. Am J Gastroenterol 2015, 110, 462-470, doi:10.1038/ajg.2015.29.

18. Walenbergh, S.M.; Houben, T.; Rensen, S.S.; Bieghs, V.; Hendrikx, T.; van Gorp, P.J.; Oligschlaeger, Y.; Jeurissen, M.L.; Gijbels, M.J.; Buurman, W.A., et al. Plasma cathepsin D correlates with histological classifications of fatty liver disease in adults and responds to intervention. Sci Rep 2016, 6, 38278, doi:10.1038/srep38278. 
19. Houben, T.; Oligschlaeger, Y.; Hendrikx, T.; Bitorina, A.V.; Walenbergh, S.M.A.; van Gorp, P.J.; Gijbels, M.J.J.; Friedrichs, S.; Plat, J.; Schaap, F.G., et al. Cathepsin D regulates lipid metabolism in murine steatohepatitis. Sci Rep 2017, 7, 3494, doi:10.1038/s41598-017-03796-5.

20. Marciniszyn, J., Jr.; Hartsuck, J.A.; Tang, J. Mode of inhibition of acid proteases by pepstatin. J Biol Chem 1976, 251, 7088-7094.

21. Lieber, C.S.; Leo, M.A.; Mak, K.M.; Xu, Y.; Cao, Q.; Ren, C.; Ponomarenko, A.; DeCarli, L.M. Model of nonalcoholic steatohepatitis. Am J Clin Nutr 2004, 79, 502-509, doi:10.1093/ajcn/79.3.502.

22. Dixon, S.L.; Smondyrev, A.M.; Knoll, E.H.; Rao, S.N.; Shaw, D.E.; Friesner, R.A. PHASE: A new engine for pharmacophore perception, 3D QSAR model development, and 3D database screening: 1. Methodology and preliminary results. Journal of Computer-Aided Molecular Design 2006, 20, 647-671, doi:10.1007/s10822-006-9087-6.

23. Dixon, S.L.; Smondyrev, A.M.; Rao, S.N. PHASE: A novel approach to pharmacophore modeling and 3D database searching. Chemical Biology \& Drug Design 2006, 67, 370-372, doi:10.1111/j.1747-0285.2006.00384.x.

24. Friesner, R.A.; Banks, J.L.; Murphy, R.B.; Halgren, T.A.; Klicic, J.J.; Mainz, D.T.; Repasky, M.P.; Knoll, E.H.; Shelley, M.; Perry, J.K., et al. Glide: A new approach for rapid, accurate docking and scoring. 1. Method and assessment of docking accuracy. J Med Chem 2004, 47, 1739-1749, doi:10.1021/jm0306430.

25. Halgren, T.A.; Murphy, R.B.; Friesner, R.A.; Beard, H.S.; Frye, L.L.; Pollard, W.T.; Banks, J.L. Glide: A new approach for rapid, accurate docking and scoring. 2. Enrichment factors in database screening. J Med Chem 2004, 47, 1750-1759, doi:10.1021/jm030644s.

26. Ahmed, U.; Redgrave, T.G.; Oates, P.S. Effect of dietary fat to produce non-alcoholic fatty liver in the rat. J Gastroenterol Hepatol 2009, 24, 1463-1471, doi:10.1111/j.1440-1746.2009.05870.x.

27. Zou, Y.; Li, J.; Lu, C.; Wang, J.; Ge, J.; Huang, Y.; Zhang, L.; Wang, Y. High-fat emulsion-induced rat model of nonalcoholic steatohepatitis. Life Sci 2006, 79, 1100-1107, doi:10.1016/j.Ifs.2006.03.021.

28. Kitade, H.; Chen, G.; Ni, Y.; Ota, T. Nonalcoholic Fatty Liver Disease and Insulin Resistance: New Insights and Potential New Treatments. Nutrients 2017, 9, doi:10.3390/nu9040387.

29. Perry, R.J.; Samuel, V.T.; Petersen, K.F.; Shulman, G.I. The role of hepatic lipids in hepatic insulin resistance and type 2 diabetes. Nature 2014, 510, 84-91, doi:10.1038/nature13478.

30. Samuel, V.T.; Liu, Z.X.; Qu, X.Q.; Elder, B.D.; Bilz, S.; Befroy, D.; Romanelli, A.J.; Shulman, G.I. Mechanism of hepatic insulin resistance in non-alcoholic fatty liver disease. Journal of Biological Chemistry 2004, 279, 32345-32353, doi:10.1074/jbc.M313478200.

31. Yamaguchi, K.; Nishimura, T.; Ishiba, H.; Seko, Y.; Okajima, A.; Fujii, H.; Tochiki, N.; Umemura, A.; Moriguchi, M.; Sumida, Y., et al. Blockade of interleukin 6 signalling ameliorates systemic insulin resistance through upregulation of glucose uptake in skeletal muscle and improves hepatic steatosis in high-fat diet fed mice. Liver International 2015 , 35, 550-561, doi:10.1111/liv.12645.

32. Pardo, V.; Gonzalez-Rodriguez, A.; Guijas, C.; Balsinde, J.; Valverde, A.M. Opposite cross-talk by oleate and palmitate on insulin signaling in hepatocytes through macrophage activation. J Biol Chem 2015, 290, 11663-11677, doi:10.1074/jbc.M115.649483.

33. Melino, M.; Gadd, V.L.; Walker, G.V.; Skoien, R.; Barrie, H.D.; Jothimani, D.; Horsfall, L.; Jones, A.; Sweet, M.J.; Thomas, G.P., et al. Macrophage secretory products induce an inflammatory phenotype in hepatocytes. World J Gastroenterol 2012, 18, 1732-1744, doi:10.3748/wjg.v18.i15.1732.

34. Feldstein, A.E.; Werneburg, N.W.; Canbay, A.; Guicciardi, M.E.; Bronk, S.F.; Rydzewski, R.; Burgart, L.J.; Gores, G.J. Free fatty acids promote hepatic lipotoxicity by stimulating TNF-alpha expression via a lysosomal pathway. Hepatology 2004, 40, 185-194, doi:10.1002/hep.20283.

35. Li, Z.Z.; Berk, M.; McIntyre, T.M.; Gores, G.J.; Feldstein, A.E. The lysosomal-mitochondrial axis in free fatty acidinduced hepatic lipotoxicity. Hepatology 2008, 47, 1495-1503, doi:10.1002/hep.22183.

36. Moles, A.; Tarrats, N.; Fernandez-Checa, J.C.; Mari, M. Cathepsins B and D Drive Hepatic Stellate Cell Proliferation and Promote Their Fibrogenic Potential. Hepatology 2009, 49, 1297-1307, doi:10.1002/hep.22753.

37. Bieghs, V.; Hendrikx, T.; van Gorp, P.J.; Verheyen, F.; Guichot, Y.D.; Walenbergh, S.M.A.; Jeurissen, M.L.J.; Gijbels, M.; Rensen, S.S.; Bast, A., et al. The Cholesterol Derivative 27-Hydroxycholesterol Reduces Steatohepatitis in Mice. Gastroenterology 2013, 144, 167-U291, doi:10.1053/j.gastro.2012.09.062.

38. Saftig, P.; Hetman, M.; Schmahl, W.; Weber, K.; Heine, L.; Mossmann, H.; Koster, A.; Hess, B.; Evers, M.; von Figura, K., et al. Mice deficient for the lysosomal proteinase cathepsin $D$ exhibit progressive atrophy of the intestinal mucosa and profound destruction of lymphoid cells. EMBO J 1995, 14, 3599-3608. 


\section{Chapter 3}

39. Koike, M.; Nakanishi, H.; Saftig, P.; Ezaki, J.; Isahara, K.; Ohsawa, Y.; Schulz-Schaeffer, W.; Watanabe, T.; Waguri, S.; Kametaka, S., et al. Cathepsin D deficiency induces lysosomal storage with ceroid lipofuscin in mouse CNS neurons. J Neurosci 2000, 20, 6898-6906.

40. Glondu, M.; Coopman, P.; Laurent-Matha, V.; Garcia, M.; Rochefort, H.; Liaudet-Coopman, E. A mutated cathepsin-D devoid of its catalytic activity stimulates the growth of cancer cells. Oncogene 2001, 20, 6920-6929, doi:10.1038/sj.onc.1204843.

41. Vetvicka, V.; Vetvickova, J.; Fusek, M. Effect of procathepsin D and its activation peptide on prostate cancer cells. Cancer Lett 1998, 129, 55-59.

42. Vetvicka, V.; Vetvickova, J.; Benes, P. Role of enzymatically inactive procathepsin D in lung cancer. Anticancer Res 2004, 24, 2739-2743.

43. Dubey, V.; Luqman, S. Cathepsin D as a Promising Target for the Discovery of Novel Anticancer Agents. Current Cancer Drug Targets 2017, 17, 404-422, doi:10.2174/1568009616666161229145115.

44. Ruan, H.; Hao, S.; Young, P.; Zhang, H. Targeting Cathepsin B for Cancer Therapies. Horiz Cancer Res 2015, 56, $23-40$.

45. Settembre, C.; Di Malta, C.; Polito, V.A.; Garcia Arencibia, M.; Vetrini, F.; Erdin, S.; Erdin, S.U.; Huynh, T.; Medina, D.; Colella, P., et al. TFEB links autophagy to lysosomal biogenesis. Science 2011, 332, 1429-1433, doi:10.1126/science.1204592.

46. Rosenfeld, M.G.; Kreibich, G.; Popov, D.; Kato, K.; Sabatini, D.D. Biosynthesis of lysosomal hydrolases: Their synthesis in bound polysomes and the role of co- and post-translational processing in determining their subcellular distribution. J Cell Biol 1982, 93, 135-143.

47. Ungewickell, A.J.; Majerus, P.W. Increased levels of plasma lysosomal enzymes in patients with Lowe syndrome. Proc Natl Acad Sci U S A 1999, 96, 13342-13344.

48. Hultberg, B.; Isaksson, A.; Sjoblad, S.; Ockerman, P.A. Acid hydrolases in serum from patients with lysosomal disorders. Clin Chim Acta 1980, 100, 33-38.

49. Hoppe, G.; O'Neil, J.; Hoff, H.F.; Sears, J. Products of lipid peroxidation induce missorting of the principal lysosomal protease in retinal pigment epithelium. Biochimica Et Biophysica Acta-Molecular Basis of Disease 2004, 1689, 33-41, doi:10.1016/j.bbadis.2004.01.004.

50. Li, W.; Yuan, X.M.; Olsson, A.G.; Brunk, U.T. Uptake of oxidized LDL by macrophages results in partial lysosomal enzyme inactivation and relocation. Arteriosclerosis Thrombosis and Vascular Biology 1998, 18, 177-184, doi:Doi 10.1161/01.Atv.18.2.177.

51. Reddy, V.Y.; Zhang, Q.Y.; Weiss, S.J. Pericellular mobilization of the tissue-destructive cysteine proteinases, cathepsins B, L, and S, by human monocyte-derived macrophages. Proc Natl Acad Sci U S A 1995, 92, 3849-3853.

52. Neurath, H. Evolution of proteolytic enzymes. Science 1984, 224, 350-357.

53. Dubrac, S.; Lear, S.R.; Ananthanarayanan, M.; Balasubramaniyan, N.; Bollineni, J.; Shefer, S.; Hyogo, H.; Cohen, D.E.; Blanche, P.J.; Krauss, R.M., et al. Role of CYP27A in cholesterol and bile acid metabolism. J Lipid Res 2005, 46, 76-85, doi:10.1194/jlr.M400219-JLR200.

54. Oram, J.F. HDL apolipoproteins and ABCA1: Partners in the removal of excess cellular cholesterol. Arterioscler Thromb Vasc Biol 2003, 23, 720-727, doi:10.1161/01.ATV.0000054662.44688.9A.

55. Oldoni, F.; van Capelleveen, J.C.; Dalila, N.; Wolters, J.C.; Heeren, J.; Sinke, R.J.; Hui, D.Y.; Dallinga-Thie, G.M.; FrikkeSchmidt, R.; Hovingh, K.G., et al. Naturally Occurring Variants in LRP1 (Low-Density Lipoprotein Receptor-Related Protein 1) Affect HDL (High-Density Lipoprotein) Metabolism Through ABCA1 (ATP-Binding Cassette A1) and SR-B1 (Scavenger Receptor Class B Type 1) in Humans. Arterioscler Thromb Vasc Biol 2018, 38, 1440-1453, doi:10.1161/ATVBAHA.117.310309.

56. Brown, M.S.; Goldstein, J.L. The SREBP pathway: Regulation of cholesterol metabolism by proteolysis of a membranebound transcription factor. Cell 1997, 89, 331-340.

57. Widenmaier, S.B.; Snyder, N.A.; Nguyen, T.B.; Arduini, A.; Lee, G.Y.; Arruda, A.P.; Saksi, J.; Bartelt, A.; Hotamisligil, G.S. NRF1 Is an ER Membrane Sensor that Is Central to Cholesterol Homeostasis. Cell 2017, 171, 1094-+, doi:10.1016/j.cell.2017.10.003. 


\section{Supplemental material}

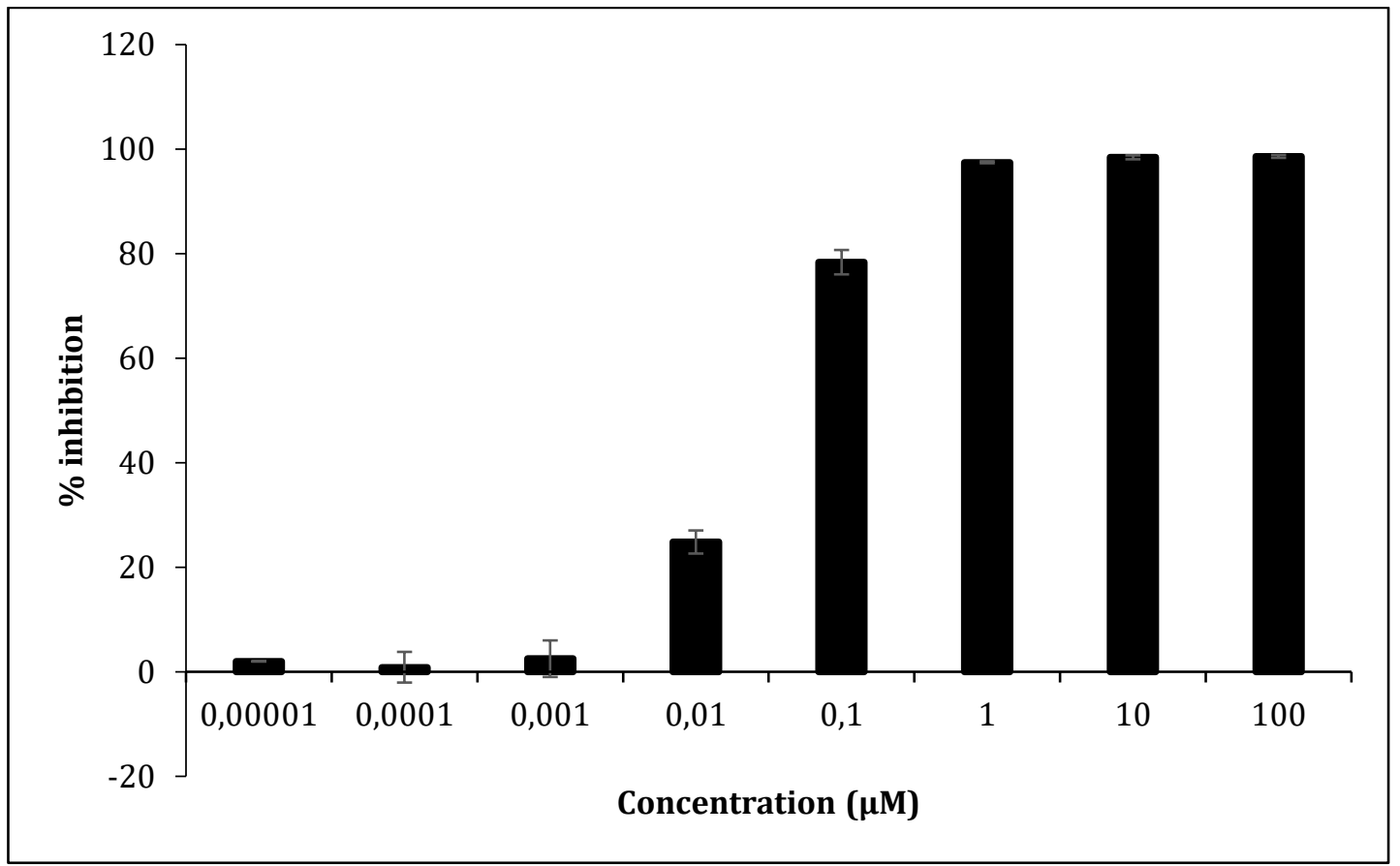

Figure S1 Inhibitory activity of CTD-002 on CTSD activity

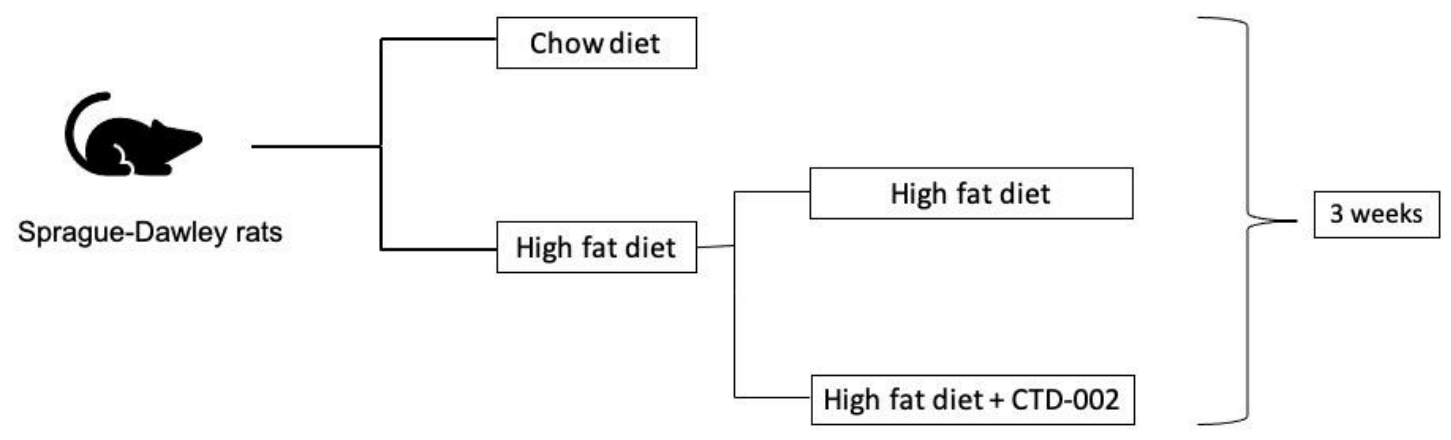

Figure S2 Schematic representation of the in-vivo setup 


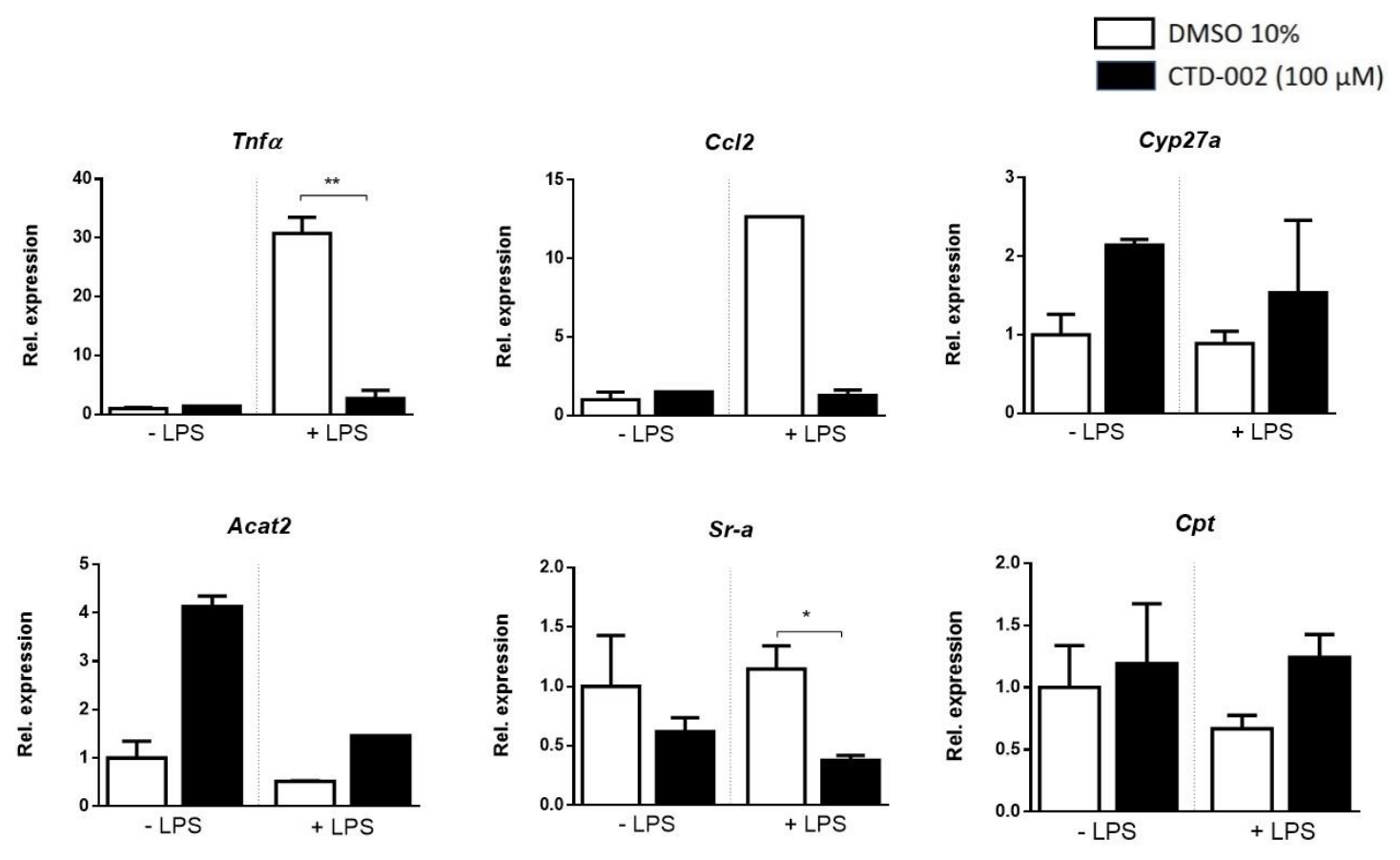

Figure S3 Effect of CTD-002 in bone marrow-derived macrophages under control conditions

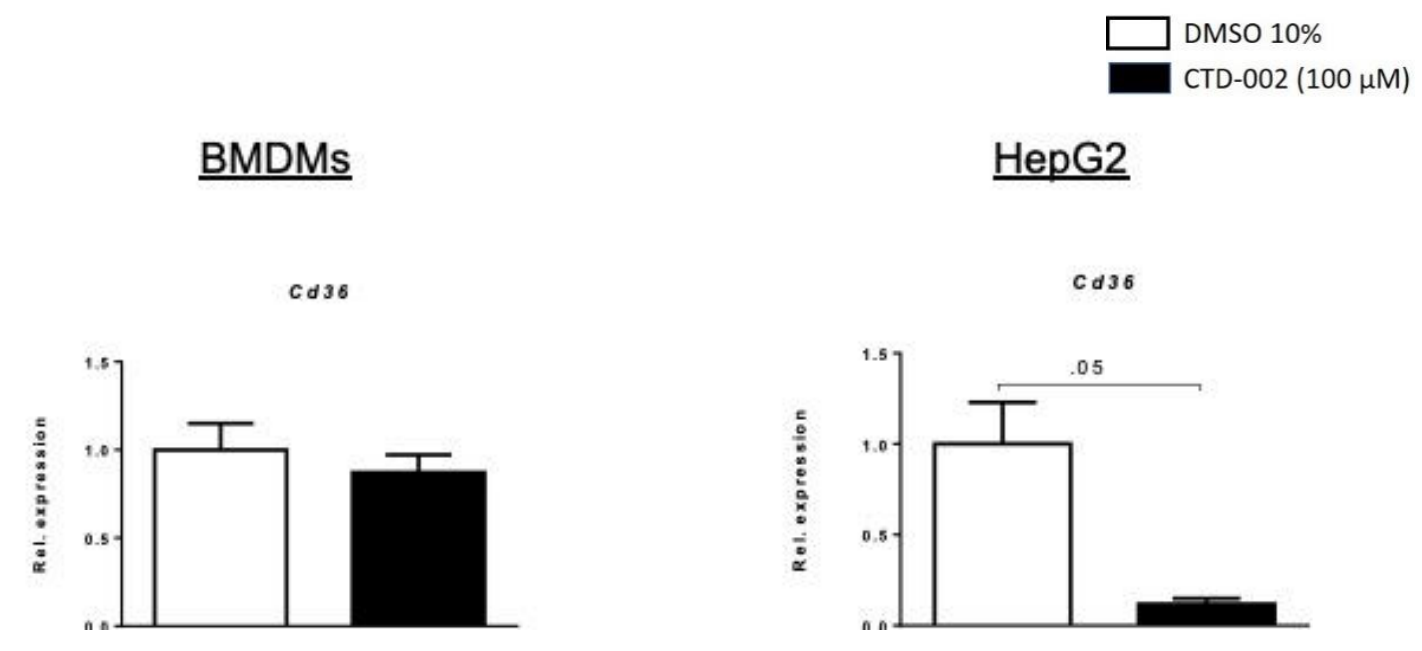

Figure S4 Cd36 gene expression levels of BMDMs and HepG2 
Food consumption

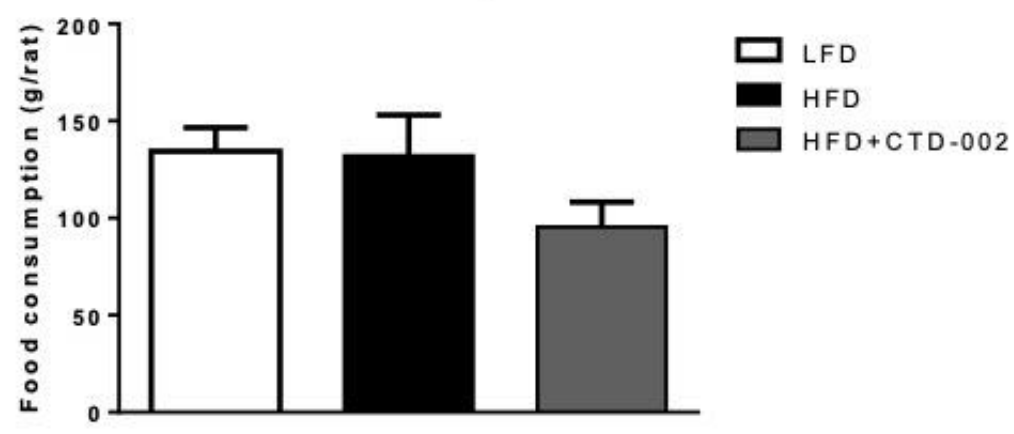

Figure S5 Food consumption of Sprague-Dawley rats

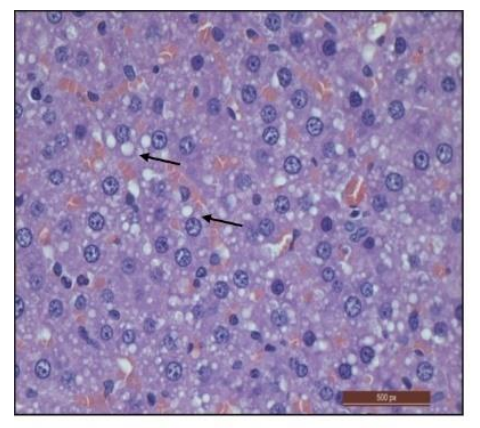

Low fat diet

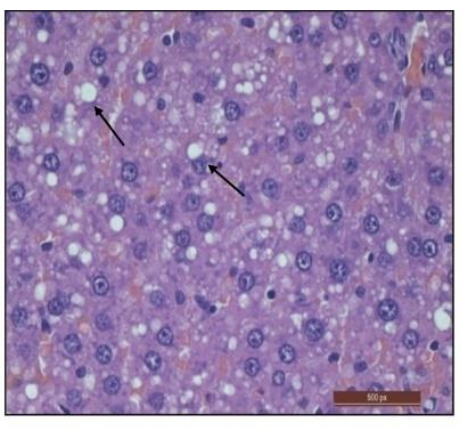

High fat diet

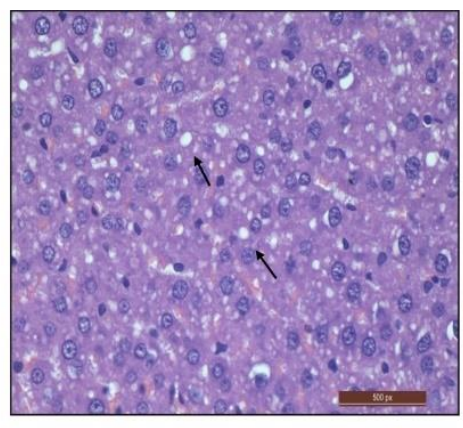

High fat diet + CTD-002

Figure S6 Representative images of fat droplets stained by haematoxylin and eosin staining
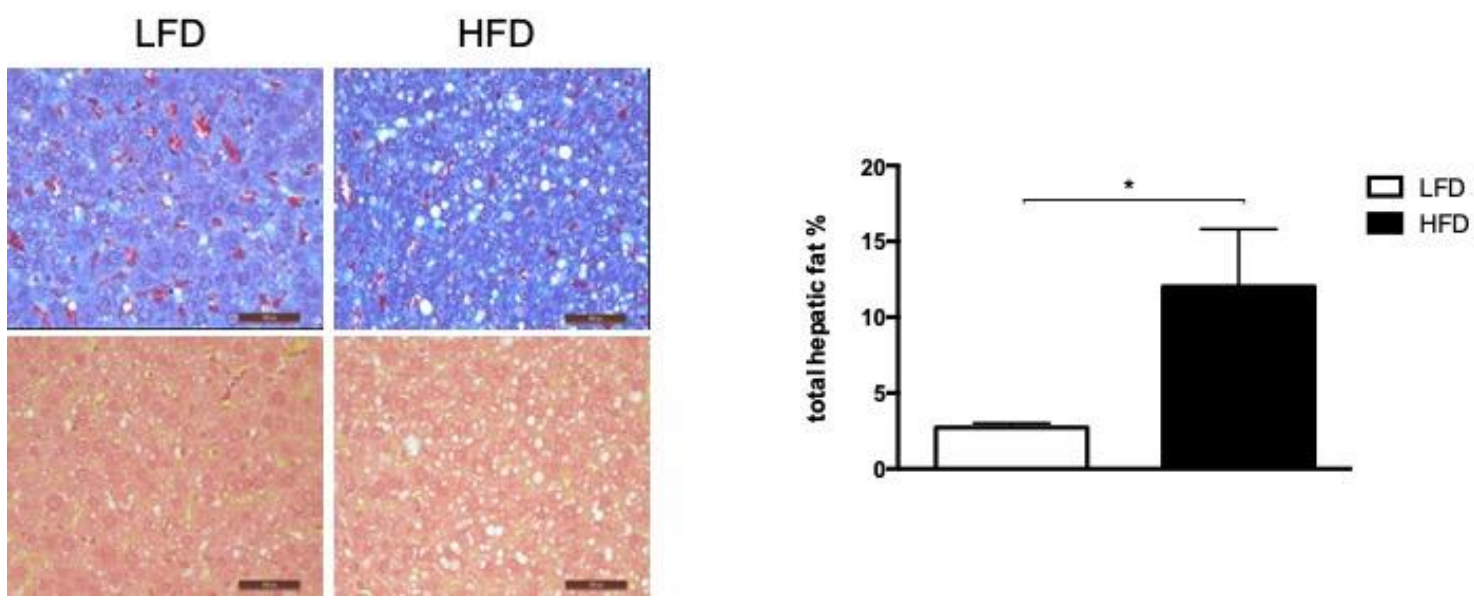

Figure S7 Impact of high-fat diet on hepatic fat deposition in Sprague-Dawley rats 
Table S1: Composition of low and high fat diets

\begin{tabular}{|c|c|c|}
\hline Ingredients & Low fat diet (gm) & High fat diet (gm) \\
\hline Casein & 200 & 200 \\
\hline L-Cystine & 3 & 3 \\
\hline Corn starch & 506 & 0 \\
\hline Maltodextrin 10 & 125 & 125 \\
\hline Sucrose & 68.8 & 68.8 \\
\hline Cellulose & 50 & 50 \\
\hline Soybean Oil & 25 & 25 \\
\hline Lard* & 20 & 245 \\
\hline DiCalcium Phosphate & 13 & 13 \\
\hline Calcium Carbonate & 5.5 & 5.5 \\
\hline Choline Bitartrate & 2 & 2 \\
\hline Potassium Citrate & 16.5 & 16.5 \\
\hline Mineral Mix & 10 & 10 \\
\hline Vitamin Mix & 10 & 10 \\
\hline FD\&C Blue Dye & 0.01 & 0.05 \\
\hline FD\&C yellow Dye & 0.04 & 0 \\
\hline \multicolumn{3}{|c|}{ *Typical analysis of cholesterol in lard $=0.72 \mathrm{mg} /$ gram } \\
\hline
\end{tabular}

Table S2: Relative liver weight (per $100 \mathrm{~g}$ of body weight) of the experimental groups of rats

\begin{tabular}{cc}
\hline Group & Liver weight $\mathbf{m g} / \mathbf{1 0 0 g}$ body weight (Mean \pm SD) \\
\hline LFD & $3.67 \pm 0.61$ \\
\hline HFD & $3.73 \pm 0.27$ \\
\hline HFD + CTD-002 & $3.73 \pm 0.47$ \\
\hline
\end{tabular}




\section{Chapter 4}

Inhibition of extracellular cathepsin D reduces hepatic lipid accumulation and leads to mild changes in inflammation in NASH mice

Tulasi Yadati, Tom Houben, Albert Bitorina, Yvonne Oligschlaeger, Marion J. Gijbels, Ronny Mohren, Dieter Lütjohann, Princy Khurana, Sandeep Goyal, Aditya Kulkarni, Jan Theys, Berta

Cillero-Pastor, Ronit Shiri-Sverdlov 


\section{Chapter 4}

\section{Abstract \\ Background \& Aims}

The lysosomal enzyme, cathepsin D (CTSD) has been implicated in the pathogenesis of non-alcoholic steatohepatitis (NASH), a disease characterized by hepatic steatosis and inflammation. We have previously demonstrated that specific inhibition of the extracellular CTSD leads to improved metabolic features in Sprague- Dawley rats with steatosis. However, the individual roles of extracellular and intracellular CTSD in NASH are not yet known. In the current study, we evaluated the underlying mechanisms of extracellular and intracellular CTSD fractions in NASH-related metabolic inflammation using specific small-molecule inhibitors.

\section{Methods}

Low-density lipoprotein receptor knock-out (Ldlr-/-) mice were fed a high-fat, high cholesterol (HFC) diet for ten weeks to induce NASH. Further, to investigate the effects of CTSD inhibition, mice were injected either with an intracellular (GA-12) or extracellular (CTD-002) CTSD inhibitor or vehicle control at doses of $50 \mathrm{mg} / \mathrm{kg}$ body weight subcutaneously once in two days for ten weeks.

\section{Results}

Ldlr-/- mice treated with extracellular CTSD inhibitor showed reduced hepatic lipid accumulation and an associated increase in faecal bile acid levels as compared to intracellular CTSD inhibitor-treated mice. Furthermore, in contrast to intracellular CTSD inhibition, extracellular CTSD inhibition switched the systemic immune status of the mice to an anti-inflammatory profile. In line, label-free mass spectrometry-based proteomics revealed that extra- and intracellular CTSD fractions modulate proteins belonging to distinct metabolic pathways.

\section{Conclusion}

We have provided clinically translatable evidence that extracellular CTSD inhibition shows some beneficial metabolic and systemic inflammatory effects which are distinct from intracellular CTSD inhibition. Considering that intracellular CTSD inhibition is involved in essential physiological processes, specific inhibitors capable of blocking extracellular CTSD activity, can be promising and safe NASH drugs. 


\section{Introduction}

Non-alcoholic fatty liver disease [NAFLD] is an expanding global health burden. NAFLD is an umbrella term that encompasses liver conditions ranging from steatosis to non-alcoholic steatohepatitis [NASH] and cirrhosis. While steatosis represents abnormal fat accumulation in the liver, NASH is steatosis accompanied by inflammation with or without fibrotic scarring [1]. NASH can further progress to end-stage liver diseases such as cirrhosis and hepatocellular carcinoma [2]. Though there have been considerable advances in recent years in elucidating the pathophysiological mechanisms involved in $\mathrm{NASH}$, much of the underlying mechanisms remain elusive, which is hampering drug development [3, 4]. Currently, no Food and Drug Administration (FDA)-approved drugs are available for this disease $[5,6]$ and therefore, there is an urgent need for effective treatment options to manage the complex pathogenesis of NASH.

We have previously demonstrated the relationship between lysosomal lipid accumulation in macrophages and hepatic inflammation during NASH $[7,8]$. Excessive accumulation of lipids in the lysosomes or changes in lysosomal $\mathrm{pH}$ leads to lysosomal dysfunction which subsequently disrupts the trafficking and sorting of lysosomal proteases causing their leakage into the circulation [9-12]. In particular, the secreted lysosomal proteases, cathepsins, are known to participate in several inflammatory responses. For example, in bone disorders and cancer, cathepsins secretion is known to be a common part of the inflammatory response $[13,14]$.

CTSD is an aspartyl lysosomal protease synthesized in the rough endoplasmic reticulum from where it is sorted to lysosomes [15]. As a result of excess lipids, extracellular CTSD secretion is elevated, where its increased activity in the plasma is known to be associated with metabolic inflammatory disorders such as NAFLD [16], atherosclerosis [17] and type 2 diabetes [18]. We have established that inhibition of CTSD proteolytic activity using the generic aspartyl protease inhibitor pepstatin A ameliorated NASH [19]. However, given the essential role of intracellular CTSD fraction in important cellular processes such as protein degradation and autophagy, its inhibition can have harmful effects if applied in humans $[20,21]$. Taking this into consideration, we have recently designed and tested a novel non- toxic inhibitor, CTD-002, that specifically targets the extracellular CTSD fraction. Our promising results showed that CTD-002 reduces hepatic steatosis and improves insulin sensitivity in Sprague-Dawley rats [22]. While we successfully demonstrated the importance of extracellular CTSD activity in steatosis, the underlying mechanism of extracellular CTSD action compared to intracellular CTSD inhibition in NASH- associated hepatic inflammation was never investigated. Such evaluation is important in order to establish extracellular CTSD as an effective and less-toxic treatment for NASH.

By using specific small-molecule inhibitors of CTSD, we demonstrated that while intracellular CTSD is involved in essential processes such as mitochondrial oxidative phosphorylation and electron transport function, extracellular CTSD is mainly involved in pathways related to lipids and inflammation. In line, extracellular CTSD inhibition led to increased beneficial effects in NASH compared to intracellular CTSD inhibition, including reduced hepatic triglyceride levels, increased faecal bile acids and reduced systemic inflammation. 


\section{Materials and methods}

\subsection{Primary culture of bone marrow-derived macrophages (BMDMs)}

BMDMs were isolated from the tibiae and femurs of wildtype C57BL/6 mice. Cells were cultured in RPMI-1640 (GIBCO Invitrogen, Breda, the Netherlands) with 10\% heat-inactivated fetal calf serum (Bodinco B.V. Alkmaar, The Netherlands), penicillin $(100 \mathrm{U} / \mathrm{ml})$, streptomycin $(100 \mathrm{mg} / \mathrm{ml})$ and Lglutamine (2 mM) (all GIBCO Invitrogen, Breda, The Netherlands), supplemented with 20\% L929conditioned medium (LCM) for 8-9 days to generate BMDMs. After attachment, macrophages were seeded at 350,000 cells per well in 24-well plates and incubated for 24 hours with oxidized lowdensity lipoprotein (oxLDL) $(25 \mathrm{mg} / \mathrm{ml}$; Alfa Aesar, Wardhill, MA, USA). Subsequently, macrophages were treated with $100 \mu$ M CTD-002 or GA-12 (Aten Porus Lifesciences Pvt Ltd., India) or with vehicle control [0.1\% dimethyl sulphoxide (DMSO)] for 4 hours. CTD-002 is a potent small-molecule active site inhibitor of CTSD designed based on the binding interactions of pepstatin A. Docking studies showed that CTD-002 interacts with polar side chain residues of ASP33 and GLY133 of ligand binding site of the human CTSD (PDB ID: 4od9).

\subsection{Mice, diet and intervention}

Ldlr-/- mice, on a C57BL/ 6 background, were housed under standard conditions and given free access to food and water. Experiments were performed according to the Dutch regulation and approved by the Committee for Animal Welfare of Maastricht University (Project license number: AVD107002016743; working protocol: 2016-003-003). Female Ldlr-/- mice (8-14 weeks of age) were fed high-fat, high-cholesterol (HFC) diet (containing 21\% milk butter, $0.2 \%$ cholesterol, $46 \%$ carbohydrates and $17 \%$ casein; SAFE, Augy, France) for ten weeks and were subsequently divided into three groups ( $n=17-20$ per group; schematic overview in Figure S1). To evaluate the potential side effects of intracellular CTSD inhibition, a specific small-compound inhibitor, GA-12, was designed and used in this study. HFC-fed mice were subcutaneously injected with extracellular CTSD inhibitor (CTD- 002; $50 \mathrm{mg} / \mathrm{kg}$ body weight) or intracellular CTSD inhibitor, GA-12 (50 mg/kg body weight) once every two days. Vehicle treatment (5\% DMSO, 40\% PEG400, 10\% Ethanol, 45\% Saline) served as control. Ldlr-/- mice fed a regular chow diet (9\% fat, $67 \%$ carbohydrates and $24 \%$ protein) for 10 weeks were included as a control group for NASH disease phenotype $(n=20)$. At the end of treatment, animals were euthanized by $\mathrm{CO} 2$ inhalation. Collection of blood and tissue specimens, RNA isolation and cDNA synthesis were performed as described previously $[7,19]$.

\subsection{Liver lipid analysis}

Liver tissue was isolated and snap-frozen in liquid nitrogen and stored at $-80^{\circ} \mathrm{C}$. The biochemical determination of liver cholesterol and triglyceride levels were carried out as described previously [7]. Briefly, $50 \mathrm{mg}$ of frozen liver tissue was homogenized for 30 seconds at $5000 \mathrm{rpm}$ in a closed tube with 1.0-mm glass beads and $1.0 \mathrm{ml}$ SET buffer (Sucrose $250 \mathrm{mmol} / \mathrm{l}$, EDTA $2 \mathrm{mmol} / \mathrm{l}$ and Tris 10 $\mathrm{mmol} / \mathrm{l})$. Complete cell destruction was done by 2 freeze-thaw cycles and 3 times passing through a 27-gauge syringe needle and a final freeze-thaw cycle. Protein content was measured using the BCA method Pierce, Rockford, IL). Liver cholesterol and triglyceride levels were quantified using 
cholesterol liquicolor kit [CHOD-PAP, Roche, Basel, Switzerland; triglycerides: GPOtrinder, TRO100, Sigma Aldrich, St. Louis, MO, USA)] following the manufacturer's protocol on a Benchmark 550 microplate reader (Bio-Rad, Hercules, CA, USA).

\subsection{Liver Histology}

Immunostainings were carried out on frozen liver sections $(7 \mu \mathrm{m})$ as described previously [23]. Briefly, cryosections were fixated in dry acetone followed by blocking with $\mathrm{H} 2 \mathrm{O} 2$ solution. Next, tissue sections were treated with avidin/biotin solution (Vector; SP2001) for 30 minutes followed by 1 hour incubation with primary antibody [infiltrated macrophages marker, Mac-1; MAB1124; clone M1/70; 1:500)], [F4/80;101201; Biolegio; (1:50)]. Sections later were incubated in secondary antibody (Rabbit anti- Rat IgG Biotin (6180-08), Southern Biotech, Birmingham, USA) which was detected using the Peroxidase Substrate kit AEC (Vector Laboratories, SK-4200, Peterborough, United Kingdom). Haematoxylin was used for nuclear counterstaining. Lipid and collagen content was assessed by using neutral lipid marker Oil Red O (ORO; O0625; Sigma-Aldrich) and Sirius red, (Direct red 80; 43664; Sigma-Aldrich) respectively according to standard protocol. Images were captured with a Nikon digital camera DMX1200 and ACT-1 v2.63 software (Nikon Instruments Europe, Amstelveen, The Netherlands).

\subsection{CTSD activity measurements}

CTSD activity was measured using the CTSD activity assay kit (MBL International, Woburn, MA) as described previously [16]. Briefly, cell medium was incubated with CTSD substrate and reaction buffer for 1 hour at $37^{\circ} \mathrm{C}$. Samples were measured using a fluorescence plate reader with a 328-nm excitation filter and 460-nm emission filter and CTSD activity is expressed as relative fluorescence units.

\subsection{FACS analysis}

Fluorescence activated cell sorting (FACS) analysis was performed as described previously [19]. Tail vein blood was collected from mice before the start of the treatment (TO) as well at the end of the treatment (T1). Using Trucount tubes (BD Biosciences, Breda, The Netherlands), staining was performed according to the manufacturer's instructions, to detect the monocyte population (NK1.1Ly6G-CD11b+; Ly6C). Briefly, heparinized blood samples were mixed and incubated for 10 minutes in the dark at room temperature (RT) with CD16/32 antibody (eBioscience, Halle-Zoersel, Belgium) to block Fc receptor. Samples were then gently vortexed with the appropriate antibodies (PE Mouse Anti-Mouse NK-1.1 (1:100); APC-Cy ${ }^{\text {TM7 }}$ Rat Anti-Mouse Ly-6G (1:100); Anti-Mouse Ly-6C-APC (1:10) (Miltenyi, Bergisch Gladbach, Germany) and incubated in the dark at RT for 20 minutes [24]. All antibodies were diluted in FACS buffer (PBS, 0.1\% BSA, 0.01\% sodium azide). Finally, samples were mixed and incubated in the dark at RT for 15 minutes with an RBClysis solution $(8.4 \mathrm{~g} \mathrm{NH} 4 \mathrm{Cl}+0.84 \mathrm{~g}$ $\mathrm{NAHCO} 3$ in 1-liter $\mathrm{H} 2 \mathrm{O}, \mathrm{pH}$ 7.2-7.4). Sample stainings were quantified using BD FACSCanto II flow cytometer (BD Biosciences). 


\subsection{Faecal bile acid measurements}

Faecal measurement was performed on faeces collected after 24 hours from individually caged mice. Total faecal bile acid levels were specifically determined as described previously [25].

\subsection{Label-free LC-MS (Liquid Chromatography Mass Spectrometry) based proteomics}

For proteomics experiments, liver homogenates were used ( $\mathrm{n}=3$ for each group of mice). The samples were prepared as described previously [26]. Briefly, proteins were extracted, reduced with $20 \mathrm{mM}$ dithiothreitol for 45 minutes and alkylated with $40 \mathrm{mM}$ iodoacetamide for 45 minutes in the dark. Alkylation was terminated using $20 \mathrm{mM}$ DDT which is followed by digestion with a mixture of LysC and trypsin at a ratio of 1:25 (enzyme:protein) at $37{ }^{\circ} \mathrm{C}$ overnight. The digestion was stopped by formic acid. Peptide separation was performed on a Thermo Scientific (Dionex) Ultimate 3000 Rapid Separation UHPLC system equipped with a PepSep C18 analytical column $(15 \mathrm{~cm}$, ID $75 \mu \mathrm{m}, 1,9 \mu \mathrm{m}$ Reprosil, $120 \AA ̊$ ). Peptide samples were first desalted on an online installed C18 trapping column. After desalting peptides were separated on the analytical column with a 90-minute linear gradient from $5 \%$ to $35 \%$ Acetonitrile (ACN) with $0.1 \% \mathrm{FA}$ at $300 \mathrm{~nL} / \mathrm{min}$ flow rate. The UHPLC system was coupled to a $Q$ Exactive $H F$ mass spectrometer (Thermo Scientific). DDA settings were as follows. Full MS scan between $250-1,250 \mathrm{~m} / \mathrm{z}$ at resolution of 120,000 followed by MS/MS scans of the top 15 most intense ions at a resolution of 15,000 .

For protein identification and quantification, DDA spectra were analyzed with Proteome Discoverer version 2.2. The search engine Sequest was used with the Swiss-Prot mouse database (Mus musculus, TaxID 10090). The settings for the database search were similar as before [25]. Proteins with a falsediscovery rate $<1 \%$ were considered for the analysis and were normalized to the total peptide amount. Statistical significance of changes observed in protein abundance was performed using ANOVA. The Benjamini- Hochberg method was used to correct $P$-values for multiple testing. Proteins were considered differently regulated if the fold change was $\geq 1.5(\log 2 \geq 0.58)$ and a $p$-value of $\leq 0.05$. The differentially regulated proteins were then imported to the EnrichR tool and KEGG database (Version 2019, mouse) was used to demonstrate the top 10 pathways of up or down-regulated proteins ranked by the combined score which is calculated by multiplying the unadjusted, rather than the adjusted, p-values with the z-scores [26].

\subsection{Statistical analyses}

Except for proteomics, rest of the data were analysed using GraphPad Prism software (GraphPad, San Diego, California, USA), version 6.0 for Windows. Experimental groups were compared using twotailed student's t-test or two-way ANOVA where appropriate or by one-way ANOVA followed by a Tukey post-hoc test. Data are expressed as mean \pm SEM and were considered significant at $p<0.05$, in which ${ }^{*} p<0.05 ; * * p<0.01$; or $* * * p<0.001$ respectively. 


\section{Results}

\subsection{Cathepsin D activity is reduced using low-dose extracellular CTSD inhibitor}

To evaluate their potency and specificity, both the intracellular and extracellular CTSD smallcompound inhibitors were tested using in vitro methods. CTSD activity was measured from the medium of bone marrow-derived macrophages (BMDMs) that were treated with either of the inhibitors. CTD-002 (i.e., the extracellular CTSD inhibitor) at a concentration of $100 \mu \mathrm{M}$ significantly blocked CTSD activity when compared to DMSO (vehicle) as measured from the supernatant of BMDMs. In contrast, the intracellular CTSD inhibitor, GA-12, had no effect on CTSD activity in the supernatant, thus confirming the selective targeting of respective inhibitors (Figure 1).

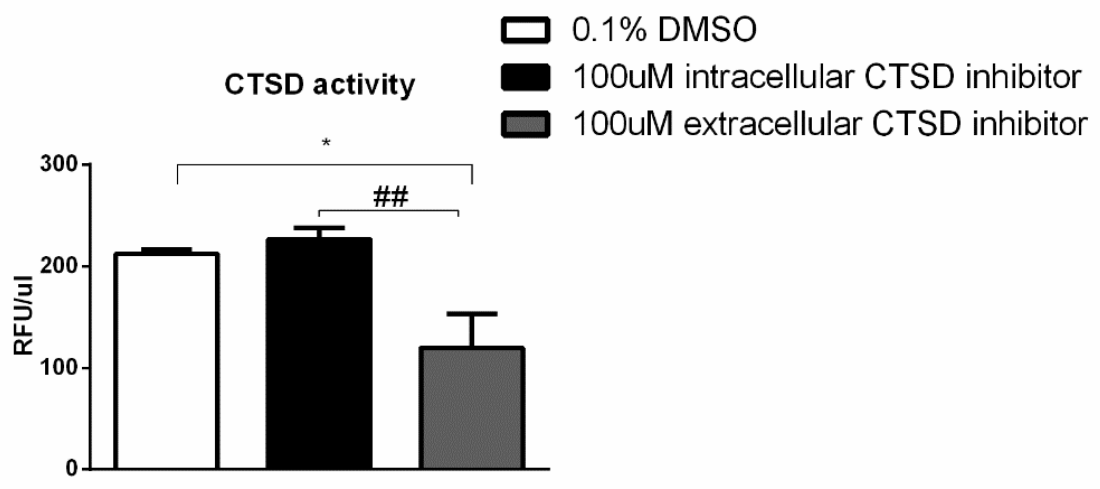

Figure 1 Efficacy of the cathepsin inhibitors. CTSD activity measurements from the supernatant of the Wt mouse BMDMs, exposed to 24 hours and subsequently treated with either vehicle control (0.1\% DMSO) or $100 \mu \mathrm{M}$ CTSD inhibitors for 4 hours. Error bars represent $\pm S E M ; n=5$ from two independent experiments. * represents $p<0.05$ compared to control mice. \#\# represents $p<0.01$ compared to intracellular CTSD inhibitor- treated mice by means of one-way ANOVA.

\subsection{Extracellular CTSD inhibition led to reduced hepatic lipid levels compared to intracellular CTSD inhibition}

To test the effects of CTSD inhibition in NASH-associated dyslipidemia, Ldlr-/- mice on HFC diet were injected with either control or intra- or extracellular CTSD inhibitors for a period of 10 weeks. The small-compound CTSD inhibitors showed no visible signs of toxicity in mice. Food intake, body morphology and growth of all mice stayed normal throughout the 10-week experimental period. No abnormalities were detected in any of the animals at autopsy. To investigate the effects of CTSD inhibition on lipid metabolism, hepatic and plasma lipid levels were measured. After ten weeks of HFC diet, both plasma and liver cholesterol and triglycerides were significantly elevated in HFC-fed mice compared to mice on a chow diet, confirming the effect of HFC on hepatic and plasma lipid levels (Figures S2A-D). Further, treating HFC-fed mice with either the intracellular or the extracellular CTSD inhibitor decreased hepatic cholesterol levels compared to control mice (Figure 2A). However, only upon administration of the extracellular CTSD inhibitor, a reduction in hepatic triglyceride was observed (Figure 2B). To confirm the hepatic changes in lipid content at the histological level, we 


\section{Chapter 4}

performed an oil red $\mathrm{O}$ staining which demonstrated elevated fat deposition in HFC mice compared to chow mice (Figure S2C). Moreover, in line with hepatic lipid measurements, extracellular CTSDinhibitor treated mice showed significant reduction of intracellular and near significant reduction of sinusoidal lipid deposition compared to HFC mice (Figures 2C, D) suggesting a clear reduction in hepatic lipid content upon extracellular CTSD inhibition. To further define the impact of extracellular CTSD inhibition on hepatic lipid metabolism, hepatic gene expression analysis using qPCR was performed. Compared to control and intracellular CTSD inhibitor-treated mice, inhibition of extracellular CTSD led to increased hepatic expression of cytochrome P450 7A1 (Cyp7a1), a ratelimiting enzyme in the breakdown of cholesterol to bile acids, suggesting increased cholesterol conversion only upon extracellular CTSD inhibition (Figure 2E). No pronounced effects in other lipid metabolism- related genes were observed (Figure S4A). However, plasma lipid levels remained similar among all experimental groups (Figure 2F). Altogether, these findings demonstrate that extracellular, rather than intracellular, CTSD inhibition decreases hepatic lipid accumulation in a mouse model for NASH. 
(A)

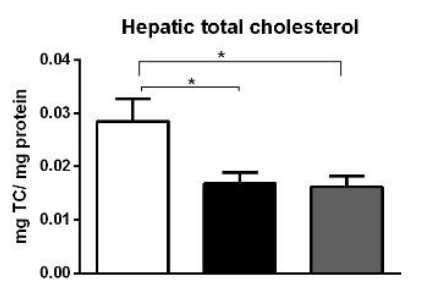

(C)
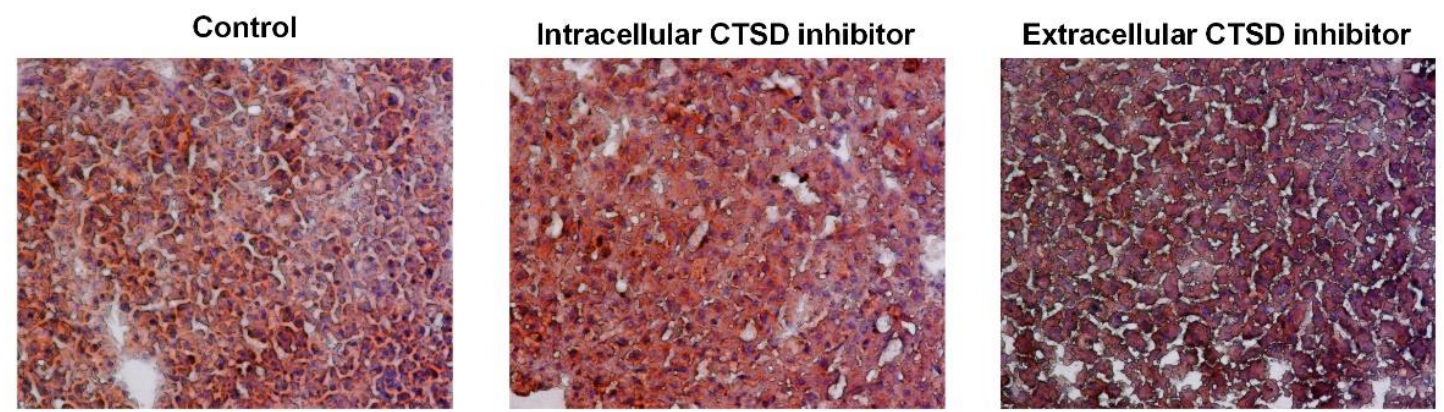

(D)
(B)

Hepatic total triglycerides

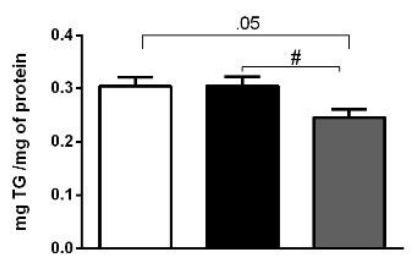

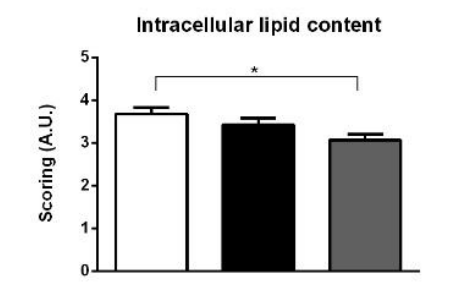

(F)

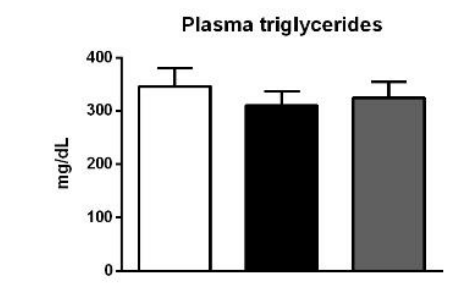

\section{)}

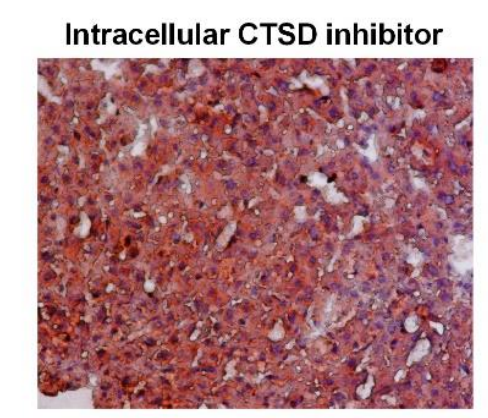

(E)
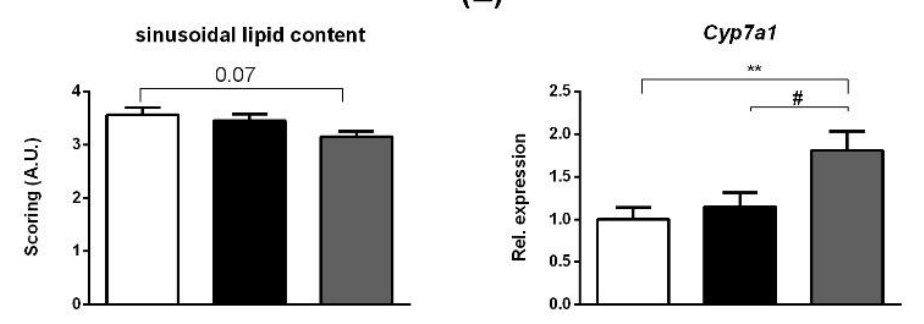

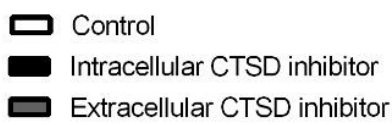

Extracellular CTSD inhibitor

Figure 2 Hepatic and plasma lipids in hyperlipidaemic LdIr-/- mice with or without CTSD inhibition: (A, B) Total hepatic cholesterol and triglyceride levels. (C) Representative pictures of oil red o staining on frozen liver sections (original magnification, 200x). (D) Scoring of hepatic lipid content of oil red o staining using arbitrary units (A.U.) (E) Hepatic gene expression analysis of Cyp7a1. (F) Plasma triglyceride and cholesterol measurements. Error bars represent $\pm S E M ; n=16-20$ per each group; * represents $p<0.05$ and ${ }^{* *}$ represents $p<0.01$ compared to control mice. \# represents $p<0.05$ compared to intracellular CTSD inhibitor- treated mice by means of one-way ANOVA. 


\subsection{Extracellular CTSD inhibition led to increased faecal bile acid levels compared to intracellular CTSD inhibition}

To confirm the increased conversion of cholesterol into bile acid precursors, we opted to further investigate bile acid metabolism by assessing faecal bile acid levels. Faecal levels of primary bile acids, chenodeoxycholic acid (CDCA) and ursodeoxycholic acid (UDCA) were significantly enhanced in the extracellular inhibitor-treated group as compared to mice that received the intracellular inhibitor, indicating increased cholesterol breakdown in the former mice (Figure 3A). In line, secondary bile acids including $\alpha$-muricholic acid and $\beta$-muricholic acid showed increased faecal levels in extracellular inhibitor-treated mice as compared to intracellular inhibitor-treated mice (Figure 3B). No differences in the faecal bile acid levels were observed between control and extracellular inhibitor-treated mice. Collectively, our data suggest that inhibition of extracellular CTSD activity in NASH mice results in increased disposal of cholesterol via increased conversion into bile acids leading to their subsequent excretion.

(A)

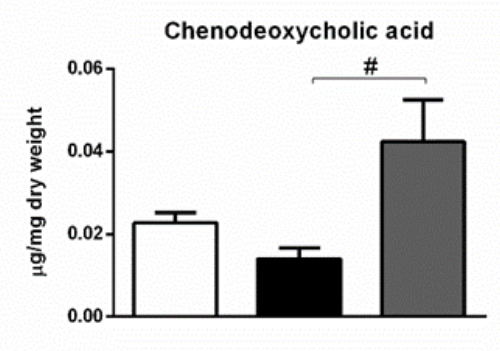

(B)

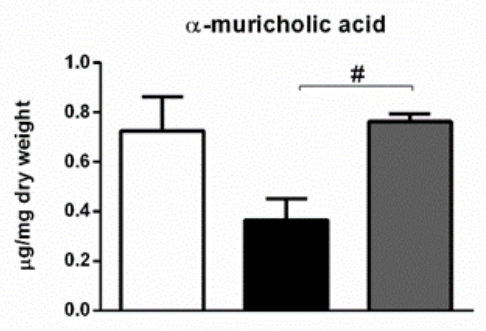

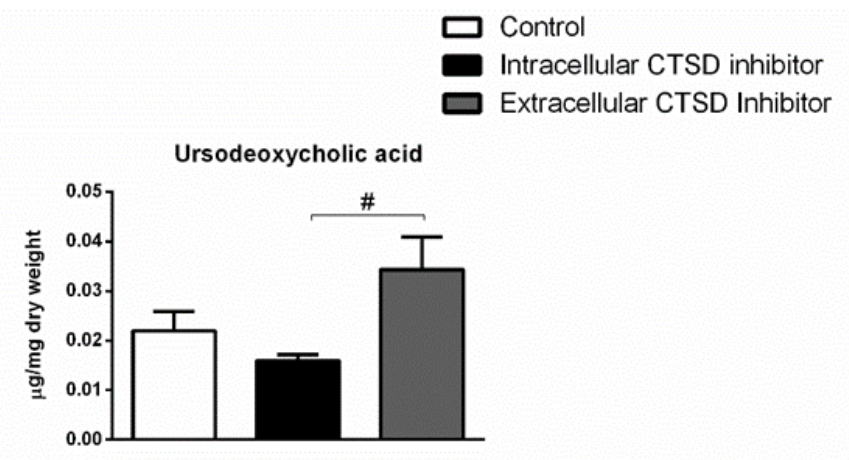

$\beta$-mucricholic acid

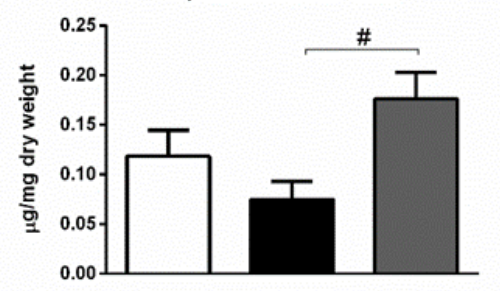

Figure 3 Increased faecal bile acid levels upon extracellular CTSD inhibition in LdIr-/- mice. (A) Faecal measurements of primary bile acids (B) Secondary bile acids in the faeces. Error bars represent \pm SEM. $n=4$ per group. \# represents $p<0.05$ compared to intracellular CTSD inhibitor-treated mice as determined by one-way ANOVA.

\subsection{Extracellular CTSD inhibition led to reduced systemic inflammation compared to intracellular CTSD inhibition}

Next, we examined the role of extracellular CTSD on inflammation at both hepatic and systemic levels. Firstly, inflammatory status was significantly elevated in HFC-fed mice compared to chow mice both at systemic and hepatic levels (Figures $\mathrm{S} 2 \mathrm{E}-\mathrm{G}$ ). To explore the status of systemic inflammation 
upon extracellular CTSD inhibition, we analysed the profile of circulating monocytes using fluorescence-activated cell sorting (FACS). However, no statistical differences were observed in the number of total and pro- inflammatory monocytes (DT1-T0) between three different groups of HFC mice (Figures 4A, B). Further, indicating the decrease of systemic inflammation upon extracellular CTSD inhibition, extracellular inhibitor-treated mice showed a significant increase in the total number of anti-inflammatory monocytes (Ly6c-) compared to the control mice and intracellular CTSD inhibitor- treated mice (near significant; $p=0.07$ ) (Figure 4C). However, no significant changes in Tcell population were observed upon extracellular CTSD inhibition (Figures 4D-F). Together, these data demonstrate minor reduction in systemic inflammation upon extracellular CTSD inhibition. Next to systemic inflammation, the effects of extracellular CTSD inhibition on hepatic inflammation were assessed by hepatic immunostainings for the inflammatory cell markers Mac- 1 and F4/80. The levels of infiltrated macrophages and $\mathrm{F} 4 / 80$ positive macrophage remained unaffected upon extracellular CTSD inhibition (Figures 4G-J). Lastly, as expected, the extent of hepatic fibrosis, an advanced NASH feature was no different in chow and experimental groups of HFC as demonstrated by Sirius red staining (Figure S3). 
(A)

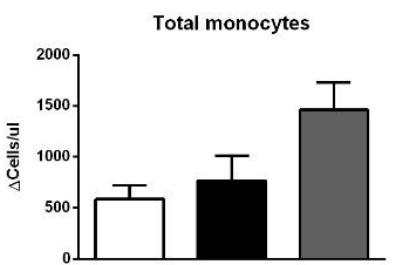

(C)

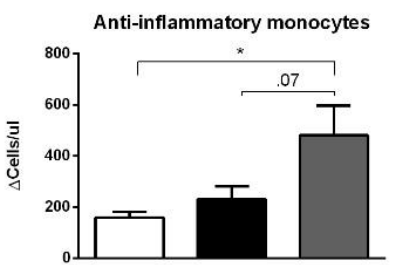

(F)

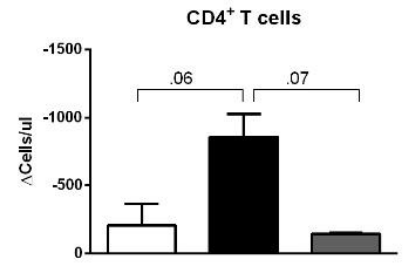

(I)

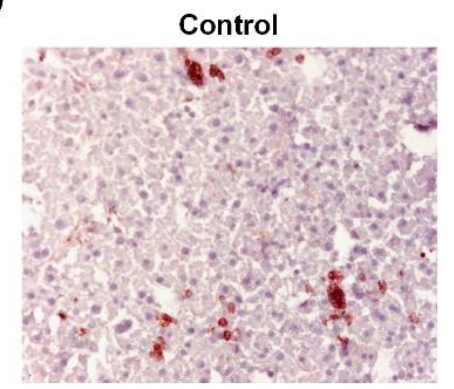

(J)

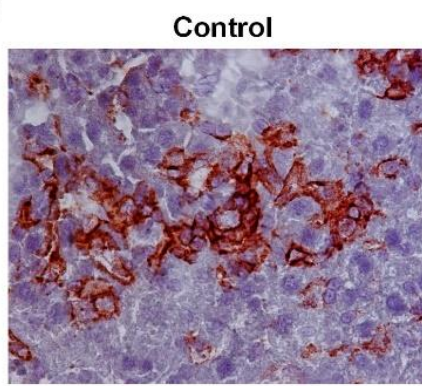

(B)

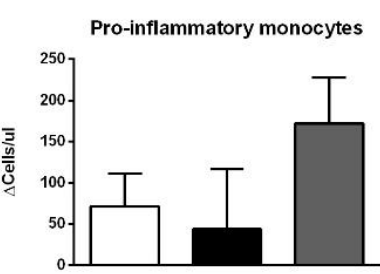

(D)

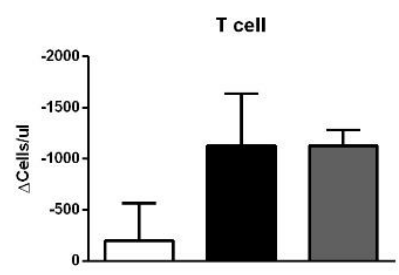

(G)

(E)

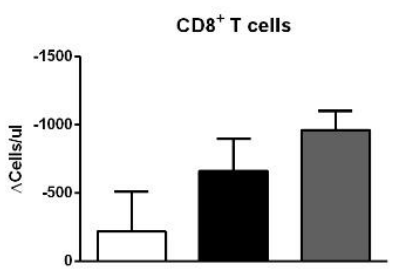

(H)
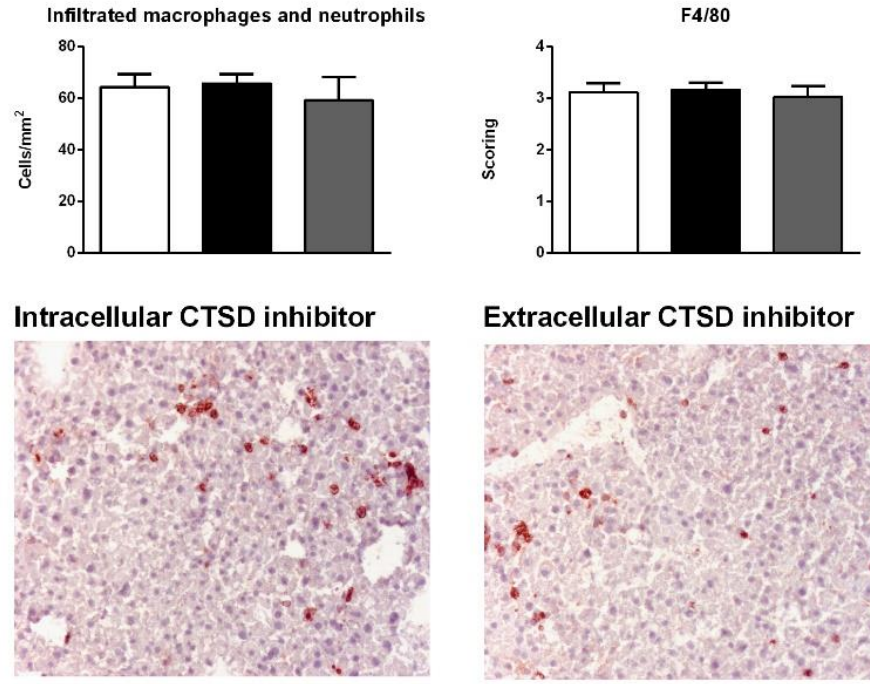

Extracellular CTSD inhibitor
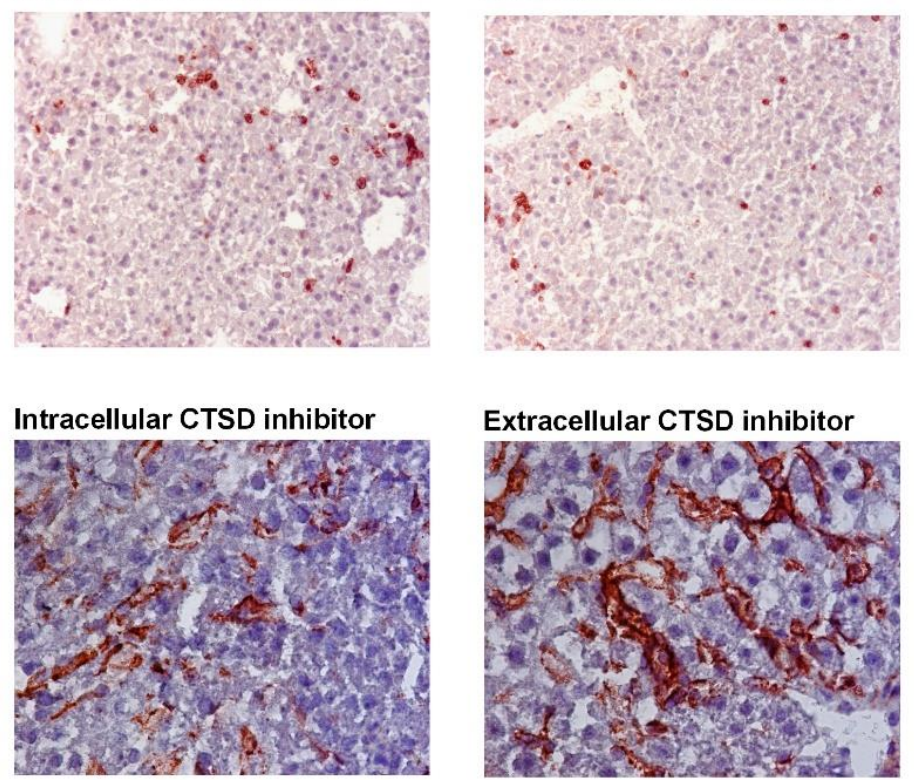

口 Control

Intracellular CTSD inhibitor

Extracellular CTSD inhibitor
Extracellular CTSD inhibitor

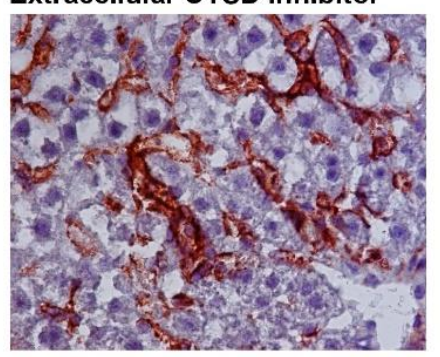

Figure 4 Reduction in inflammatory status upon extracellular CTSD inhibition in Ldlr-/- mice: (A-F) Total number of monocyte and T- cell population as measured by FACS. (G) Liver sections were stained for infiltrating macrophages and neutrophil cells (Mac-1). Positive cells were counted in six microscopical views. (H) Immunostaining of livers for F4/80. Positive cells were scored assessed and given a score in arbitrary units (A.U.) (I) Representative pictures of Mac-1 staining (original magnification, 200x) and (J) F4/80 (original magnification, 400x). Error bars represent SEM; $n=5$ per each group for FACS and $n=3$ per group for proteomics; * represents $p<0.05$ compared to control mice as determined by one-way ANOVA. 


\subsection{Label-free quantitative proteomics and pathway enrichment analysis}

To obtain a comprehensive understanding of the proteomic changes upon extracellular and intracellular CTSD inhibition in the liver, label-free quantitative proteomics was performed. After data processing, a total of 1905 proteins were identified. The number of significantly differentially regulated proteins were plotted using volcano plots (Figure S5). The complete list of significantly different regulated proteins ( $\log 2 \geq 0.58)$ are mentioned in Tables S1-S6.

To further understand the functions of these differentially regulated proteins, enrichment analysis was performed to identify specific pathways. The top 10 KEGG (Kyoto encyclopaedia of genes and genomes) pathways that are most significantly enriched are displayed in Table 1. Compared to control and intracellular inhibitor-treated mice, extracellular inhibitor-treated mice showed enrichment of pathways related to lipid metabolism. Linoleic acid metabolism, steroid hormone biosynthesis, fatty acid biosynthesis and elongation were significantly enriched upon extracellular CTSD inhibition, suggesting improved lipid metabolism in these mice. Moreover, retinol metabolism, PPAR signalling, taurine/ hypotaurine metabolism and caffeine metabolism were upregulated in extracellular inhibitor- treated mice (Table 1A, B). In addition to lipid-related pathways, ferroptosis, mineral absorption, quinone biosynthesis, ascorbate and aldarate metabolism, NAFLD and chemical carcinogenesis pathways were also enriched upon extracellular CTSD inhibition. Among the downregulated pathways in extracellular inhibitor-treated group,

IL-17 pathway (proteins S100A8/A9), African trypanosomiasis, malaria (haemoglobin subunit beta 1), arginine and proline metabolism (creatinine kinase M-type) and glycosaminoglycan biosynthesis pathways (Xylosyltransferase 2) were in top 5 pathways compared to control (Table $1 \mathrm{C}$ ). Interestingly, protein S100A8/A9, which is a ligand complex for Toll-like receptor 4 (TLR-4), was the most significantly downregulated protein suggesting that extracellular CTSD inhibition potentially reduces inflammation in a TLR-4 dependent manner. Moreover, pathways related to cardiac function (hypertrophic and dilated cardiomyopathy, cardiac muscle function), glycolysis, metabolic pathways (glycine, serine, threonine, thiamine, pyruvate, propanoate metabolism) and glucagon signalling were decreased in extracellular inhibitor-treated mice compared to intracellular CTSD inhibitortreated mice (Table 1D).

Most importantly, intracellular inhibitor-treated mice showed downregulation of proteins involved in mitochondrial oxidative phosphorylation and electron transport chain compared to control and extracellular inhibitor-treated mice (Tables S1 and S4). According to KEGG pathways, intracellular inhibitor-treated mice showed enrichment of pathways belonging to muscle contraction and glycolysis compared to control (Table S7). Furthermore, proteins that are involved in hepatic stellate cell activation such as tropomyosin alpha-1 and beta chain, myosin regulatory light chain 2 and myosin 7 were upregulated. Taken together, these findings demonstrate that extracellular CTSD fraction regulates distinct pathways compared to intracellular CTSD in the context of NASH. 
Table 1: Differentially regulated proteins were imported to EnirchR tool and KEGG database was used for pathway enrichment analysis. Top 10 enriched pathways ranked by combined score are listed below.

(A) KEGG database (Mouse) - extracellular inhibitor-treated mice/ control

\begin{tabular}{ccc}
\hline Name & P-value (p) & Combined Score \\
\hline Caffeine metabolism & 0.005388 & 967.31 \\
\hline Linoleic acid metabolism & 0.00001168 & 757.16 \\
\hline Biosynthesis of unsaturated fatty acids & 0.0003734 & 548.11 \\
\hline Taurine and hypotaurine metabolism & 0.009858 & 466.61 \\
\hline Ferroptosis & 0.0005847 & 413.57 \\
\hline Mineral absorption & 0.0007077 & 366.34 \\
\hline PPAR signaling pathway & 0.002613 & 155.48 \\
\hline Steroid hormone biosynthesis & 0.002860 & 146.24 \\
\hline Retinol metabolism & 0.002988 & 141.96 \\
\hline Fatty acid elongation & 0.02579 & 140.14 \\
\hline
\end{tabular}

(B) KEGG database (Mouse) -extracellular inhibitor-treated mice/ intracellular inhibitor-treated mice

\begin{tabular}{ccc}
\hline Name & P-value (p) & Combined Score \\
\hline Steroid hormone biosynthesis & $4,59 \mathrm{E}-12$ & 824.33 \\
\hline Retinol metabolism & $5,43 \mathrm{E}-09$ & 535.98 \\
\hline Linoleic acid metabolism & $1,59 \mathrm{E}-05$ & 429.79 \\
\hline Chemical carcinogenesis & $1,90 \mathrm{E}-07$ & 407.05 \\
\hline Fatty acid biosynthesis & 0.00006776 & 351.63 \\
\hline Peroxisome & $6,11 \mathrm{E}-04$ & 203.82 \\
\hline Ubiquinone and other terpenoid-quinone biosynthesis & 0.001803 & 196.37 \\
\hline Non-alcoholic fatty liver disease (NAFLD) & $2,59 \mathrm{E}-04$ & 154.53 \\
\hline Ascorbate and aldarate metabolism & 0.0005151 & 143.80 \\
\hline PPAR signaling pathway & 0.00001057 & 138.25 \\
\hline
\end{tabular}

(C) KEGG database (Mouse) -control/ extracellular inhibitor-treated mice

\begin{tabular}{ccc}
\hline Name & P-value (p) & Combined Score \\
\hline IL-17 signaling pathway & 0.002357 & 166.22 \\
\hline African trypanosomiasis & 0.03076 & 111.59 \\
\hline Malaria & 0.03850 & 83.09 \\
\hline Arginine and proline metabolism & 0.03927 & 80.93 \\
\hline Glycosaminoglycan biosynthesis & 0.04158 & 75.00 \\
\hline Glutathione metabolism & 0.05001 & 58.51 \\
\hline Metabolism of xenobiotics by cytochrome P450 & 0.05153 & 56.17 \\
\hline PPAR signaling pathway & 0.06590 & 39.99 \\
\hline Protein digestion and absorption & 0.06965 & 37.00 \\
\hline Chemical carcinogenesis & 0.07263 & 34.87 \\
\hline
\end{tabular}


(D) KEGG database (Mouse) -intracellular inhibitor-treated mice/ extracellular inhibitor-treated mice

\begin{tabular}{ccc}
\hline Name & P-value (p) & Combined Score \\
\hline Glycolysis / Gluconeogenesis & 0.00004577 & 426.09 \\
\hline Hypertrophic cardiomyopathy (HCM) & 0.00009653 & 307.16 \\
\hline Dilated cardiomyopathy (DCM) & 0.0001105 & 289.22 \\
\hline Thiamine metabolism & 0.01564 & 263.99 \\
\hline Cardiac muscle contraction & 0.003005 & 141.82 \\
\hline Propanoate metabolism & 0.03207 & 105.68 \\
\hline Glucagon signaling pathway & 0.005077 & 98.65 \\
\hline Pyruvate metabolism & 0.03917 & 81.20 \\
\hline Glycine, serine and threonine metabolism & 0.04119 & 75.94 \\
\hline Adrenergic signaling in cardiomyocytes & 0.01042 & 58.74 \\
\hline
\end{tabular}

\subsection{Anti-Inflammatory Effects of Extracellular CTSD Inhibitor Are LPS-Dependent}

To better understand the link between extracellular CTSD and TLR-4 mediated inflammation, we investigated whether lipopolysaccharide (LPS), a TLR4 agonist, influences the inflammatory effects of extracellular CTSD inhibitor. OxLDL- stimulated mouse bone-marrow derived macrophages were treated with extracellular CTSD inhibitor in the presence or absence of LPS. Only in the presence of LPS, extracellular CTSD inhibitor reduced gene expression levels of inflammatory cytokines, Tnfa and $\mathrm{Ccl} 2$ (Figures 5A, B) suggesting that the anti-inflammatory effects of extracellular CTSD inhibitor are TLR-4 dependent.

(A)

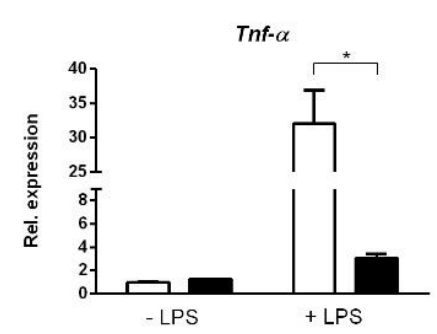

(B)

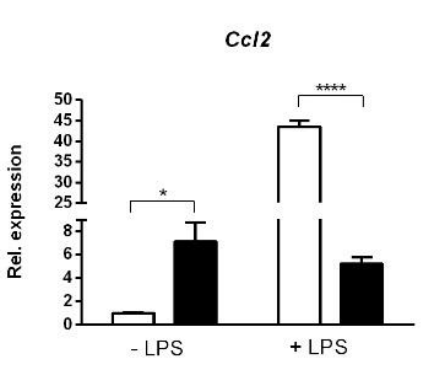

Figure 5 Anti-inflammatory effects of extracellular CTSD inhibitor are LPS- dependent: (A, B) Gene expression levels of Tnfa, and $C c / 2$ from mouse Wt mouse BMDMs. Gene expression data are shown relative to DMSO-treated BMDMs with no LPS. Error bars represent $\pm S E M ; n=3$ from an independent experiment. * represents $p<0.05$ and $* * * *$ represents $p<0.001$ compared to DMSO-treated BMDMs as determined by two-way ANOVA.

\section{Discussion}

Except for lifestyle interventions such as caloric restriction and exercise, there are currently no welldefined approved therapeutic strategies for NASH. As lifestyle changes are often insufficient, novel and safer pharmacologic treatments for NASH are urgently needed. While inhibition of CTSD activity was proven beneficial for NASH, the specific mechanisms by which extracellular and intracellular fractions of CTSD affect NASH were not explored. In the current study, we have identified that 
extracellular CTSD inhibition has more beneficial and less toxic effects in reducing metabolic inflammation over intracellular CTSD. These findings demonstrate the potential of extracellular CTSD to serve as a promising and safe target for the treatment of NASH.

Intracellular CTSD activity is accountable for cellular processes such as breakdown of intracellular proteins [28], cell signalling [29] and apoptosis [30]. Therefore, CTSD knockout mice develop intestinal necrosis, destruction of lymphoid cells and myelin sheath atrophy leading to early death, suggesting CTSD as a key protein in physiology [21]. In line, our proteomics data showed that inhibition of intracellular CTSD led to predominant downregulation of proteins specifically involved in mitochondrial oxidative phosphorylation and electron transport function namely Cox4i1, Cox6c, NADH dehydrogenase as well as CYP class of enzymes (Cyp2c29, Cyp3a41a, Cyp3a11, Cyp2c40, Cyp2c37, Cyp1a2, Cyp2e1) as found in 4/10 KEGG enriched pathways. It must be noted that downregulation of hepatic CYP enzymes might result in elevated plasma drug levels, adverse drug effects or drug-induced toxicity and inflammation [31] indicating unfavourable effects of intracellular CTSD inhibition. Besides executing its classical homeostatic activity inside the cells, CTSD also functions in the extracellular space both in physiological and pathological conditions [32]. While controlled proteolytic activity of extracellular cathepsin $D$ is evidently important for vital processes such as extracellular matrix (ECM) degradation during tissue repair, uncontrolled CTSD expression and activity is more commonly observed under pathological conditions [33, 34]. Specifically, excessive extracellular CTSD activity has been implicated in metabolic inflammatory conditions [18, $22,35,36]$. In line with these compelling findings, we have demonstrated that extracellular CTSD activity is associated with NASH pathogenesis. Given evidence for the site-specific role of CTSD in pathophysiological processes, targeted blocking of its activity is critical to prevent any off-target side effects [37]. To this end, selective effects of respective small-compound CTSD inhibitors obtained in our in vitro experiment strengthens the possibility that these inhibitors may have therapeutic value in treating NASH without causing disturbances of generalized lysosomal CTSD operations.

In our current study, we found pronounced effects of CTSD inhibition in the liver such as reduced lipid levels and alterations in metabolic pathways amongst others. This observation is in line with the concept that complex interplay between lipid acquisition and lipid disposal occurs in NASH [38]. Lipids undergo constant recycling and relocation in the cell and lysosomes play an important role in this recycling as they regulate hepatic lipid catabolism and mobilization [39]. In agreement, lysosomal dysfunction accompanied by aberrant cathepsin expression and activity is shown to be involved with the onset of NASH [16, 40-42]. Similar to these observations, we have previously shown that CTSD plays a central role in lipid metabolism during NASH and that its inhibition reduces hepatic steatosis by biliary cholesterol elimination [19]. The present study confirms the findings of this earlier report and provides insight for the specific role of extracellular CTSD fraction in lipid metabolism. In this study, pathways involved in hepatic lipid metabolism such as linoleic acid, fatty acid and steroid hormone biosynthesis have been found enriched in extracellular inhibitor- treated mice. The increased lipid biosynthesis upon extracellular CTSD inhibition likely reflects a compensatory response to the reduction in hepatic lipid levels and enhancement of cholesterol clearance. Further, amino acid [glycine, serine, and threonine] and pyruvate metabolism involving L-lactate dehydrogenase, enolase, and phosphoglycerate mutase proteins were downregulated suggesting reduced hepatic gluconeogenesis to compensate for the energy excess in the hyperlipidaemic mice. In addition to lipid-related pathways, proteomics analysis revealed enrichment of taurine and hypotaurine metabolism, retinol metabolism and PPAR signalling pathways upon extracellular CTSD 
inhibition. Taurine is an amino acid that is known to have diverse protective effects in liver diseases by suppressing diet- induced steatosis and inflammation among others [43]. Noteworthy, retinoic acid and PPAR are known to synergically reverse liver fibrosis [44, 45]. These findings further reinforce the potential benefits of extracellular CTSD inhibition in the context of liver diseases.

Furthermore, KEGG analysis revealed that IL-17 signalling pathway, drug and glutathione metabolism were downregulated upon extracellular CTSD inhibition. Relevantly, IL-17 signalling has been demonstrated to be involved in the pathogenesis of NASH. IL-17 pathway revealed the downregulation of S100A8/9 proteins. S100A8/9 proteins act as a ligand for Toll-like receptor 4 (TLR4) and are known to be released extracellularly, where they amplify inflammatory responses by inducing cytokine production such as IL-17 $[46,47]$. Moreover, TLR-4 activation is required for IL17-mediated inflammation [48]. Furthermore, both broad and selective cathepsin inhibitors are known to suppress IL-17 producing T helper cells during inflammatory process [49]. Intriguingly, TLR4-dependent signalling is known to drive extracellular catabolism of modified lipoproteins by lysosomal proteases [50]. These findings suggest that extracellular CTSD released upon hyperlipidaemic conditions might interact with S100A8/9 complex to activate the TLR4 signalling pathway which further exacerbates inflammation in a positive feedback mechanism.

It should however be taken into account that we could not detect profound effects of extracellular CTSD inhibition on other markers of hepatic inflammation. Moreover, while our data suggest an improvement in systemic inflammation, these data were retrieved from only a limited number of mice $(n=5)$. More in-depth analysis including additional mice is therefore essential to confirm our findings. Further, 16 weeks of dietary intervention didn't reveal significant differences in plasma inflammatory markers in low and HFC fed Ldlr-/- mice [51], suggesting that longer periods of diet are required to see such changes. As such the results of the current study are not sufficient to conclude that extracellular CTSD has major effects on reducing inflammation status. Therefore, it is currently unclear whether the dose of $50 \mathrm{mg} / \mathrm{kg}$ of inhibitor and 10 weeks of HFC diet are sufficient to interfere with the inflammatory network. Future studies with higher and/or more frequent injections of CTSD inhibitor are warranted to answer these questions. Furthermore, it remains to be determined to what extent these potential mechanisms take place in humans.

Recently, we showed that extracellular CTSD inhibition led to reduced hepatic steatosis and insulin sensitivity in rats [22]. However, it is noteworthy that the translational potential of these findings to humans is limited due to substantial differences in lipoprotein metabolism between humans and rats [52]. For instance, hyperlipidaemic rats are known to display elevated plasma levels of high-density lipoprotein cholesterol (HDL-c) and reduced low-density lipoprotein cholesterol (LDL-c) levels, while humans show reversed plasma lipoprotein profiles, with higher levels of LDL-c and lower HDL-c. Relevantly, knocking out the LDL receptor in mice is known to block the uptake of LDL and results in a phenotype with excessive plasma LDL-c levels. Further, Ldlr-/- mice have the largest amount of the cholesterol in the form of IDL/ LDL fraction [intermediate density lipoprotein/low-density lipoprotein] as seen in humans [53]. Thus, Ldlr-/- mice possess the plasma lipoprotein profile as comparable to humans and serve as a better model to study the "human-like" profile [54]. In agreement, Ldlr-/- mice have been used extensively as a research model to investigate pathways involved in human NASH [55-57]. The current study establishes a key role for extracellular CTSD in $\mathrm{NASH}$-induced dyslipidaemia. Despite these significant findings, there are certain limitations to our study that include no advanced inflammatory data and absence of human data with CTSD inhibitors. 


\section{Chapter 4}

Therefore, future studies using human models are warranted to expand our findings on inflammation and their validation in the clinical setting.

In conclusion, in contrast to the intracellular fraction of CTSD, extracellular CTSD inhibition has substantial benefits in restoration of lipid metabolism. Although the results of the current study should be interpreted with caution given the mild changes in inflammation, our data highlight that extracellular CTSD inhibition has favourable effects compared to intracellular inhibition and thus holds potential translational value as observed in a NASH mouse model with a 'human-like' lipoprotein profile. 


\section{References}

1. Younossi ZM, Koenig AB, Abdelatif D, Fazel Y, Henry L, Wymer M. Global Epidemiology of Nonalcoholic Fatty Liver Disease-Meta-Analytic Assessment of Prevalence, Incidence, and Outcomes. Hepatology (2016) 64(1):73-84. doi: 10.1002/hep.28431

2. Manne V, Handa P, Kowdley KV. Pathophysiology of Nonalcoholic Fatty Liver Disease/Nonalcoholic Steatohepatitis. Clinics Liver Dis (2018) 22(1):23-+. doi: 10.1016/j.cld.2017.08.007

3. Friedman SL, Neuschwander-Tetri BA, Rinella M, Sanyal AJ. Mechanisms of NAFLD Development and Therapeutic Strategies. Nat Med (2018) 24(7):908-22. doi: 10.1038/s41591-018-0104-9

4. Alkhouri N. Nonalcoholic Fatty Liver Disease (NAFLD) and Nonalcoholic Steatohepatitis (NASH): Prevalence, Therapeutic Innovations, and Stumbling Blocks for Clinical Development. Expert Opin Investig Drugs (2020) 29 (2):115-6. doi: 10.1080/13543784.2020.1721167

5. Takahashi Y, Sugimoto K, Inui H, Fukusato T. Current Pharmacological Therapies for Nonalcoholic Fatty Liver Disease/Nonalcoholic Steatohepatitis. World J Gastroenterol (2015) 21(13):3777-85. doi: 10.3748/wjg.v21.i13.3777

6. Sheka AC, Adeyi O, Thompson J, Hameed B, Crawford PA, Ikramuddin S. Nonalcoholic Steatohepatitis: A Review. JAMA (2020) 323(12):1175-83. doi: 10.1001/jama.2020.2298

7. Bieghs V, Hendrikx T, van Gorp PJ, Verheyen F, Guichot YD, Walenbergh SM, et al. The Cholesterol Derivative 27Hydroxycholesterol Reduces Steatohepatitis in Mice. Gastroenterology (2013) 144(1):167-78.e1. doi: 10.1053/j.gastro.2012.09.062

8. Houben T, Oligschlaeger Y, Bitorina AV, Hendrikx T, Walenbergh SMA, Lenders MH, et al. Blood-Derived Macrophages Prone to Accumulate Lysosomal Lipids Trigger ox LDL-Dependent Murine Hepatic Inflammation. Sci Rep (2017) 7(1):12550. doi: 10.1038/s41598-017-13058-z

9. Settembre C, Di Malta C, Polito VA, Garcia Arencibia M, Vetrini F, Erdin S, et al. TFEB Links Autophagy to Lysosomal Biogenesis. Science (2011) 332 (6036):1429-33. doi: 10.1126/science.1204592

10. Rosenfeld MG, Kreibich G, Popov D, Kato K, Sabatini DD. Biosynthesis of Lysosomal Hydrolases: Their Synthesis in Bound Polysomes and the Role of Co- and Post-Translational Processing in Determining Their Subcellular Distribution. J Cell Biol (1982) 93(1):135-43. doi: 10.1083/jcb.93.1.135

11. Ungewickell AJ, Majerus PW. Increased Levels of Plasma Lysosomal Enzymes in Patients With Lowe Syndrome. Proc Natl Acad Sci U S A (1999) 96 (23):13342-4. doi: 10.1073/pnas.96.23.13342

12. Hultberg B, Isaksson A, Sjöblad S, Ockerman PA. Acid Hydrolases in Serum From Patients With Lysosomal Disorders. Clin Chim Acta (1980) 100(1):33- 8. doi: 10.1016/0009-8981(80)90182-5

13. Hughes CS, Colhoun LM, Bains BK, Kilgour JD, Burden RE, Burrows JF, et al. Extracellular Cathepsin S and Intracellular Caspase 1 Activation are Surrogate Biomarkers of Particulate-Induced Lysosomal Disruption in Macrophages. Part Fibre Toxicol (2016) 13:19. doi: 10.1186/s12989-016-0129-5

14. Vidak E, et al. Cysteine Cathepsins and Their Extracellular Roles: Shaping the Microenvironment. Cells (2019) 8(3). doi: 10.3390/cells8030264

15. Hasilik A, Neufeld EF. Biosynthesis of Lysosomal Enzymes in Fibroblasts. Synthesis as Precursors of Higher Molecular Weight. J Biol Chem (1980) 255 (10):4937-45. doi: 10.1016/S0021-9258(19)85585-0

16. Walenbergh SM, Houben T, Rensen SS, Bieghs V, Hendrikx T, van Gorp PJ, et al. Plasma Cathepsin D Correlates With Histological Classifications of Fatty Liver Disease in Adults and Responds to Intervention. Sci Rep (2016) 6:38278. doi: 10.1038/srep38278

17. Moallem SA, Nazemian F, Eliasi S, Alamdaran SA, Shamsara J, Mohammadpour AH. Correlation Between Cathepsin D Serum Concentration and Carotid Intima- Media Thickness in Hemodialysis Patients. Int Urol Nephrol (2011) 43(3):841-8. doi: 10.1007/s11255-010-9729-4

18. Ding L, Goossens GH, Oligschlaeger Y, Houben T, Blaak EE, Shiri-Sverdlov R. Plasma Cathepsin D Activity Is Negatively Associated With Hepatic Insulin Sensitivity in Overweight and Obese Humans. Diabetologia (2020) 63(2):374-84. doi: 10.1007/s00125-019-05025-2

19. Houben T, Oligschlaeger Y, Hendrikx T, Bitorina AV, Walenbergh SMA, van Gorp PJ. Cathepsin D Regulates Lipid Metabolism in Murine Steatohepatitis. Sci Rep (2017) 7(1):3494. doi: 10.1038/s41598-017-03796-5

20. Ruan H, Hao S, Young P, Zhang H. Targeting Cathepsin B for Cancer Therapies. Horiz Cancer Res (2015) 56:23-40. 


\section{Chapter 4}

21. Guo DZ, Xiao L, Liu YJ, Shen C, Lou HF, Lv Y. Cathepsin D Deficiency Delays Central Nervous System Myelination by Inhibiting Proteolipid Protein Trafficking From Late Endosome/Lysosome to Plasma Membrane. Exp Mol Med (2018) 50(3):e457. doi: 10.1038/emm.2017.291

22. Khurana P, Yadati T, Goyal S, Dolas A, Houben T, Oligschlaeger Y, et al. Inhibiting Extracellular Cathepsin D Reduces Hepatic Steatosis in Sprague(-) Dawley Rats (Dagger). Biomolecules (2019) 9(5). doi: 10.3390/biom9050171

23. Jeurissen ML, Walenbergh SM, Houben T, Hendrikx T, Li J, Oligschlaeger Y, et al. Myeloid DLL4 Does Not Contribute to the Pathogenesis of Non- Alcoholic Steatohepatitis in Ldlr-/- Mice. PLoS One (2016) 11(11):e0167199. doi: 10.1371/journal.pone.0167199

24. Magro Dos Reis I, Houben T, Oligschläger Y, Bücken L, Steinbusch H, Cassiman D, et al. Dietary Plant Stanol Ester Supplementation Reduces Peripheral Symptoms in a Mouse Model of Niemann-Pick Type C1 Disease. J Lipid Res (2020) 61(6):830-9. doi: 10.1194/jlr.RA120000632

25. Czubayko F, Beumers B, Lammsfuss S, Lütjohann D, von Bergmann K. A Simplified Micro-Method for Quantification of Fecal Excretion of Neutral and Acidic Sterols for Outpatient Studies in Humans. J Lipid Res (1991) 32 (11):1861-7. doi: 10.1016/S0022-2275(20)41640-2

26. Rosenbrand R, Barata D, Sutthavas $P$, Mohren R, Cillero-Pastor B, Habibovic $P$, et al. Lipid Surface Modifications Increase Mesoporous Silica Nanoparticle Labeling Properties in Mesenchymal Stem Cells. Int J Nanomedicine (2018) 13:7711-25. doi: 10.2147/IJN.S182428

27. Chen EY, Tan CM, Kou Y, Duan Q, Wang Z, Meirelles GV, et al. Enrichr: Interactive and Collaborative HTML5 Gene List Enrichment Analysis Tool. BMC Bioinf (2013) 14:128. doi: 10.1186/1471-2105-14-128

28. Dubey V, Luqman S. Cathepsin D as a Promising Target for the Discovery of Novel Anticancer Agents. Curr Cancer Drug Targets (2017) 17(5):404-22. doi: 10.2174/1568009616666161229145115

29. Chapman HA. Cathepsins as Transcriptional Activators? Dev Cell (2004) 6 (5):610-1. doi: 10.1016/S15345807(04)00141-8

30. Ivanova S, Repnik U, Bojic L, Petelin A, Turk V, Turk B. Lysosomes in Apoptosis. Methods Enzymol (2008) 442:183-99. doi: 10.1016/S0076-6879(08)01409-2

31. Stavropoulou E, Pircalabioru GG, Bezirtzoglou E. The Role of Cytochromes P450 in Infection. Front Immunol (2018) 9:89. doi: 10.3389/fimmu.2018.00089

32. Benes P, Vetvicka V, Fusek M. Cathepsin D-many Functions of One Aspartic Protease. Crit Rev Oncol Hematol (2008) 68(1):12-28. doi: 10.1016/ j.critrevonc.2008.02.008

33. Masson O, Bach AS, Derocq D, Prébois C, Laurent-Matha V, Pattingre S, et al. Pathophysiological Functions of Cathepsin D: Targeting Its Catalytic Activity Versus Its Protein Binding Activity? Biochimie (2010) 92(11):1635-43. doi: 10.1016/j.biochi.2010.05.009

34. Pranjol MZ, Gutowski N, Hannemann M, Whatmore J. The Potential Role of the Proteases Cathepsin D and Cathepsin $L$ in the Progression and Metastasis of Epithelial Ovarian Cancer. Biomolecules (2015) 5(4):3260-79. doi: 10.3390/ biom5043260

35. Vetvicka V, Vetvickova J, Benes P. Role of Enzymatically Inactive Procathepsin D in Lung Cancer. Anticancer Res (2004) 24(5A):2739-43.

36. Zhao CF, Herrington DM. The Function of Cathepsins B, D, and X in Atherosclerosis. Am J Cardiovasc Dis (2016) 6(4):163-70.

37. Turk B. Targeting Proteases: Successes, Failures and Future Prospects. Nat Rev Drug Discov (2006) 5(9):785-99. doi: $10.1038 / \mathrm{nrd} 2092$

38. Ipsen DH, Lykkesfeldt J, Tveden-Nyborg P. Molecular Mechanisms of Hepatic Lipid Accumulation in Non-Alcoholic Fatty Liver Disease. Cell Mol Life Sci (2018) 75(18):3313-27. doi: 10.1007/s00018-018-2860-6

39. Thelen AM, Zoncu R. Emerging Roles for the Lysosome in Lipid Metabolism. Trends Cell Biol (2017) 27(11):833-50. doi: 10.1016/j.tcb.2017.07.006

40. Mizunoe Y, Sudo Y, Okita N, Hiraoka H, Mikami K, Narahara T, et al. Involvement of Lysosomal Dysfunction in Autophagosome Accumulation and Early Pathologies in Adipose Tissue of Obese Mice. Autophagy (2017) 13 (4):64253. doi: 10.1080/15548627.2016.1274850

41. Hendrikx T, Walenbergh SM, Hofker MH, Shiri-Sverdlov R. Lysosomal Cholesterol Accumulation: Driver on the Road to Inflammation During Atherosclerosis and non-Alcoholic Steatohepatitis. Obes Rev (2014) 15 (5):424-33. doi: 10.1111/obr.12159 
42. Mizunoe Y, Kobayashi M, Tagawa R, Nakagawa Y, Shimano H, Higami Y. Association Between Lysosomal Dysfunction and Obesity-Related Pathology: A Key Knowledge to Prevent Metabolic Syndrome. Int J Mol Sci (2019) 20(15). doi: 10.3390/ijms20153688

43. Miyazaki T, Matsuzaki Y. Taurine and Liver Diseases: A Focus on the Heterogeneous Protective Properties of Taurine. Amino Acids (2014) 46 (1):101-10. doi: 10.1007/s00726-012-1381-0

44. Dong S, Chen QL, Song YN, Sun Y, Wei B, Li XY, et al. Mechanisms of CCl4- Induced Liver Fibrosis With Combined Transcriptomic and Proteomic Analysis. J Toxicol Sci (2016) 41(4):561-72. doi: 10.2131/jts.41.561

45. Panebianco C, Oben JA, Vinciguerra M, Pazienza V. Senescence in Hepatic Stellate Cells as a Mechanism of Liver Fibrosis Reversal: A Putative Synergy Between Retinoic Acid and PPAR-Gamma Signalings. Clin Exp Med (2017) 17 (3):269-80. doi: 10.1007/s10238-016-0438-x

46. Schenten V, Plançon S, Jung N, Hann J, Bueb JL, Bréchard S, et al. Secretion of the Phosphorylated Form of S100A9 From Neutrophils Is Essential for the Proinflammatory Functions of Extracellular S100a8/A9. Front Immunol (2018) 9:447. doi: 10.3389/fimmu.2018.00447

47. Loser K, VogI T, Voskort M, Lueken A, Kupas V, Nacken W, et al. The Toll-Like Receptor 4 Ligands Mrp8 and Mrp14 Are Crucial in the Development of Autoreactive CD8+ T Cells. Nat Med (2010) 16(6):713-7. doi: 10.1038/nm.2150

48. Tang H, Pang S, Wang M, Xiao X, Rong Y, Wang H, et al. TLR4 Activation is Required for IL-17-Induced Multiple Tissue Inflammation and Wasting in Mice. J Immunol (2010) 185(4):2563-9. doi: 10.4049/jimmunol.0903664

49. Hou L, Cooley J, Swanson R, Ong PC, Pike RN, Bogyo M, et al. The Protease Cathepsin L Regulates Th17 Cell Differentiation. J Autoimmun (2015) 65:56-63. doi: 10.1016/j.jaut.2015.08.006

50. Singh RK, Haka AS, Asmal A, Barbosa-Lorenzi VC, Grosheva I, Chin HF, et al. TLR4 (Toll-Like Receptor 4)-Dependent Signaling Drives Extracellular Catabolism of LDL (Low-Density Lipoprotein) Aggregates. Arterioscler Thromb Vasc Biol (2020) 40(1):86-102. doi: 10.1161/ATVBAHA.119.313200

51. Hasan ST, Zingg JM, Kwan P, Noble T, Smith D, Meydani M. Curcumin Modulation of High Fat Diet-Induced Atherosclerosis and Steatohepatosis in LDL Receptor Deficient Mice. Atherosclerosis (2014) 232(1):40-51. doi: 10.1016/j.atherosclerosis.2013.10.016

52. Kaabia Z, Poirier J, Moughaizel M, Aguesse A, Billon-Crossouard S, Fall F, et al. Plasma Lipidomic Analysis Reveals Strong Similarities Between Lipid Fingerprints in Human, Hamster and Mouse Compared to Other Animal Species. Sci Rep (2018) 8(1):15893. doi: 10.1038/s41598-018-34329-3

53. Ishibashi S, Brown MS, Goldstein JL, Gerard RD, Hammer RE, Herz J. Hypercholesterolemia in Low Density Lipoprotein Receptor Knockout Mice and its Reversal by Adenovirus-Mediated Gene Delivery. J Clin Invest (1993) 92(2):883-93. doi: $10.1172 / \mathrm{JCl} 116663$

54. Yin W, Kuchibhotla SD, Guy E, Park YM, Nimako G, Vanegas D, et al. Plasma Lipid Profiling Across Species for the Identification of Optimal Animal Models of Human Dyslipidemia. J Lipid Res (2012) 53(1):51-65. doi: 10.1194/jlr.M019927

55. Kennedy DJ, Kuchibhotla SD, Guy E, Park YM, Nimako G, Vanegas D, et al. Dietary Cholesterol Plays a Role in CD36Mediated Atherogenesis in LDLR- Knockout Mice. Arterioscler Thromb Vasc Biol (2009) 29(10):1481-7. doi: 10.1161/ATVBAHA.109.191940

56. Wouters K, Shiri-Sverdlov R, van Gorp PJ, van Bilsen M, Hofker MH. Understanding Hyperlipidemia and Atherosclerosis: Lessons From Genetically Modified Apoe and Ldlr Mice. Clin Chem Lab Med (2005) 43 (5):470-9. doi: 10.1515/CCLM.2005.085

57. Bieghs V, Van Gorp PJ, Wouters K, Hendrikx T, Gijbels MJ, van Bilsen M, et al. LDL Receptor Knock-Out Mice Are a Physiological Model Particularly Vulnerable to Study the Onset of Inflammation in Non-Alcoholic Fatty Liver Disease. PLoS One (2012) 7(1):e30668. doi: 10.1371/journal.pone.0030668 


\section{Supplemental material}

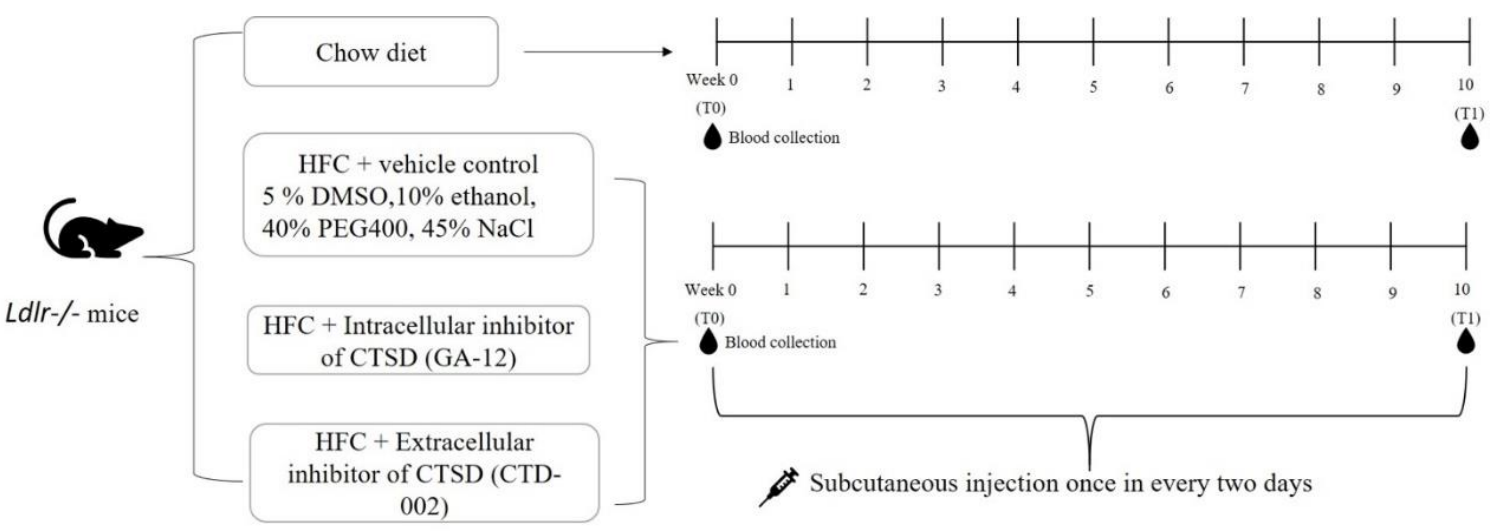

Figure S1 Schematic representation of the study design and the timeline of the in vivo experiment. Ldlr/- mice fed a regular chow diet were included as a control group for NASH disease phenotype $(n=20)$. HFC mice received vehicle or CTSD inhibitors subcutaneously once in every two days for 10 weeks. Sample sizes are $n=17$ for vehicle group, $n=20$ for intracellular inhibitor (GA-12)treated group and $n=17$ for extracellular inhibitor (CTD-002)-treated group. 
(A)

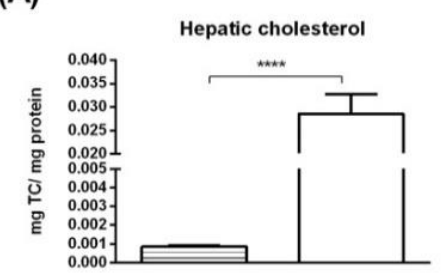

(C)

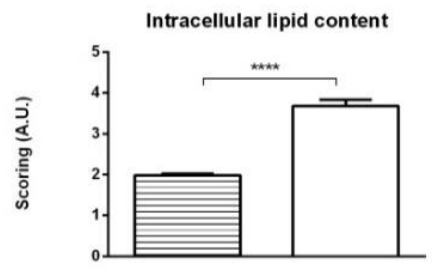

(D)

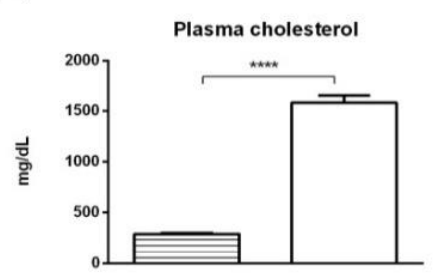

(F)

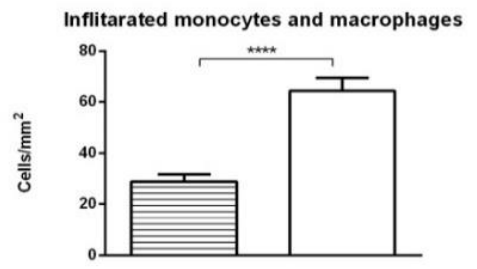

(G)

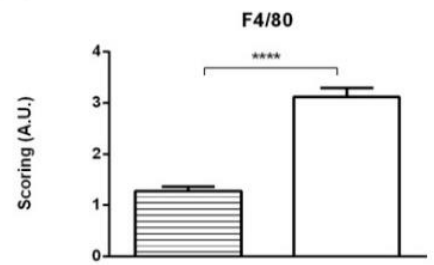

(B)

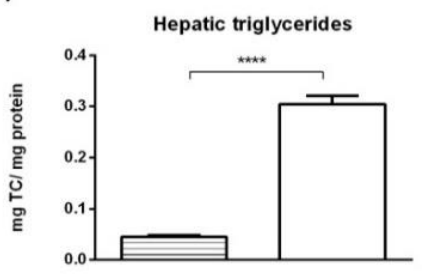

Chow

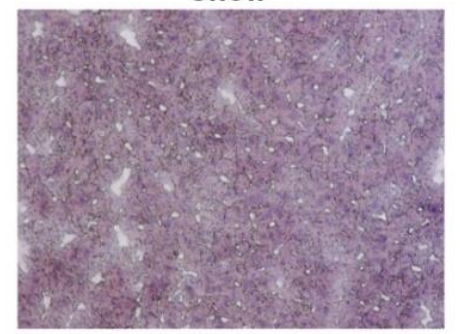

Plasma triglycerides

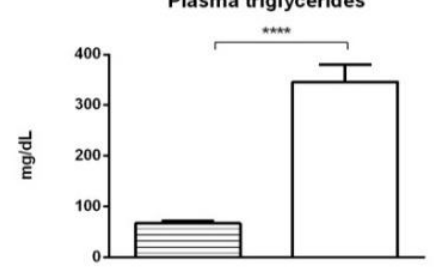

(E)
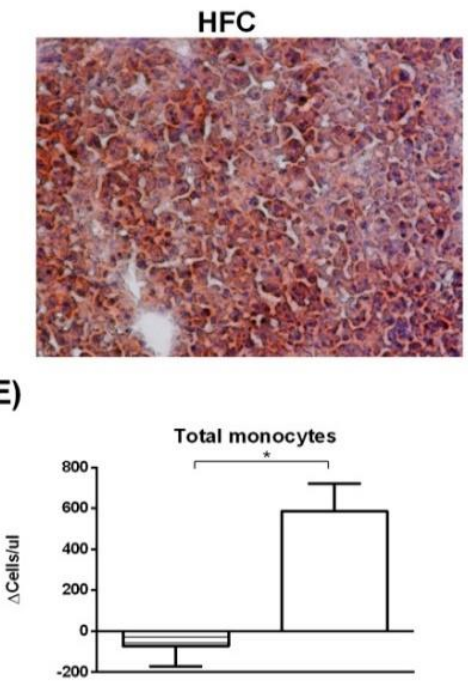

HFC
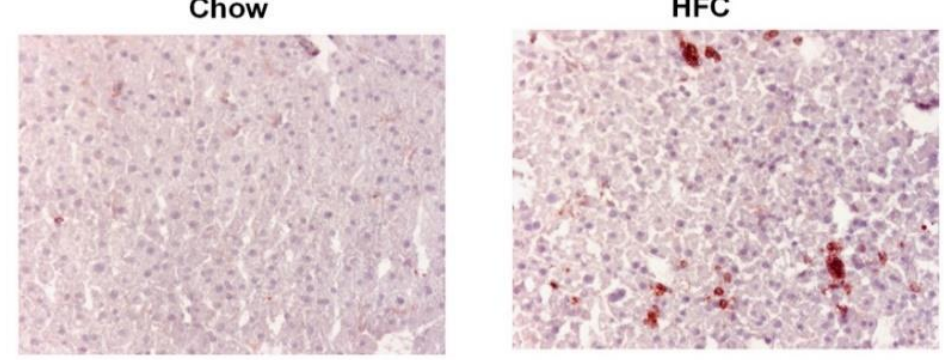

HFC

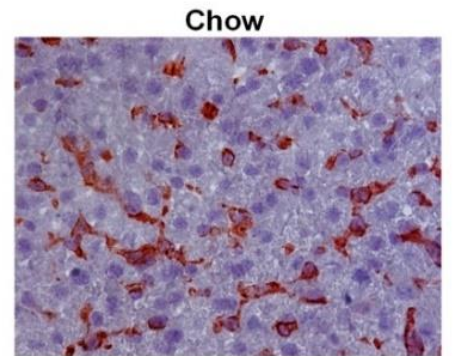

Chow control HFC Control

Figure S2 HFC supplementation for 10 week induces NASH in Ldlr-/- mice: (A-B) Feeding mice with HFC diet for 10 weeks lead to a significant increase in hepatic cholesterol and triglyceride levels compared to mice on chow diet. (C) Scoring of hepatic lipid content (oil red o staining) using arbitrary units (A.U.). (Original magnification, 200x) (D) Plasma lipid levels between chow and HFC mice. (E) Total number of plasma monocytes as measured by FACS. (F) Liver sections were stained for infiltrating macrophages and neutrophils (Mac-1). Positive cells were counted in six microscopical views (original magnification, 200x) (G) Hepatic immunostaining for F4/80. Postive cells were evaluated and scored according to arbitrary units (original magnification, 400x). Error bars represent \pm SEM; $n=17-20$ animals; $n=5$ per each group for FACS; * represents $p<0.05 * * * *$ represents $p<0.001$ compared to chow control mice as determined by two-tailed unpaired $t$ test. 
Chow

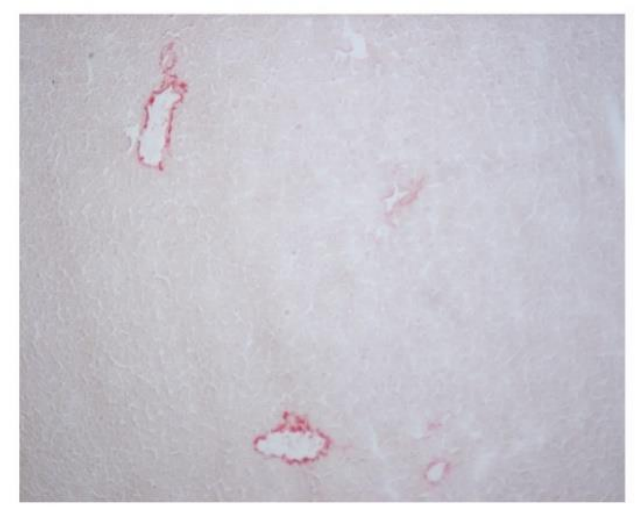

Intracellular CTSD inhibitor

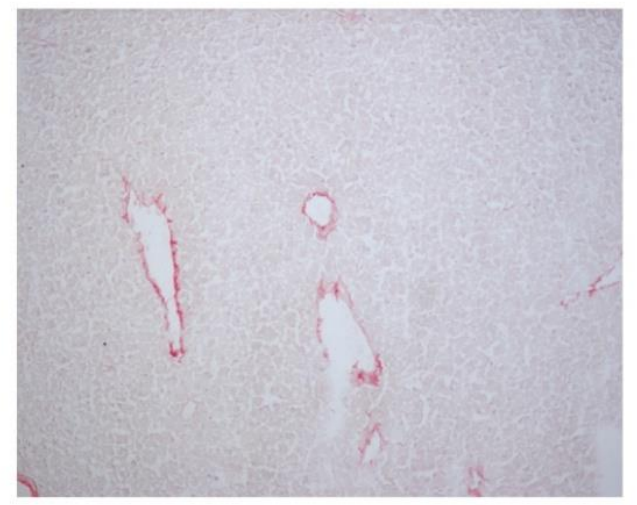

HFC

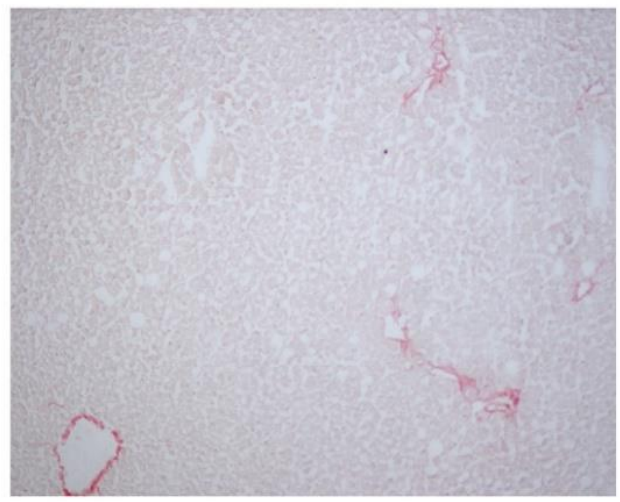

\section{Extracellular CTSD inhibitor}

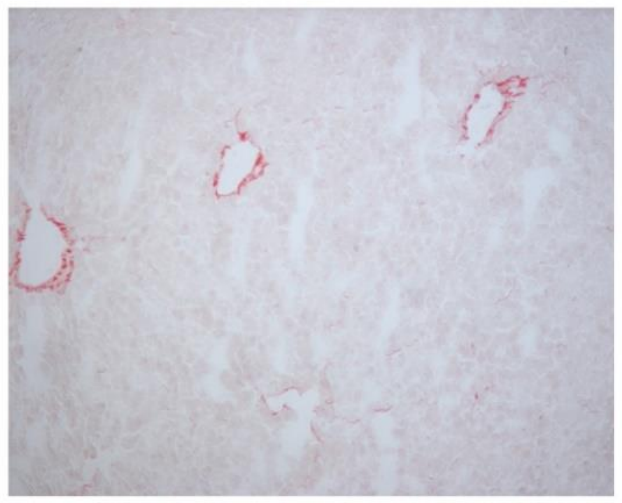

Figure S3 No changes in hepatic fibrosis between different experimental groups of mice: Representative pictures of Sirius Red staining (original magnification, 100x)

(A)
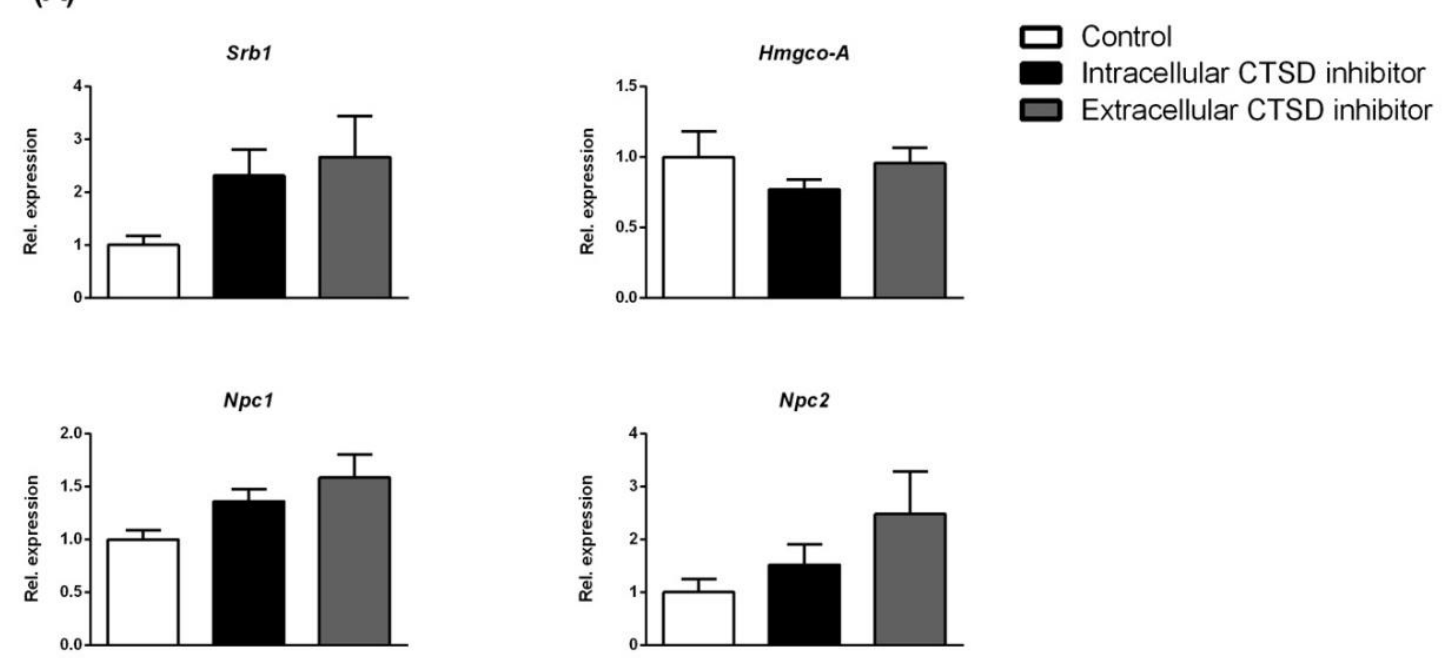

Figure S4. Hepatic gene expression of lipid-related genes: (A) Hepatic gene expression of lipid metabolismrelated genes. Error bars represent \pm SEM. $n=16-20$ per group. 
(A)

(B)

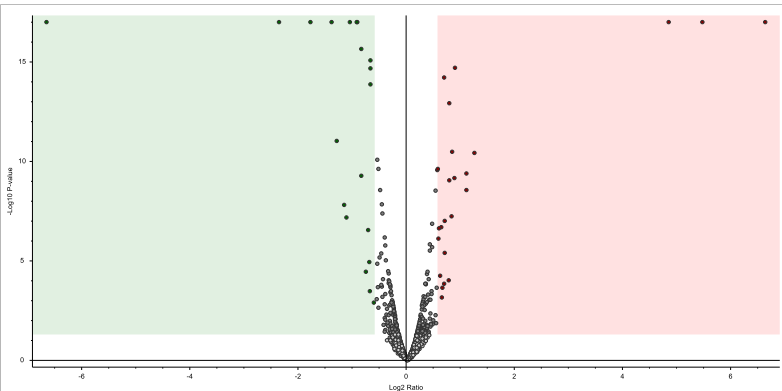

(C)
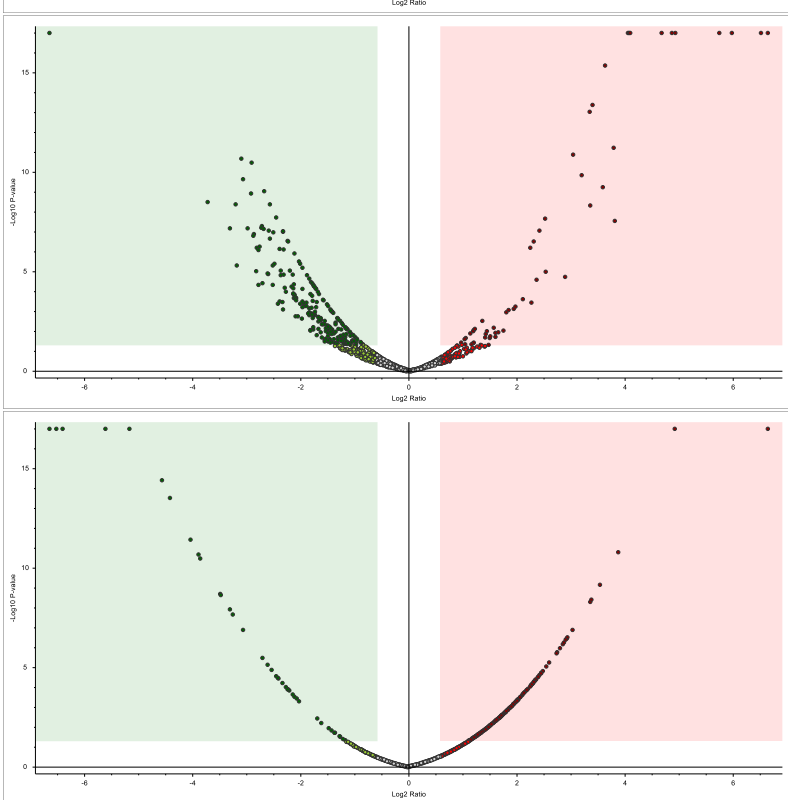

Figure S5. Label-free quantitative LC-MS proteomics of different groups of mice. Volcano plots of protein changes observed in the livers of mice belonging to different groups. The - $\log 10$ (BenjaminiHochberg corrected P value) is plotted against the log2 (fold change) for respective groups. (A) extracellular inhibitor-treated group vs control (B) intracellular inhibitor-treated group vs control and (C) extracellular vs intracellular inhibitor-treated groups.

Table S1: Significantly upregulated proteins found in control mice compared to intracellular inhibitor-treated mice. Proteins were selected with a ratio of at least 1.5 -fold change and $p$-value of $\leq 0.05$. In the table, Protein accession number according to UniProt, protein names, gene ID, abundance ratio $(\log 2 \geq 0.58)$ are listed.

\begin{tabular}{|c|c|c|c|}
\hline \multirow{2}{*}{$\begin{array}{l}\text { Accession } \\
\text { number }\end{array}$} & $\begin{array}{l}\text { Upregulated proteins in control mice compared to intracellular } \\
\text { inhibitor-treated mice }\end{array}$ & \multirow[t]{2}{*}{ Gene ID } & \multirow{2}{*}{$\begin{array}{c}\text { Control/ } \\
\text { intracellular } \\
\text { inhibitor } \\
\text { Abundance } \\
\text { ratio (log2) }\end{array}$} \\
\hline & Description & & \\
\hline Q9D6Y9 & 1,4-alpha-glucan-branching enzyme & Gbe1 & 1,26 \\
\hline Q9R092 & 17-beta-hydroxysteroid dehydrogenase type 6 & Hsd17b6 & 2,08 \\
\hline P61922 & 4-aminobutyrate aminotransferase, mitochondrial & Abat & 1,4 \\
\hline A0JNU3 & 60 kDa lysophospholipase & Aspg & 1,46 \\
\hline Q9DCD0 & 6-phosphogluconate dehydrogenase, decarboxylating & Pgd & 1,78 \\
\hline Q80XL6 & Acyl-CoA dehydrogenase family member 11 & Acad11 & 1,35 \\
\hline Q99PL7 & Acyl-CoA desaturase & Scd3 & 3,31 \\
\hline Q5XG73 & Acyl-CoA-binding domain-containing protein 5 & Acbd5 & 2,6 \\
\hline P51881 & ADP/ATP translocase 2 & Slc25a5 & 1,75 \\
\hline P47740 & Aldehyde dehydrogenase family 3 member A2 & Aldh3a2 & 1,79 \\
\hline Q8BW75 & Amine oxidase [flavin-containing] B & Maob & 1,49 \\
\hline Q91YI0 & Arginosuccinate lyase & Asl & 1,8 \\
\hline P34927 & Asialoglycoprotein receptor 1 & Asgr1 & 1,79 \\
\hline Q8BP47 & Asparagine--tRNA ligase, cytoplasmic & NARS1 & 1,79 \\
\hline P04919 & Band 3 anion transport protein & Slc4a1 & 2,13 \\
\hline
\end{tabular}




\begin{tabular}{|c|c|c|c|}
\hline P18572 & Basigin & Bsg & 1,61 \\
\hline Q61335 & B-cell receptor-associated protein 31 & Bcap31 & 1,83 \\
\hline Q9QXX4 & Calcium-binding mitochondrial carrier protein Aralar2 & Slc25a13 & 2,03 \\
\hline Q61490 & CD166 antigen & Alcam & 1,77 \\
\hline Q91WS0 & CDGSH iron-sulfur domain-containing protein 1 & Cisd1 & 2,14 \\
\hline P60766 & Cell division control protein 42 homolog & Cdc42 & 2,37 \\
\hline Q68FD5 & Clathrin heavy chain 1 & Cltc & 1,43 \\
\hline Q9QZE5 & Coatomer subunit gamma-1 & Copg1 & 1,77 \\
\hline Q9QZQ8 & Core histone macro-H2A.1 & H2afy & 1,89 \\
\hline P50172 & Corticosteroid 11-beta-dehydrogenase isozyme 1 & Hsd11b1 & 1,88 \\
\hline Q99L04 & Dehydrogenase/reductase SDR family member 1 & Dhrs1 & 2,59 \\
\hline P50285 & Dimethylaniline monooxygenase [ $\mathrm{N}$-oxide-forming] 1 & Fmo1 & 2,72 \\
\hline P97501 & Dimethylaniline monooxygenase [ $\mathrm{N}$-oxide-forming] 3 & Fmo3 & 2,38 \\
\hline P97872 & Dimethylaniline monooxygenase [N-oxide-forming] 5 & Fmo5 & 2,57 \\
\hline 054734 & $\begin{array}{l}\text { Dolichyl-diphosphooligosaccharide--protein glycosyltransferase } 48 \text { kDa } \\
\text { subunit }\end{array}$ & Ddost & 1,67 \\
\hline Q91YQ5 & $\begin{array}{l}\text { Dolichyl-diphosphooligosaccharide--protein glycosyltransferase } \\
\text { subunit } 1\end{array}$ & Rpn1 & 2,31 \\
\hline P58252 & Elongation factor 2 & Eef2 & 1,66 \\
\hline Q8BHI7 & Elongation of very long chain fatty acids protein 5 & Elovl5 & 3,2 \\
\hline P19096 & $\begin{array}{l}\text { Fatty acid synthase } \\
\end{array}$ & Fasn & 2,24 \\
\hline 008914 & Fatty-acid amide hydrolase 1 & Faah & 1,55 \\
\hline P35576 & Glucose-6-phosphatase & G6pc & 2,12 \\
\hline Q64516 & Glycerol kinase & Gk & 1,73 \\
\hline Q9ET01 & Glycogen phosphorylase, liver form & Pygl & 1,51 \\
\hline P01901 & $\mathrm{H}-2$ class I histocompatibility antigen, $\mathrm{K}-\mathrm{B}$ alpha chain & $\mathrm{H} 2-\mathrm{K} 1$ & 1,58 \\
\hline P10922 & Histone $\mathrm{H} 1$. & $\mathrm{H} 1-0$ & 2,72 \\
\hline P15864 & Histone H1.2 & $\mathrm{H} 1-2$ & 2,48 \\
\hline $\mathrm{P} 43274$ & Histone H1.4 & $\mathrm{H} 1-4$ & 2,91 \\
\hline P43276 & Histone $\mathrm{H} 1.5$ & $\mathrm{H} 1-5$ & 2,61 \\
\hline P02301 & Histone $\mathrm{H} 3.3 \mathrm{C}$ & $\mathrm{H} 3 \mathrm{f3c}$ & 1,57 \\
\hline P62806 & Histone $\mathrm{H} 4$ & $\mathrm{H} 4 \mathrm{c} 1$ & 1,3 \\
\hline Q8VCZ9 & Hydroxyproline dehydrogenase & Prodh2 & 2,82 \\
\hline P01872 & Immunoglobulin heavy constant mu & Ighm & 1,43 \\
\hline P70168 & Importin subunit beta-1 & Kpnb1 & 1,97 \\
\hline Q9CXF0 & $\begin{array}{l}\text { Kynureninase } \\
\end{array}$ & Kynu & 1,67 \\
\hline Q9WVM8 & Kynurenine/alpha-aminoadipate aminotransferase, mitochondrial & Aadat & 2,16 \\
\hline Q922Q8 & Leucine-rich repeat-containing protein 59 & Lrrc59 & 2,76 \\
\hline P58710 & L-gulonolactone oxidase & Gulo & 2,12 \\
\hline P41216 & Long-chain-fatty-acid--CoA ligase 1 & Acsl1 & 1,68 \\
\hline Q8JZR0 & Long-chain-fatty-acid--CoA ligase 5 & Acsl5 & 1,84 \\
\hline 035114 & Lysosome membrane protein 2 & Scarb2 & 2,1 \\
\hline Q99J39 & Malonyl-CoA decarboxylase, mitochondrial & Mlycd & 1,62 \\
\hline 055022 & Membrane-associated progesterone receptor component 1 & Pgrmc1 & 1,58 \\
\hline Q80UU9 & Membrane-associated progesterone receptor component 2 & Pgrmc2 & 2,33 \\
\hline Q9DD20 & Methyltransferase-like protein 7B & Mettl7b & 2,33 \\
\hline Q91VS7 & Microsomal glutathione S-transferase 1 & Mgst1 & 2,91 \\
\hline Q922Q1 & Mitochondrial amidoxime reducing component 2 & Marc2 & 2 \\
\hline Q9CW42 & Mitochondrial amidoxime-reducing component 1 & Marc1 & 1,79 \\
\hline Q791V5 & Mitochondrial carrier homolog 2 & Mtch2 & 2,86 \\
\hline Q9CZW5 & Mitochondrial import receptor subunit TOM70 & Tomm70 & 1,63 \\
\hline Q9D023 & $\begin{array}{l}\text { Mitochondrial pyruvate carrier } 2 \text { OS=Mus musculus OX=10090 } \\
\qquad \mathrm{GN}=\mathrm{Mpc} 2 \mathrm{PE}=1 \mathrm{SV}=1\end{array}$ & Mpc2 & 1,93 \\
\hline Q8VDD5 & Myosin-9 OS=Mus musculus & Myh9 & 1,71 \\
\hline Q9ERS2 & NADH dehydrogenase [ubiquinone] 1 alpha subcomplex subunit 13 & Ndufa13 & 1,86 \\
\hline 009111 & $\begin{array}{l}\text { NADH dehydrogenase [ubiquinone] } 1 \text { beta subcomplex subunit 11, } \\
\text { mitochondrial }\end{array}$ & Ndufb11 & 2,39 \\
\hline Q9CQC7 & NADH dehydrogenase [ubiquinone] 1 beta subcomplex subunit 4 & Ndufb4 & 1,73 \\
\hline Q9DC70 & $\begin{array}{l}\text { NADH dehydrogenase [ubiquinone] iron-sulfur protein 7, } \\
\text { mitochondrial }\end{array}$ & Ndufs7 & 2 \\
\hline Q9DCN2 & NADH-cytochrome b5 reductase 3 & Cyb5r3 & 2,45 \\
\hline Q9QXD1 & Peroxisomal acyl-coenzyme A oxidase 2 & Acox2 & 1,84 \\
\hline P97372 & Proteasome activator complex subunit 2 & Psme2 & 2,13 \\
\hline Q9CQS8 & $\begin{array}{l}\text { Protein transport protein Sec61 subunit beta OS=Mus musculus } \\
\qquad \mathrm{OX}=10090 \mathrm{GN}=\mathrm{Sec} 61 \mathrm{~b} \mathrm{PE}=1 \mathrm{SV}=3\end{array}$ & Sec61b & 3,18 \\
\hline P35293 & Ras-related protein Rab-18 & Rab18 & 1,67 \\
\hline P35278 & Ras-related protein Rab-5C & Rab5c & 2,09 \\
\hline
\end{tabular}


Extracellular cathepsin D in NASH

\begin{tabular}{|c|c|c|c|}
\hline P51150 & Ras-related protein Rab-7a & Rab7a & 2,51 \\
\hline Q9JM62 & Receptor expression-enhancing protein 6 & Reep6 & 1,49 \\
\hline 088451 & Retinol dehydrogenase 7 & Rdh7 & 2,23 \\
\hline Q9D5J6 & Sedoheptulokinase & Shpk & 1,89 \\
\hline P52430 & Serum paraoxonase/arylesterase 1 & Pon1 & 1,45 \\
\hline Q9JJL3 & Solute carrier organic anion transporter family member 1B2 & Slco1b2 & 3,72 \\
\hline Q62465 & Synaptic vesicle membrane protein VAT-1 homolog & Vat1 & 2,13 \\
\hline Q9D0R2 & Threonine--tRNA ligase 1, cytoplasmic & Tars1 & 2,12 \\
\hline Q9QUI0 & Transforming protein RhoA & Rhoa & 2,27 \\
\hline Q62186 & Translocon-associated protein subunit delta & Ssr4 & 1,97 \\
\hline Q99KF1 & Transmembrane emp24 domain-containing protein 9 & Tmed9 & 2,41 \\
\hline Q8JZU2 & Tricarboxylate transport protein, mitochondrial & Slc25a1 & 2,52 \\
\hline P68373 & Tubulin alpha-1C chain & Tuba1c & 1,17 \\
\hline P68372 & Tubulin beta-4B chain & Tubb4b & 1,21 \\
\hline Q8BWQ1 & UDP-glucuronosyltransferase $2 \mathrm{~A} 3$ & Ugt2a3 & 2,81 \\
\hline P17717 & UDP-glucuronosyltransferase 2B17 & Ugt2b17 & 2,29 \\
\hline Q8JZZO & UDP-glucuronosyltransferase 3A2 & Ugt3a2 & 2,78 \\
\hline 035488 & Very long-chain acyl-CoA synthetase & Slc27a2 & 2,32 \\
\hline 070503 & Very-long-chain 3-oxoacyl-CoA reductase & Hsd17b12 & 1,79 \\
\hline Q9QY76 & Vesicle-associated membrane protein-associated protein B & Vapb & 2,09 \\
\hline 008547 & Vesicle-trafficking protein SEC22b & Sec22b & 2,11 \\
\hline P25444 & 40S ribosomal protein S2 & Rps2 & 1,32 \\
\hline P97461 & 40S ribosomal protein S5 & Rps5 & 2,98 \\
\hline Q6ZWV3 & $60 S$ ribosomal protein $\mathrm{L} 10$ & Rpl10 & 1,24 \\
\hline P19253 & $60 S$ ribosomal protein $\mathrm{L} 13 \mathrm{a}$ & Rpl13a & 1,5 \\
\hline Q9CR57 & $60 S$ ribosomal protein L14 & Rpl14 & 1,7 \\
\hline Q9CZM2 & $60 S$ ribosomal protein $\mathrm{L} 15$ & Rpl15 & 1,75 \\
\hline P35980 & $60 S$ ribosomal protein $\mathrm{L} 18$ & Rpl18 & 2,69 \\
\hline Q8BP67 & $60 S$ ribosomal protein $\mathrm{L} 24$ & Rpl24 & 1,71 \\
\hline P41105 & $60 S$ ribosomal protein $\mathrm{L} 28$ & Rpl28 & 1,74 \\
\hline P47915 & $60 S$ ribosomal protein $\mathrm{L} 29$ & Rpl29 & 1,55 \\
\hline Q9D1R9 & $60 S$ ribosomal protein $\mathrm{L} 34$ & Rpl34 & 2,36 \\
\hline Q6ZWV7 & $60 S$ ribosomal protein L35 & Rpl35 & 2,08 \\
\hline P83882 & $60 S$ ribosomal protein $\mathrm{L} 36 \mathrm{a}$ & Rpl36a & 1,64 \\
\hline Q9D8E6 & $60 S$ ribosomal protein L4 & Rpl4 & 1,73 \\
\hline P47911 & $60 S$ ribosomal protein L6 & Rpl6 & 2,91 \\
\hline P14148 & $60 \mathrm{~S}$ ribosomal protein $\mathrm{L7}$ & Rpl7 & 3,09 \\
\hline P12970 & $60 S$ ribosomal protein $\mathrm{L7a}$ & Rpl7a & 1,31 \\
\hline Q9CQX2 & Cytochrome b5 type B & Cyb5b & 1,84 \\
\hline P00405 & Cytochrome c oxidase subunit 2 & Mtco2 & 2,51 \\
\hline P19783 & Cytochrome c oxidase subunit 4 isoform 1 , mitochondrial & Cox4i1 & 1,96 \\
\hline Q9CPQ1 & Cytochrome c oxidase subunit 6C & Cox6c & 2,19 \\
\hline P20852 & Cytochrome P450 2A5 & Сур2a5 & 2,78 \\
\hline Q64458 & Cytochrome P450 2C29 & Cyp2c29 & 1,35 \\
\hline P56654 & Cytochrome P450 2C37 & Cyp2c37 & 2,32 \\
\hline P56657 & Cytochrome P450 2 C40 & Cyp2c40 & 1,94 \\
\hline P24456 & Cytochrome P450 2D10 & Cyp2d10 & 1,82 \\
\hline Q8CIM7 & Cytochrome P450 2D26 & Cyp2d26 & 3,06 \\
\hline Q05421 & Cytochrome P450 2E1 & Cyp2e1 & 2,68 \\
\hline Q64459 & Cytochrome P450 3A11 & Cyp3a11 & 2,87 \\
\hline Q9JMA7 & Cytochrome P450 3A41 & Сур3а41а & 1,77 \\
\hline Q9CQQ7 & ATP synthase $F(0)$ complex subunit B1, mitochondrial & Atp5pb & 1,41 \\
\hline P03930 & ATP synthase protein 8 & Mtatp8 & 1,97 \\
\hline P56379 & ATP synthase subunit ATP5MPL, mitochondrial & Atp5mpl & 2,71 \\
\hline Q9CPQ8 & ATP synthase subunit g, mitochondrial & Atp5mg & 1,63 \\
\hline P55096 & ATP-binding cassette sub-family D member 3 & Abcd3 & 2,57 \\
\hline
\end{tabular}


Table S2: Significantly upregulated proteins found in control mice compared to extracellular inhibitor-treated mice. Proteins were selected with a ratio of at least 1.5 -fold change and $p$-value of $\leq \mathbf{0 . 0 5}$. In the table, Protein accession number according to UniProt, protein names, gene ID, abundance ratio $(\log 2 \geq 0.58)$ are listed.

\begin{tabular}{|c|c|c|c|}
\hline \multirow{2}{*}{$\begin{array}{l}\text { Accession } \\
\text { number }\end{array}$} & $\begin{array}{l}\text { Upregulated proteins in response in control mice compared to } \\
\text { extracellular inhibitor-treated mice }\end{array}$ & \multirow[t]{2}{*}{ Gene Id } & \multirow{2}{*}{$\begin{array}{c}\begin{array}{c}\text { Control/ } \\
\text { extracellular } \\
\text { inhibitor }\end{array} \\
\text { Abundance } \\
\text { ratio (log2) }\end{array}$} \\
\hline & Description & & \\
\hline Ca1 & Carbonic anhydrase 1 & Ca1 & 0,9 \\
\hline Col14a1 & Collagen alpha-1(XIV) chain & Col14a1 & 0,82 \\
\hline $\mathrm{Ckm}$ & Creatine kinase M-type & $\mathrm{Ckm}$ & 0,65 \\
\hline Fabp3 & Fatty acid-binding protein, heart & Fabp3 & 0,69 \\
\hline Gsta1 & Glutathione S-transferase A1 & Gsta1 & 0,66 \\
\hline $\mathrm{Hbb}-\mathrm{b} 1$ & Hemoglobin subunit beta-1 & $\mathrm{Hbb}-\mathrm{b} 1$ & 0,65 \\
\hline Ighv3-6 & Ig heavy chain $V$ region 3-6 & Ighv3-6 & 1,28 \\
\hline Nubpl & Iron-sulfur protein NUBPL & Nubpl & 0,59 \\
\hline $\mathrm{Mb}$ & Myoglobin & $\mathrm{Mb}$ & 2,35 \\
\hline Myl1 & Myosin light chain $1 / 3$, skeletal muscle isoform & Myl1 & 1,04 \\
\hline Prelp & Prolargin & Prelp & 0,74 \\
\hline S100a8 & Protein S100-A8 & S100a8 & 0,83 \\
\hline S100a9 & Protein S100-A9 & S100a9 & 0,65 \\
\hline Spta1 & Spectrin alpha chain, erythrocytic 1 & Spta1 & 0,68 \\
\hline Tnnc2 & Troponin C, skeletal muscle & Tnnc2 & 1,37 \\
\hline Xylt2 & Xylosyltransferase 2 & Xylt2 & 0,91 \\
\hline
\end{tabular}

Table S3: Significantly upregulated proteins found in extracellular inhibitor-treated mice compared to control mice. Proteins were selected with a ratio of at least 1.5 -fold change and $p$-value of $\leq \mathbf{0 . 0 5}$. In the table, Protein accession number according to UniProt, protein names, gene ID, abundance ratio $(\log 2 \geq 0.58)$ are listed.

\begin{tabular}{|c|c|c|c|}
\hline \multirow[t]{2}{*}{$\begin{array}{l}\text { Accession } \\
\text { number }\end{array}$} & $\begin{array}{l}\text { Upregulated proteins in extracellular inhibitor-treated mice compared } \\
\text { to control mice }\end{array}$ & \multirow[t]{2}{*}{ Gene Id } & \multirow{2}{*}{$\begin{array}{c}\text { Extracellular } \\
\text { inhibitor/ } \\
\text { control }\end{array}$} \\
\hline & Description & & \\
\hline Q99PL7 & Acyl-CoA desaturase 3 & Scd3 & 0,6 \\
\hline P07361 & Alpha-1-acid glycoprotein 2 & Orm2 & 1,12 \\
\hline Q9DBE0 & Cysteine sulfinic acid decarboxylase & Csad & 0,8 \\
\hline Q05816 & Fatty acid-binding protein 5 & Fabp5 & 0,71 \\
\hline P01864 & Ig gamma-2A chain $\mathrm{C}$ region secreted form & & 0,91 \\
\hline P01655 & Ig kappa chain V-III region PC 7132 & & 0,8 \\
\hline Q8K2C9 & Very-long-chain (3R)-3-hydroxyacyl-CoA dehydratase 3 & Hacd3 & 0,86 \\
\hline Q9R1Z8 & Vinexin & Sorbs3 & 0,68 \\
\hline P18242 & Cathepsin D & Ctsd & 0,58 \\
\hline 035403 & Amine sulfotransferase & Sult3a1 & 0,64 \\
\hline P00186 & Cytochrome P450 1A2 & Cyp1a2 & 0,59 \\
\hline 054749 & Cytochrome P450 2J5 & Cyp2j5 & 0,71 \\
\hline Q64481 & Cytochrome P450 3A16 & Cyp3a16 & 1,12 \\
\hline P04938 & Major urinary protein 11 & Mup11 & 0,66 \\
\hline P11589 & Major urinary protein 2 & Mup2 & 0,61 \\
\hline P09528 & Ferritin heavy chain & Fth1 & 0,85 \\
\hline P29391 & Ferritin light chain 1 & Ftl1 & 0,9 \\
\hline P46978 & $\begin{array}{c}\text { Dolichyl-diphosphooligosaccharide--protein glycosyltransferase } \\
\text { subunit STT3A }\end{array}$ & Stt3a & 0,72 \\
\hline Q99KR7 & Peptidyl-prolyl cis-trans isomerase $\mathrm{F}$, mitochondrial & Ppif & 0,71 \\
\hline P54726 & UV excision repair protein RAD23 homolog A & $\operatorname{Rad} 23 a$ & 0,66 \\
\hline
\end{tabular}


Table S4: Significantly upregulated proteins found in extracellular inhibitor-treated mice compared to intracellular inhibitor-treated mice. Proteins were selected with a ratio of at least 1.5-fold change and $p$ value of $\leq \mathbf{0 . 0 5}$. In the table, Protein accession number according to UniProt, protein names, gene ID, abundance ratio $(\log 2 \geq 0.58)$ are listed.

\begin{tabular}{|c|c|c|c|}
\hline \multirow[t]{2}{*}{$\begin{array}{l}\text { Accession } \\
\text { number }\end{array}$} & $\begin{array}{l}\text { Upregulated proteins in extracellular inhibitor-treated mice } \\
\text { compared to Intracellular inhibitor-treated mice }\end{array}$ & \multirow[t]{2}{*}{ Gene ID } & \multirow{2}{*}{$\begin{array}{c}\text { Extracellular } \\
\text { inhibitor/ } \\
\text { intracellular } \\
\text { inhibitor } \\
\text { Abundance } \\
\text { ratio (log2) }\end{array}$} \\
\hline & Description & & \\
\hline P19096 & Fatty acid synthase & Fasn & 1,91 \\
\hline Q9ET01 & Glycogen phosphorylase, liver form & Pygl & 1,66 \\
\hline Q9DCN2 & NADH-cytochrome b5 reductase 3 & Cyb5r3 & 2,19 \\
\hline Q791V5 & Mitochondrial carrier homolog 2 & Mtch2 & 2,42 \\
\hline P35576 & Glucose-6-phosphatase & G6pc & 3,54 \\
\hline Q91YI0 & Argininosuccinate lyase & Asl & 1,61 \\
\hline P51881 & ADP/ATP translocase 2 & Slc25a5 & 1,66 \\
\hline P41216 & Long-chain-fatty-acid--CoA ligase 1 & Acsl1 & 1,71 \\
\hline Q9CZM2 & 60S ribosomal protein L15 & Rpl15 & 1,61 \\
\hline $\mathrm{P} 47740$ & Aldehyde dehydrogenase family 3 member A2 & Aldh3a2 & 1,73 \\
\hline P50172 & Corticosteroid 11-beta-dehydrogenase isozyme 1 & Hsd11b1 & 1,74 \\
\hline Q9DD20 & Methyltransferase-like protein 7B & Mettl7b & 2,42 \\
\hline P19783 & Cytochrome c oxidase subunit 4 isoform 1 , mitochondrial & Cox4i1 & 1,79 \\
\hline P01872 & Immunoglobulin heavy constant mu & Ighm & 1,72 \\
\hline P58710 & L-gulonolactone oxidase & Gulo & 1,73 \\
\hline Q922Q1 & Mitochondrial amidoxime reducing component 2 & Marc2 & 2 \\
\hline 035488 & Very long-chain acyl-CoA synthetase & Slc27a2 & 1,7 \\
\hline Q8JZRO & Long-chain-fatty-acid--CoA ligase 5 & Acsl5 & 1,65 \\
\hline Q9QXX4 & Calcium-binding mitochondrial carrier protein Aralar2 & Slc25a13 & 2,03 \\
\hline P43274 & Histone $\mathrm{H} 1.4$ & $\mathrm{H} 1-4$ & 1,74 \\
\hline 088451 & Retinol dehydrogenase 7 & Rdh7 & 1,79 \\
\hline Q91WS0 & CDGSH iron-sulfur domain-containing protein 1 & Cisd1 & 1,83 \\
\hline P00405 & Cytochrome c oxidase subunit 2 & Mtco2 & 2,35 \\
\hline P97872 & Dimethylaniline monooxygenase [ $\mathrm{N}$-oxide-forming] 5 & Fmo5 & 2,29 \\
\hline Q9CPQ1 & Cytochrome c oxidase subunit 6C & Cox6c & 2,35 \\
\hline Q64516 & Glycerol kinase OS=Mus musculus OX=10090 GN=Gk PE=1 SV=2 & Gk & 1,78 \\
\hline Q9DCD0 & 6-phosphogluconate dehydrogenase, decarboxylating & Pgd & 1,95 \\
\hline Q9DCM2 & Glutathione S-transferase kappa 1 & Gstk1 & 1,7 \\
\hline Q91YQ5 & $\begin{array}{l}\text { Dolichyl-diphosphooligosaccharide--protein glycosyltransferase } \\
\text { subunit } 1\end{array}$ & Rpn1 & 1,86 \\
\hline Q9CW42 & Mitochondrial amidoxime-reducing component 1 & Marc1 & 1,82 \\
\hline Q9CXF0 & Kynureninase & Kynu & 1,73 \\
\hline P03930 & ATP synthase protein 8 & Mtatp8 & 1,9 \\
\hline Q8BP67 & $60 S$ ribosomal protein $\mathrm{L} 24$ & Rpl24 & 1,58 \\
\hline P35980 & 60S ribosomal protein $\mathrm{L} 18$ & Rpl18 & 2,06 \\
\hline Q9D0R2 & Threonine--tRNA ligase 1, cytoplasmic & Tars1 & 2,1 \\
\hline Q8CIM7 & Cytochrome P450 2D26 & Cyp2d26 & 2,6 \\
\hline Q64458 & Cytochrome P450 2C29 & Cyp2c29 & 1,85 \\
\hline Q99L04 & Dehydrogenase/reductase SDR family member 1 & Dhrs1 & 2,17 \\
\hline P14148 & 60S ribosomal protein $\mathrm{L7}$ & Rpl7 & 2,73 \\
\hline P55096 & ATP-binding cassette sub-family D member 3 & Abcd3 & 2,27 \\
\hline Q9QXD1 & Peroxisomal acyl-coenzyme A oxidase 2 & Acox2 & 2,03 \\
\hline P47911 & $60 S$ ribosomal protein L6 & Rpl6 & 1,86 \\
\hline Q9D5J6 & Sedoheptulokinase & Shpk & 1,91 \\
\hline P97372 & Proteasome activator complex subunit 2 & Psme2 & 1,69 \\
\hline Q9QUI0 & Transforming protein RhoA & Rhoa & 2,34 \\
\hline P50285 & Dimethylaniline monooxygenase [ $\mathrm{N}$-oxide-forming] 1 & Fmo1 & 2,07 \\
\hline Q91X44 & Glucokinase regulatory protein & Gckr & 1,67 \\
\hline P24456 & Cytochrome P450 2D10 & Cyp2d10 & 2,15 \\
\hline Q9D1R9 & $60 S$ ribosomal protein $\mathrm{L} 34$ & Rpl34 & 2,3 \\
\hline P34927 & Asialoglycoprotein receptor 1 & Asgr1 & 1,61 \\
\hline Q922Q8 & Leucine-rich repeat-containing protein 59 & Lrrc59 & 2,25 \\
\hline Q9CZW5 & Mitochondrial import receptor subunit TOM70 & Tomm70 & 1,59 \\
\hline P97461 & 40S ribosomal protein S5 & Rps5 & 2,85 \\
\hline P15864 & Histone $\mathrm{H} 1.2$ & $\mathrm{H} 1-2$ & 1,69 \\
\hline
\end{tabular}




\begin{tabular}{|c|c|c|c|}
\hline P10922 & Histone $\mathrm{H} 1.0$ & $\mathrm{H} 1-0$ & 1,83 \\
\hline P17717 & UDP-glucuronosyltransferase 2B17 & Ugt2b17 & 2,28 \\
\hline Q9CQC7 & NADH dehydrogenase [ubiquinone] 1 beta subcomplex subunit 4 & Ndufb4 & 1,67 \\
\hline Q61335 & B-cell receptor-associated protein 31 & Bcap31 & 1,58 \\
\hline Q99J39 & Malonyl-CoA decarboxylase, mitochondrial & Mlycd & 1,58 \\
\hline Q9WVM8 & Kynurenine/alpha-aminoadipate aminotransferase, mitochondrial & Aadat & 2,02 \\
\hline P60766 & Cell division control protein 42 homolog & Cdc42 & 2,38 \\
\hline Q8BWQ1 & UDP-glucuronosyltransferase $2 \mathrm{~A} 3$ & Ugt2a3 & 2,75 \\
\hline Q62465 & Synaptic vesicle membrane protein VAT-1 homolog & Vat1 & 1,93 \\
\hline P56654 & Cytochrome P450 2C37 & Cyp2c37 & 1,78 \\
\hline P97501 & Dimethylaniline monooxygenase [ $\mathrm{N}$-oxide-forming] 3 & Fmo3 & 1,98 \\
\hline P56135 & ATP synthase subunit $f$, mitochondrial & Atp5mf & 1,95 \\
\hline Q9CQX2 & Cytochrome b5 type B & Cyb5b & 2,01 \\
\hline 035114 & Lysosome membrane protein 2 & Scarb2 & 2,12 \\
\hline Q8R1I1 & Cytochrome b-c1 complex subunit 9 & Uqcr10 & 1,77 \\
\hline A2AKK5 & Acyl-coenzyme A amino acid N-acyltransferase 1 & Acnat1 & 1,59 \\
\hline Q9DC70 & $\begin{array}{l}\text { NADH dehydrogenase [ubiquinone] iron-sulfur protein 7, } \\
\text { mitochondrial }\end{array}$ & Ndufs7 & 1,95 \\
\hline 008547 & Vesicle-trafficking protein SEC22b & $\operatorname{Sec} 22 b$ & 1,73 \\
\hline P61027 & Ras-related protein Rab-10 & Rab10 & 1,56 \\
\hline Q62186 & Translocon-associated protein subunit delta & Ssr4 & 1,58 \\
\hline P56657 & Cytochrome P450 2C40 & Cyp2c40 & 1,98 \\
\hline Q9QY76 & Vesicle-associated membrane protein-associated protein B & Vapb & 1,79 \\
\hline P18572 & Basigin & Bsg & 1,82 \\
\hline Q64514 & Tripeptidyl-peptidase 2 & Tpp2 & 1,71 \\
\hline Q8JZZO & UDP-glucuronosyltransferase 3A2 & Ugt3a2 & 1,88 \\
\hline Q9D023 & Mitochondrial pyruvate carrier 2 & Mpc2 & 1,76 \\
\hline Q8JZU2 & Tricarboxylate transport protein, mitochondrial & Slc25a1 & 2,32 \\
\hline P00186 & Cytochrome P450 1A2 & Cyp1a2 & 2,39 \\
\hline Q8BP47 & Asparagine--tRNA ligase, cytoplasmic & NARS1 & 1,72 \\
\hline Q64459 & Cytochrome P450 3A11 & Cyp3a11 & 2 \\
\hline Q5XG73 & Acyl-CoA-binding domain-containing protein 5 & Acbd5 & 2,45 \\
\hline B1AR13 & CDGSH iron-sulfur domain-containing protein 3, mitochondrial & Cisd3 & 1,85 \\
\hline 070503 & Very-long-chain 3-oxoacyl-CoA reductase & Hsd17b12 & 1,64 \\
\hline Q9JMA7 & Cytochrome P450 3A41 & Сур3а41a & 2,87 \\
\hline 009111 & $\begin{array}{l}\text { NADH dehydrogenase [ubiquinone] } 1 \text { beta subcomplex subunit 11, } \\
\text { mitochondrial }\end{array}$ & Ndufb11 & 2,48 \\
\hline Q8BHI7 & Elongation of very long chain fatty acids protein 5 & Elovl5 & 2,55 \\
\hline Q8VCZ9 & Hydroxyproline dehydrogenase & Prodh2 & 2,91 \\
\hline P51150 & Ras-related protein Rab-7a & Rab7a & 2,26 \\
\hline P04919 & Band 3 anion transport protein & Slc4a1 & 1,67 \\
\hline P35278 & Ras-related protein Rab-5C & Rab5c & 1,92 \\
\hline Q9CR61 & NADH dehydrogenase [ubiquinone] 1 beta subcomplex subunit 7 & Ndufb7 & 1,81 \\
\hline Q9R092 & 17-beta-hydroxysteroid dehydrogenase type 6 & Hsd17b6 & 1,82 \\
\hline P56379 & ATP synthase subunit ATP5MPL, mitochondrial & Atp5mpl & 3,36 \\
\hline Q925I1 & ATPase family AAA domain-containing protein 3 & Atad3 & 1,63 \\
\hline Q80UU9 & Membrane-associated progesterone receptor component 2 & Pgrmc2 & 2,13 \\
\hline Q99PL7 & Acyl-CoA desaturase & Scd3 & 2,8 \\
\hline Q9CQS8 & Protein transport protein Sec61 subunit beta & Sec61b & 3,03 \\
\hline Q9R1J0 & Sterol-4-alpha-carboxylate 3-dehydrogenase, decarboxylating & Nsdhl & 1,78 \\
\hline Q99KF1 & Transmembrane emp24 domain-containing protein 9 & Tmed9 & 2,27 \\
\hline Q9R1P3 & Proteasome subunit beta type- 2 & Psmb2 & 1,72 \\
\hline P15392 & Cytochrome P450 2A4 & Cyp2a4 & 2,21 \\
\hline Q9CRC0 & Vitamin K epoxide reductase complex subunit 1 & Vkorc1 & 1,88 \\
\hline 009117 & Synaptophysin-like protein 1 & Sypl1 & 1,79 \\
\hline P20852 & Cytochrome P450 2A5 & Cyp2a5 & 2,92 \\
\hline P43276 & Histone $\mathrm{H} 1.5$ & $\mathrm{H} 1-5$ & 1,57 \\
\hline Q9CQW9 & Interferon-induced transmembrane protein 3 & Ifitm3 & 1,79 \\
\hline Q9D2R0 & Acetoacetyl-CoA synthetase & Aacs & 2,02 \\
\hline Q9CQC6 & Basic leucine zipper and W2 domain-containing protein 1 & Bzw1 & 1,68 \\
\hline Q9JJL3 & Solute carrier organic anion transporter family member 1B2 & Slco1b2 & 3,87 \\
\hline Q64669 & NAD(P)H dehydrogenase [quinone] & Nqo1 & 1,83 \\
\hline Q76M72 & Solute carrier family 22 member 27 & Slc22a27 & 2,31 \\
\hline Q61490 & CD166 antigen & Alcam & 1,65 \\
\hline Q05421 & Cytochrome P450 2E1 & Cyp2e1 & 2,07 \\
\hline P20852 & Cytochrome P450 2A5 & Cyp2a5 & 2,92 \\
\hline P15392 & Cytochrome P450 2A4 & Сур2a4 & 2,21 \\
\hline
\end{tabular}




\begin{tabular}{cccc}
\hline Q9JMA7 & Cytochrome P450 3A41 & Cyp3a41a & 2,87 \\
\hline Q64459 & Cytochrome P450 3A11 & Cyp3a11 & 2 \\
\hline P00186 & Cytochrome P450 1A2 & Cyp1a2 & 2,39 \\
\hline P56657 & Cytochrome P450 2C40 & Cyp2c40 & 1,98 \\
\hline Q9CQX2 & Cytochrome b5 type B & Cyb5b & 2,01 \\
\hline P56654 & Cytochrome P450 2C37 & Cyp2c37 & 1,78 \\
\hline Q64458 & Cytochrome P450 2C29 & Cyp2c29 & 1,85 \\
\hline Q8CIM7 & Cytochrome P450 2D26 & Cyp2d26 & 2,6 \\
\hline Q9DCN2 & NADH-cytochrome b5 reductase 3 & Cyb5r3 & 2,19 \\
\hline
\end{tabular}

Table S5: Significantly upregulated proteins found in intracellular inhibitor-treated mice compared to control mice. Proteins were selected with a ratio of at least 1.5 -fold change and $p$-value of $\leq 0.05$. In the table, Protein accession number according to UniProt, protein names, gene ID, abundance ratio $(\log 2 \geq 0.58)$ are listed.

\begin{tabular}{|c|c|c|c|}
\hline \multirow{2}{*}{$\begin{array}{l}\text { Accession } \\
\text { number }\end{array}$} & $\begin{array}{l}\text { Upregulated proteins in intracellular inhibitor-treated mice } \\
\text { compared to control mice }\end{array}$ & \multirow[t]{2}{*}{ Gene ID } & \multirow{2}{*}{$\begin{array}{c}\begin{array}{c}\text { Intracellular } \\
\text { inhibitor/ } \\
\text { control }\end{array} \\
\text { Abundance } \\
\text { ratio (log2) }\end{array}$} \\
\hline & Description & & \\
\hline Q9R0Y5 & Adenylate kinase isoenzyme 1 & Ak1 & 4,1 \\
\hline P21550 & Beta-enolase & Eno3 & 5,97 \\
\hline 054724 & Caveolae-associated protein 1 & Cavin1 & 2,37 \\
\hline P07310 & Creatine kinase M-type & $\mathrm{Ckm}$ & 4,05 \\
\hline Q6P8J7 & Creatine kinase S-type, mitochondrial & Ckmt2 & 3,2 \\
\hline P31001 & Desmin & Des & 2,42 \\
\hline P11404 & Fatty acid-binding protein, heart & Fabp3 & 3,04 \\
\hline P97447 & Four and a half LIM domains protein 1 & Fhl1 & 3,4 \\
\hline P14602 & Heat shock protein beta-1 & Hspb1 & 1,98 \\
\hline Q5EBG6 & Heat shock protein beta- 6 & Hspb6 & 3,79 \\
\hline Q9JKS4 & LIM domain-binding protein 3 & Ldb3 & 5,75 \\
\hline P16125 & L-lactate dehydrogenase B chain & Ldhb & 2,11 \\
\hline P04247 & Myoglobin OS=Mus musculus & $\mathrm{Mb}$ & 6,52 \\
\hline Q62234 & Myomesin-1 OS=Mus musculus & Myom1 & 3,59 \\
\hline P05977 & Myosin light chain $1 / 3$, skeletal muscle isoform & Myl1 & 3,64 \\
\hline P51667 & Myosin regulatory light chain 2 , ventricular/cardiac muscle isoform & Myl2 & 4,68 \\
\hline Q5SX40 & Myosin-1 OS=Mus musculus & Myh1 & 2,31 \\
\hline Q5SX39 & Myosin-4 OS=Mus musculus & Myh4 & 1,81 \\
\hline Q91Z83 & Myosin-7 OS=Mus musculus & Myh7 & 1,85 \\
\hline Q9JK37 & Myozenin-1 & Myoz1 & 2,54 \\
\hline Q9JJW5 & Myozenin-2 & Myoz2 & 1,95 \\
\hline P00688 & Pancreatic alpha-amylase & Amy2 & 2,89 \\
\hline 070250 & Phosphoglycerate mutase 2 & Pgam2 & 4,87 \\
\hline Q9R1C7 & Pre-mRNA-processing factor 40 homolog $\mathrm{A}$ & Prpf40a & 2,27 \\
\hline P58771 & Tropomyosin alpha-1 chain & Tpm1 & 3,35 \\
\hline P58774 & Tropomyosin beta chain & Tpm2 & 4,93 \\
\hline P20801 & Troponin C, skeletal muscle & Tnnc2 & 4,08 \\
\hline P13412 & Troponin I, fast skeletal muscle & Tnni2 & 2,25 \\
\hline Q9QZ47 & Troponin T, fast skeletal muscle & Tnnt3 & 2,53 \\
\hline Q11136 & Xaa-Pro dipeptidase & Pepd & 3,81 \\
\hline
\end{tabular}


Table S6: Significantly upregulated proteins found in intracellular inhibitor-treated mice compared to extracellular inhibitor-treated mice. Proteins were selected with a ratio of at least 1.5 -fold change and $p$ value of $\leq \mathbf{0 . 0 5}$. In the table, Protein accession number according to UniProt, protein names, gene ID, abundance ratio $(\log 2 \geq 0.58)$ are listed.

\begin{tabular}{|c|c|c|c|}
\hline \multirow[t]{2}{*}{$\begin{array}{l}\text { Accession } \\
\text { number }\end{array}$} & $\begin{array}{l}\text { Upregulated proteins in intracellular inhibitor-treated mice } \\
\text { compared to extracellular inhibitor-treated mice }\end{array}$ & \multirow[t]{2}{*}{ Gene ID } & \multirow{2}{*}{$\begin{array}{c}\text { Intracellular } \\
\text { inhibitor/ } \\
\text { extracellular } \\
\text { inhibitor } \\
\text { Abundance } \\
\text { ratio (log2) }\end{array}$} \\
\hline & Description & & \\
\hline Q5SX40 & Myosin-1 & Myh1 & 2,54 \\
\hline P07310 & Creatine kinase M-type & $\mathrm{Ckm}$ & 5,61 \\
\hline P58771 & Tropomyosin alpha-1 chain & Tpm1 & 3,31 \\
\hline P05977 & Myosin light chain $1 / 3$, skeletal muscle isoform & Myl1 & 3,07 \\
\hline P58774 & Tropomyosin beta chain & Tpm2 & 5,16 \\
\hline Q9QZ47 & Troponin T, fast skeletal muscle & Tnnt3 & 3,48 \\
\hline P21550 & Beta-enolase & Eno3 & 4,56 \\
\hline P31001 & Desmin & Des & 2,71 \\
\hline P13412 & Troponin I, fast skeletal muscle & Tnni2 & 2,42 \\
\hline P97447 & Four and a half LIM domains protein 1 & Fhl1 & 3,47 \\
\hline P11404 & Fatty acid-binding protein, heart & Fabp3 & 6,52 \\
\hline Q9R0Y5 & Adenylate kinase isoenzyme 1 & Ak1 & 3,89 \\
\hline 070250 & Phosphoglycerate mutase 2 & Pgam2 & 4,04 \\
\hline Q62234 & Myomesin-1 & Myom1 & 3,85 \\
\hline Q5SX39 & Myosin-4 & Myh4 & 2,08 \\
\hline P16125 & L-lactate dehydrogenase B chain & Ldhb & 2,03 \\
\hline Q9JK37 & Myozenin-1 & Myoz1 & 2,45 \\
\hline P14602 & Heat shock protein beta-1 & Hspb1 & 2,14 \\
\hline 054724 & Caveolae-associated protein 1 & Cavin1 & 2,33 \\
\hline Q9JJW5 & Myozenin-2 & Myoz2 & 2,11 \\
\hline Q91WK1 & SPRY domain-containing protein 4 & Spryd4 & 1,62 \\
\hline P14602 & Heat shock protein beta-1 & Hspb1 & 2,14 \\
\hline
\end{tabular}

Table S7: Top 10 enriched pathways upon intracellular CTSD inhibition

(A) KEGG database (Mouse) - Intracellular inhibitor-treated mice/ control

\begin{tabular}{ccc}
\hline Name & P-value (p) & Combined Score \\
\hline Hypertrophic cardiomyopathy (HCM) & $1.711 \mathrm{e}-7$ & 603.92 \\
\hline Dilated cardiomyopathy (DCM) & $2.150 \mathrm{e}-7$ & 568.61 \\
\hline Cardiac muscle contraction & 0.000005430 & 414.48 \\
\hline Glycolysis / Gluconeogenesis & 0.0001367 & 265.60 \\
\hline Adrenergic signaling in cardiomyocytes & 0.00006793 & 172.92 \\
\hline Thiamine metabolism & 0.02227 & 169.08 \\
\hline Arginine and proline metabolism & 0.002548 & 159.27 \\
\hline Tight junction & 0.001967 & 74.63 \\
\hline Propanoate metabolism & 0.04550 & 66.45 \\
\hline Glucagon signaling pathway & 0.01021 & 59.93 \\
\hline
\end{tabular}


(B) KEGG database (Mouse) -control/ intracellular inhibitor-treated mice

\begin{tabular}{ccc}
\hline Name & P-value (p) & Combined Score \\
\hline Steroid hormone biosynthesis & $6.332 \mathrm{e}-13$ & 573.81 \\
\hline Ribosome & $1.294 \mathrm{e}-15$ & 519.41 \\
\hline Fatty acid biosynthesis & 0.00009699 & 300.03 \\
\hline Retinol metabolism & $8.955 \mathrm{e}-9$ & 277.69 \\
\hline Chemical carcinogenesis & $1.195 \mathrm{e}-8$ & 264.64 \\
\hline Linoleic acid metabolism & $9.232 \mathrm{e}-7$ & 252.64 \\
\hline Ascorbate and aldarate metabolism & 0.0007320 & 121.54 \\
\hline Peroxisome & 0.00001966 & 117.28 \\
\hline PPAR signaling pathway & 0.00002104 & 115.18 \\
\hline Drug metabolism & 0.00001064 & 106.53 \\
\hline
\end{tabular}





\section{Chapter 5}

\section{Inflammatory Bowel Disease: A stressed \\ "gut/feeling"}

Yvonne Oligschlaeger, Tulasi Yadati, Tom Houben, Claudia Maria Condello Oliván, Ronit Shiri-Sverdlov 


\title{
Chapter 5
}

\begin{abstract}
Inflammatory bowel disease (IBD) is a chronic and relapsing intestinal inflammatory condition, hallmarked by a disturbance in the bidirectional interaction between gut and brain. In general, the gut/brain axis involves direct and/or indirect communication via the central and enteric nervous system, host innate immune system, and particularly the gut microbiota. This complex interaction implies that IBD is a complex multifactorial disease. There is increasing evidence that stress adversely affects the gut/microbiota/brain axis by altering intestinal mucosa permeability and cytokine secretion, thereby influencing the relapse risk and disease severity of IBD. Given the recurrent nature, therapeutic strategies particularly aim at achieving and maintaining remission of the disease. Alternatively, these strategies focus on preventing permanent bowel damage and concomitant longterm complications. In this review, we discuss the gut/microbiota/brain interplay with respect to chronic inflammation of the gastrointestinal tract and particularly shed light on the role of stress. Hence, we evaluated the therapeutic impact of stress management in IBD.
\end{abstract}




\section{Introduction}

Inflammatory bowel disease (IBD) is a chronic and relapsing disorder [1], including Crohn's disease and ulcerative colitis. While Crohn's disease is characterized by transmural inflammation in any part of the gastrointestinal tract, ulcerative colitis is affecting the mucosal layer of the colon and rectum. Similar to other immune-mediated chronic diseases, such as rheumatoid arthritis, IBD is hallmarked by periods of remission interspersed with periods of acute flare. During disease course, symptoms like abdominal pain, cramping, loose stools or bloody diarrhea, fatigue, anemia and/or weight loss can manifest. The prevalence and incidence of IBD are increasing enormously [2]. Together with its early onset, relapsing nature, and life-threatening complications, IBD is currently a major health issue. Although the exact pathogenesis is unclear, IBD is certainly driven by disturbed crosstalk between a variety of parameters, i.e., genetic susceptibility and internal and external factors [3], which will be discussed in more detail. Although treatment options mainly focus on reducing intestinal inflammation [1], achieving/maintaining remission or improving the patient's quality-oflife, no cure for IBD is currently available. Given that IBD is a systemic disease, often associated with comorbidities such as anxiety and depression, this narrative review aims to evaluate the mutual interplay between stress and the gut/microbiota/brain axis, particularly with regard to chronic inflammation of the gastrointestinal tract. These insights set the basis for better understanding the impact of stress management on disease activity in IBD.

\section{Search strategy}

For this narrative review, we selected peer-reviewed preclinical and clinical articles as well as metaanalyses and important reviews from the PubMed database between January 1980 and June 2019. The following search terms were used: inflammatory bowel disease/IBD, Crohn's disease, ulcerative colitis, irritable bowel syndrome, gut/intestines, microbiota/microbiome, brain, interplay/axis, psychological/chronic/acute/cognitive stress, lifestyle factors, stress management, stress resilience, inflammation, disease activity, therapies/interventions/therapeutic strategies.

\section{Gut/Microbiota/Brain interplay}

In this section, we will focus on the tight association between the brain and gut, discussing the involvement of endocrine, immune, and neural pathways as well as the gut microbiota.

\subsection{Brain/Gut interaction}

The hypothalamic pituitary adrenal (HPA) axis is an endocrine pathway belonging to the limbic system of the brain. In response to stress [4], the activated HPA axis causes the secretion of corticotropinreleasing factor (CRF) from the hypothalamus, which stimulates the pituitary gland to release adrenocorticotropic hormone (ACTH). In turn, ACTH triggers the immunosuppressive stress-hormone cortisol from the adrenal cortex [5], which ordinarily induces the synthesis of anti-inflammatory cytokines. However, in response to stress, sustained cortisol activity has also been associated with pro-inflammatory responses [6]. Likewise, stress-induced cortisol was shown to increase intestinal 
barrier dysfunction, as recently shown by crypt analyses from rodents and humans [7]. Moreover, administration of cortisol in a porcine model caused a shift in microbiota composition, [8], pointing towards a role for cortisol in regulating intestinal inflammation and altering microbiota composition.

In addition to the HPA axis, the autonomic nervous system (ANS) coordinates the function of the gastrointestinal tract. The ANS is known to trigger efferent signals from the central nervous system (CNS; i.e., brain and spinal cord) to the intestinal wall to regulate mucosal immune responses [9] and other intestinal functions, such as nutrient absorption [10]. Vice versa, via enteric, spinal, and vagal nerves, afferent signals from the intestinal lumen are also known to regulate behavior, sleep, and stress reactivity $[11,12]$. Upon receiving stimuli from the diet and gut microbiota $[13,14]$, the enteric nervous system (ENS, "second brain"), which is part of the peripheral nervous system, mainly communicates with the CNS in a bidirectional manner. However, the ENS is also capable of intrinsically innervating the gut [15] in an autonomous manner [16].

\subsection{Gut microbiota}

The gastrointestinal tract serves as a dynamic and local ecosystem for gut microbiota. Whereas, often being classified into two major phyla, i.e., Bacteroidetes and Firmicutes [17], the gut microbiota is composed of over 35,000 bacterial species [18]. Besides playing a role in metabolism [19], it is also essential for controlling processes related to barrier function against pathogenic microorganism colonization, such as mucosal integrity [20], immunomodulation [21], and pathogen protection [22]. Recently, preclinical [23], translational [24], and clinical [25] studies suggested that alterations in the structural composition or function of the microbiome can contribute to the development of mental illness, including depression-like behavior, and thus, is a vital component linking the gut/brain axis. In line, data have indicated strong correlations between alterations in gut microbiota and the development of multifactorial chronic inflammatory disorders, such as IBD [26,27], suggesting that dysbiosis is an important factor in both gastrointestinal and mental health.

Intestinal bacteria and their metabolites are also involved in gut-associated neuroimmune mechanisms that influence mood and behavior leading to depression. These mechanisms include tryptophan metabolism as well as neural signaling within the ENS [28]. Tryptophan is an essential amino acid, derived from the diet. While crossing the blood-brain barrier and acting as a precursor of the neurotransmitter serotonin, tryptophan can also be degraded in the gut through the kynurenine and serotonin synthesis pathways. This degradation can affect its availability to pass the blood-brain barrier. Thus, by modulating tryptophan levels, microbiota can affect the brain, resulting in behavioral changes [29].

By fermentation of dietary fibers, the gut microbiota is also responsible for producing short-chain fatty acids (SCFAs), including butyric acid, propionic acid, and acetic acid, which are typically found to be reduced in mucosa and feces of patients with IBD [30]. As extensively reviewed by Parada Venegas et al. [31], these metabolic products have shown to play an important role in promoting epithelial cell proliferation [32], barrier function [33], and cellular metabolism [34]. In addition, SCFAs have been involved in controlling intestinal inflammation through activation of G-protein coupled receptor signaling pathways [35], thereby regulating intestinal homeostasis and inhibiting pathogen colonization. Relevantly, SCFAs are also known to exert neuroprotective properties. For instance, gamma-aminobutyric acid is an inhibitory neurotransmitter involved in anxiety and depression and 
can therefore modulate behavior [36]. Other mechanisms by which the intestinal microbiota affect neural responses include alterations in bacterial neurometabolites or bacterial cell wall sugars. These products can either act directly on primary afferent axons or trigger epithelial cells to release molecules that modulate neural signaling within the ENS [28].

Altogether, the multifaceted interplay between the gut, microbiota, and brain allows for intestinal and extraintestinal homeostasis, thereby coordinating gastrointestinal functions and modulating mood and higher cognitive functions, respectively.

\section{Gut/Microbiota/Brain interplay in IBD development}

It has become evident that factors such as genetics, environment, diet, and lifestyle are involved in dysregulation of the gut/microbiota/brain interplay, which in this section, will be discussed in the context of IBD development (Figure 1 and Table 1) [37,38].

Table 1: Factors involved in the gut/microbiota/brain interplay in inflammatory bowel disease (IBD) development

\begin{tabular}{|c|c|c|c|c|c|c|}
\hline Factor & Type of study & $\mathbf{N}$ & $\begin{array}{l}\text { Intervention/ } \\
\text { Methodology }\end{array}$ & Outcome & Author(s) & Reference \\
\hline \multirow{8}{*}{ Genetics } & Clinical study & 637 & Questionnaire & $\begin{array}{l}\text { First-degree relatives } \\
\text { have } 10 \text {-fold increased } \\
\text { risk of IBD development }\end{array}$ & Orholm, M. et al. & 37 \\
\hline & Meta-analysis & $\begin{array}{l}>75,000 \\
\text { cases \& } \\
\text { controls }\end{array}$ & GWAS & $\begin{array}{l}\text { Identified } 30 \text { gene loci } \\
\text { for } C D \text { and } 23 \text { for UC }\end{array}$ & Jostins, L. et al. & 39 \\
\hline & $\begin{array}{c}\text { Trans-ancestry } \\
\text { association } \\
\text { studies }\end{array}$ & 238,401 & GWAS & $\begin{array}{c}\text { Identified } 38 \\
\text { susceptibility loci for IBD }\end{array}$ & Liu, J.Z. et al. & 40 \\
\hline & $\begin{array}{c}\text { Genetic } \\
\text { association study }\end{array}$ & 6228 & Association studies & $\begin{array}{l}\text { Identified UPS and CYLD } \\
\text { gene are important in } \\
\text { IBD pathogenesis }\end{array}$ & Cleynen, I. et al. & 41 \\
\hline & $\begin{array}{c}\text { Genotype } \\
\text { association study }\end{array}$ & 34,819 & Association studies & $\begin{array}{l}\text { Insights into genetic } \\
\text { heterogeneity between } \\
\text { ileal and colonic CD }\end{array}$ & Cleynen, I. et al. & 42 \\
\hline & Clinical study & 323 & $\begin{array}{c}\text { Array-based } \\
\text { transcriptome data }\end{array}$ & $\begin{array}{l}\text { Identified } 99 \text { strong } \\
\text { positional candidate } \\
\text { genes in } 63 \text { risk loci }\end{array}$ & $\begin{array}{c}\text { Momozawa, Y. et } \\
\text { al. }\end{array}$ & 43 \\
\hline & Clinical study & $\begin{array}{l}189 \text { twin } \\
\text { pairs }\end{array}$ & Questionnaire & $\begin{array}{l}\text { Results highlight the } \\
\text { importance of } \\
\text { environmental triggers }\end{array}$ & $\begin{array}{l}\text { Spehlmann, ME. } \\
\text { et al. }\end{array}$ & 45 \\
\hline & Clinical study & $\begin{array}{l}80 \text { twin } \\
\text { pairs }\end{array}$ & Questionnaire & $\begin{array}{c}\text { Genetic influence is } \\
\text { stronger in } C D \text { than in } \\
\text { UC }\end{array}$ & $\begin{array}{l}\text { Halfvarson, J. et } \\
\text { al. }\end{array}$ & 46 \\
\hline \multirow{5}{*}{ Diet } & $\begin{array}{l}\text { Interventional, } \\
\text { open-label, } \\
\text { pilot study }\end{array}$ & 16 & $\begin{array}{c}\text { Vitamin D3 } \\
\text { supplementation }\end{array}$ & $\begin{array}{l}\text { Vitamin D3 modulates } \\
\text { the gut microbiome }\end{array}$ & Bashir, M. et al. & 48 \\
\hline & $\begin{array}{l}\text { In-vivo mouse } \\
\text { study }\end{array}$ & $\begin{array}{l}\text { 4-8 mice } \\
\text { per group }\end{array}$ & Oral antibiotics & $\begin{array}{c}\text { Increased levels of } \\
\text { intracellular zinc led to } \\
\text { bacterial clearance }\end{array}$ & Lahiri, A. et al. & 49 \\
\hline & $\begin{array}{l}\text { In-vivo mouse } \\
\text { study }\end{array}$ & $\begin{array}{l}\text { 4-5 mice } \\
\text { per group }\end{array}$ & $\begin{array}{l}\text { High-fat diet and oral } \\
\text { antibiotics }\end{array}$ & $\begin{array}{l}\text { High-fat diet alters gut } \\
\text { microbiome } \\
\text { composition }\end{array}$ & Hildebrandt, MA. & 55 \\
\hline & $\begin{array}{l}\text { In-vivo mouse } \\
\text { study }\end{array}$ & $\begin{array}{l}>100 \\
\text { inbred } \\
\text { mouse } \\
\text { strains }\end{array}$ & $\begin{array}{l}\text { High-fat/high-sucrose } \\
\text { diet }\end{array}$ & $\begin{array}{l}\text { High-fat/high-sucrose } \\
\text { diet influences gut } \\
\text { microbiota composition }\end{array}$ & Parks, BW. et al. & 56 \\
\hline & $\begin{array}{l}\text { Case-control } \\
\text { study }\end{array}$ & 86 & Dietary intake & $\begin{array}{c}\text { Mono- and } \\
\text { polyunsaturated fats } \\
\text { consumption is a risk } \\
\text { factor for IBD }\end{array}$ & Geerling, BJ. et al. & 57 \\
\hline
\end{tabular}




\begin{tabular}{|c|c|c|c|c|c|c|}
\hline & $\begin{array}{l}\text { In-vivo mouse } \\
\text { study }\end{array}$ & $\begin{array}{l}\text { 4-5 mice } \\
\text { per group }\end{array}$ & $\begin{array}{c}\text { Diet enriched with } \\
\text { phytosterols }\end{array}$ & $\begin{array}{c}\text { Phytosterols are } \\
\text { protective against IBD }\end{array}$ & Aldini, R. et al. & 58 \\
\hline \multirow{2}{*}{ Environment } & $\begin{array}{l}\text { Population-based } \\
\text { case-control study }\end{array}$ & 1382 & $\begin{array}{l}\text { Questionnaire on } 25 \\
\text { different topics }\end{array}$ & $\begin{array}{c}\text { Altered intestinal } \\
\text { microbiota may } \\
\text { modulate risk of IBD }\end{array}$ & Ng, SC. et al. & 62 \\
\hline & $\begin{array}{l}\text { Retrospective } \\
\text { case study }\end{array}$ & 1194 & $\begin{array}{l}\text { Clinical history and } \\
\text { questionnaire }\end{array}$ & $\begin{array}{c}\text { Higher prevalence of CD } \\
\text { in urban areas and UC in } \\
\text { inland areas }\end{array}$ & Carpio, D. et al. & 68 \\
\hline \multirow{2}{*}{ Smoking } & Meta-analysis & 245 articles & Smoking & $\begin{array}{c}\text { Smoking is a risk factor } \\
\text { for IBD }\end{array}$ & Mahid, SS. et al. & 51 \\
\hline & $\begin{array}{l}\text { Prospective case- } \\
\text { control study }\end{array}$ & 160 & $\begin{array}{c}\text { Transdermal nicotine } \\
\text { or placebo patches }\end{array}$ & $\begin{array}{l}\text { Smoking effects gut } \\
\text { microbiota composition }\end{array}$ & $\begin{array}{c}\text { Richardson, CE. et } \\
\text { al. }\end{array}$ & 52 \\
\hline \multirow{3}{*}{ Medication } & Meta-analysis & $\begin{array}{c}11 \\
\text { observatio } \\
\text { nal studies } \\
\end{array}$ & Antibiotic exposure & $\begin{array}{c}\text { Antibiotics increases the } \\
\text { risk of new-onset CD } \\
\text { than UC }\end{array}$ & Ungaro, R. et al. & 70 \\
\hline & Meta-analysis & 20 studies & $\mathrm{OCP}$ & $\begin{array}{c}\text { Increased risk for } \\
\text { development of } C D \text { and } \\
\text { UC }\end{array}$ & Ortizo, R. et al. & 71 \\
\hline & $\begin{array}{l}\text { Case-control } \\
\text { study }\end{array}$ & 122 & NSAIDs & $\begin{array}{c}\text { Provoked disease } \\
\text { activity in IBD }\end{array}$ & Felder, JB. et al. & 72 \\
\hline \multirow{3}{*}{ Exercise } & $\begin{array}{l}\text { In-vivo mouse } \\
\text { study }\end{array}$ & $\begin{array}{l}4 \text { mice per } \\
\text { group }\end{array}$ & Exercise training & $\begin{array}{c}\text { Alleviated symptoms of } \\
\text { acute colitis }\end{array}$ & Saxena, A. et al. & 60 \\
\hline & $\begin{array}{l}\text { Prospective } \\
\text { cohort study }\end{array}$ & 194,711 & Physical activity & $\begin{array}{l}\text { Inversely associated } \\
\text { with risk of } C D\end{array}$ & Khalili, H. & 61 \\
\hline & $\begin{array}{l}\text { Uncontrolled pilot } \\
\text { study }\end{array}$ & 12 & $\begin{array}{c}\text { 12-week walking } \\
\text { program }\end{array}$ & $\begin{array}{c}\text { Beneficial for IBD } \\
\text { patients } \\
\end{array}$ & Loudon, CP. et al. & 63 \\
\hline \multirow{6}{*}{$\begin{array}{c}\text { Sleep } \\
\text { disturbances }\end{array}$} & $\begin{array}{l}\text { Longitudinal, } \\
\text { internet-based } \\
\text { cohort data }\end{array}$ & 3173 & Questionnaire & $\begin{array}{l}\text { Increased risk of disease } \\
\text { flares in } C D \text { but not UC }\end{array}$ & $\begin{array}{l}\text { Ananthakrishnan, } \\
\text { AN. et al. }\end{array}$ & 75 \\
\hline & Clinical study & 10 & $\begin{array}{l}\text { Disturbances in sleep- } \\
\text { wake cycle }\end{array}$ & $\begin{array}{c}\text { Sleep disturbances led } \\
\text { to immunologic } \\
\text { alterations }\end{array}$ & Born, J. et al. & 76 \\
\hline & Clinical study & 47 & $\begin{array}{c}\text { Questionnaire } \\
\text { assessing sleep quality }\end{array}$ & $\begin{array}{l}\text { Impaired sleep quality is } \\
\text { associated with pediatric } \\
\text { IBD }\end{array}$ & $\begin{array}{l}\text { Mahlmann, L. et } \\
\text { al. }\end{array}$ & 77 \\
\hline & Clinical study & 32 & $\begin{array}{c}\text { Questionnaire } \\
\text { assessing sleep quality }\end{array}$ & $\begin{array}{l}\text { Impaired quality of life } \\
\text { in IBD }\end{array}$ & Keefer, L. et al. & 78 \\
\hline & $\begin{array}{l}\text { Prospective } \\
\text { observational } \\
\text { cohort study }\end{array}$ & 41 & $\begin{array}{l}\text { Pittsburgh sleep } \\
\text { quality index (PSQI) }\end{array}$ & $\begin{array}{c}\text { Strong association } \\
\text { between poor sleep } \\
\text { quality and IBD }\end{array}$ & Ali, T. et al. & 79 \\
\hline & $\begin{array}{l}\text { In-vivo mouse } \\
\text { study }\end{array}$ & 33 & $\begin{array}{l}\text { Diet and sleep } \\
\text { disturbances }\end{array}$ & $\begin{array}{c}\text { Circadian } \\
\text { disorganization impacts } \\
\text { intestinal microbiota }\end{array}$ & Voigt, RM. et al. & 80 \\
\hline
\end{tabular}

${ }^{*} \mathrm{CD}=$ Crohn's disease; GWAS = genome-wide association studies; NSAIDs = nonsteroidal anti-inflammatory drugs; OCP = oral contraceptive pill; SNP = single nucleotide polymorphism; UC = ulcerative colitis; UPS = ubiquitin protease system

To date, genome-wide association studies revealed more than 200 susceptibility gene loci in IBD [3943]. First, based on a model selection analysis, 163 susceptibility gene loci were identified, of which 23 and 30 loci were shown to be specific for ulcerative colitis and Crohn's disease, respectively [39]. These data were further completed with a more recent association study identifying 38 novel risk loci [40]. Based on another large genetic association study in IBD patients, it was shown that ten single nucleotide polymorphisms, in a total of four genes, were found to be significantly correlated with Crohn's disease [41]. Strongest correlations were found with CYLD, a de-ubiquitinating enzyme, pointing towards the ubiquitin proteasome system as a major contributor to IBD pathogenesis [41]. A more recent and very large study of 34,819 IBD patients investigating genotype-phenotype associations across 156,154 genetic variants also provided important insights into the genetic heterogeneity between ileal and colonic Crohn's disease, thereby rejecting the current classification of Crohn's disease versus ulcerative colitis [42].

In addition to genetics, the association between non-coding single nucleotide polymorphisms and IBD risk has gained major interest [44]. Moreover, based on several twin studies, the importance of 
non-genetic environmental factors $[45,46]$ on IBD disease manifestation has become clear. One example is the implication of micronutrients in IBD progression. Patients with IBD are commonly diagnosed with a vitamin $D$ deficiency [47], which can be related to lowered oral intake of vitamin D or decreased sunlight exposure. A more recent study in healthy volunteers showed that, specifically, the gut microbiome of the upper gastrointestinal tract is positively influenced in response to vitamin D3 treatment [48], suggesting that vitamin D plays a protective role in IBD pathogenesis. Alternatively, intracellular levels of zinc [49] and iron [50] have been associated with bacterial clearance and consequent intestinal permeability and increased risk of IBD, respectively. Another environmental, lifestyle-related factor is smoking, which has been shown to cause a two-fold increased risk for IBD [51]. Besides affecting the nicotinic acetylcholine receptors present on gastrointestinal mucosal epithelial cells [52], smoking can modulate the human gut microbiota composition, thereby affecting the course of the disease in IBD. Although in the context of Crohn's disease, smoking has adverse effects, in ulcerative colitis patients, it may play a protective role, implying that smoking may be a disease-specific modifier [53].

As mentioned, dietary fiber intake is able to prolong IBD remission through subsequent increase in luminal production of short-chain fatty acids $[31,54]$. In addition, it is well-established that high intake of fat- and sugar-enriched foods are capable of regulating intestinal microbiota composition and diversity [55,56], thereby also initiating and sustaining inflammation in patients with IBD [57]. Furthermore, high intake of $n-3$ polyunsaturated fatty acids and plant sterols have been shown to be protective [58], suggesting that dietary changes play a role in IBD pathogenesis.

It is also well known that low-to-moderate intensity exercise positively affects immune function [59]. Indeed, preclinical studies showed that moderate intensity exercise decreased the expression of proinflammatory cytokines, thereby improving acute colitis [60]. Human data regarding the beneficial effects of exercise on the development of intestinal inflammation are mixed, mainly due to the variations in type and rate of exercise. Several studies suggested an inversed correlation between physical activity and the risk or onset of IBD [61]; however, these effects have shown to be disease specific [62]. Nevertheless, other studies focusing on the association between exercise and disease course or the quality of life of IBD patients found a beneficial effect on well-being, sleep, confidence, and mood $[63,64]$.

Further evidence also suggests that geographic location and socioeconomic status are associated with the risk of IBD, thereby supporting the "hygiene hypothesis" of Bloomfield [65]. This hypothesis postulates that the recent rapid rise in IBD, especially in industrialized regions [66], may be due to the lower rate of infection during childhood. The lower infection rate may evolve from reduced exposure to enteric bacteria and improved sanitation during early life [67]. Although this reasoning might indeed explain the higher incidence of IBD in urban areas, the environmental location has been shown to differently affect the prevalence of Crohn's disease and ulcerative colitis. Whereas Crohn's disease has shown to be more frequent in urban/coastal areas, ulcerative colitis is more prevalent in inland municipalities [68].

Given that the gut microbiota is relatively diverse and unstable during early childhood, any kind of alteration is likely to affect the intestinal immune responses and predispose individuals to IBD. For instance, medications, including antibiotics, contraceptives as well as non-steroidal antiinflammatory drugs (NSAIDs) are known to increase the risk of IBD, likely through altering the commensal flora and/or the intestinal barrier [69]. More specifically, based on a meta-analysis, 


\section{Chapter 5}

antibiotics were shown to associate with increased risk of new-onset Crohn's disease rather than ulcerative colitis [70]. In line, a multiple database search revealed that individuals exposed to oral contraceptives had a $24 \%$ and $30 \%$ increased risk for developing Crohn's disease and ulcerative colitis, respectively, compared with those not exposed to the medication [71]. Likewise, high doses and long-term treatments with NSAIDs [72] resulted in the exacerbation of IBD [73], potentially acting via non-selective inhibition of the cyclo-oxygenase [74].

Relevantly, IBD disease activity and its risk of relapse has also been associated with sleep disturbances [75]. Sleep disturbances can induce the levels of inflammatory cytokines, thereby activating an inflammatory cascade [76]. Furthermore, sleep disturbances have been shown to occur in IBD patients, including pediatric patients [77] as well as those with inactive disease [78], and can negatively impact quality of life. Indeed, optimized sleep duration (i.e., six to nine hours/day) was able to decrease the risk of ulcerative colitis. Further, based on a prospective study, a strong inversed correlation between sleep quality and the activity of IBD was demonstrated [79]. Also, disruptions of the circadian organization, a form of long-term biological stress, are known to affect health [80]. It has been suggested that the adverse effects of the host's circadian rhythm, including sleep disruption, can alter the circadian rhythm of the intestinal microbiota, thereby changing its community structure [80]. Given that the gut microbiota plays a key role in the development of IBD, it is also likely that circadian disorganization, through dysbiosis of the intestinal microbiota, negatively impacts the course of the disease.

\section{Stress and intestinal microbiota: Bidirectional relationship in IBD}

\subsection{Influence of Stress on Gut Microbiota}

Several lines of evidence suggest that stress, induced by dietary, environmental or neuroendocrine factors, can adversely affect the gut/microbiota/brain axis [81,82] (Table 2).

Table 2: Studies investigating the link between stress and gut microbiota

\begin{tabular}{|c|c|c|c|c|c|c|}
\hline Factor & Type of study & $\mathbf{N}$ & $\begin{array}{l}\text { Intervention/ } \\
\text { Methodology }\end{array}$ & Outcome & Author(s) & Reference \\
\hline \multirow{6}{*}{$\begin{array}{c}\text { Prenatal/early life } \\
\text { stress }\end{array}$} & $\begin{array}{l}\text { In-vivo mouse } \\
\text { study }\end{array}$ & $\begin{array}{l}\text { 6-20 mice } \\
\text { per group }\end{array}$ & Maternal high-fat diet & $\begin{array}{l}\text { Dysbiosis and low-grade } \\
\text { inflammation in the } \\
\text { intestine }\end{array}$ & Xie, R. et al. & 83 \\
\hline & In-vivo rat study & $\begin{array}{l}\text { 6-10 per } \\
\text { group }\end{array}$ & Prenatal stress & $\begin{array}{l}\text { Long-lasting alterations } \\
\text { in the intestinal } \\
\text { microbiota composition }\end{array}$ & $\begin{array}{c}\text { Golubeva, AV. et } \\
\text { al. }\end{array}$ & 84 \\
\hline & $\begin{array}{l}\text { In-vivo mouse } \\
\text { study }\end{array}$ & $\begin{array}{l}\text { 21-23 mice } \\
\text { per group }\end{array}$ & Prenatal stress & $\begin{array}{c}\text { Alterations in vaginal } \\
\text { microbiota contributed } \\
\text { to reprogramming of the } \\
\text { developing brain }\end{array}$ & Jasarevic, E. et al. & 85 \\
\hline & $\begin{array}{c}\text { In vivo primates } \\
\text { study }\end{array}$ & 20 & Maternal separation & $\begin{array}{l}\text { Maternal separation- } \\
\text { induced psychological } \\
\text { disturbances altered } \\
\text { intestinal microflora }\end{array}$ & Bailey, MT. et al. & 91 \\
\hline & In-vivo rat study & 22 & Maternal separation & $\begin{array}{l}\text { Early life stress induced } \\
\text { alterations in gut-brain } \\
\text { axis contributing to IBD } \\
\text { symptoms }\end{array}$ & $\begin{array}{c}\text { O'Mahony, SM. et } \\
\text { al. }\end{array}$ & 92 \\
\hline & $\begin{array}{l}\text { Longitudinal } \\
\text { clinical study }\end{array}$ & $\begin{array}{c}192 \\
\text { children }\end{array}$ & Questionnaire & $\begin{array}{l}\text { Prenatal stress is } \\
\text { associated with } \\
\text { microbial colonization } \\
\text { patterns in infants }\end{array}$ & $\begin{array}{c}\text { Zijlmans, MA. et } \\
\text { al. }\end{array}$ & 104 \\
\hline
\end{tabular}


Inflammatory Bowel Disease: A stressed "gut/feeling"

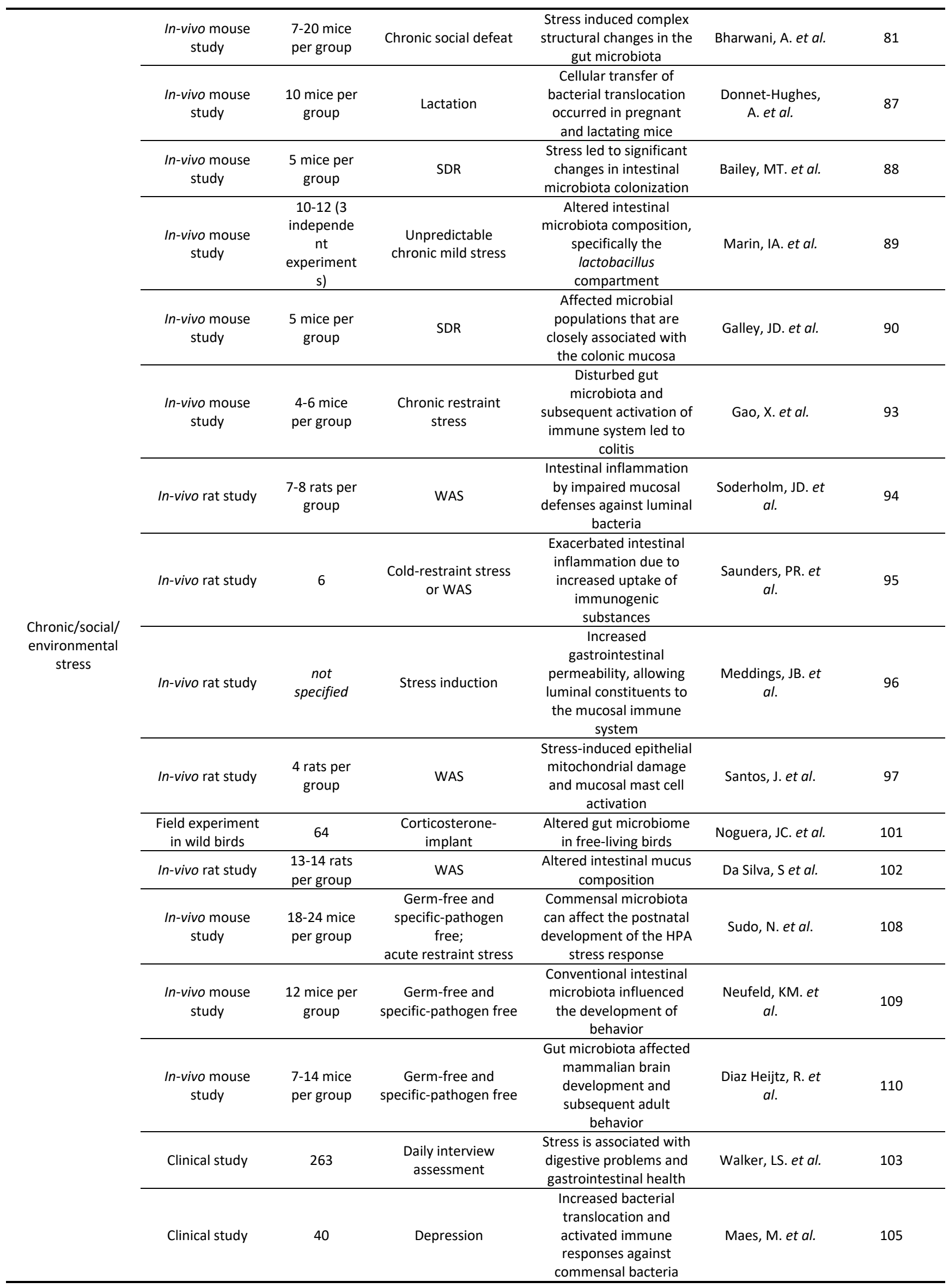




\begin{tabular}{|c|c|c|c|c|c|c|}
\hline & Clinical study & 65 & $\begin{array}{l}\text { Coping style } \\
\text { instrument }\end{array}$ & $\begin{array}{l}\text { IBD adolescents used } \\
\text { more avoidant coping } \\
\text { styles compared to } \\
\text { healthy controls }\end{array}$ & $\begin{array}{l}\text { Van der Zaag- } \\
\text { Loonen, HJ. et al. }\end{array}$ & 106 \\
\hline \multirow{7}{*}{ Pro/prebiotics } & In-vivo rat study & $\begin{array}{l}\text { 4-5 rats per } \\
\text { group }\end{array}$ & WAS and probiotics & $\begin{array}{c}\text { Probiotics prevented } \\
\text { chronic stress-induced } \\
\text { intestinal abnormalities }\end{array}$ & Zareie, M. et al. & 99 \\
\hline & In-vivo rat study & 84 & $\begin{array}{c}\text { Maternal separation } \\
\text { and } \\
\text { prebiotics/probiotics/ } \\
\text { LC-PUFA } \\
\end{array}$ & $\begin{array}{l}\text { Nutritional intervention } \\
\text { at weaning normalized } \\
\text { gut permeability and } \\
\text { restored growth rate }\end{array}$ & $\begin{array}{l}\text { Garcia-Rodenas, } \\
\text { CL. et al. }\end{array}$ & 100 \\
\hline & $\begin{array}{l}\text { In-vivo mouse } \\
\text { study }\end{array}$ & 36 & Probiotic formulation & $\begin{array}{l}\text { Suggested the } \\
\text { importance of probiotics } \\
\text { in gut/brain axis in } \\
\text { stress-related disorders }\end{array}$ & Bravo, JA. et al. & 111 \\
\hline & $\begin{array}{l}\text { In-vivo mouse } \\
\text { study }\end{array}$ & $\begin{array}{l}8 \text { mice per } \\
\text { group }\end{array}$ & $\begin{array}{l}\text { Chronic mild stress } \\
\text { and probiotics }\end{array}$ & $\begin{array}{l}\text { Decreased pro- } \\
\text { inflammatory cytokines } \\
\text { and altered stress- } \\
\text { related behaviors }\end{array}$ & Li, N. et al. & 113 \\
\hline & In-vivo rat study & 36 rats; & Probiotic formulation & $\begin{array}{c}\text { Anxiolytic-like activity in } \\
\text { rats }\end{array}$ & $\begin{array}{c}\text { Messaoudi, M. et } \\
\text { al. }\end{array}$ & 112 \\
\hline & $\begin{array}{l}\text { Double-blind, } \\
\text { placebo- } \\
\text { controlled, } \\
\text { randomized } \\
\text { parallel group } \\
\text { study }\end{array}$ & $\begin{array}{c}66 \\
\text { individuals }\end{array}$ & Probiotic formulation & $\begin{array}{c}\text { Beneficial psychological } \\
\text { effects in healthy human } \\
\text { volunteers }\end{array}$ & $\begin{array}{c}\text { Messaoudi, M. et } \\
\text { al. }\end{array}$ & 112 \\
\hline & Systematic review & 11 RCTs & $\begin{array}{c}\text { Prebiotic } \\
\text { supplementation }\end{array}$ & $\begin{array}{l}\text { Short-term beneficial } \\
\text { effects in intestinal } \\
\text { microbiota composition }\end{array}$ & Rao, S. et al. & 114 \\
\hline
\end{tabular}

* HPA = hypothalamic pituitary adrenal axis; LC-PUFA = long-chain poly-unsaturated fatty acids; RCT = randomized controlled trials; SDR = social disruption; WAS = water-avoidance stress

\subsubsection{Preclinical Studies}

It was recently shown in mice that maternal nutrition can negatively affect offspring intestinal development and function. For instance, maternal high-fat diet caused a shift in microbiota composition, thereby predisposing the offspring to develop intestinal inflammation [83]. In line, early prenatal stress in rodents was shown to increase the Oscillibacter, Anaerotruncus, and Peptococcus genera [84] and induce a loss of Lactobacilli transmission to the neonate $[84,85]$, pointing towards the involvement of birth canal delivery in gut microbiota colonization $[86,87]$. These data were further supported by other preclinical studies showing that short-term or mild chronic stress caused a reduction in Lactobacilli [88-90]. These data suggest that stressor-induced changes have important health implications [90]. It has also been shown, both in primates [91] and in rodents [92], that maternal separation, a form of chronic stress, was able to induce a change in fecal microbiota in newborn animals. A more recent study demonstrated that chronic stress resulted in dysbiosis of the murine gut microbiota, thereby inducing an immune system response and facilitating experimentally induced colitis [93]. Other genetically susceptible rodent models revealed that chronic psychological stress induced mucosal dysfunction, intestinal abnormalities, and subsequently intestinal inflammation [94]. Likewise, it was shown in rats that acute [95], environmental [96] as well as chronic stress [97] increased intestinal permeability, and hence, luminal molecule delivery to the mucosal immune system, thereby triggering pro-inflammatory responses. In this context, probiotics (living organisms yielding benefits on the host's microbiome [98]) were shown to revert chronic stress-induced abnormalities of the intestinal tract [99]. Also, it was shown that diets containing a combination of specific long-chain polyunsaturated fatty acids, prebiotics, and probiotics restored 
the rat intestinal microbiota composition [100]. More recently, it was even demonstrated that stress hormones, through manipulation of basal corticosterone levels, were able to alter the gut microbiome of free-living birds [101]. Further, using a sophisticated rat model, water avoidance stress was shown to alter the mucus composition [102], which is known as the host's primary innate defense. Given that changes in the production of mucosal proteins have been associated with dysbiosis of the gut microbiota, it is likely that stress indirectly affects the intestinal microbiota via inflammation of the mucosal protein layer [94].

\subsubsection{Clinical studies}

Few clinical studies also revealed that stress is associated with digestive problems and gastrointestinal health [103]. For instance, by means of a phylogenetic microarray, one study showed that exposure to stress during pregnancy resulted in aberrant microbiota colonization patterns in pediatrics, which likely increased inflammation and gastrointestinal symptoms [104]. In line with these findings, stress-related psychiatric disorders, such as depression, were also associated with increased bacterial translocation, thereby activating immune responses against commensal bacteria [105]. Although these data imply that stress has a potent influence on intestinal microbiota, stress is a subjective experience, which makes it challenging to objectively evaluate the effects of stress. Therefore, further human studies should be performed to verify that stress results in dysbiosis of the gut microbiota.

\subsection{Impact of intestinal microbiota on stress responsiveness}

Appropriate physiological responses to stress and/or immunity are necessary for survival. As such, aberrant responsiveness can be detrimental to the host, leading to the development of chronic disorders, including IBD [106] and brain disorders [107].

\subsubsection{Preclinical studies}

Preclinical studies using germ-free animals, specific pathogen-free animals, and animals exposed to pathogens, probiotics or antibiotics have been performed to better understand how gut microbiota can regulate stress response, cognition, and behavior [28]. Previously, it has become clear that intestinal colonization with conventional microbiota at an early developmental stage is important for stress responsiveness in adult mice [108]. This study showed that the HPA stress response was exaggerated in the absence of normal gut microbiota reconstitution, whereas it could be partially corrected by reconstitution of feces from specific pathogen-free mice at an early, but not at a later stage. In line with these results, other murine studies demonstrated the influence of conventional gut microbiota on the development of behavior $[109,110]$, and showed that this effect occurred along with neurochemical changes in the brain [109]. Another study based on a rat model of acute psychological stress demonstrated that the probiotic Lactobacillus farciminis reduced intestinal leakiness, thereby decreasing plasma levels of lipopolysaccharides, and consequently, diminishing the HPA axis response to stress. Moreover, by reducing stress-induced plasma corticosterone levels, Bravo et al. [111] showed that the probiotic Lactobacillus rhamnosus was able to reduce the stress response as well as anxiety-related behaviors and cognition in mice. Furthermore, it has been shown 
that the combination of probiotics, such as Lactobacillus helveticus and Bifidobacterium longum, resulted in a reduction of anxiety-like behaviors in rodents $[112,113]$.

\subsubsection{Clinical studies}

Messaoudi et al. [112] also validated their preclinical findings in healthy human volunteers. Their findings suggested that probiotic formulation attenuated psychological distress in healthy volunteers, which may be linked to decreased urinary free cortisol levels [112]. In agreement with these data, a randomized, double-blind, placebo-controlled trial suggested that the administration of probiotics helped in reducing anxiety-like behavior among patients with chronic fatigue syndrome [114]. Altogether, these data further confirm that the intestinal microbiota plays a role in controlling stress responsiveness, behavior, and cognition.

\subsection{Stress and its impact on inflammation}

While not having discussed in detail yet, the impact of stress on the immune system appears to be quite complex. Depending on the type of stress (short-term or chronic) and/or hormones being released, a stressor may either suppress or enhance immune function [115].

\subsubsection{Preclinical studies}

Several preclinical studies have shown that short-term stress induces significant changes in absolute numbers and composition of blood leukocytes $[116,117]$. Likewise, short-term stress was shown to increase the circulating levels of interleukin-6 (IL6) and pro-inflammatory monocyte chemotactic protein-1 (MCP1/CCL2) [118]. These findings were also further confirmed by others showing that social disruption reduced microbial diversity and richness in mice, which correlated with increased circulating levels of the pro-inflammatory cytokines MCP1 and IL6 [88]. Relevantly, based on data demonstrating that administration of antibiotics was able to abolish social stress-mediated increases in pro-inflammatory cytokines [88], it is likely that intestinal microbiota plays a role in stressorinduced pro-inflammatory responses.

\subsubsection{Clinical studies}

Previous studies in humans provided similar evidence that stress induces an increase of proinflammatory Th1 cytokines [119-121]. For instance, academic stress, referred to as the body's response to academic-related workload that goes beyond the adaptive capabilities of students [122], was shown to significantly increase the production of interferon-gamma (IFN $\gamma$ ) and tumor necrosis factor alpha (TNF $\alpha$ ) [119]. Acute stress can also upregulate anti-inflammatory cytokines including IL10, while independently inhibiting pro-inflammatory cytokines such as TNF $\alpha$ [123]. These effects can induce a shift towards Th2-mediated humoral response [124], which may be essential to prevent hyperactivation of the stress system. Alternatively, chronic stress is known to increase the release of cortisol levels for several days, an effect that may be associated with immunosuppression, as shown by a reduction in circulating CD8+ lymphocytes, natural killer cells, and macrophages [125]. Nevertheless, as chronic psychological stress was also associated with increased levels of serum C- 
reactive protein (CRP) [126], these data suggest that chronic stress may also exert pro-inflammatory effects. Obviously, it should be noted that the gastrointestinal tract per se, including its local microbiota, may serve as an essential organ mediating immune responses [127]. This is not only of relevance in the context of human IBD, but also in irritable bowel syndrome (IBS) [128] as well as major depressive disorders [129].

\subsection{Stress and inflammation in IBD}

Inflammatory bowel disease is a complex disease that likely does not only consist of Crohn's disease and ulcerative colitis. Extensive translational research has been conducted to better understand the role of stress and inflammation in IBD [130].

\subsubsection{Preclinical studies}

Previously, using rodent models of spontaneous colitis, it was shown that intestinal inflammation is associated with defects in mucosal barrier or dysfunctional regulatory T lymphocytes [131]. Other studies using mice fed a dextran-sulfate-sodium diet to induce colitis revealed the importance of intestinal adhesion molecules, such as ICAM-1, in the development of intestinal inflammation [132]. Relevantly, when dextran-sulfate-sodium-treated mice were injected with enterotoxigenic Bacteroides fragilis, increased colitis and colonic inflammation was observed [133]. Similarly, in cellular models, Clostridium difficile toxin A was shown to induce apoptosis and inflammation in enterocytes [134,135]. Moreover, using a mouse model of depression, it was shown that stressinduced release of corticosteroids can reactivate IBD [136], likely via increased production of proinflammatory cytokines. These data imply that stress may affect the course of the disease.

\subsubsection{Clinical studies}

In humans, it was shown that infections with Bacteroides fragilis, through secretion of its proinflammatory toxin, is associated with development of ulcerative colitis [137]. Likewise, infections with Clostridium difficile is thought to be involved in the reactivation of IBD in patients [138]. Within a similar context, it was also shown that patients with ileal Crohn's disease had a higher percentage of invasive Escherichia coli in the mucosa as compared to healthy controls, and these percentages even correlated with severity of the disease [139]. In line, another study demonstrated that Escherichia coli can replicate inside macrophages of patients with Crohn's disease and subsequently secrete large amounts of TNF $\alpha$, thereby contributing to inflammation [140]. Collectively, these data point towards the strong relationship between the gut microbiota composition and intestinal inflammation.

It is also important to note that psychosocial stress, including psychological distress, anxiety, and depression, can induce low-grade chronic inflammation in the gut. For instance, in humans, it was shown that depression correlated with elevated levels of TNF $\alpha$ and CRP, pro-inflammatory cytokines that that are known to trigger inflammation in patients with IBD [141,142]. Hence, it has been proposed that stress gradually contributes to the development or exacerbation of IBD (as reviewed elsewhere $[28,143])$. Vice versa, compared to the general population, young patients with IBD 


\section{Chapter 5}

displayed higher rates of psychological stress, and these results were further confirmed in gastrointestinal disorders such as IBS $[144,145]$. Together, these data imply that the disease itself may have a direct impact on the quality of life of patients.

Yet, it is not clear whether individuals with higher stress also experience more IBD symptoms. A large cross-sectional, population-based study of IBD patients showed that the relationship between intestinal inflammation and symptomatic disease activity differed between Crohn's disease and ulcerative colitis [146]. Whereas Crohn's disease did not associate with intestinal inflammation and disease activity symptoms, an association was found for ulcerative colitis. These findings suggest that the duration and intensity of stress factors may have differential influences on chronic inflammatory diseases. Furthermore, this study showed that perceived stress in both diseases correlated with disease activity symptoms, while not with inflammation [146]. Although the majority of conventional therapies in IBD focus on tackling intestinal inflammation, these insights open new venues for stress reduction in the management of IBD.

\section{Managing stress in IBD: Does it make the gut feel better?}

In recent years, significant progress has been made in the treatment of IBD, focusing either on targeted therapies [147] or on alternative and complementary strategies [148], which has recently been extensively reviewed $[147,148]$. Nevertheless, there is no certain cure for IBD due to the limited effectiveness of current therapies, which often even goes hand in hand with significant side effects. Quality of life as well as anxiety and depression are known predictors of negative medical outcomes in many chronic conditions, and as reviewed, stress has a profound impact on these variables in patients with IBD. Hence, a number of approaches have focused on relieving stress as a potential therapeutic option in IBD (Table 3) [148,149].

Table 3: Clinical studies investigating stress management in IBD

\begin{tabular}{|c|c|c|c|c|c|c|}
\hline Factor & Type of study & $\mathbf{N}$ & Intervention & $\begin{array}{c}\begin{array}{c}\text { Outcome in IBD } \\
\text { patients }\end{array} \\
\end{array}$ & Author(s) & Reference \\
\hline \multirow{2}{*}{$\begin{array}{l}\text { Guided imagery / } \\
\text { Relaxation } \\
\text { training }\end{array}$} & Pilot RCT & 28 & $\begin{array}{l}\text { Guided imagery with } \\
\text { relaxation (GIR) }\end{array}$ & $\begin{array}{c}\text { Improved QL in elderly } \\
\text { women with } \\
\text { osteoarthritis }\end{array}$ & Baird, CL. et al. & 150 \\
\hline & Prospective RCT & 39 & Relaxation-training & $\begin{array}{c}\text { Beneficial effects on } \\
\text { anxiety, pain and stress } \\
\text { in IBD patients }\end{array}$ & Mizrahi, MC. et al. & 163 \\
\hline \multirow{3}{*}{$\begin{array}{c}\text { Lifestyle } \\
\text { management }\end{array}$} & Clinical study & 60 & $\begin{array}{l}\text { 60-hour training } \\
\text { program in lifestyle } \\
\text { modification } \\
\text { over a period of } 10 \\
\text { weeks }\end{array}$ & $\begin{array}{l}\text { Short-term benefits in } \\
\text { the QL in UC patients, } \\
\text { whereas no long-term } \\
\text { effects }\end{array}$ & Langhorst, J. et al. & 157 \\
\hline & Clinical study & 49 & $\begin{array}{c}\text { 8-session information } \\
\text { about QL and stress } \\
\text { management }\end{array}$ & $\begin{array}{l}\text { No effect on anxiety } \\
\text { levels } 6 \text { months post- } \\
\text { intervention }\end{array}$ & Larsson, K. et al. & 160 \\
\hline & $\begin{array}{l}\text { Prospective, } \\
\text { randomized } \\
\text { waiting-control } \\
\text { group design }\end{array}$ & 30 & $\begin{array}{l}\text { 60-hour training } \\
\text { program on lifestyle } \\
\text { management }\end{array}$ & $\begin{array}{l}\text { Improved QL in patients } \\
\text { with UC remission }\end{array}$ & $\begin{array}{c}\text { Elsenbruch, S. et } \\
\text { al. }\end{array}$ & 167 \\
\hline
\end{tabular}


Inflammatory Bowel Disease: A stressed "gut/feeling"

\begin{tabular}{|c|c|c|c|c|c|c|}
\hline & $\begin{array}{l}\text { Prospective, } \\
\text { randomized study }\end{array}$ & 32 & $\begin{array}{l}\text { Low-intensity walking } \\
\text { program }\end{array}$ & $\begin{array}{c}\text { Improved QL of CD } \\
\text { patients }\end{array}$ & $\mathrm{Ng}$, V. et al. & 179 \\
\hline & Prospective RCT & 30 & $\begin{array}{c}\text { Moderate-intensity } \\
\text { running } \\
\end{array}$ & $\begin{array}{c}\text { Improved QL of IBD } \\
\text { patients }\end{array}$ & Klare, P. et al. & 180 \\
\hline \multirow{9}{*}{ Psychotherapy } & Two clinical trials & 36 & $\begin{array}{l}\text { 7-session behavioral } \\
\text { protocol }\end{array}$ & $\begin{array}{l}57 \% \text { reduction in IBD } \\
\text { relapse in the following } \\
12 \text { months }\end{array}$ & Keefer, L. et al. & 152 \\
\hline & $\mathrm{RCT}$ & 41 & $\begin{array}{c}\text { Primary and } \\
\text { Secondary Control } \\
\text { Enhancement } \\
\text { Therapy-Physical } \\
\text { Illness } \\
\end{array}$ & $\begin{array}{l}\text { Beneficial effects on } \\
\text { depression in IBD } \\
\text { adolescents }\end{array}$ & Szigethy, E. et al. & 158 \\
\hline & Clinical study & 178 & Nurse-led counselling & $\begin{array}{l}\text { Improved QL over } 6 \\
\text { rather than } 12 \text { months } \\
\text { in IBD patients }\end{array}$ & Smith, GD. et al. & 161 \\
\hline & $\begin{array}{c}\text { Prospective, } \\
\text { uncontrolled open } \\
\text { trial }\end{array}$ & 30 & $\begin{array}{l}\text { Supportive-expressive } \\
\text { group psychotherapy }\end{array}$ & $\begin{array}{c}\text { No changes in } \mathrm{QL}, \\
\text { anxiety, or depression } \\
\text { over the course of } \\
\text { treatment in } \mathrm{UC} / \mathrm{CD} \\
\end{array}$ & $\begin{array}{c}\text { Maunder, RG. et } \\
\text { al. }\end{array}$ & 162 \\
\hline & Meta-analysis & $\begin{array}{c}1824 \\
\text { studies } \\
\text { with } 14 \\
\text { RCTs } \\
\end{array}$ & Psychological therapy & $\begin{array}{c}\text { Small short-term } \\
\text { beneficial effects on QL } \\
\text { and depression in IBD } \\
\text { patients }\end{array}$ & Gracie, DJ. et al. & 166 \\
\hline & $\mathrm{RCT}$ & 29 & $\begin{array}{c}\text { Breath-Body-Mind } \\
\text { Workshop; } \\
\text { questionnaire }\end{array}$ & $\begin{array}{l}\text { Significant long-lasting } \\
\text { benefits for IBD } \\
\text { symptoms, anxiety, } \\
\text { depression and QL } \\
\end{array}$ & Gerbarg, PL. et al. & 155 \\
\hline & Control study & 60 & $\begin{array}{l}\text { Mindfulness-based } \\
\text { stress reduction }\end{array}$ & $\begin{array}{l}\text { Improved mood and QL } \\
\text { after six months of } \\
\text { intervention }\end{array}$ & Neilson, K. et al. & 156 \\
\hline & RCT & 36 & $\begin{array}{l}\text { Gut-directed } \\
\text { hypnotherapy }\end{array}$ & $\begin{array}{c}\text { Gut-directed } \\
\text { hypnotherapy may be } \\
\text { one aspect in a disease- } \\
\text { management program } \\
\text { for IBD }\end{array}$ & Keefer, L. et al. & 159 \\
\hline & Clinical trial & 66 & $\begin{array}{c}\text { Multi-convergent } \\
\text { therapy } \\
\text { (psychotherapy) }\end{array}$ & $\begin{array}{c}\text { Therapy is beneficial in } \\
\text { the management of IBD } \\
\text { symptoms }\end{array}$ & Berrill, JW. et al. & 164 \\
\hline \multirow{6}{*}{ Medication } & $\begin{array}{c}\text { Retrospective } \\
\text { observational study }\end{array}$ & 30 & Herbal treatment & $\begin{array}{c}\text { Positive effect of } \\
\text { cannabis on disease } \\
\text { activity in CD }\end{array}$ & Naftali, T. et al. & 171 \\
\hline & $\begin{array}{c}\text { Prospective, } \\
\text { placebo-controlled } \\
\text { study }\end{array}$ & 21 & Herbal treatment & $\begin{array}{c}\text { Short course of cannabis } \\
\text { had beneficial effects in } \\
\text { CD patients }\end{array}$ & Naftali, T. et al. & 172 \\
\hline & Double-blind RCT & 108 & Placebo or vitamin D3 & $\begin{array}{c}\text { Reduced relapse risk in } \\
\text { CD } \\
\end{array}$ & $\begin{array}{c}\text { Jorgensen, SP. et } \\
\text { al. }\end{array}$ & 174 \\
\hline & Prospective & 37 & $\begin{array}{l}\text { Active or plain vitamin } \\
\text { D }\end{array}$ & $\begin{array}{c}\text { Active form of vitamin D } \\
\text { has short-term } \\
\text { beneficial effects in } C D\end{array}$ & Miheller, P. et al. & 175 \\
\hline & Meta-analysis & 12 studies & $\begin{array}{l}\text { Serum folate and } \\
\text { vitamin B12 }\end{array}$ & $\begin{array}{l}\text { Low concentration of } \\
\text { serum folate is a risk } \\
\text { factor for IBD and } \\
\text { supplementation may } \\
\text { be beneficial }\end{array}$ & Pan, Y. et al. & 177 \\
\hline & Double-blind RCT & $\begin{array}{l}10 \text { per } \\
\text { group }\end{array}$ & $\begin{array}{c}\text { Placebo/ } \\
\text { phylloquinone/ } \\
\text { vitamin D3 }\end{array}$ & $\begin{array}{l}\text { No significant beneficial } \\
\text { effects of phylloquinone } \\
\text { on bone health in } C D \\
\text { patients }\end{array}$ & $\begin{array}{c}\text { O'Connor EM. et } \\
\text { al. }\end{array}$ & 178 \\
\hline
\end{tabular}

* $\mathrm{CD}=$ Crohn's disease; $\mathrm{QL}=$ quality of life; $\mathrm{RCT}=$ randomized controlled trial; $\mathrm{UC}=$ ulcerative colitis

Stress management is a technique used to diminish the physiological effects of stress and tension, and to help the individual to improve his/her coping skill. One variant of such therapy is relaxation, by which the individual is trained by a therapist or in a self-directed manner to create physiological and mental rest. Several studies showed the effectiveness of relaxation training in a variety of physical illnesses, including cardiovascular disease, arthritis [150], and IBD [151-153]. Previously, it was shown that stress management could significantly improve IBD symptoms such as pain and 
fatigue [151]. In line, behavioral self-management therapy resulted in a 57\% decrease in 1 year risk to relapse in IBD patients [152], supporting the beneficial effects of stress management in IBD. More recently, two clinical studies pointed towards the beneficial effects of mindfulness on psychological and physical symptoms, quality of life, and C-reactive protein, an established biomarker [154], in patients with IBD $[155,156]$. However, based on a systematic review, McCombie et al. [153] concluded that the effect of psychotherapy led to inconsistent results in IBD patients. It should be noted that the studies included in this study focused on a wide range of therapies. Thus, although meditation and relaxation may have beneficial effects on inflammatory activity and quality of life in IBD patients, the effectiveness of mindfulness-based interventions on disease activity remains to be elucidated.

Whereas several studies demonstrated that psychotherapy had a beneficial impact on anxiety [157], depression [158], and quality of life of IBD patients [159], other studies were not able to find any effect $[160,161]$. Yet, two other studies investigated the impact of combining a variety of techniques as a treatment option in IBD $[161,162]$. Indeed, when combining relaxation with guided imagery, a method focusing on mind-relaxing images to replace stressful thoughts, both anxiety status and quality of life appeared to be improved among patients with IBD [163]. Similarly, multi-convergent therapy, which combines mindfulness meditation with cognitive behavioral therapy, has been used as a therapeutic option in patients with tinnitus and IBS. Therefore, its applicability and efficacy were investigated in an IBD population that received conventional therapy [164]. This study revealed that multi-convergent therapy improved quality of life mainly in IBD patients suffering from IBS-like symptoms [164], suggesting that this strategy has beneficial effects only in a subgroup of IBD patients. Although IBS and IBD are medically distinct from each other, symptoms compatible with IBS indeed often co-exist in patients with IBD [165], and should therefore not be underestimated. Within this context, it was recently shown that $36 \%$ and $37 \%$ of CD and UC patients, respectively, met IBS diagnostic criteria [165], confirming that the presence of IBS-like symptoms in IBD is common [148]. Furthermore, patients with IBS in quiescent IBD were shown to have significantly more anxiety and depression than patients without IBS [165].

More recent data suggested that short-term cognitive behavioral therapy improved quality of life and depression scores in patients with IBD, while not affecting disease activity or other measures of psychological well-being [166]. Others particularly investigated the impact of mind-body therapy, a combination of moderate exercise, diet, stress management training, behavioral techniques and selfcare strategies on patients with ulcerative colitis. Results suggested that this approach has a positive effect on IBD development by improving quality of life and mental/physical health scores [167].

As reviewed in detail elsewhere, other complementary and alternative medicines, such as herbal medicine, vitamin supplementation, and exercise have also gained attention for its anti-inflammatory properties and usefulness in the treatment of IBD $[148,168]$. Within this context, preclinical data from mice suggested that cannabinoid receptor activation mediates protective mechanisms in experimental colitis $[169,170]$. In line with these data, two clinical studies reported that cannabis was able to reduce IBD symptoms [171,172], pointing towards its ability to treat IBD. Nevertheless, whether cannabis is able to positively affect the course of disease requires further investigation. Other preclinical studies in mice showed an important role for vitamin $D$ and its receptor in the regulation of inflammation of the gastrointestinal tract [173]. Indeed, IBD patients often lack this vitamin [47], and hence, studies investigated the role of vitamin D [174-176] in treatment of IBD 
patients. Although these studies pointed towards beneficial effects on disease activity and risk of relapse, other clinical studies on the use of vitamin B [177] or K [178] in IBD treatment were inconsistent. These data imply that there is a lack of evidence to support positive effects of vitamins on IBD disease course [148]. Likewise, whereas preclinical [60] and clinical studies [179,180] suggested that low-to-moderate intensity exercise exerted beneficial effects on intestinal inflammation, overall health, and quality of life of IBD patients, further studies investigating the impact of exercise on disease activity and/or prevention of IBD are warranted [148].

Given that stress orchestrates an important influence on structural and functional aspects of the microbiome, multiple studies have also investigated the role of psychobiotics in stress-related diseases. Psychobiotics refer to probiotics or prebiotics that can manipulate commensal gut microbiota, and when ingested at adequate quantities, may indirectly have positive psychiatric effects in psychopathology [181]. As extensively reviewed, both in experimental colitis and human IBD, pre- and probiotics have shown beneficial effects in the prevention of IBD by modulating the trophic functions of the microbiota, improving the intestinal mucosal barrier and mediating antiinflammatory responses [182]. Given that the intake of psychobiotics also seem to exert antidepressant effects, including improvements in mood and decreases in stress-related plasma and urinary free cortisol [181], it may be postulated that psychobiotics might serve as therapeutic modulators of the gut/microbiota axis and positively influence psychological functions in the context of IBD.

Altogether, the quality of life and course of IBD are regulated by psychological conditions, and as such, the implementation of stress management may play an important role in IBD disease regression (see also Figure 1). Nevertheless, current studies are limited in sample size and study design, and hence, may lack proper controls. Therefore, future research is needed to validate current treatment options and/or to explore novel therapeutic opportunities in order to prevent IBD onset or improve stress-affected well-being of IBD patients. 


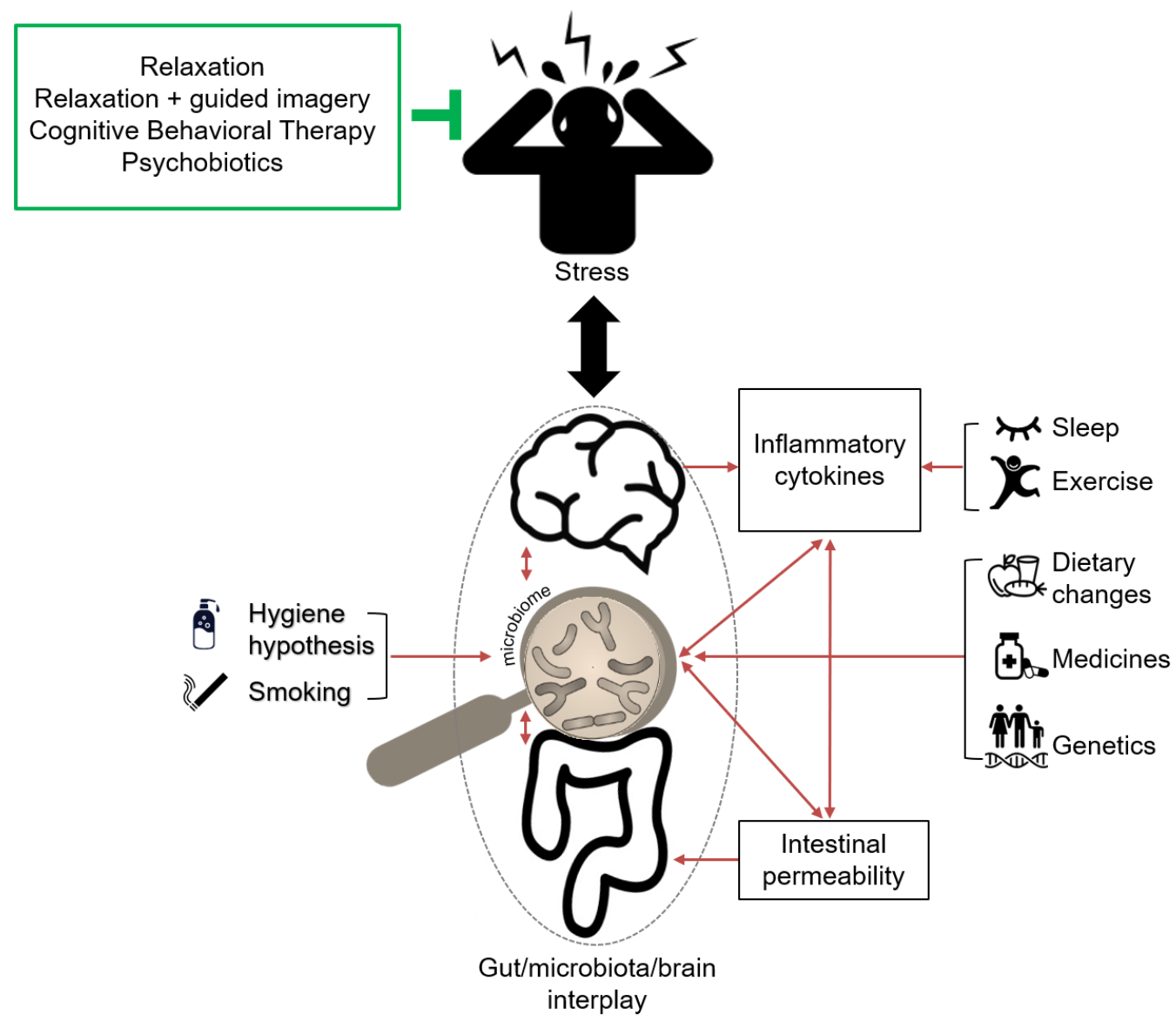

Figure 1 The gut/microbiota/brain interplay and its interactions upon exposure to stress. Under conditions of psychological stress including lack of sleep and physical inactivity, the brain (HPA axis) stimulates the production of pro-inflammatory cytokines. This can result in increased intestinal permeability and altered gut microbiota. In addition, fat- and sugar-enriched foods, long-term usage of medicines, as well as genetic predisposition can directly affect the gut microbiota composition, and subsequently, intestinal permeability. Furthermore, personal habits, such as hygiene and smoking, can also have an impact on the gut microbiome. Altogether, the multitude of stress-related factors can perturb the gut/microbiota/brain interplay, which contributes to the development of IBD. Relevantly, several stress management techniques have been proven to greatly alleviate IBD symptoms and improve the quality of life of IBD patients. Given that the exact underlying mechanisms in the context of IBD are not yet fully understood, therapeutic options aimed at improving stress management deserve further investigation. 


\section{Conclusion}

In summary, this review summarized the tight connection between the gut, microbiota, and brain in the context of IBD and has particularly shed light on the impact of stress on this interplay. It should be noted, however, that it is rather challenging to investigate the impact of stress on IBD, as stress can arise from totally different origins and may be closely connected to potential individual confounding factors, including (mental) health status and inter-individual variability in stress responsiveness and/or vulnerability. Therefore, future research involving preclinical studies as well as large-scale, controlled clinical trials should not only focus on unravelling the exact mechanisms through which stress affects IBD. However, it is also of great interest to further investigate the exact mechanisms of how stress management can orchestrate beneficial effects in IBD and how stressrelieving therapies should be implemented in IBD care. 


\section{References}

1. Tontini, G.E.; Vecchi, M.; Pastorelli, L.; Neurath, M.F.; Neumann, H. Differential diagnosis in inflammatory bowel disease colitis: state of the art and future perspectives. World J. Gastroenterol. 2015, 21, 21-46, doi:10.3748/wjg.v21.i1.21.

2. Ananthakrishnan, A.N. Epidemiology and risk factors for IBD. Nat Rev. Gastroenterol Hepatol 2015, 12, 205-217, doi:10.1038/nrgastro.2015.34.

3. Ye, Y.; Pang, Z.; Chen, W.; Ju, S.; Zhou, C. The epidemiology and risk factors of inflammatory bowel disease. Int J. Clin. Exp. Med. 2015, 8, 22529-22542.

4. Camara, R.J.; Ziegler, R.; Begre, S.; Schoepfer, A.M.; von Kanel, R.; Swiss Inflammatory Bowel Disease Cohort Study, g. The role of psychological stress in inflammatory bowel disease: quality assessment of methods of 18 prospective studies and suggestions for future research. Digestion 2009, 80, 129-139, doi:10.1159/000226087.

5. Mawdsley, J.E.; Rampton, D.S. Psychological stress in IBD: new insights into pathogenic and therapeutic implications. Gut 2005, 54, 1481-1491, doi:10.1136/gut.2005.064261.

6. Yeager, M.P.; Pioli, P.A.; Guyre, P.M. Cortisol exerts bi-phasic regulation of inflammation in humans. Dose Response 2011, 9, 332-347, doi:10.2203/dose-response.10-013.Yeager.

7. Zheng, G.; Victor Fon, G.; Meixner, W.; Creekmore, A.; Zong, Y.; M, K.D.; Colacino, J.; Dedhia, P.H.; Hong, S.; Wiley, J.W. Chronic stress and intestinal barrier dysfunction: Glucocorticoid receptor and transcription repressor HES1 regulate tight junction protein Claudin-1 promoter. Sci. Rep. 2017, 7, 4502, doi:10.1038/s41598-017-04755-w.

8. Petrosus, E.; Silva, E.B.; Lay, D., Jr.; Eicher, S.D. Effects of orally administered cortisol and norepinephrine on weanling piglet gut microbial populations and Salmonella passage. J. Anim. Sci. 2018, 96, 4543-4551, doi:10.1093/jas/sky312.

9. Gonzalez-Ariki, S.; Husband, A.J. The role of sympathetic innervation of the gut in regulating mucosal immune responses. Brain Behav. Immun. 1998, 12, 53-63, doi:10.1006/brbi.1997.0509.

10. Mourad, F.H.; Saade, N.E. Neural regulation of intestinal nutrient absorption. Prog. Neurobiol. 2011, 95, 149-162, doi:10.1016/j.pneurobio.2011.07.010.

11. Cryan, J.F.; O'Mahony, S.M. The microbiome-gut-brain axis: from bowel to behavior. Neurogastroenterol. Motil. 2011, 23, 187-192, doi:10.1111/j.1365-2982.2010.01664.x.

12. Clapp, M.; Aurora, N.; Herrera, L.; Bhatia, M.; Wilen, E.; Wakefield, S. Gut microbiota's effect on mental health: The gut-brain axis. Clin. Pract. 2017, 7, 987, doi:10.4081/cp.2017.987.

13. Yoo, B.B.; Mazmanian, S.K. The Enteric Network: Interactions between the Immune and Nervous Systems of the Gut. Immunity 2017, 46, 910-926, doi:10.1016/j.immuni.2017.05.011.

14. Furness, J.B.; Costa, M. Types of nerves in the enteric nervous system. Neuroscience 1980, 5, 1-20.

15. Schneider, S.; Wright, C.M.; Heuckeroth, R.O. Unexpected Roles for the Second Brain: Enteric Nervous System as Master Regulator of Bowel Function. Annu. Rev. Physiol. 2019, 81, 235-259, doi:10.1146/annurev-physiol-021317121515.

16. Gershon, M.D. The enteric nervous system: a second brain. Hosp. Pract. (1995) 1999, 34, 31-32, 35-38, 41-32 passim.

17. Jandhyala, S.M.; Talukdar, R.; Subramanyam, C.; Vuyyuru, H.; Sasikala, M.; Nageshwar Reddy, D. Role of the normal gut microbiota. World J. Gastroenterol. 2015, 21, 8787-8803, doi:10.3748/wjg.v21.i29.8787.

18. Frank, D.N.; St Amand, A.L.; Feldman, R.A.; Boedeker, E.C.; Harpaz, N.; Pace, N.R. Molecular-phylogenetic characterization of microbial community imbalances in human inflammatory bowel diseases. Proc. Natl. Acad. Sci. U. S. A. 2007, 104, 13780-13785, doi:10.1073/pnas.0706625104.

19. Haiser, H.J.; Turnbaugh, P.J. Developing a metagenomic view of xenobiotic metabolism. Pharmacol. Res. 2013, 69, 21-31, doi:10.1016/j.phrs.2012.07.009.

20. Kuethe, J.W.; Armocida, S.M.; Midura, E.F.; Rice, T.C.; Hildeman, D.A.; Healy, D.P.; Caldwell, C.C. Fecal Microbiota Transplant Restores Mucosal Integrity in a Murine Model of Burn Injury. Shock 2015, 10.1097/SHK.0000000000000551, doi:10.1097/SHK.0000000000000551.

21. Donaldson, G.P.; Lee, S.M.; Mazmanian, S.K. Gut biogeography of the bacterial microbiota. Nat. Rev. Microbiol. 2016, 14, 20-32, doi:10.1038/nrmicro3552.

22. Kamada, N.; Chen, G.Y.; Inohara, N.; Nunez, G. Control of pathogens and pathobionts by the gut microbiota. Nat. Immunol. 2013, 14, 685-690, doi:10.1038/ni.2608.

23. Guida, F.; Turco, F.; Iannotta, M.; De Gregorio, D.; Palumbo, I.; Sarnelli, G.; Furiano, A.; Napolitano, F.; Boccella, S.; Luongo, L., et al. Antibiotic-induced microbiota perturbation causes gut endocannabinoidome changes, hippocampal 
neuroglial reorganization and depression in mice. Brain Behav. Immun. 2018, 67, 230-245, doi:10.1016/j.bbi.2017.09.001.

24. Zheng, P.; Zeng, B.; Zhou, C.; Liu, M.; Fang, Z.; Xu, X.; Zeng, L.; Chen, J.; Fan, S.; Du, X., et al. Gut microbiome remodeling induces depressive-like behaviors through a pathway mediated by the host's metabolism. Mol. Psychiatry 2016, 10.1038/mp.2016.44, doi:10.1038/mp.2016.44.

25. Mi, G.L.; Zhao, L.; Qiao, D.D.; Kang, W.Q.; Tang, M.Q.; Xu, J.K. Effectiveness of Lactobacillus reuteri in infantile colic and colicky induced maternal depression: a prospective single blind randomized trial. Antonie Van Leeuwenhoek 2015, 107, 1547-1553, doi:10.1007/s10482-015-0448-9.

26. Ni, J.; Wu, G.D.; Albenberg, L.; Tomov, V.T. Gut microbiota and IBD: causation or correlation? Nat. Rev. Gastroenterol Hepatol. 2017, 14, 573-584, doi:10.1038/nrgastro.2017.88.

27. Zhang, M.; Sun, K.; Wu, Y.; Yang, Y.; Tso, P.; Wu, Z. Interactions between Intestinal Microbiota and Host Immune Response in Inflammatory Bowel Disease. Front. Immunol. 2017, 8, 942, doi:10.3389/fimmu.2017.00942.

28. Cryan, J.F.; Dinan, T.G. Mind-altering microorganisms: the impact of the gut microbiota on brain and behaviour. Nat. Rev. Neurosci. 2012, 13, 701-712, doi:10.1038/nrn3346.

29. Waclawikova, B.; El Aidy, S. Role of Microbiota and Tryptophan Metabolites in the Remote Effect of Intestinal Inflammation on Brain and Depression. Pharmaceuticals (Basel) 2018, 11, doi:10.3390/ph11030063.

30. Takahashi, K.; Nishida, A.; Fujimoto, T.; Fujii, M.; Shioya, M.; Imaeda, H.; Inatomi, O.; Bamba, S.; Sugimoto, M.; Andoh, A. Reduced Abundance of Butyrate-Producing Bacteria Species in the Fecal Microbial Community in Crohn's Disease. Digestion 2016, 93, 59-65, doi:10.1159/000441768.

31. Parada Venegas, D.; De la Fuente, M.K.; Landskron, G.; Gonzalez, M.J.; Quera, R.; Dijkstra, G.; Harmsen, H.J.M.; Faber, K.N.; Hermoso, M.A. Short Chain Fatty Acids (SCFAs)-Mediated Gut Epithelial and Immune Regulation and Its Relevance for Inflammatory Bowel Diseases. Front. Immunol. 2019, 10, 277, doi:10.3389/fimmu.2019.00277.

32. Park, J.H.; Kotani, T.; Konno, T.; Setiawan, J.; Kitamura, Y.; Imada, S.; Usui, Y.; Hatano, N.; Shinohara, M.; Saito, Y., et al. Promotion of Intestinal Epithelial Cell Turnover by Commensal Bacteria: Role of Short-Chain Fatty Acids. PLoS ONE 2016, 11, e0156334, doi:10.1371/journal.pone.0156334.

33. Zheng, L.; Kelly, C.J.; Battista, K.D.; Schaefer, R.; Lanis, J.M.; Alexeev, E.E.; Wang, R.X.; Onyiah, J.C.; Kominsky, D.J.; Colgan, S.P. Microbial-Derived Butyrate Promotes Epithelial Barrier Function through IL-10 Receptor-Dependent Repression of Claudin-2. J. Immunol. 2017, 199, 2976-2984, doi:10.4049/jimmunol.1700105.

34. Donohoe, D.R.; Garge, N.; Zhang, X.; Sun, W.; O'Connell, T.M.; Bunger, M.K.; Bultman, S.J. The microbiome and butyrate regulate energy metabolism and autophagy in the mammalian colon. Cell Metab. 2011, 13, 517-526, doi:10.1016/j.cmet.2011.02.018.

35. Macia, L.; Tan, J.; Vieira, A.T.; Leach, K.; Stanley, D.; Luong, S.; Maruya, M.; lan McKenzie, C.; Hijikata, A.; Wong, C., et al. Metabolite-sensing receptors GPR43 and GPR109A facilitate dietary fibre-induced gut homeostasis through regulation of the inflammasome. Nat. Commun. 2015, 6, 6734, doi:10.1038/ncomms7734.

36. Lydiard, R.B. The role of GABA in anxiety disorders. J. Clin. Psychiatry 2003, 64 Supp/ 3, 21-27.

37. Orholm, M.; Munkholm, P.; Langholz, E.; Nielsen, O.H.; Sorensen, T.I.; Binder, V. Familial occurrence of inflammatory bowel disease. N. Engl. J. Med. 1991, 324, 84-88, doi:10.1056/NEJM199101103240203.

38. Kostic, A.D.; Xavier, R.J.; Gevers, D. The microbiome in inflammatory bowel disease: current status and the future ahead. Gastroenterology 2014, 146, 1489-1499, doi:10.1053/j.gastro.2014.02.009.

39. Jostins, L.; Ripke, S.; Weersma, R.K.; Duerr, R.H.; McGovern, D.P.; Hui, K.Y.; Lee, J.C.; Schumm, L.P.; Sharma, Y.; Anderson, C.A., et al. Host-microbe interactions have shaped the genetic architecture of inflammatory bowel disease. Nature 2012, 491, 119-124, doi:10.1038/nature11582.

40. Liu, J.Z.; van Sommeren, S.; Huang, H.; Ng, S.C.; Alberts, R.; Takahashi, A.; Ripke, S.; Lee, J.C.; Jostins, L.; Shah, T., et al. Association analyses identify 38 susceptibility loci for inflammatory bowel disease and highlight shared genetic risk across populations. Nat. Genet. 2015, 47, 979-986, doi:10.1038/ng.3359.

41. Cleynen, I.; Vazeille, E.; Artieda, M.; Verspaget, H.W.; Szczypiorska, M.; Bringer, M.A.; Lakatos, P.L.; Seibold, F.; Parnell, K.; Weersma, R.K., et al. Genetic and microbial factors modulating the ubiquitin proteasome system in inflammatory bowel disease. Gut 2014, 63, 1265-1274, doi:10.1136/gutjnl-2012-303205.

42. Cleynen, I.; Boucher, G.; Jostins, L.; Schumm, L.P.; Zeissig, S.; Ahmad, T.; Andersen, V.; Andrews, J.M.; Annese, V.; Brand, S., et al. Inherited determinants of Crohn's disease and ulcerative colitis phenotypes: a genetic association study. Lancet 2016, 387, 156-167, doi:10.1016/S0140-6736(15)00465-1. 
43. Momozawa, Y.; Dmitrieva, J.; Theatre, E.; Deffontaine, V.; Rahmouni, S.; Charloteaux, B.; Crins, F.; Docampo, E.; Elansary, M.; Gori, A.S., et al. IBD risk loci are enriched in multigenic regulatory modules encompassing putative causative genes. Nat. Commun. 2018, 9, 2427, doi:10.1038/s41467-018-04365-8.

44. Barthel, C.; Spalinger, M.R.; Brunner, J.; Lang, S.; Fried, M.; Rogler, G.; Scharl, M. A distinct pattern of diseaseassociated single nucleotide polymorphisms in IBD risk genes in a family with Crohn's disease. Eur J. Gastroenterol. Hepatol. 2014, 26, 803-806, doi:10.1097/MEG.0000000000000083.

45. Spehlmann, M.E.; Begun, A.Z.; Burghardt, J.; Lepage, P.; Raedler, A.; Schreiber, S. Epidemiology of inflammatory bowel disease in a German twin cohort: results of a nationwide study. Inflamm. Bowel Dis. 2008, 14, 968-976, doi:10.1002/ibd.20380.

46. Halfvarson, J.; Bodin, L.; Tysk, C.; Lindberg, E.; Jarnerot, G. Inflammatory bowel disease in a Swedish twin cohort: a long-term follow-up of concordance and clinical characteristics. Gastroenterology 2003, 124, 1767-1773.

47. Ardesia, M.; Ferlazzo, G.; Fries, W. Vitamin D and inflammatory bowel disease. Biomed. Res. Int. 2015, 2015, 470805, doi:10.1155/2015/470805.

48. Bashir, M.; Prietl, B.; Tauschmann, M.; Mautner, S.I.; Kump, P.K.; Treiber, G.; Wurm, P.; Gorkiewicz, G.; Hogenauer, C.; Pieber, T.R. Effects of high doses of vitamin D3 on mucosa-associated gut microbiome vary between regions of the human gastrointestinal tract. Eur. J. Nutr. 2016, 55, 1479-1489, doi:10.1007/s00394-015-0966-2.

49. Lahiri, A.; Abraham, C. Activation of pattern recognition receptors up-regulates metallothioneins, thereby increasing intracellular accumulation of zinc, autophagy, and bacterial clearance by macrophages. Gastroenterology 2014, 147, 835-846, doi:10.1053/j.gastro.2014.06.024.

50. Rogler, G.; Vavricka, S. Anemia in inflammatory bowel disease: an under-estimated problem? Front. Med. (Lausanne) 2014, 1, 58, doi:10.3389/fmed.2014.00058.

51. Mahid, S.S.; Minor, K.S.; Soto, R.E.; Hornung, C.A.; Galandiuk, S. Smoking and inflammatory bowel disease: a metaanalysis. Mayo Clin. Proc. 2006, 81, 1462-1471, doi:10.4065/81.11.1462.

52. Richardson, C.E.; Morgan, J.M.; Jasani, B.; Green, J.T.; Rhodes, J.; Williams, G.T.; Lindstrom, J.; Wonnacott, S.; Peel, S.; Thomas, G.A. Effect of smoking and transdermal nicotine on colonic nicotinic acetylcholine receptors in ulcerative colitis. QJM 2003, 96, 57-65.

53. Khor, B.; Gardet, A.; Xavier, R.J. Genetics and pathogenesis of inflammatory bowel disease. Nature 2011, 474, 307317, doi:10.1038/nature10209.

54. Pituch-Zdanowska, A.; Banaszkiewicz, A.; Albrecht, P. The role of dietary fibre in inflammatory bowel disease. Prz Gastroenterol. 2015, 10, 135-141, doi:10.5114/pg.2015.52753.

55. Hildebrandt, M.A.; Hoffmann, C.; Sherrill-Mix, S.A.; Keilbaugh, S.A.; Hamady, M.; Chen, Y.Y.; Knight, R.; Ahima, R.S.; Bushman, F.; Wu, G.D. High-fat diet determines the composition of the murine gut microbiome independently of obesity. Gastroenterology 2009, 137, 1716-1724 e1711-1712, doi:10.1053/j.gastro.2009.08.042.

56. Parks, B.W.; Nam, E.; Org, E.; Kostem, E.; Norheim, F.; Hui, S.T.; Pan, C.; Civelek, M.; Rau, C.D.; Bennett, B.J., et al. Genetic control of obesity and gut microbiota composition in response to high-fat, high-sucrose diet in mice. Cell Metab 2013, 17, 141-152, doi:10.1016/j.cmet.2012.12.007.

57. Geerling, B.J.; Dagnelie, P.C.; Badart-Smook, A.; Russel, M.G.; Stockbrugger, R.W.; Brummer, R.J. Diet as a risk factor for the development of ulcerative colitis. Am. J. Gastroenterol. 2000, 95, 1008-1013, doi:10.1111/j.15720241.2000.01942.x.

58. Aldini, R.; Micucci, M.; Cevenini, M.; Fato, R.; Bergamini, C.; Nanni, C.; Cont, M.; Camborata, C.; Spinozzi, S.; Montagnani, M., et al. Antiinflammatory effect of phytosterols in experimental murine colitis model: prevention, induction, remission study. PLoS One 2014, 9, e108112, doi:10.1371/journal.pone.0108112.

59. Brolinson, P.G.; Elliott, D. Exercise and the immune system. Clin. Sports Med. 2007, 26, 311-319, doi:10.1016/j.csm.2007.04.011.

60. Saxena, A.; Fletcher, E.; Larsen, B.; Baliga, M.S.; Durstine, J.L.; Fayad, R. Effect of exercise on chemically-induced colitis in adiponectin deficient mice. J. Inflamm. 2012, 9, 30, doi:10.1186/1476-9255-9-30.

61. Khalili, H.; Ananthakrishnan, A.N.; Konijeti, G.G.; Liao, X.; Higuchi, L.M.; Fuchs, C.S.; Spiegelman, D.; Richter, J.M.; Korzenik, J.R.; Chan, A.T. Physical activity and risk of inflammatory bowel disease: prospective study from the Nurses' Health Study cohorts. BMJ 2013, 347, f6633, doi:10.1136/bmj.f6633.

62. Ng, S.C.; Tang, W.; Leong, R.W.; Chen, M.; Ko, Y.; Studd, C.; Niewiadomski, O.; Bell, S.; Kamm, M.A.; de Silva, H.J., et al. Environmental risk factors in inflammatory bowel disease: a population-based case-control study in Asia-Pacific. Gut 2015, 64, 1063-1071, doi:10.1136/gutjnl-2014-307410. 
63. Loudon, C.P.; Corroll, V.; Butcher, J.; Rawsthorne, P.; Bernstein, C.N. The effects of physical exercise on patients with Crohn's disease. Am. J. Gastroenterol. 1999, 94, 697-703, doi:10.1111/j.1572-0241.1999.00939.x.

64. Engels, M.; Cross, R.K.; Long, M.D. Exercise in patients with inflammatory bowel diseases: current perspectives. Clin. Exp. Gastroenterol. 2018, 11, 1-11, doi:10.2147/CEG.S120816.

65. Bloomfield, S.F.; Stanwell-Smith, R.; Crevel, R.W.; Pickup, J. Too clean, or not too clean: the hygiene hypothesis and home hygiene. Clin. Exp. Allergy 2006, 36, 402-425, doi:10.1111/j.1365-2222.2006.02463.x.

66. Ng, S.C.; Bernstein, C.N.; Vatn, M.H.; Lakatos, P.L.; Loftus, E.V., Jr.; Tysk, C.; O'Morain, C.; Moum, B.; Colombel, J.F.; Epidemiology, et al. Geographical variability and environmental risk factors in inflammatory bowel disease. Gut 2013, 62, 630-649, doi:10.1136/gutjnl-2012-303661.

67. Koloski, N.A.; Bret, L.; Radford-Smith, G. Hygiene hypothesis in inflammatory bowel disease: a critical review of the literature. World J. Gastroenterol. 2008, 14, 165-173.

68. Carpio, D.; Barreiro-de Acosta, M.; Echarri, A.; Pereira, S.; Castro, J.; Ferreiro, R.; Lorenzo, A.; Group, E. Influence of urban/rural and coastal/inland environment on the prevalence, phenotype, and clinical course of inflammatory bowel disease patients from northwest of Spain: a cross-sectional study. Eur J. Gastroenterol. Hepatol 2015, 27, 1030-1037, doi:10.1097/MEG.0000000000000395.

69. Piovani, D.; Danese, S.; Peyrin-Biroulet, L.; Nikolopoulos, G.K.; Lytras, T.; Bonovas, S. Environmental Risk Factors for Inflammatory Bowel Diseases: an Umbrella Review of Meta-analyses. Gastroenterology 2019, 10.1053/j.gastro.2019.04.016, doi:10.1053/j.gastro.2019.04.016.

70. Ungaro, R.; Bernstein, C.N.; Gearry, R.; Hviid, A.; Kolho, K.L.; Kronman, M.P.; Shaw, S.; Van Kruiningen, H.; Colombel, J.F.; Atreja, A. Antibiotics associated with increased risk of new-onset Crohn's disease but not ulcerative colitis: a meta-analysis. Am. J. Gastroenterol. 2014, 109, 1728-1738, doi:10.1038/ajg.2014.246.

71. Ortizo, R.; Lee, S.Y.; Nguyen, E.T.; Jamal, M.M.; Bechtold, M.M.; Nguyen, D.L. Exposure to oral contraceptives increases the risk for development of inflammatory bowel disease: a meta-analysis of case-controlled and cohort studies. Eur J. Gastroenterol. Hepatol. 2017, 29, 1064-1070, doi:10.1097/MEG.0000000000000915.

72. Felder, J.B.; Korelitz, B.I.; Rajapakse, R.; Schwarz, S.; Horatagis, A.P.; Gleim, G. Effects of nonsteroidal antiinflammatory drugs on inflammatory bowel disease: a case-control study. Am. J. Gastroenterol. 2000, 95, 1949-1954, doi:10.1111/j.1572-0241.2000.02262.x.

73. Singh, S.; Graff, L.A.; Bernstein, C.N. Do NSAIDs, antibiotics, infections, or stress trigger flares in IBD? Am. J. Gastroenterol. 2009, 104, 1298-1313; quiz 1314, doi:10.1038/ajg.2009.15.

74. Guslandi, M. Exacerbation of inflammatory bowel disease by nonsteroidal anti-inflammatory drugs and cyclooxygenase-2 inhibitors: fact or fiction? World J. Gastroenterol. 2006, 12, 1509-1510.

75. Ananthakrishnan, A.N.; Long, M.D.; Martin, C.F.; Sandler, R.S.; Kappelman, M.D. Sleep disturbance and risk of active disease in patients with Crohn's disease and ulcerative colitis. Clin. Gastroenterol. Hepatol. 2013, 11, 965-971, doi:10.1016/j.cgh.2013.01.021.

76. Born, J.; Lange, T.; Hansen, K.; Molle, M.; Fehm, H.L. Effects of sleep and circadian rhythm on human circulating immune cells. J. Immunol. 1997, 158, 4454-4464.

77. Mahlmann, L.; Gerber, M.; Furlano, R.I.; Legeret, C.; Kalak, N.; Holsboer-Trachsler, E.; Brand, S. Impaired objective and subjective sleep in children and adolescents with inflammatory bowel disease compared to healthy controls. Sleep Med. 2017, 39, 25-31, doi:10.1016/j.sleep.2017.08.015.

78. Keefer, L.; Stepanski, E.J.; Ranjbaran, Z.; Benson, L.M.; Keshavarzian, A. An initial report of sleep disturbance in inactive inflammatory bowel disease. J. Clin. Sleep Med. 2006, 2, 409-416.

79. Ali, T.; Madhoun, M.F.; Orr, W.C.; Rubin, D.T. Assessment of the relationship between quality of sleep and disease activity in inflammatory bowel disease patients. Inflamm. Bowel Dis. 2013, 19, 2440-2443, doi:10.1097/MIB.0b013e3182a0ea54.

80. Voigt, R.M.; Forsyth, C.B.; Green, S.J.; Mutlu, E.; Engen, P.; Vitaterna, M.H.; Turek, F.W.; Keshavarzian, A. Circadian disorganization alters intestinal microbiota. PLOS ONE 2014, 9, e97500, doi:10.1371/journal.pone.0097500.

81. Bharwani, A.; Mian, M.F.; Foster, J.A.; Surette, M.G.; Bienenstock, J.; Forsythe, P. Structural \& functional consequences of chronic psychosocial stress on the microbiome \& host. Psychoneuroendocrinology 2016, 63, 217227, doi:10.1016/j.psyneuen.2015.10.001.

82. Bengmark, S. Gut microbiota, immune development and function. Pharmacol Res. 2013, 69, 87-113, doi:10.1016/j.phrs.2012.09.002. 
83. Xie, R.; Sun, Y.; Wu, J.; Huang, S.; Jin, G.; Guo, Z.; Zhang, Y.; Liu, T.; Liu, X.; Cao, X., et al. Maternal High Fat Diet Alters Gut Microbiota of Offspring and Exacerbates DSS-Induced Colitis in Adulthood. Front. Immunol. 2018, 9, 2608, doi:10.3389/fimmu.2018.02608.

84. Golubeva, A.V.; Crampton, S.; Desbonnet, L.; Edge, D.; O'Sullivan, O.; Lomasney, K.W.; Zhdanov, A.V.; Crispie, F.; Moloney, R.D.; Borre, Y.E., et al. Prenatal stress-induced alterations in major physiological systems correlate with gut microbiota composition in adulthood. Psychoneuroendocrinology 2015, 60, 58-74, doi:10.1016/j.psyneuen.2015.06.002.

85. Jasarevic, E.; Howerton, C.L.; Howard, C.D.; Bale, T.L. Alterations in the Vaginal Microbiome by Maternal Stress Are Associated With Metabolic Reprogramming of the Offspring Gut and Brain. Endocrinology 2015, 156, 3265-3276, doi:10.1210/en.2015-1177.

86. Collado, M.C.; Cernada, M.; Bauerl, C.; Vento, M.; Perez-Martinez, G. Microbial ecology and host-microbiota interactions during early life stages. Gut Microbes 2012, 3, 352-365, doi:10.4161/gmic.21215.

87. Donnet-Hughes, A.; Perez, P.F.; Dore, J.; Leclerc, M.; Levenez, F.; Benyacoub, J.; Serrant, P.; Segura-Roggero, I.; Schiffrin, E.J. Potential role of the intestinal microbiota of the mother in neonatal immune education. Proc. Nutr Soc. 2010, 69, 407-415, doi:10.1017/S0029665110001898.

88. Bailey, M.T.; Dowd, S.E.; Galley, J.D.; Hufnagle, A.R.; Allen, R.G.; Lyte, M. Exposure to a social stressor alters the structure of the intestinal microbiota: implications for stressor-induced immunomodulation. Brain Behav. Immun. 2011, 25, 397-407, doi:10.1016/j.bbi.2010.10.023.

89. Marin, I.A.; Goertz, J.E.; Ren, T.; Rich, S.S.; Onengut-Gumuscu, S.; Farber, E.; Wu, M.; Overall, C.C.; Kipnis, J.; Gaultier, A. Microbiota alteration is associated with the development of stress-induced despair behavior. Sci. Rep. 2017, 7, 43859, doi:10.1038/srep43859.

90. Galley, J.D.; Nelson, M.C.; Yu, Z.; Dowd, S.E.; Walter, J.; Kumar, P.S.; Lyte, M.; Bailey, M.T. Exposure to a social stressor disrupts the community structure of the colonic mucosa-associated microbiota. BMC Microbiol. 2014, 14, 189, doi:10.1186/1471-2180-14-189.

91. Bailey, M.T.; Coe, C.L. Maternal separation disrupts the integrity of the intestinal microflora in infant rhesus monkeys. Dev. Psychobiol. 1999, 35, 146-155.

92. O'Mahony, S.M.; Marchesi, J.R.; Scully, P.; Codling, C.; Ceolho, A.M.; Quigley, E.M.; Cryan, J.F.; Dinan, T.G. Early life stress alters behavior, immunity, and microbiota in rats: implications for irritable bowel syndrome and psychiatric illnesses. Biol. Psychiatry 2009, 65, 263-267, doi:10.1016/j.biopsych.2008.06.026.

93. Gao, X.; Cao, Q.; Cheng, Y.; Zhao, D.; Wang, Z.; Yang, H.; Wu, Q.; You, L.; Wang, Y.; Lin, Y., et al. Chronic stress promotes colitis by disturbing the gut microbiota and triggering immune system response. Proc. Natl. Acad. Sci. U. S. A. 2018, 115, E2960-E2969, doi:10.1073/pnas.1720696115.

94. Soderholm, J.D.; Yang, P.C.; Ceponis, P.; Vohra, A.; Riddell, R.; Sherman, P.M.; Perdue, M.H. Chronic stress induces mast cell-dependent bacterial adherence and initiates mucosal inflammation in rat intestine. Gastroenterology 2002, 123, 1099-1108.

95. Saunders, P.R.; Santos, J.; Hanssen, N.P.; Yates, D.; Groot, J.A.; Perdue, M.H. Physical and psychological stress in rats enhances colonic epithelial permeability via peripheral CRH. Dig. Dis Sci 2002, 47, 208-215.

96. Meddings, J.B.; Swain, M.G. Environmental stress-induced gastrointestinal permeability is mediated by endogenous glucocorticoids in the rat. Gastroenterology 2000, 119, 1019-1028.

97. Santos, J.; Yang, P.C.; Soderholm, J.D.; Benjamin, M.; Perdue, M.H. Role of mast cells in chronic stress induced colonic epithelial barrier dysfunction in the rat. Gut 2001, 48, 630-636.

98. Gareau, M.G.; Sherman, P.M.; Walker, W.A. Probiotics and the gut microbiota in intestinal health and disease. Nat. Rev. Gastroenterol. Hepatol. 2010, 7, 503-514, doi:10.1038/nrgastro.2010.117.

99. Zareie, M.; Johnson-Henry, K.; Jury, J.; Yang, P.C.; Ngan, B.Y.; McKay, D.M.; Soderholm, J.D.; Perdue, M.H.; Sherman, P.M. Probiotics prevent bacterial translocation and improve intestinal barrier function in rats following chronic psychological stress. Gut 2006, 55, 1553-1560, doi:10.1136/gut.2005.080739.

100. Garcia-Rodenas, C.L.; Bergonzelli, G.E.; Nutten, S.; Schumann, A.; Cherbut, C.; Turini, M.; Ornstein, K.; Rochat, F.; Corthesy-Theulaz, I. Nutritional approach to restore impaired intestinal barrier function and growth after neonatal stress in rats. J. Pediatr. Gastroenterol. Nutr. 2006, 43, 16-24, doi:10.1097/01.mpg.0000226376.95623.9f.

101. Noguera, J.C.; Aira, M.; Perez-Losada, M.; Dominguez, J.; Velando, A. Glucocorticoids modulate gastrointestinal microbiome in a wild bird. R. Soc. Open Sci. 2018, 5, 171743, doi:10.1098/rsos.171743.

102. Da Silva, S.; Robbe-Masselot, C.; Ait-Belgnaoui, A.; Mancuso, A.; Mercade-Loubiere, M.; Salvador-Cartier, C.; Gillet, M.; Ferrier, L.; Loubiere, P.; Dague, E., et al. Stress disrupts intestinal mucus barrier in rats via mucin O-glycosylation 
shift: prevention by a probiotic treatment. Am. J. Physiol Gastrointest Liver Physiol. 2014, 307, G420-429, doi:10.1152/ajpgi.00290.2013.

103. Walker, L.S.; Garber, J.; Smith, C.A.; Van Slyke, D.A.; Claar, R.L. The relation of daily stressors to somatic and emotional symptoms in children with and without recurrent abdominal pain. J. Consult. Clin. Psychol. 2001, 69, 85-91.

104. Zijlmans, M.A.; Korpela, K.; Riksen-Walraven, J.M.; de Vos, W.M.; de Weerth, C. Maternal prenatal stress is associated with the infant intestinal microbiota. Psychoneuroendocrinology 2015, 53, 233-245, doi:10.1016/j.psyneuen.2015.01.006.

105. Maes, M.; Kubera, M.; Leunis, J.C.; Berk, M. Increased IgA and IgM responses against gut commensals in chronic depression: further evidence for increased bacterial translocation or leaky gut. J. Affect. Disord. 2012, 141, 55-62, doi:10.1016/j.jad.2012.02.023.

106. van der Zaag-Loonen, H.J.; Grootenhuis, M.A.; Last, B.F.; Derkx, H.H. Coping strategies and quality of life of adolescents with inflammatory bowel disease. Qual. Life Res. 2004, 13, 1011-1019, doi:10.1023/B:QURE.0000025598.89003.0c.

107. McEwen, B.S.; Gianaros, P.J. Central role of the brain in stress and adaptation: links to socioeconomic status, health, and disease. Ann. N Y Acad. Sci. 2010, 1186, 190-222, doi:10.1111/j.1749-6632.2009.05331.x.

108. Sudo, N.; Chida, Y.; Aiba, Y.; Sonoda, J.; Oyama, N.; Yu, X.N.; Kubo, C.; Koga, Y. Postnatal microbial colonization programs the hypothalamic-pituitary-adrenal system for stress response in mice. J. Physiol. 2004, 558, 263-275, doi:10.1113/jphysiol.2004.063388.

109. Neufeld, K.M.; Kang, N.; Bienenstock, J.; Foster, J.A. Reduced anxiety-like behavior and central neurochemical change in germ-free mice. Neurogastroenterol. Motil. 2011, 23, 255-264, e119, doi:10.1111/j.1365-2982.2010.01620.x.

110. Diaz Heijtz, R.; Wang, S.; Anuar, F.; Qian, Y.; Bjorkholm, B.; Samuelsson, A.; Hibberd, M.L.; Forssberg, H.; Pettersson, S. Normal gut microbiota modulates brain development and behavior. Proc. Natl. Acad. Sci. U. S. A. 2011, 108, 30473052, doi:10.1073/pnas.1010529108.

111. Bravo, J.A.; Forsythe, P.; Chew, M.V.; Escaravage, E.; Savignac, H.M.; Dinan, T.G.; Bienenstock, J.; Cryan, J.F. Ingestion of Lactobacillus strain regulates emotional behavior and central GABA receptor expression in a mouse via the vagus nerve. Proc. Natl. Acad. Sci. U. S. A. 2011, 108, 16050-16055, doi:10.1073/pnas.1102999108.

112. Messaoudi, M.; Lalonde, R.; Violle, N.; Javelot, H.; Desor, D.; Nejdi, A.; Bisson, J.F.; Rougeot, C.; Pichelin, M.; Cazaubiel, M., et al. Assessment of psychotropic-like properties of a probiotic formulation (Lactobacillus helveticus R0052 and Bifidobacterium longum R0175) in rats and human subjects. Br. J. Nutr. 2011, 105, 755-764, doi:10.1017/S0007114510004319.

113. Li, N.; Wang, Q.; Wang, Y.; Sun, A.; Lin, Y.; Jin, Y.; Li, X. Oral Probiotics Ameliorate the Behavioral Deficits Induced by Chronic Mild Stress in Mice via the Gut Microbiota-Inflammation Axis. Front. Behav. Neurosci. 2018, 12, 266, doi:10.3389/fnbeh.2018.00266.

114. Rao, S.; Srinivasjois, R.; Patole, S. Prebiotic supplementation in full-term neonates: a systematic review of randomized controlled trials. Arch. Pediatr. Adolesc. Med. 2009, 163, 755-764, doi:10.1001/archpediatrics.2009.94.

115. Dhabhar, F.S. Effects of stress on immune function: the good, the bad, and the beautiful. Immunol. Res. 2014, 58, 193-210, doi:10.1007/s12026-014-8517-0.

116. Viswanathan, K.; Dhabhar, F.S. Stress-induced enhancement of leukocyte trafficking into sites of surgery or immune activation. Proc. Natl. Acad. Sci. U. S. A. 2005, 102, 5808-5813, doi:10.1073/pnas.0501650102.

117. Dhabhar, F.S.; Miller, A.H.; McEwen, B.S.; Spencer, R.L. Stress-induced changes in blood leukocyte distribution. Role of adrenal steroid hormones. J. Immunol. 1996, 157, 1638-1644.

118. Viswanathan, K.; Daugherty, C.; Dhabhar, F.S. Stress as an endogenous adjuvant: augmentation of the immunization phase of cell-mediated immunity. Int. Immunol. 2005, 17, 1059-1069, doi:10.1093/intimm/dxh286.

119. Maes, M.; Christophe, A.; Bosmans, E.; Lin, A.; Neels, H. In humans, serum polyunsaturated fatty acid levels predict the response of proinflammatory cytokines to psychologic stress. Biol. Psychiatry 2000, 47, 910-920.

120. Larson, M.R.; Ader, R.; Moynihan, J.A. Heart rate, neuroendocrine, and immunological reactivity in response to an acute laboratory stressor. Psychosom Med. 2001, 63, 493-501.

121. Schmid-Ott, G.; Jaeger, B.; Meyer, S.; Stephan, E.; Kapp, A.; Werfel, T. Different expression of cytokine and membrane molecules by circulating lymphocytes on acute mental stress in patients with atopic dermatitis in comparison with healthy controls. J. Allergy Clin. Immunol. 2001, 108, 455-462, doi:10.1067/mai.2001.117800.

122. Alsulami, S.; Al Omar, Z.; Binnwejim, M.S.; Alhamdan, F.; Aldrees, A.; Al-Bawardi, A.; Alsohim, M.; Alhabeeb, M. Perception of academic stress among Health Science Preparatory Program students in two Saudi universities. Adv. Med. Educ. Pract. 2018, 9, 159-164, doi:10.2147/AMEP.S143151. 
123. Connor, T.J.; Brewer, C.; Kelly, J.P.; Harkin, A. Acute stress suppresses pro-inflammatory cytokines TNF-alpha and IL-1 beta independent of a catecholamine-driven increase in IL-10 production. J. Neuroimmunol. 2005, 159, 119-128, doi:10.1016/j.jneuroim.2004.10.016.

124. Elenkov, I.J. Glucocorticoids and the Th1/Th2 balance. Ann. N. Y. Acad. Sci. 2004, 1024, 138-146, doi:10.1196/annals.1321.010.

125. Frank, M.G.; Frank, J.L.W.; Hendricks, S.E.; Burke, W.J.; Johnson, D.R. Age at onset of major depressive disorder predicts reductions in NK cell number and activity. J. Affect. Disord. 2002, 71, 159-167, doi:Pii S0165-0327(01)003950.

126. Danner, M.; Kasl, S.V.; Abramson, J.L.; Vaccarino, V. Association between depression and elevated C-reactive protein. Psychosom. Med. 2003, 65, 347-356, doi:10.1097/01.Psy.0000041542.29808.01.

127. Chang, Y.M.; El-Zaatari, M.; Kao, J.Y. Does stress induce bowel dysfunction? Expert. Rev. Gastroenterol. Hepatol. 2014, 8, 583-585, doi:10.1586/17474124.2014.911659.

128. Vrakas, S.; Mountzouris, K.C.; Michalopoulos, G.; Karamanolis, G.; Papatheodoridis, G.; Tzathas, C.; Gazouli, M. Intestinal Bacteria Composition and Translocation of Bacteria in Inflammatory Bowel Disease. PLoS ONE 2017, 12, e0170034, doi:10.1371/journal.pone.0170034.

129. Slyepchenko, A.; Maes, M.; Jacka, F.N.; Kohler, C.A.; Barichello, T.; Mclntyre, R.S.; Berk, M.; Grande, I.; Foster, J.A.; Vieta, E.; et al. Gut Microbiota, Bacterial Translocation, and Interactions with Diet: Pathophysiological Links between Major Depressive Disorder and Non-Communicable Medical Comorbidities. Psychother Psychosom 2017, 86, 31-46, doi:10.1159/000448957.

130. Mizoguchi, A.; Takeuchi, T.; Himuro, H.; Okada, T.; Mizoguchi, E. Genetically engineered mouse models for studying inflammatory bowel disease. J. Pathol. 2016, 238, 205-219, doi:10.1002/path.4640.

131. Singh, B.; Read, S.; Asseman, C.; Malmstrom, V.; Mottet, C.; Stephens, L.A.; Stepankova, R.; Tlaskalova, H.; Powrie, F. Control of intestinal inflammation by regulatory T cells. Immunol. Rev. 2001, 182, 190-200.

132. Tlaskalova-Hogenova, H.; Tuckova, L.; Stepankova, R.; Hudcovic, T.; Palova-Jelinkova, L.; Kozakova, H.; Rossmann, P.; Sanchez, D.; Cinova, J.; Hrncir, T., et al. Involvement of innate immunity in the development of inflammatory and autoimmune diseases. Ann. N. Y. Acad. Sci. 2005, 1051, 787-798, doi:10.1196/annals.1361.122.

133. Rabizadeh, S.; Rhee, K.J.; Wu, S.; Huso, D.; Gan, C.M.; Golub, J.E.; Wu, X.; Zhang, M.; Sears, C.L. Enterotoxigenic bacteroides fragilis: a potential instigator of colitis. Inflamm. Bowel Dis. 2007, 13, 1475-1483, doi:10.1002/ibd.20265.

134. Mahida, Y.R.; Makh, S.; Hyde, S.; Gray, T.; Borriello, S.P. Effect of Clostridium difficile toxin A on human intestinal epithelial cells: induction of interleukin 8 production and apoptosis after cell detachment. Gut 1996, 38, 337-347, doi:10.1136/gut.38.3.337.

135. Brito, G.A.; Fujji, J.; Carneiro-Filho, B.A.; Lima, A.A.; Obrig, T.; Guerrant, R.L. Mechanism of Clostridium difficile toxin A-induced apoptosis in T84 cells. J. Infect. Dis. 2002, 186, 1438-1447, doi:10.1086/344729.

136. Ghia, J.E.; Blennerhassett, P.; Deng, Y.; Verdu, E.F.; Khan, W.I.; Collins, S.M. Reactivation of inflammatory bowel disease in a mouse model of depression. Gastroenterology 2009, 136, 2280-2288 e2281-2284, doi:10.1053/j.gastro.2009.02.069.

137. Zamani, S.; Hesam Shariati, S.; Zali, M.R.; Asadzadeh Aghdaei, H.; Sarabi Asiabar, A.; Bokaie, S.; Nomanpour, B.; Sechi, L.A.; Feizabadi, M.M. Detection of enterotoxigenic Bacteroides fragilis in patients with ulcerative colitis. Gut Pathog. 2017, 9, 53, doi:10.1186/s13099-017-0202-0.

138. Issa, M.; Vijayapal, A.; Graham, M.B.; Beaulieu, D.B.; Otterson, M.F.; Lundeen, S.; Skaros, S.; Weber, L.R.; Komorowski, R.A.; Knox, J.F., et al. Impact of Clostridium difficile on inflammatory bowel disease. Clin. Gastroenterol. Hepatol. 2007, 5, 345-351, doi:10.1016/j.cgh.2006.12.028.

139. Baumgart, M.; Dogan, B.; Rishniw, M.; Weitzman, G.; Bosworth, B.; Yantiss, R.; Orsi, R.H.; Wiedmann, M.; McDonough, P.; Kim, S.G., et al. Culture independent analysis of ileal mucosa reveals a selective increase in invasive Escherichia coli of novel phylogeny relative to depletion of Clostridiales in Crohn's disease involving the ileum. ISME J. 2007, 1, 403-418, doi:10.1038/ismej.2007.52.

140. Darfeuille-Michaud, A.; Boudeau, J.; Bulois, P.; Neut, C.; Glasser, A.L.; Barnich, N.; Bringer, M.A.; Swidsinski, A.; Beaugerie, L.; Colombel, J.F. High prevalence of adherent-invasive Escherichia coli associated with ileal mucosa in Crohn's disease. Gastroenterology 2004, 127, 412-421.

141. Ford, D.E.; Erlinger, T.P. Depression and C-reactive protein in US adults: data from the Third National Health and Nutrition Examination Survey. Arch. Intern. Med. 2004, 164, 1010-1014, doi:10.1001/archinte.164.9.1010. 
142. Tuglu, C.; Kara, S.H.; Caliyurt, O.; Vardar, E.; Abay, E. Increased serum tumor necrosis factor-alpha levels and treatment response in major depressive disorder. Psychopharmacology 2003, 170, 429-433, doi:10.1007/s00213003-1566-z.

143. de Punder, K.; Pruimboom, L. Stress induces endotoxemia and low-grade inflammation by increasing barrier permeability. Front. Immunol. 2015, 6, 223, doi:10.3389/fimmu.2015.00223.

144. Whitehead, W.E.; Palsson, O.; Jones, K.R. Systematic review of the comorbidity of irritable bowel syndrome with other disorders: what are the causes and implications? Gastroenterology 2002, 122, 1140-1156.

145. Loftus, E.V., Jr.; Guerin, A.; Yu, A.P.; Wu, E.Q.; Yang, M.; Chao, J.; Mulani, P.M. Increased risks of developing anxiety and depression in young patients with Crohn's disease. Am. J. Gastroenterol. 2011, 106, 1670-1677, doi:10.1038/ajg.2011.142.

146. Targownik, L.E.; Sexton, K.A.; Bernstein, M.T.; Beatie, B.; Sargent, M.; Walker, J.R.; Graff, L.A. The Relationship Among Perceived Stress, Symptoms, and Inflammation in Persons With Inflammatory Bowel Disease. Am. J. Gastroenterol. 2015, 110, 1001-1012; quiz 1013, doi:10.1038/ajg.2015.147.

147. Coskun, M.; Vermeire, S.; Nielsen, O.H. Novel Targeted Therapies for Inflammatory Bowel Disease. Trends Pharmacol. Sci. 2017, 38, 127-142, doi:10.1016/j.tips.2016.10.014.

148. Torres, J.; Ellul, P.; Langhorst, J.; Mikocka-Walus, A.; Barreiro-de Acosta, M.; Basnayake, C.; Ding, N.J.S.; Gilardi, D.; Katsanos, K.; Moser, G., et al. European Crohn's and Colitis Organisation Topical Review on Complementary Medicine and Psychotherapy in Inflammatory Bowel Disease. J. Crohns Colitis 2019, 13, 673-685e, doi:10.1093/ecco-jcc/jjz051.

149. Regueiro, M.; Greer, J.B.; Szigethy, E. Etiology and Treatment of Pain and Psychosocial Issues in Patients With Inflammatory Bowel Diseases. Gastroenterology 2017, 152, 430-439 e434, doi:10.1053/j.gastro.2016.10.036.

150. Baird, C.L.; Sands, L.P. Effect of guided imagery with relaxation on health-related quality of life in older women with osteoarthritis. Res. Nurs. Health 2006, 29, 442-451, doi:10.1002/nur.20159.

151. Garcia-Vega, E.; Fernandez-Rodriguez, C. A stress management programme for Crohn's disease. Behav. Res. Ther. 2004, 42, 367-383, doi:10.1016/S0005-7967(03)00146-3.

152. Keefer, L.; Kiebles, J.L.; Martinovich, Z.; Cohen, E.; Van Denburg, A.; Barrett, T.A. Behavioral interventions may prolong remission in patients with inflammatory bowel disease. Behav. Res. Ther. 2011, 49, 145-150, doi:10.1016/j.brat.2010.12.005.

153. McCombie, A.M.; Mulder, R.T.; Gearry, R.B. Psychotherapy for inflammatory bowel disease: a review and update. J. Crohns Colitis 2013, 7, 935-949, doi:10.1016/j.crohns.2013.02.004.

154. Vermeire, S.; Van Assche, G.; Rutgeerts, P. C-reactive protein as a marker for inflammatory bowel disease. Inflamm. Bowel Dis. 2004, 10, 661-665, doi:10.1097/00054725-200409000-00026.

155. Gerbarg, P.L.; Jacob, V.E.; Stevens, L.; Bosworth, B.P.; Chabouni, F.; DeFilippis, E.M.; Warren, R.; Trivellas, M.; Patel, P.V.; Webb, C.D., et al. The Effect of Breathing, Movement, and Meditation on Psychological and Physical Symptoms and Inflammatory Biomarkers in Inflammatory Bowel Disease: A Randomized Controlled Trial. Inflamm. Bowel Dis. 2015, 21, 2886-2896, doi:10.1097/MIB.0000000000000568.

156. Neilson, K.; Ftanou, M.; Monshat, K.; Salzberg, M.; Bell, S.; Kamm, M.A.; Connell, W.; Knowles, S.R.; Sevar, K.; Mancuso, S.G., et al. A Controlled Study of a Group Mindfulness Intervention for Individuals Living With Inflammatory Bowel Disease. Inflamm. Bowel Dis. 2016, 22, 694-701, doi:10.1097/MIB.0000000000000629.

157. Langhorst, J.; Mueller, T.; Luedtke, R.; Franken, U.; Paul, A.; Michalsen, A.; Schedlowski, M.; Dobos, G.J.; Elsenbruch, S. Effects of a comprehensive lifestyle modification program on quality-of-life in patients with ulcerative colitis: a twelve-month follow-up. Scand. J. Gastroenterol. 2007, 42, 734-745, doi:10.1080/00365520601101682.

158. Szigethy, E.; Kenney, E.; Carpenter, J.; Hardy, D.M.; Fairclough, D.; Bousvaros, A.; Keljo, D.; Weisz, J.; Beardslee, W.R.; Noll, R., et al. Cognitive-behavioral therapy for adolescents with inflammatory bowel disease and subsyndromal depression. J. Am. Acad. Child. Adolesc Psychiatry 2007, 46, 1290-1298, doi:10.1097/chi.0b013e3180f6341f.

159. Keefer, L.; Kiebles, J.L.; Kwiatek, M.A.; Palsson, O.; Taft, T.H.; Martinovich, Z.; Barrett, T.A. The potential role of a selfmanagement intervention for ulcerative colitis: a brief report from the ulcerative colitis hypnotherapy trial. Biol. Res. Nurs. 2012, 14, 71-77, doi:10.1177/1099800410397629.

160. Larsson, K.; Sundberg Hjelm, M.; Karlbom, U.; Nordin, K.; Anderberg, U.M.; Loof, L. A group-based patient education programme for high-anxiety patients with Crohn disease or ulcerative colitis. Scand. J. Gastroenterol. 2003, 38, 763769.

161. Smith, G.D.; Watson, R.; Roger, D.; McRorie, E.; Hurst, N.; Luman, W.; Palmer, K.R. Impact of a nurse-led counselling service on quality of life in patients with inflammatory bowel disease. J. Adv. Nurs. 2002, 38, 152-160. 
162. Maunder, R.G.; Esplen, M.J. Supportive-expressive group psychotherapy for persons with inflammatory bowel disease. Can. J. Psychiatry 2001, 46, 622-626, doi:10.1177/070674370104600706.

163. Mizrahi, M.C.; Reicher-Atir, R.; Levy, S.; Haramati, S.; Wengrower, D.; Israeli, E.; Goldin, E. Effects of guided imagery with relaxation training on anxiety and quality of life among patients with inflammatory bowel disease. Psychol Health 2012, 27, 1463-1479, doi:10.1080/08870446.2012.691169.

164. Berrill, J.W.; Sadlier, M.; Hood, K.; Green, J.T. Mindfulness-based therapy for inflammatory bowel disease patients with functional abdominal symptoms or high perceived stress levels. J. Crohns Colitis 2014, 8, 945-955, doi:10.1016/j.crohns.2014.01.018.

165. Perera, L.P.; Radigan, M.; Guilday, C.; Banerjee, I.; Eastwood, D.; Babygirija, R.; Massey, B.T. Presence of Irritable Bowel Syndrome Symptoms in Quiescent Inflammatory Bowel Disease Is Associated with High Rate of Anxiety and Depression. Dig. Dis. Sci. 2019, 10.1007/s10620-019-05488-8, doi:10.1007/s10620-019-05488-8.

166. Gracie, D.J.; Irvine, A.J.; Sood, R.; Mikocka-Walus, A.; Hamlin, P.J.; Ford, A.C. Effect of psychological therapy on disease activity, psychological comorbidity, and quality of life in inflammatory bowel disease: a systematic review and metaanalysis. Lancet Gastroenterol. Hepatol. 2017, 2, 189-199, doi:10.1016/S2468-1253(16)30206-0.

167. Elsenbruch, S.; Langhorst, J.; Popkirowa, K.; Muller, T.; Luedtke, R.; Franken, U.; Paul, A.; Spahn, G.; Michalsen, A.; Janssen, O.E., et al. Effects of mind-body therapy on quality of life and neuroendocrine and cellular immune functions in patients with ulcerative colitis. Psychother. Psychosom. 2005, 74, 277-287, doi:10.1159/000086318.

168. Cheifetz, A.S.; Gianotti, R.; Luber, R.; Gibson, P.R. Complementary and Alternative Medicines Used by Patients With Inflammatory Bowel Diseases. Gastroenterology 2017, 152, 415-429 e415, doi:10.1053/j.gastro.2016.10.004.

169. Kimball, E.S.; Schneider, C.R.; Wallace, N.H.; Hornby, P.J. Agonists of cannabinoid receptor 1 and 2 inhibit experimental colitis induced by oil of mustard and by dextran sulfate sodium. Am. J. Physiol. Gastrointest Liver Physiol. 2006, 291, G364-371, doi:10.1152/ajpgi.00407.2005.

170. Massa, F.; Marsicano, G.; Hermann, H.; Cannich, A.; Monory, K.; Cravatt, B.F.; Ferri, G.L.; Sibaev, A.; Storr, M.; Lutz, B. The endogenous cannabinoid system protects against colonic inflammation. J. Clin. Invest. 2004, 113, 1202-1209, doi:10.1172/JCl19465.

171. Naftali, T.; Lev, L.B.; Yablecovitch, D.; Half, E.; Konikoff, F.M. Treatment of Crohn's disease with cannabis: an observational study. Isr Med. Assoc. J. 2011, 13, 455-458.

172. Naftali, T.; Bar-Lev Schleider, L.; Dotan, I.; Lansky, E.P.; Sklerovsky Benjaminov, F.; Konikoff, F.M. Cannabis induces a clinical response in patients with Crohn's disease: a prospective placebo-controlled study. Clin. Gastroenterol. Hepatol. 2013, 11, 1276-1280 e1271, doi:10.1016/j.cgh.2013.04.034.

173. Froicu, M.; Weaver, V.; Wynn, T.A.; McDowell, M.A.; Welsh, J.E.; Cantorna, M.T. A crucial role for the vitamin D receptor in experimental inflammatory bowel diseases. Mol. Endocrinol. 2003, 17, 2386-2392, doi:10.1210/me.20030281.

174. Jorgensen, S.P.; Agnholt, J.; Glerup, H.; Lyhne, S.; Villadsen, G.E.; Hvas, C.L.; Bartels, L.E.; Kelsen, J.; Christensen, L.A.; Dahlerup, J.F. Clinical trial: vitamin D3 treatment in Crohn's disease - a randomized double-blind placebo-controlled study. Aliment. Pharmacol Ther. 2010, 32, 377-383, doi:10.1111/j.1365-2036.2010.04355.x.

175. Miheller, P.; Muzes, G.; Hritz, I.; Lakatos, G.; Pregun, I.; Lakatos, P.L.; Herszenyi, L.; Tulassay, Z. Comparison of the effects of 1,25 dihydroxyvitamin $D$ and 25 hydroxyvitamin $D$ on bone pathology and disease activity in Crohn's disease patients. Inflamm. Bowel Dis. 2009, 15, 1656-1662, doi:10.1002/ibd.20947.

176. Ananthakrishnan, A.N. Vitamin D and Inflammatory Bowel Disease. Gastroenterol. Hepatol. 2016, 12, 513-515.

177. Pan, Y.; Liu, Y.; Guo, H.; Jabir, M.S.; Liu, X.; Cui, W.; Li, D. Associations between Folate and Vitamin B12 Levels and Inflammatory Bowel Disease: A Meta-Analysis. Nutrients 2017, 9, doi:10.3390/nu9040382.

178. O'Connor, E.M.; Grealy, G.; McCarthy, J.; Desmond, A.; Craig, O.; Shanahan, F.; Cashman, K.D. Effect of phylloquinone (vitamin K1) supplementation for 12 months on the indices of vitamin $\mathrm{K}$ status and bone health in adult patients with Crohn's disease. Br. J. Nutr. 2014, 112, 1163-1174, doi:10.1017/S0007114514001913.

179. Ng, V.; Millard, W.; Lebrun, C.; Howard, J. Low-intensity exercise improves quality of life in patients with Crohn's disease. Clin. J. Sport Med. 2007, 17, 384-388, doi:10.1097/JSM.0b013e31802b4fda.

180. Klare, P.; Nigg, J.; Nold, J.; Haller, B.; Krug, A.B.; Mair, S.; Thoeringer, C.K.; Christle, J.W.; Schmid, R.M.; Halle, M., et al. The impact of a ten-week physical exercise program on health-related quality of life in patients with inflammatory bowel disease: a prospective randomized controlled trial. Digestion 2015, 91, 239-247, doi:10.1159/000371795.

181. Sarkar, A.; Lehto, S.M.; Harty, S.; Dinan, T.G.; Cryan, J.F.; Burnet, P.W.J. Psychobiotics and the Manipulation of Bacteria-Gut-Brain Signals. Trends Neurosci 2016, 39, 763-781, doi:10.1016/j.tins.2016.09.002. 
Inflammatory Bowel Disease: A stressed "gut/feeling"

182. Akram, W.; Garud, N.; Joshi, R. Role of inulin as prebiotics on inflammatory bowel disease. Drug Discov. Ther. 2019, 13, 1-8, doi:10.5582/ddt.2019.01000. 



\section{Chapter 6}

\section{Pro-inflammatory implications of 2- hydroxypropyl- $\beta$-cyclodextrin treatment}

Tom Houben\#, Tulasi Yadati\#, Robbin de Kruijf, Marion JJ Gijbels, Joost J. F. P. Luiken, Marc van Zandvoort, Dimitris Kapsokalyvas, Dieter Lütjohann, Marit Westerterp, Jogchum Plat, David Leake, Ronit Shiri-Sverdlov

\#Authors equally contributed Frontiers in Immunology. 2021 August; 20; 12:716357. 


\begin{abstract}
Lifestyle- and genetically induced disorders related to disturbances in cholesterol metabolism have shown the detrimental impact of excessive cholesterol levels on a plethora of pathological processes such as inflammation. In this context, two- hydroxypropyl-b-cyclodextrin (CD) is increasingly considered as a novel pharmacological compound to decrease cellular cholesterol levels due to its ability to increase cholesterol solubility. However, recent findings have reported contra-indicating events after the use of $\mathrm{CD}$ questioning the clinical applicability of this compound. Given its potential as a therapeutic compound in metabolic inflammatory diseases, in this study, we evaluated the inflammatory effects of $C D$ administration in the context of cholesterol- induced metabolic inflammation in vivo and in vitro. The inflammatory and cholesterol- depleting effects of CD were first investigated in low-density lipoprotein receptor knockout (LdIr-/-) mice that were transplanted with $N p c 1^{\text {nih }}$ or $N p c 1^{\text {wt }}$ bone marrow and were fed either regular chow or a high-fat, high-cholesterol (HFC) diet for 12 weeks, thereby creating an extreme model of lysosomal cholesterol-induced metabolic inflammation. In the final three weeks, these mice received daily injections of either control (saline) or CD subcutaneously. Subsequently, the inflammatory properties of CD were investigated in vitro in two macrophage cell lines and in murine bone marrow-derived macrophages (BMDMs). While CD administration improved cholesterol mobilization outside lysosomes in BMDMs, an overall pro-inflammatory profile was observed after CD treatment, evidenced by increased hepatic inflammation in vivo and a strong increase in cytokine release and inflammatory gene expression in vitro in murine BMDMs and macrophages cell lines. Nevertheless, this CD-induced proinflammatory profile was time-dependent, as short-term exposure to $C D$ did not result in a proinflammatory response in BMDM. While CD exerts desired cholesterol-depleting effects, its inflammatory effect is dependent on the exposure time. As such, using CD in the clinic, especially in a metabolic inflammatory context, should be closely monitored as it may lead to undesired, proinflammatory side effects.
\end{abstract}




\section{Introduction}

As one of the most important steroid alcohols of the human body, cholesterol carries a number of essential functions ranging from acting as a key structural component of cell membranes [1] to serving as precursor for bile acids, steroid hormones and vitamin D metabolites [2]. Due to this key cellular function of cholesterol, a highly coordinated extra- and intracellular transport system maintains cholesterol homeostasis at a whole body as well as at a cellular level $[3,4]$. Evidently, deficiencies in this transport system leading to a disproportionate cellular supply of cholesterol result in severe pathogenic profiles. At one hand, these deficiencies can be induced via mutations in genes encoding for receptors and/or enzymes involved with cholesterol homeostasis. Examples of such disorders include Wolman disease [5] and the Niemann-Pick diseases [6] (both lysosomal storage disorders) as well as familial hypercholesterolemia [7]. Though these diseases are rather rare, their pathogenesis is often severe and sometimes results in premature death. Besides genetic predisposition, another and more prevalent manner that influences cholesterol homeostasis is via excessive overnutrition, leading to metabolic syndrome and its associated diseases [8]. In this context, excessive cholesterol accumulates at locations such as the vessel wall (atherosclerosis) and the liver (non-alcoholic fatty liver disease (NAFLD)) subsequently triggering resident macrophages to evolve into foam cells characterized by lysosomal cholesterol accumulation that induces an undesired inflammatory response, also referred to as metabolic inflammation [4, 9]. This inflammatory response is an antecedent for more severe consequences both in the context of atherosclerosis (heart attack, stroke, peripheral vascular disease [10]) as well as NAFLD (advanced liver diseases such as cirrhosis and hepatocellular carcinoma [9]). Taken the prevalence and the severity of these disorders into consideration, efficient therapeutic options aimed at reducing cellular cholesterol levels are needed.

A straightforward and successfully applied approach to reduce cholesterol levels is via pharmacological approaches, which include the use of statins [11], fibrates [12] and more recently antibodies targeting proprotein convertase subtilisin-kexin type 9 (PCSK9) [13]. However, substantial reduction of plasma cholesterol levels cannot be guaranteed for all patients [11], requiring alternative ways to improve cholesterol homeostasis. Relevantly, while the aforementioned interventions focus on reducing plasma cholesterol levels, a relative new approach aims at mobilizing cholesterol at the site of accumulation. This has led to the proposition to use 2-hydroxypropyl-bcyclodextrin (CD), a substance initially used to solubilize lipophilic pharmaceutical agents, as a compound to treat cholesterol accumulation. Indeed, CD mobilized cholesterol from foam cells [14], promotes atherosclerosis regression [15], improved hepatic cholesterol metabolism [16], reduced lysosomal size of neural stem cells derived from Wolman disease patients [17] and is under evaluation in advanced human clinical trials for Niemann-Pick disease, type C1 (NPC1) [18]. In contrast to these beneficial effects, CD causes massive damage to all cells of the developing [19] and some cells of the adult auditory system [20]. Moreover, while dietary supplementation of bcyclodextrin in hypercholesterolemic rats improved lipid metabolism, it concomitantly produced hepatotoxic effects characterized by increased plasma aminotransferase levels [21]. Together, though the use of $C D$ has clearly shown its benefits, the previous observations question whether this compound can be directly used in the clinic. Given its potential as therapeutic compound in metabolic inflammatory diseases, in this study, we therefore investigated the inflammatory aspects of $C D$ administration in the context of cholesterol-induced metabolic inflammation in vivo and in vitro. 
For this purpose, we investigated the inflammatory and cholesterol-depleting properties of $C D$ in vivo and in a series of in vitro experiments for metabolic inflammation. First, we analyzed the effect of $C D$ injection in low-density lipoprotein receptor knockout (Ldlr-/-) mice transplanted with $N p c 1^{\text {nih }}$ or $N p c 1^{\text {wt }}$ bone marrow on a high-fat, high-cholesterol (HFC) diet. Due to the absence of LDL receptors, and following an HFC diet, LdIr-/- mice are characterized by high plasma LDL levels, mimicking dietinduced dyslipidemia and serving as an excellent animal model for metabolic diseases. Furthermore, $N p c 1^{\text {nih }}$ bone marrow-transplanted mice feature a dysfunctional NPC1 protein in their immune cells, due to a deleterious frameshift mutation in the Npc1 allele, leading to lysosomal cholesterol buildup and a pro-inflammatory state. As such, chimeric Npc1nih transplanted (Npc1 ${ }^{\text {nih }}$-tp) Ldlr-/- mice given an HFC diet constitute an extreme state of lysosomal cholesterol-induced metabolic inflammation [22]. Next, as CD is able to mobilize lysosomal cholesterol, we assessed the ability of $C D$ to influence lysosomal size of metabolically challenged bone marrow-derived macrophages (BMDM) by employing confocal microscopy. Finally, in a series of in vitro experiments we investigated the inflammatory properties of $C D$ in more detail.

\section{Materials and methods}

\subsection{Mice, bone marrow transplant, diet and injections}

Throughout the study, mice were housed under standard conditions and had unlimited access to food and water, unless explicitly mentioned otherwise. For one week prior to and up to four weeks after bone marrow transplantation, $L d / r^{-/}$mice were housed in filter-top cages and received antibiotics diluted in drinking water to prevent infections following immunosuppression (Neomycin, $100 \mathrm{mg} / \mathrm{l}$, Gibco, Breda, the Netherlands; $6 * 10^{4} \mathrm{U} / \mathrm{L}$ polymycin B sulfate). Six weeks old bone marrow donors $N p c 1^{\text {nih }}$ and $N p c 1^{\text {wt }}$ mice were derived from heterozygous founders of a C57BL/6 genetic background. Genotype of $N p c 1^{\text {nih }}$ and $N p c 1^{\text {wt }}$ mice was determined as previously described [23]. On the day of the bone marrow transplant, $\mathrm{Npc}^{\text {nih }}$ and $\mathrm{Npc1}^{\text {wt }}$ littermates were sacrificed via $\mathrm{CO}_{2}$ inhalation and their bone marrows were isolated. One day before and on the day of the bone marrow transplant, $L d l r^{-/}$mice were subjected to six Gray of g-radiation, thus having received 12 Gray of gradiation before receiving $1^{*} 10^{7}$ bone marrow cells collected from $N p c 1^{\text {wt }}$ or $N p c 1^{\text {nih }}$ mice via intravenous injection. After ten weeks of recovery, transplanted mice were placed on a high-fat, highcholesterol (HFC) diet [24] for twelve weeks. In the final three weeks (based on [16, 25]), mice received daily, subcutaneous injections of control (saline) or 2-hydroxypropyl- b-cyclodextrin (1800 $\mathrm{mg} / \mathrm{kg}$ body weight; $\mathrm{CD}$, Sigma-Aldrich), creating four experimental groups: (1) Npc1 ${ }^{\text {wt }}$-tp $\mathrm{NaCl}(\mathrm{n}=$

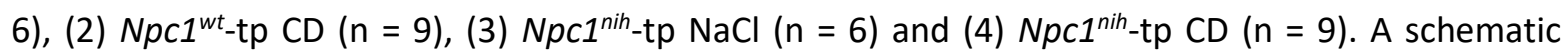
overview of the experimental set-up is provided in Supplementary Figure S1. All experiments were performed according to Dutch laws and approved by the Animal Experiment Committee of Maastricht University.

Upon sacrifice, all tissues were isolated and snap-frozen in liquid nitrogen and stored at $-80^{\circ} \mathrm{C}$ or fixed in $4 \%$ formaldehyde/ PBS. The collection of blood and tissue specimens, biochemical determination of lipids in plasma, RNA isolation, CDNA synthesis and qPCR were determined as described previously $[26,27]$. Primer sequences for genes are listed in Supplementary Table 1. Hepatic sterol content was determined by gas-liquid chromatography-mass spectroscopy, as described elsewhere [28]. 


\subsection{Immunohistochemistry}

Frozen liver sections $(7 \mu \mathrm{m})$ were fixed in acetone and blocked for endogenous peroxidase by incubation with $0.25 \%$ of $0.03 \% \mathrm{H}_{2} \mathrm{O}_{2}$ for 5 minutes. Primary antibodies used were against hepatic macrophages (1:100 rat anti-mouse CD68, clone FA11), infiltrated macrophages and neutrophils (1:500 rat anti- mouse Mac-1 (M1/70)) and neutrophils (1:100 rat anti-mouse NIMP (Ly6G)). 3Amino-9-ethylcarbazole (Vector laboratories, CA, USA) was applied as color substrate and hematoxylin for nuclear counterstain. Sections were enclosed with Faramount aqueous mounting medium. Additional information concerning the immunostainings is also described elsewhere [29].

Pictures were taken with a Nikon digital camera DMX1200 and ACT-1 v2.63 software (Nikon Instruments Europe, Amstelveen, The Netherlands). Infiltrated macrophages and neutrophil cells $\left(\mathrm{Mac}^{-1} \mathrm{1}^{+}\right.$) and neutrophils (NIMP) were counted by two blinded researchers in six microscopical views (original magnification, 200x) and were indicated as number of cells per square millimeter (cells $/ \mathrm{mm}^{2}$ ). Hepatic macrophages (CD68) were counted in six microscopical views (original magnification, 200x) and indicated as the percentage of CD68 positive area (Adobe Photoshop CS2 v.9.0.).

\subsection{Fluorescence confocal microscopy and quantification of lysosomes categorized by size}

\section{LAMP1- staining}

For assessing lysosomal size, we performed fluorescence confocal microscopy by staining for the lysosomal membrane marker lysosomal-associated membrane protein 1 (LAMP-1) followed by quantification of the number of lysosomes based on size. For this staining, fresh BMDM were fixed in paraformaldehyde (4\%) and permeabilized in Triton-X (0.1\%)/BSA (0.2\%) solution. BMDM were incubated with the primary (1:100, rabbit polyclonal Lamp1, ab24170, Abcam, Cambridge, United Kingdom) and secondary (1:200, Alexa fluor 488 goat anti-rabbit IgG, A11008, Thermo Fisher Scientific, Waltham, Massachusetts, USA) antibodies and finally, sections were enclosed with glycerol mounting medium (DABCO-DAPI). Confocal pictures were taken with a LEICA DMI 4000 microscope (Leica microsystems, Wetzlar, Germany), providing 3D images of BMDM and their lysosomes.

\section{Lysosomal quantification}

\section{Step 1: Configuring and adapting the lysosomal pictures}

For optimal quantification, one cell remained visible per purview. For this purpose, the original photo was cropped, and then configured into a TIFF file for further processing.

Step 2: Splitting the channels for nucleus and lysosomal quantification

In this study we made use of two fluorescent stainings either for the nucleus (DAPI) or lysosome (LAMP-1). To determine the nucleus to lysosomal distance, the color channels were bifurcated for better differentiation, creating a nucleus channel and lysosomal channel.

Step 3: Coordinates of the nucleus

To determine the lysosome to nucleus distance we first determined the location of the nucleus in the 3D picture. Therefore, we analyzed the nucleus channel with the 3D ObjectCounter.

Step 4: Creating a mask of lysosome channel 
To obtain the coordinates of the lysosomes, a mask was created by using the ObjectCounter. The mask was created by calculating the geometrical centers of the lysosomes [30]. Threshold was determined before the picture was analyzed.

\section{Step 5: Watershed}

To discriminate between separated or combined lysosomes, the watershed method was used. The watershed method uses the mask to determine the centers of the masses (Parra et al.).

Step 6: Coordinates of the lysosomes

After the watershed, the Regions of interest (ROIs) were added to the 3D ROI Manager, where the volume and coordinates were calculated. To quantify the pictures in an efficient manner, a macro was created (Supplementary methods).

\section{Step 7: Calculating the distance}

After the coordinates and volumes were obtained, the distance for each lysosome to the nucleus was calculated. For this we used the Pythagoras equation.

Distance $=V\left(\left(X_{\text {nucleus }}-X_{\text {ysosome }}\right)^{2}+\left(Y_{\text {nucleus }}-Y_{\text {lysosome }}\right)^{2}+\left(Z_{\text {nucleus }}-Z_{\text {lysosome }}\right)^{2}\right)$

Next, we created a plugin-Macro for ImageJ to identify the amount and volume of lysosomes. Finally, the number of lysosomes was quantified and categorized them by size $\left(<0.1 \mu \mathrm{m}^{3} ; 0.1-1 \mu \mathrm{m}^{3} ;>1 \mu \mathrm{m}^{3}\right)$ and expressed the number of lysosomes per size relative to the total amount of lysosomes present inside the BMDM. Representative videos were created using Z-stack images played in series and recorded as.AVI files which were trimmed using quick time movie player. DAPI (blue) represents nuclei and LAMP-1 (green) represents lysosomes.

\subsection{Statistical analysis}

Data were statistically analyzed by performing the unpaired t-test or the two-way ANOVA and Tukey's post hoc test using GraphPad Prism software (version 6 for Windows, GraphPad Software Inc, San Diego, CA, U.S; www.graphpad.com). Data were expressed as the group mean and standard error of the mean. 


\section{Results}

\subsection{Administration of 2-hydroxypropyl- $\beta$-cyclodextrin mobilizes hepatic cholesterol in Npc1 ${ }^{\text {nih }}$-tp Ldlr-/- mice}

$N p c 1^{\text {nih }}$ bone marrow-transplanted mice feature a dysfunctional NPC1 protein in their immune cells, due to a deleterious frameshift mutation in the Npc1 allele, leading to lysosomal cholesterol buildup and a pro-inflammatory state. As such, chimeric $N p c 1^{\text {nih }}$ transplanted ( $N p c 1^{\text {nih }}$-tp) $L d l r^{-/}$mice given an HFC diet constitute an extreme state of lysosomal cholesterol- induced metabolic inflammation [22].

To confirm successful injection of 2-hydroxypropyl-b-cyclodextrin $(C D)$ into the mice in the final three weeks of the experiments, plasma and hepatic lipid metabolism were profiled. CD-treated $N p c 1^{w t}$-tp LdIr $^{-1-}$ mice showed reduced plasma triglycerides (Table 1 ), though this reduction was already apparent before the start of $C D$ treatment (Table 1). While no effects were observed on plasma (Table 1) and hepatic (Figure 1) cholesterol levels, the ratios of the cholesterol oxidation products $7 a-$ hydroxycholesterol to cholesterol and 27-hydroxycholesterol to cholesterol were both significantly increased in the livers of CD-treated $N p c 1^{\text {nih }}$ - and $N p c 1^{\text {wt }}$-tp $L d l r^{-/}$mice, suggesting increased mobilization of cholesterol (Figures 1B, C). Hepatic gene expression analysis of cytochrome P450 7A1 (Cyp7a1), the enzyme responsible for the conversion of cholesterol into 7a-hydroxycholesterol, was concomitantly increased (Figure 1D), while sterol 27-hydroxylase (Cyp27a1) hepatic gene expression levels remained unaffected upon $C D$-treatment (Figure $1 \mathrm{E}$ ). Concerning other non-cholesterol sterols, CD-treatment reduced hepatic cholestanol and desmosterol levels in CD- treated Npc1 ${ }^{\text {nih }}$-tp $\mathrm{Ldll}^{-/}$mice (Table 2). No effects were observed on liver and spleen weight (Supplementary Figures

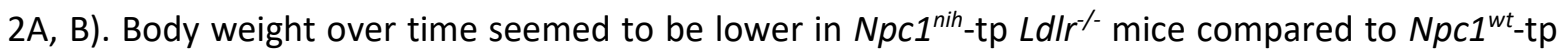
LdIr-- mice, but this difference was not significant (Supplementary Figure $2 \mathrm{C}$ ). Overall, these findings indicate that CD-treatment mobilizes hepatic cholesterol, confirming successful administration of CD.

A

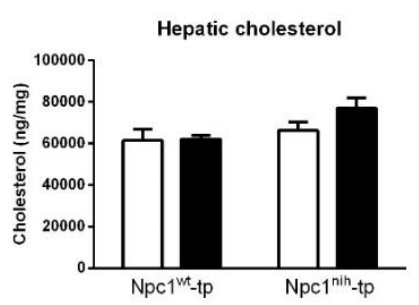

D

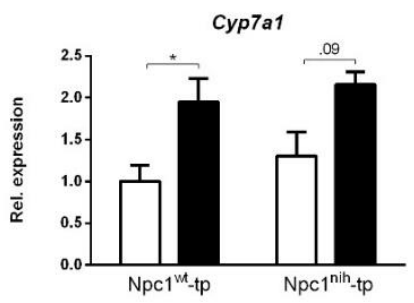

B

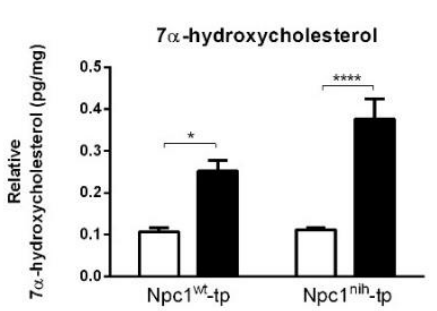

E

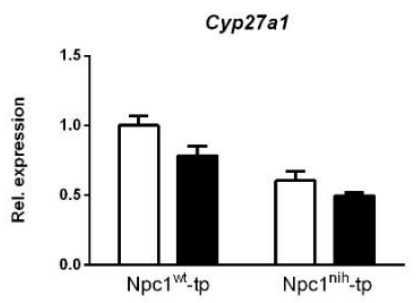

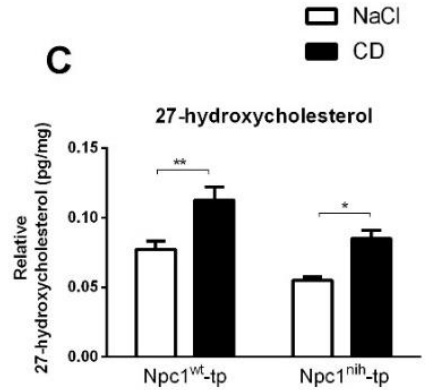

Figure 1 Hepatic lipid parameters. (A) Hepatic cholesterol levels of $N p c 1^{\text {wt }}$-tp and $N p c 1^{\text {nih }}$-tp mice on a HFC diet that received CD or saline treatment. (B-C) Hepatic levels of the cholesterol degradation products $7 \alpha$-hydroxycholesterol and 27-hydroxycholesterol. (D-E) Hepatic gene expression 
analysis of Cyp7a1 (D) and Cyp27a1 (E). $n=6-9$ mice/group. Gene expression data are set relative to $N p c 1^{w t}$-tp mice treated with saline. ${ }^{*}, * *$ and $* * * *$ indicate $p \leq 0.05,0.01$ and 0.0001 resp.by means of two-way ANOVA followed by Tukey post-hoc analysis.

Table 1: Plasma total cholesterol and triglyceride levels

\begin{tabular}{|c|c|c|c|c|}
\hline & \multicolumn{2}{|c|}{$N p c 1^{w t}$-tp } & \multicolumn{2}{|c|}{$N p c 1^{\text {nih_tp }}$} \\
\hline & $\mathrm{NaCl}$ & Cyclo & $\mathrm{NaCl}$ & Cyclo \\
\hline \multicolumn{5}{|c|}{ Plasma cholesterol (mM) } \\
\hline то & $9.48( \pm 0.86)$ & $7.87( \pm 0.73)$ & $6.372( \pm 0.30)$ & $5.81( \pm 0.69)$ \\
\hline T9 & $148.15( \pm 5.33)$ & $118.3( \pm 11.77)$ & $66.68( \pm 8.60)$ & $80.72( \pm 4.13)$ \\
\hline $\mathrm{T} 12$ & $137.15( \pm 4.73)$ & $120.62( \pm 9.68)$ & $97.97( \pm 6.61)$ & $112.76( \pm 8.48)$ \\
\hline \multicolumn{5}{|c|}{ Plasma triglycerides (mM) } \\
\hline T0 & $1.30( \pm 0.11)$ & $1.47( \pm 0.17)$ & $0.96( \pm 0.11)$ & $0.93( \pm 0.11)$ \\
\hline T9 & $5.57( \pm 0.82)$ & $2.99( \pm 0.44)^{* *}$ & $1.17( \pm 0.25)$ & $1.10( \pm 0.19)$ \\
\hline T12 & $3.18( \pm 0.47)$ & $1.88( \pm 0.46)^{* * *}$ & $0.58( \pm 0.13)$ & $0.64( \pm 0.11)$ \\
\hline
\end{tabular}

** and *** indicates $p<0.01$ and 0.001 by means of two-way ANOVA followed by Tukey post-hoc analysis.

Table 2: Hepatic non-cholesterol sterol concentrations

\begin{tabular}{|c|c|c|c|c|}
\hline & \multicolumn{2}{|c|}{$N p c 1^{w t}$-tp } & \multicolumn{2}{|c|}{$N p c 1^{\text {nih }}$-tp } \\
\hline & $\mathrm{NaCl}$ & Cyclo & $\mathrm{NaCl}$ & Cyclo \\
\hline \multicolumn{5}{|l|}{ Absorption markers } \\
\hline R_Sitosterol (pg/mg) & $0.05( \pm 0.01)$ & $0.05( \pm 0.01)$ & $0.13( \pm 0.01)$ & $0.12( \pm 0.01)$ \\
\hline R_Campestanol (pg/mg) & $0.12( \pm 0.01)$ & $0.12( \pm 0.01)$ & $0.14( \pm 0.03)$ & $0.12( \pm 0.02)$ \\
\hline R_Cholestanol (pg/mg) & $4.73( \pm 0.25)$ & $5.00( \pm 0.16)$ & $3.91( \pm 0.16)$ & $3.06( \pm 0.11)^{a}$ \\
\hline \multicolumn{5}{|l|}{ Cholesterol synthesis markers } \\
\hline R_Lathosterol (pg/mg) & $0.49( \pm 0.06)$ & $0.56( \pm 0.05)$ & $0.33( \pm 0.04)$ & $0.28( \pm 0.01)$ \\
\hline R_Desmosterol (pg/mg) & $0.29( \pm 0.02)$ & $0.30( \pm 0.01)$ & $1.17( \pm 0.10)$ & $0.84( \pm 0.09)^{b}$ \\
\hline R_Lanosterol (pg/mg) & $28.6( \pm 2.2)$ & $30.2( \pm 2.0)$ & $41.2( \pm 1.1)$ & $34.7( \pm 1.9)$ \\
\hline
\end{tabular}

Data is given as mean \pm standard error of the mean (S.E.M.).

$a, b$ indicates $p<0.05$ and $p<0.01$ respectively compared to $\mathrm{NaCl}$-injected $\mathrm{Npc}^{\text {nih }}$-tp mice.

All values are given as the ratio sterol to cholesterol.

\subsection{Administration of 2-hydroxypropyl- $\beta$-cyclodextrin increases hepatic inflammation and fibrotic markers in Npc1 ${ }^{\text {nih }}$-tp Ldlr-/- mice}

After we confirmed successful administration of $C D$, we further evaluated the impact of three-week CD treatment on hepatic inflammation by staining hepatic cryosections for the inflammatory markers Mac-1 (infiltrated macrophages and neutrophils; directed against Cd11b), NIMP (neutrophils) and CD68 (macrophages) (Figures 2A-C). While CD treatment only induced a minor non-significant increase for these inflammatory markers in $N p c 1^{\text {wt }}$-tp $L d l r^{-/}$mice, this increase was more pronounced in Npc1 $1^{\text {nih }}$-tp Ldlr/- mice (Figures 2A, D). This pro-inflammatory effect of CD was confirmed by histological scoring of HE-staining of livers of the $N p c 1^{\text {nih }}$-tp $L d / r^{-/}$mice (Supplementary Figure S3). To confirm these histological data, hepatic gene expression for the inflammatory markers tumor necrosis factor alpha (Tnfa), chemokine (C-C motif) ligand 2 (Cc/2), chemokine (C-C motif) ligand 5 (CCl5) and serum amyloid A1 (Saa1) was analyzed. In line with the histological data, CD treatment 
increased the expression of these hepatic inflammatory markers in the $N p c 1^{w t}$-tp or in the $N p c 1^{\text {nih }}$ tp group (Figures $2 \mathrm{E}-\mathrm{H}$ ). Moreover, the hepatic fibrotic markers matrix metallopeptidase 9 (Mmp9) and plasminogen activator inhibitor-1 (Pai-1) were concomitantly increased in CD-treated Npc1 ${ }^{\text {nih }}$-tp $L_{d l l} r^{-1}$ mice, while no significant changes were observed in transforming growth factor beta (Tgfb) and tissue inhibitor of metalloproteinase (Timp1) (Supplementary Figure S4). Together, these findings indicate that three-week treatment with $C D$ increased hepatic inflammation and mild elevations in fibrosis in our experimental model.

A

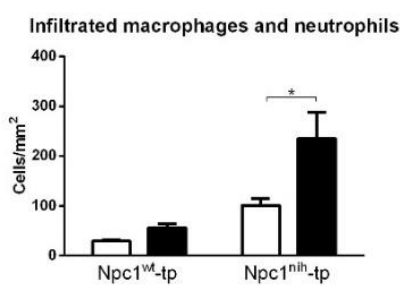

D

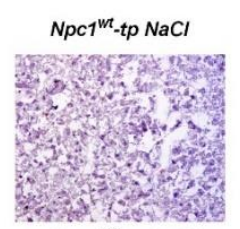

$\mathrm{Npc1}^{\text {nih }}$-tp $\mathrm{NaCl}$

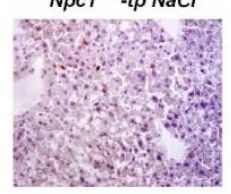

B

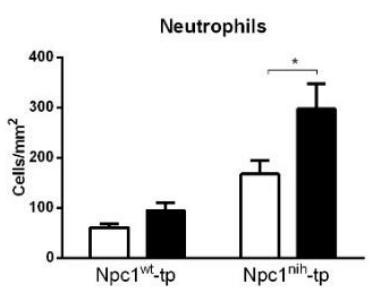

E

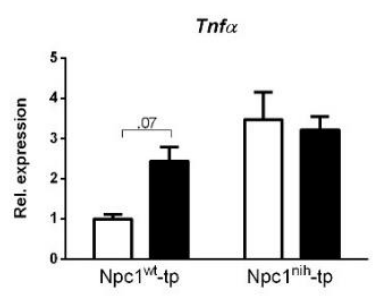

G

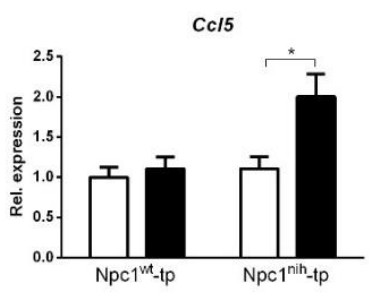

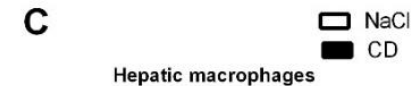

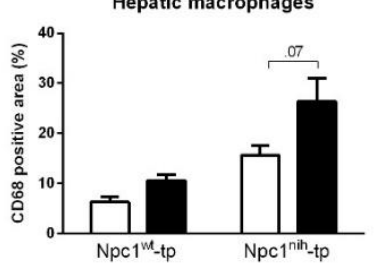

$\mathbf{F}$

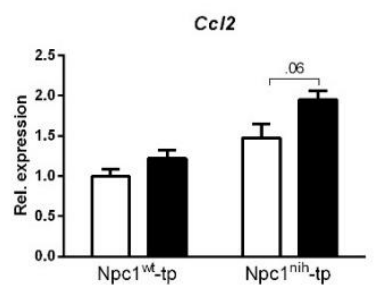

H

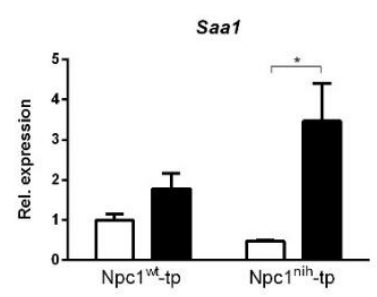

Figure 2 Parameters of hepatic inflammation: (A-C) Liver sections were stained for infiltrating macrophages and neutrophils (Mac-1), neutrophils (NIMP) and hepatic macrophages (CD68) and subsequently quantified by counting (Mac-1 and NIMP) or by assessing the positive area of the staining (CD68). (D) Representative images of liver sections stained for infiltrated macrophages and neutrophils (Mac-1). (E-H) Hepatic gene expression analysis of Tnfa, $C \mathrm{Cl} 2, \mathrm{CCl} 5$ and Saa1. Gene expression data were set relative to control-treated $N p c 1^{w t}$-tp mice. ${ }^{*}$ indicates $p \leq 0.05$ by means of two-way ANOVA followed by Tukey post-hoc analysis.

\subsection{Treatment with 2-hydroxypropyl- $\beta$-cyclodextrin normalizes lysosomal size of metabolically challenged bone marrow-derived macrophages}

As CD treatment mobilized cholesterol, but increased inflammation in our in vivo model for lysosomal cholesterol- induced inflammation, we opted to investigate the effect of $C D$ on lysosomal size in metabolically challenged BMDM via confocal microscopy. For this purpose, we first compared lysosomal size between wildtype and $N p c 1^{\text {nih }}$ BMDM. While $N p c 1^{\text {nih }}$ BMDM showed a reduced 
number of small lysosomes (categorized as $<0.1 \mu \mathrm{m}^{3}$ ), they showed an increase in the number of large lysosomes (categorized as $>1 \mu \mathrm{m}^{3}$ ) as compared to wildtype BMDM, which is likely the result of lysosomal lipid accumulation that is characteristic for the mutation (Figure 3 Comparison A; Supplementary Table 2).

As internalization of oxidized cholesterol-rich low-density lipoprotein (oxLDL) in BMDM also leads to lysosomal accumulation, we next compared lysosomal size of oxLDL $(25 \mu \mathrm{g} / \mathrm{ml} ; 72 \mathrm{hr})$-incubated wildtype BMDM to control- incubated wildtype BMDM. Incubating wildtype BMDM with oxLDL similarly reduced the number of small lysosomes and increased the number of large lysosomes, confirming the effect of oxLDL on lysosomal size (Figure 3 Comparison B; Supplementary Table 3) as well as our method to quantify the number of lysosomes based on their size. Representative videos of all indicated conditions are shown in Supplementary Videos 1-5. Next, to assess the impact of CD treatment, we compared lysosomal size of oxLDL-incubated wildtype BMDM that were treated with or without CD (1.95 mM; 4hr). As expected, CD treatment increased the number of small lysosomes and reduced the level of large lysosomes, thereby reversing the oxLDL- induced effects on lysosomal size (Figure 3 Comparison C; Supplementary Table 4). Furthermore, similar findings were observed when Npc1 ${ }^{\text {nih }}$ BMDM were treated with CD (Figure 3 Comparison D; Supplementary Table 5). Overall, these findings confirm the beneficial effect of $C D$ treatment on lysosomes of metabolically challenged BMDM.

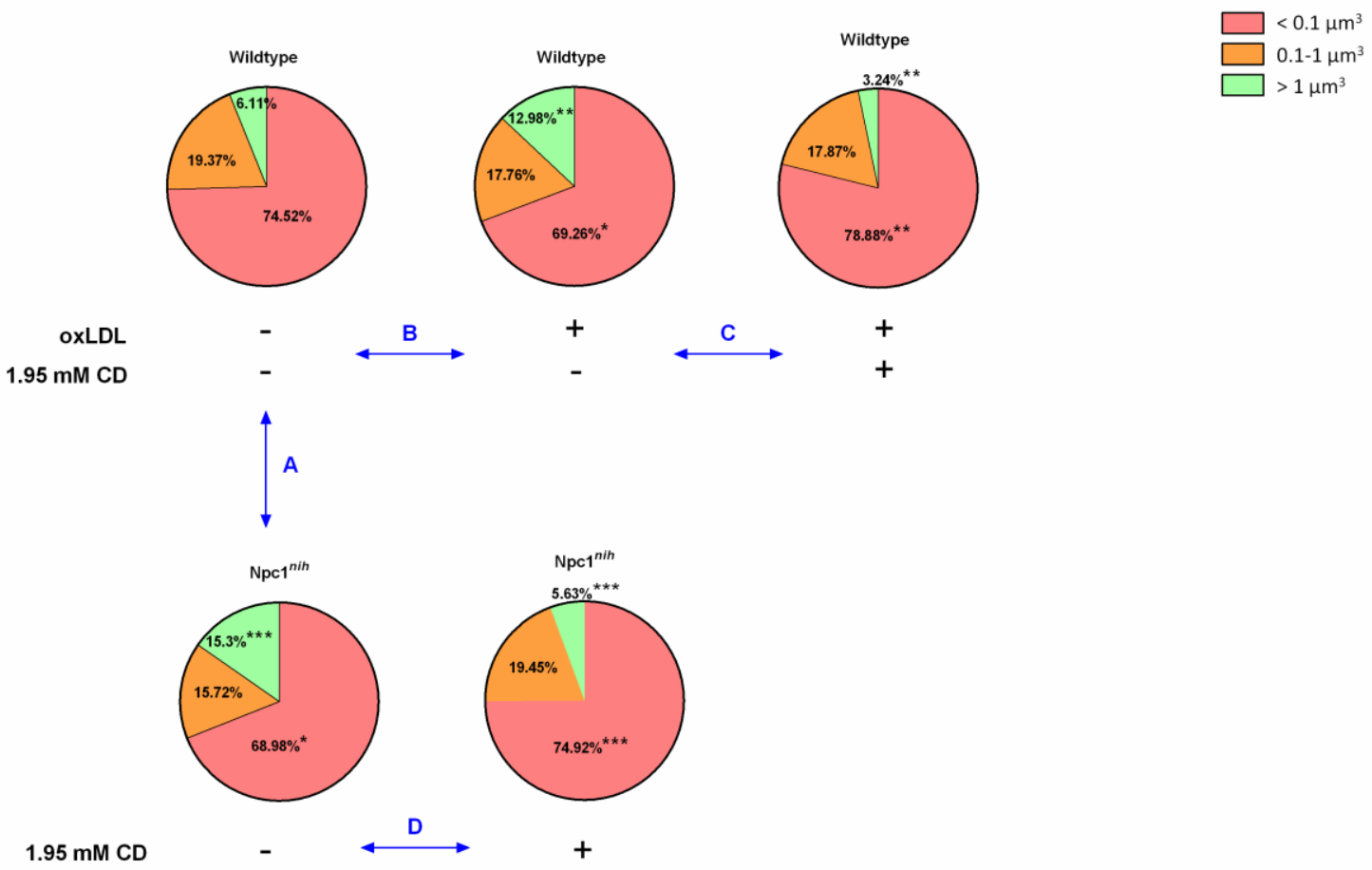

Figure 3 Lysosomal volume of metabolically-challenged bone marrow-derived macrophages with and without intervention: We quantified the number of lysosomes, categorized them by size $1<0.1$ $\mu \mathrm{m}^{3} ; 0.1-1 \mu \mathrm{m}^{3} ;>1 \mathrm{~m}^{3}$ ) and expressed the number of lysosomes per size relative to the total amount of lysosomes present inside the BMDM. Comparison A: Relative comparison of the distribution of lysosomal volumes between $W t$ and $N p c 1^{\text {nih }}$ BMDM. Comparison B: Relative comparison of the distribution of lysosomal volumes between control and oxLDL $(25 \mu \mathrm{g} / \mathrm{ml} ; 72 \mathrm{hr}$ ) -stimulated Wt BMDM. Comparison C: Relative comparison of the distribution of lysosomal 
volumes between oxLDL-stimulated Wt BMDM treated with or without CD $(1.95 \mathrm{mM}$; 4hr). Comparison D: Relative comparison of the distribution of lysosomal volumes between $N p c 1^{\text {ih }}$ BMDM treated with or without CD. ${ }^{*}, * *$ and ${ }^{* * *}$ indicate $p<0.05,0.01$ and 0.001 resp.by means of unpaired $t$ - test. Data are the result of analysis of $n=5-7$ BMDM per experimental condition.

\subsection{Incubation with 2-hydroxypropyl- $\beta$-cyclodextrin exerts pro-inflammatory effects in metabolically challenged bone marrow derived macrophages}

To increase our insight into the inflammatory effects of $C D$, we performed a series of in vitro experiments in BMDM, framed within the context of metabolic inflammation. First, we incubated wildtype and Npc1 ${ }^{\text {nih }}$ BMDM with CD for $4 \mathrm{hr}$ followed by $4 \mathrm{hr}$ lipopolysaccharide (LPS) stimulation. Four-hour CD treatment increased TNFa protein secretion in both wildtype and Npc1 ${ }^{\text {nih }}$ BMDM, suggesting an acute pro-inflammatory effect of $C D$ also in wildtype and $N p c 1^{\text {nih }} B M D M$ (Figure $4 A_{\text {; }}$ Supplementary Figure S5A). To provide stronger evidence for this inflammatory effect of CD, Npc1 ${ }^{\text {nih }}$ BMDM were challenged with oxLDL, followed by $4 \mathrm{hr} C D$ incubation and $4 \mathrm{hr}$ LPS stimulation, thereby creating a more severe model of metabolic inflammation. Similar to our previous findings, $C D$ treatment increased TNFa protein secretion in both oxLDL- and control-challenged $N p c 1^{\text {nih }}$ BMDM (Figure 4B; Supplementary Figure S5B). This was confirmed at gene expression level, showing increased levels of pro- inflammatory markers (Tnfa, iNos/Arg1 ratio) (Figure 4B; Supplementary Figure S5B) and decreased levels of anti- inflammatory markers (IL-10; Figure 4B; Supplementary Figure $\mathrm{S} 5 \mathrm{~B}$ ) after $\mathrm{CD}$ treatment. These pro-inflammatory findings of $C D$ were also confirmed in a separate BMDM experiment with similar set-up in wildtype BMDM (Supplementary Figure S6A) and without the final LPS stimulus (Supplementary Figure S6B).

As another model for metabolic disturbance, we investigated the inflammatory effects of CD on Ldlr I- BMDM, which is a mutation causing disturbances in cholesterol metabolism. Twenty-four-hour incubation with oxLDL followed by $4 \mathrm{hr} C D$ treatment resulted in increased gene expression levels of Tnfa, $I L-1 b$, and $I L-18$, confirming the pro-inflammatory effects of $C D$ also in $L d I r^{-1}$ BMDM (Figure $4 C$; Supplementary Figure S4C). Nevertheless, CD treatment increased Lxra and Npc2 expression, confirming the cholesterol-mobilizing properties of $\mathrm{CD}$. To further confirm these findings, a similar experiment was conducted with the addition of a final stimulation with Pam3Cys or LPS for $4 \mathrm{hr}$. Stimulation with Pam3Cys (Figure 4D; Supplementary Figure S4D) and LPS (Figure 4E; Supplementary Figure S4E) showed similar results as previously described, showing pro-inflammatory and cholesterol-mobilizing effects of $\mathrm{CD}$ treatment in $L d / r^{/-}$BMDM. Together, these findings show that while CD maintains its effect on cholesterol metabolism, pro-inflammatory effects become apparent after $4 \mathrm{hr}$ incubation in metabolically challenged BMDM. 
A
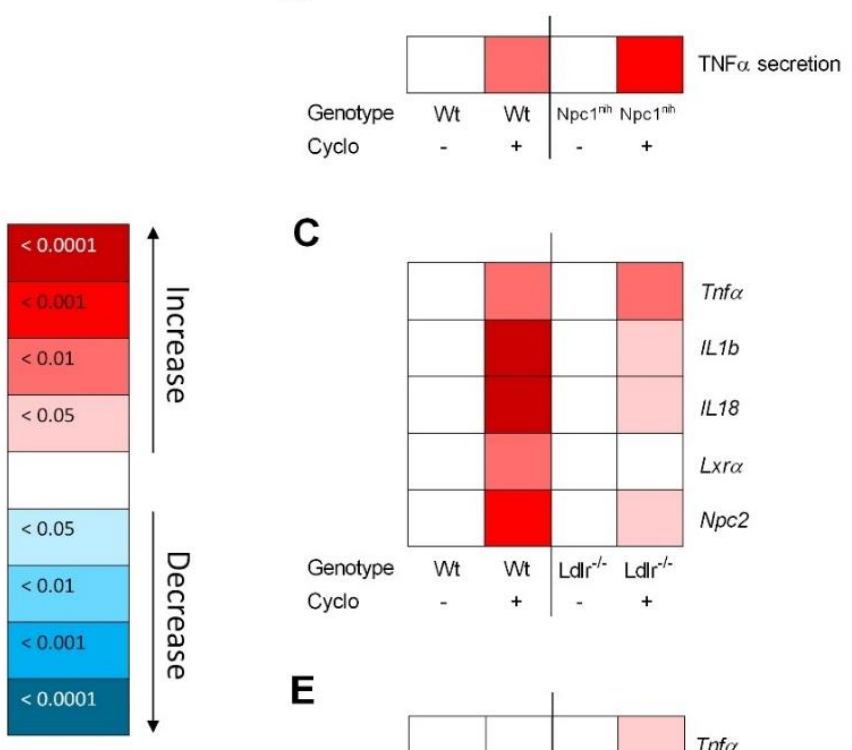

C

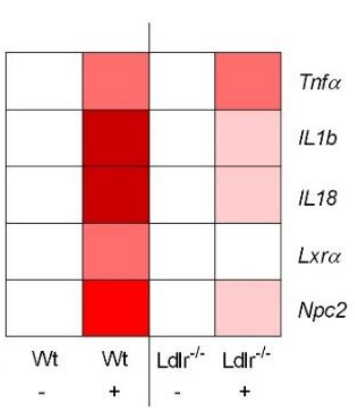

$\mathbf{E}$

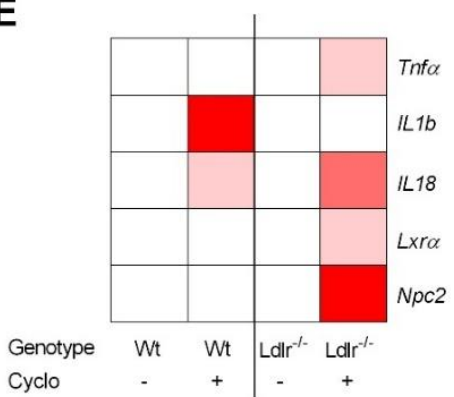

B
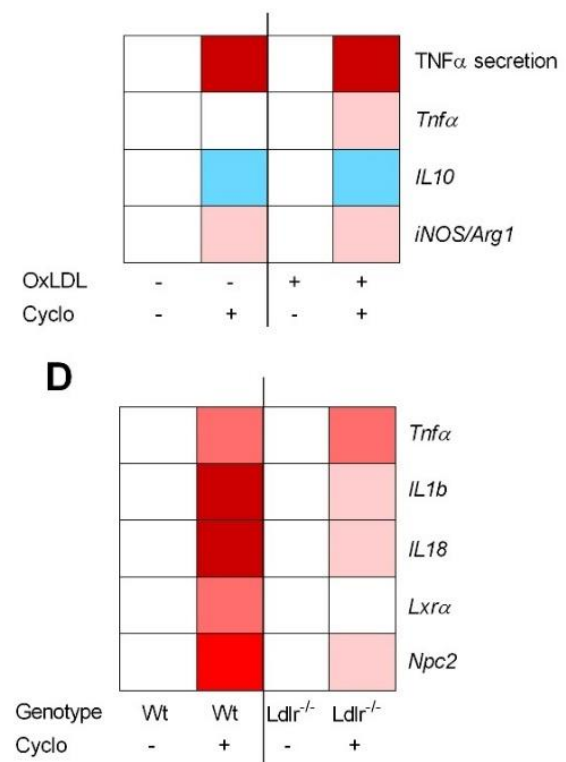

Figure 4 Metabolically challenged BMDM parameters of inflammation and cholesterol metabolism after four-hour incubation with 2-hydroxypropyl-b-cyclodextrin: (A) TNFa protein levels of 2hydroxypropyl-b-cyclodextrin $\left(1.95 \mathrm{mM}\right.$; 4hr)-treated Wt or Npc1 ${ }^{\text {nih }}$ BMDM that were terminally stimulated with LPS (100ng/ml; 4hr). (B) TNFa protein levels and Tnfa, IL10 and iNOS/Arg1 ratio gene expression data of oxLDL $(25 \mu \mathrm{g} / \mathrm{ml} ; 24 \mathrm{hr})$-exposed $N p c 1^{\text {nih }}$ BMDM treated with or without CD (4hr) that were terminally stimulated with LPS (100ng/ml; 4hr). Gene expression data were set relative to control exposed $N p c 1^{\text {nih }}$ BMDM treated with saline and stimulated with LPS. (C-E) Gene expression analysis of Tnfa, IL-1b, IL-18, Lxra and Npc2 of oxLDL $(25 \mu \mathrm{g} / \mathrm{ml} ; 24 \mathrm{hr})$-exposed $L d / r^{\circ}$ and Wt BMDM treated with or without $\mathrm{CD}(1.95 \mathrm{mM} ; 4 \mathrm{hr})$ and terminally stimulated with control (C), Pam3Cys (4hr) (D) or LPS (4hr) (E). Gene expression data were set relative to oxLDLexposed Wt BMDM treated with saline. Colored (red or blue) boxes are compared to the box directly at their left via two-way ANOVA followed by Tukey post-hoc analysis, indicating the effect of $C D$. Data are the result of 2 or 3 independent experiments.

\subsection{Incubation with 2-hydroxypropyl- $\beta$-cyclodextrin exerts pro-inflammatory effects in macrophage-derived cell lines}

To validate the inflammatory effect of $C D$ in non-primary cells, we investigated the impact of $C D$ treatment on the macrophage- derived mouse RAW 264.7 and human THP-1 cell lines. Firstly, RAW 264.7 cells were incubated with CD for 4 or $8 \mathrm{hr}$, followed by $4 \mathrm{hr}$ LPS stimulation. While $4 \mathrm{hr}$ incubation did not show any inflammatory effect, $C D$ treatment for $8 \mathrm{hr}$ increased TNFa protein secretion (Figures 5A, B; Supplementary Figures S7A, B). Furthermore, THP-1-derived macrophages were challenged with sphingomyelinase-aggregated LDL (smLDL), native LDL or control for $24 \mathrm{hr}$ and incubated with CD for $4 \mathrm{hr}$. Secreted protein levels of TNFa increased in all three experimental groups 
after $C D$ incubation (Figures $6 A-C$ ). Together, these results indicate that $C D$ also shows proinflammatory properties in macrophage-derived cell lines.

A

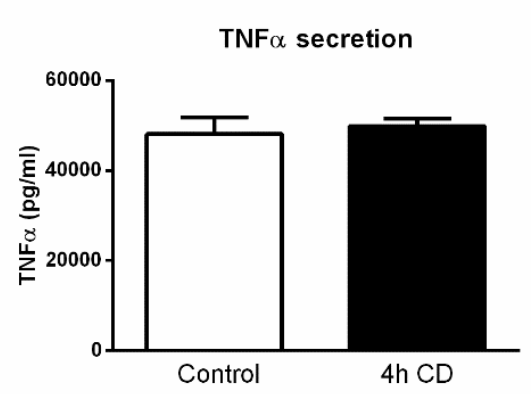

B

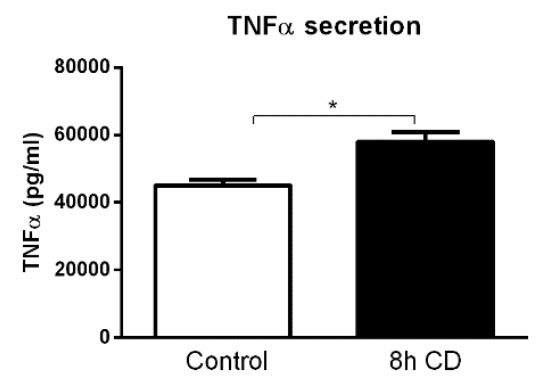

Figure 5 RAW cell line-related parameters of inflammation after prolonged exposure to 2hydroxypropyl-b-cyclodextrin: (A, B) TNFa protein levels of 2- hydroxypropyl-b-cyclodextrin (1.95 mM; 4 or $8 \mathrm{hr}$ )-treated RAW cells that were terminally stimulated with LPS (100ng/ml; $4 \mathrm{hr}$ ). Data represent $\mathrm{n}=3$ for each experimental group. ${ }^{*}$ indicates $\mathrm{p}<0.05$ by means of unpaired $\mathrm{t}$ test.

A

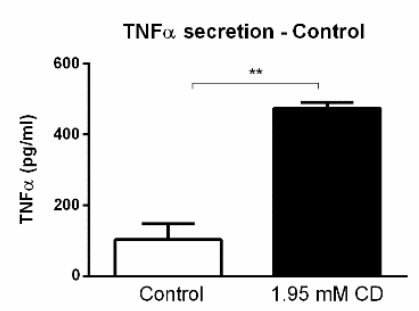

B

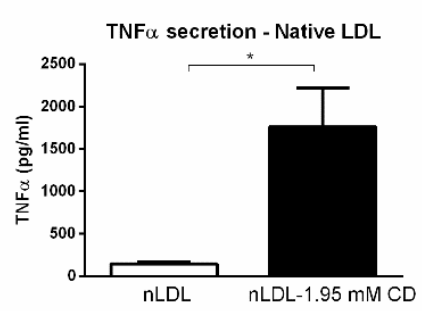

C

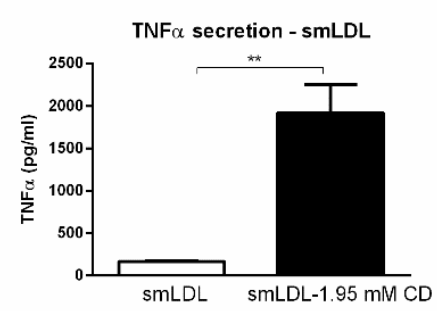

Figure 6 THP1 cell line-related parameters of inflammation after prolonged exposure to 2hydroxypropyl-b-cyclodextrin: (A-C) TNFa protein levels of control, nLDL or smLDL (24hr)exposed THP1 cells treated with or without 2-hydroxypropyl-b-cyclodextrin (1.95 mM; 4hr). *, ** indicates $p<0.05$ and 0.01 by means unpaired t-test. Data represent $n=3$ for each experimental group.

\subsection{The pro-inflammatory effect of 2-hydroxypropyl- $\beta$-cyclodextrin on bone marrow- derived macrophages is time-dependent and concentration-dependent}

As increased depletion of cholesterol from the plasma membrane was shown to result in proinflammatory responses [31], we first investigated whether incubation time influenced the proinflammatory effect of $C D$. For this purpose, wildtype BMDMs were incubated with $C D$ for 5,10 and $30 \mathrm{~min}$ as well as for 1 and $4 \mathrm{hr}$. Protein secretion of TNFa showed a marked time- dependent response after CD treatment (Figure 7A). This time-dependent, pro-inflammatory effect of CD was confirmed at gene expression levels for the inflammatory markers Tnfa, $L L-1 b$ and $C c / 2$, only showing increased expression after 1 and $4 \mathrm{hr}$ incubation (Figures 7B-D). Lxra showed a similar increase after 4 hours of CD incubation, Npc2 only showed a trend (Figures 7E, F). Expression levels of IL-18, Arg1, $A b c a 1, A b c g 1$ and Npc1 remained similar (Supplementary Figure S8). 
A

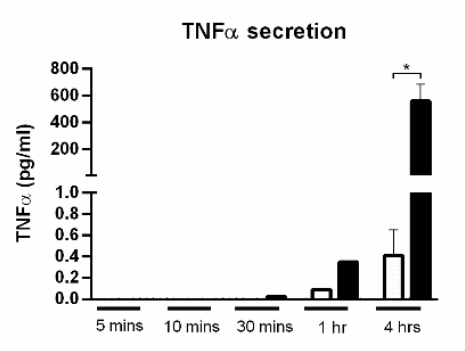

$\square \mathrm{NaCl}$
$1.95 \mathrm{mM} \mathrm{CD}$

B

C

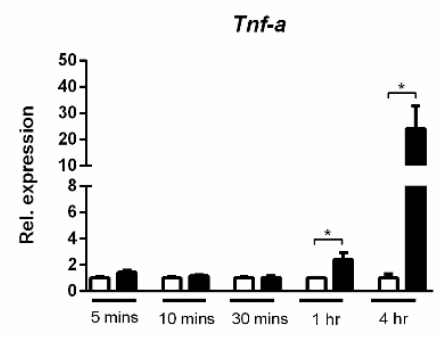

E

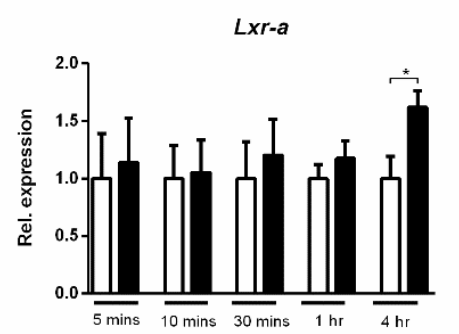

$\mu-1 b$

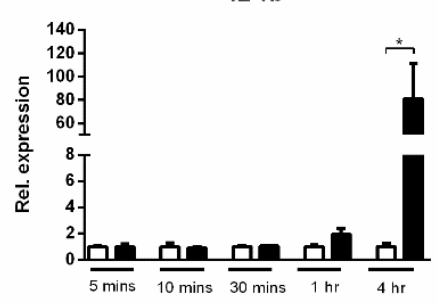

$\mathbf{F}$

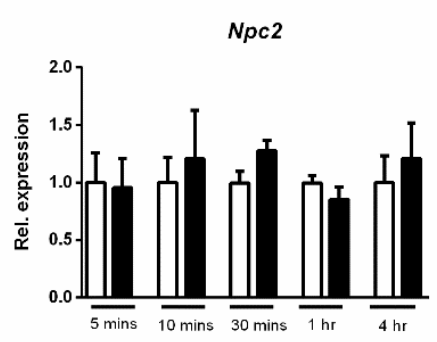

D

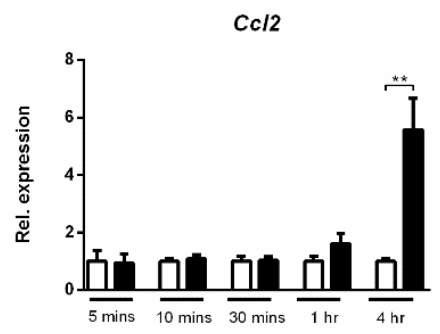

Figure 7 Time dependency of the inflammatory effect of 2-hydroxypropyl-b-cyclodextrin: (A) TNFa protein levels of Wt BMDM treated with 2-hydroxypropyl-b- cyclodextrin for different time durations. (B-D) Gene expression analysis of Tnfa, IL-1b, CCI2. (E, F) Gene expression analysis of Lxra and Npc2. Gene expression data were set relative to Wt BMDM treated with saline. Data are the result of 2 independent experiments. ${ }^{*}, *$ indicates $p<0.05$ and 0.01 by means of unpaired t-test.

To further confirm our hypothesis, we also performed a dose- response curve with CD (4hr incubation) in wildtype BMDMs. As indicated in Figure 8, CD incubation showed a clear concentration-dependent effect on TNFa secretion, adding fuel to our argument that the proinflammatory effects of $\mathrm{CD}$ are related to cholesterol depletion from the plasma membrane. 
A

TNF $\alpha$ secretion

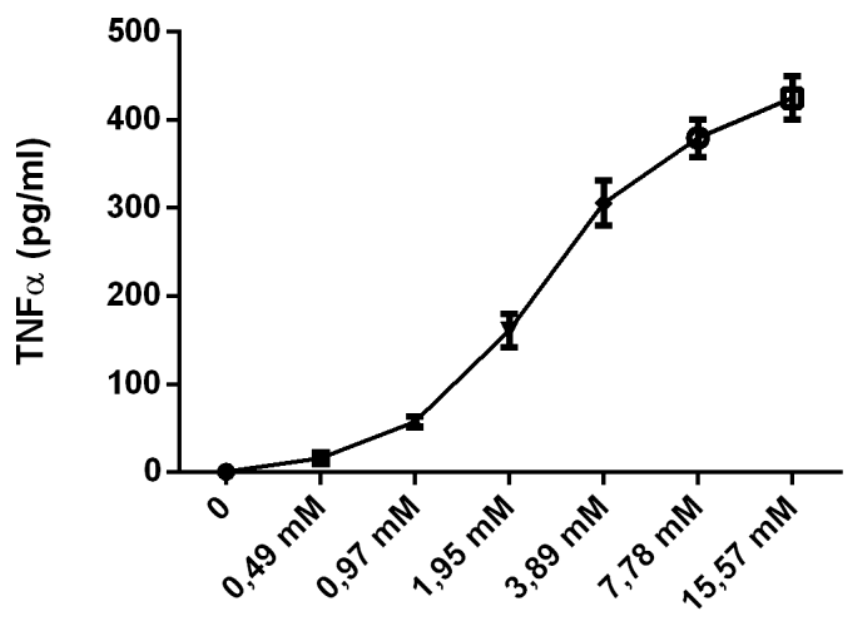

Figure 8 Concentration dependency of the inflammatory effect of 2- hydroxypropyl-b-cyclodextrin: Dose response analysis of TNFa protein levels of Wt BMDM treated with 2-hydroxypropyl-bcyclodextrin for $4 \mathrm{hrs}$.

\section{Discussion}

New perspectives to reduce cholesterol levels aim at improving cellular instead of plasma cholesterol levels, raising the argument to use $C D$ as a pharmacological compound to improve cholesterol homeostasis. Here, we confirm the cholesterol- depleting effects of CD in a metabolic inflammatory context, but concomitantly show a detrimental time- and concentration- dependent inflammatory effect of $C D$ treatment. Therefore, while $C D$ is able to decrease cellular cholesterol levels, our findings demonstrate that its use in the clinic should be closely monitored especially in patients with a metabolic inflammatory background.

Our observation that $\mathrm{CD}$ treatment promotes cellular cholesterol mobilization but induces a timeand concentration-dependent inflammatory effect implies the importance of the subcellular distribution of cholesterol and the subsequent impact of cholesterol depletion from these specific locations. Unesterified cholesterol serves a key structural function in the plasma membrane as it is critical for the formation of liquid-ordered rafts, which determine membrane fluidity. The membrane fluidity is therefore coupled to the free cholesterol/phospholipid ratio of the plasma membrane as this ratio determines the formation of the aforementioned rafts [32, 33]. Under hyperlipidemic conditions, the plasma membrane serves as the first pool to deposit free cholesterol from external sources [34]. However, accumulation of free cholesterol in the plasma membrane above an optimal free cholesterol/phospholipid ratio negatively impacts plasma membrane fluidity [33]. Indeed, YvanCharvet et al. showed that increased accumulation of free cholesterol in the plasma membrane (induced by $A b c a 1$ or $A b c g 1$ knockout) directly results in pro-inflammatory responses, supporting our current notion of the limited physiological capacity of the plasma membrane to harbor free 
cholesterol [31]. Therefore, exceeding the plasma membrane's capacity to harbor cholesterol directly results in intracellular cholesterol accumulation (mainly in lysosomes), which is considered a severe pathological phenomenon for mediating inflammatory responses [4]. Evidently, under conditions that generate severe accumulation of intracellular cholesterol (exemplified by the NPC1 mutation and LDLR knockout), CD-mediated depletion of cholesterol is highly desirable. In line with this concept, the increased lysosomal size in metabolically challenged macrophages was reverted after incubation with $C D$ treatment. This advantageous cholesterol-depleting effect of $C D$ has been confirmed in multiple previous reports $[15,18,31,35]$. In contrast, in the current study, we consistently show a harmful, pro-inflammatory (and even mild pro-fibrotic) effect of CD under cholesterol-induced inflammatory conditions in vivo and in vitro. This pro-inflammatory effect can be rationalized by the prolonged cellular exposure to $C D$, which influences plasma membrane cholesterol combined with intracellular cholesterol levels. Indeed, given the key role of cholesterol in the formation of liquid-ordered rafts, excessive depletion of plasma membrane cholesterol (as is induced with CD treatment) is highly undesirable as it disrupts these rafts, affecting membrane fluidity. This rationale is further supported by findings showing that cholesterol extraction of the plasma membrane is a primary location where CD exerts its function $[36,37]$. Therefore, excessive depletion of cholesterol from the plasma membrane by $\mathrm{CD}$ might be an explanation for the observed pro- inflammatory findings in our study as well as the time-and concentration-dependent character of our findings. The time- dependent findings are also in line with experiments reported by Pilely et al. and Ding et al., that show that while incubation of different types of cyclodextrin for a short period of time (10 and $30 \mathrm{~min}$ ) is anti-inflammatory [35], incubation for 24 and $48 \mathrm{hr}$ lead to ototoxicity [19]. Together, these observations indicate that while CD has potential in advantageously depleting cellular cholesterol, it is essential to monitor the quantity of cellular cholesterol and adjust the therapeutic dose/time accordingly to prevent undesired pro-inflammatory side-effects.

Notably, while neutrophils generally constitute the major immune cell population under inflammatory conditions, in the current study we only focused on the effect of CD on macrophages. This choice is based on previous findings by our group that demonstrate macrophages to be the most important immune cell in the in vivo model here described (as evidenced by changes in hematopoiesis [38] and organ- specific inflammation [22]). Nevertheless, as neutrophil aberrations have also been described in NPC1 disease [39], future research should further explore the involvement of neutrophils in CD-induced inflammatory responses in a metabolic inflammatory context.

These dichotomic characteristics of $C D$ raise the question whether structural manipulation of cyclodextrins can reduce the harmful pro-inflammatory effects, while maintaining the advantageous cholesterol-depleting effects of CD. Cyclodextrins are composed of cyclic oligosaccharides of 6, 7 or 8 glucose units (referred to as a-, b- and g-cyclodextrins respectively), providing cyclodextrin a polar and hydrophilic surface combined with a non-polar cavity [40]. This dual-property structure of CD grants itself for modification via polymerization, creating so-called polycyclodextrins. Indeed, various modifications have been performed on cyclodextrin, creating more efficient and effective cyclodextrins for a plethora of purposes [40]. In line, Kulkarni et al. adapted the structure CD (the type we employed in our study) and showed that their linear degradable, high molecular weight polymer variation improved the pharmacokinetic profile and bioavailability in NPC mice [41]. Moreover, using polyrotaxanes enabled specific release of $C D$ inside lysosomes, thereby minimizing the effect on plasma membrane cholesterol [42]. Based on these reports, it is anticipated that 
designing polycyclodextrins is a promising approach that can have a considerable clinical impact due to its ability to reduce the pro-inflammatory properties of $C D$ described in our study.

In conclusion, though we confirm its cholesterol-depleting effects, we here demonstrate a time- and concentration- dependent harmful pro-inflammatory effect of $C D$ under metabolic inflammatory conditions. As such, we suggest that clinical use of $C D$, in particular in a metabolic inflammatory context, should be closely monitored to prevent undesired side effects. 


\section{References}

1. van Meer, G., D.R. Voelker, and G.W. Feigenson, Membrane lipids: where they are and how they behave. Nat Rev Mol Cell Biol, 2008. 9(2): p. 112-24.

2. Demer, L.L., J.J. Hsu, and Y. Tintut, Steroid Hormone Vitamin D: Implications for Cardiovascular Disease. Circ Res, 2018. 122(11): p. 1576-1585.

3. Hegele, R.A., Plasma lipoproteins: genetic influences and clinical implications. Nat Rev Genet, 2009. 10(2): p. $109-21$.

4. Hendrikx, T., et al., Lysosomal cholesterol accumulation: driver on the road to inflammation during atherosclerosis and non-alcoholic steatohepatitis. Obes Rev, 2014. 15(5): p. 424-33.

5. Pericleous, M., et al., Wolman's disease and cholesteryl ester storage disorder: the phenotypic spectrum of lysosomal acid lipase deficiency. Lancet Gastroenterol Hepatol, 2017. 2(9): p. 670-679.

6. Santos-Lozano, A., et al., Niemann-Pick disease treatment: a systematic review of clinical trials. Ann Transl Med, 2015. 3(22): p. 360.

7. $\quad$ Defesche, J.C., et al., Familial hypercholesterolaemia. Nat Rev Dis Primers, 2017. 3: p. 17093.

8. O'Neill, S. and L. O'Driscoll, Metabolic syndrome: a closer look at the growing epidemic and its associated pathologies. Obes Rev, 2015. 16(1): p. 1-12.

9. Gehrke, N. and J.M. Schattenberg, Metabolic Inflammation-A Role for Hepatic Inflammatory Pathways as Drivers of Comorbidities in Nonalcoholic Fatty Liver Disease? Gastroenterology, 2020. 158(7): p. 1929-1947 e6.

10. Libby, P., et al., Atherosclerosis. Nat Rev Dis Primers, 2019. 5(1): p. 56.

11. Hadjiphilippou, S. and K.K. Ray, Cholesterol-Lowering Agents. Circ Res, 2019. 124(3): p. 354-363.

12. Staels, B., et al., Mechanism of action of fibrates on lipid and lipoprotein metabolism. Circulation, 1998. 98(19): p. 2088-93.

13. Schwartz, G.G., et al., Alirocumab and Cardiovascular Outcomes after Acute Coronary Syndrome. N Engl J Med, 2018. 379(22): p. 2097-2107.

14. Atger, V.M., et al., Cyclodextrins as catalysts for the removal of cholesterol from macrophage foam cells. J Clin Invest, 1997. 99(4): p. 773-80.

15. Zimmer, S., et al., Cyclodextrin promotes atherosclerosis regression via macrophage reprogramming. Sci Transl Med, 2016. 8(333): p. 333ra50.

16. Walenbergh, S.M., et al., Weekly Treatment of 2-Hydroxypropyl-beta-cyclodextrin Improves Intracellular Cholesterol Levels in LDL Receptor Knockout Mice. Int J Mol Sci, 2015. 16(9): p. 21056-69.

17. Aguisanda, F., N. Thorne, and W. Zheng, Targeting Wolman Disease and Cholesteryl Ester Storage Disease: Disease Pathogenesis and Therapeutic Development. Curr Chem Genom Transl Med, 2017. 11: p. 1-18.

18. Ory, D.S., et al., Intrathecal 2-hydroxypropyl-beta-cyclodextrin decreases neurological disease progression in Niemann-Pick disease, type C1: a non-randomised, open-label, phase 1-2 trial. Lancet, 2017. 390(10104): p. 17581768.

19. Ding, D., et al., Hydroxypropyl-beta-cyclodextrin causes massive damage to the developing auditory and vestibular system. Hear Res, 2020. 396: p. 108073.

20. Crumling, M.A., K.A. King, and R.K. Duncan, Cyclodextrins and latrogenic Hearing Loss: New Drugs with Significant Risk. Front Cell Neurosci, 2017. 11: p. 355.

21. Garcia-Mediavilla, V., et al., Effects of dietary beta-cyclodextrin in hypercholesterolaemic rats. Pharmacol Toxicol, 2003. 92(2): p. 94-9.

22. Houben, T., et al., Blood-derived macrophages prone to accumulate lysosomal lipids trigger oxLDL-dependent murine hepatic inflammation. Sci Rep, 2017. 7(1): p. 12550.

23. Houben, T., et al., Pneumococcal Immunization Reduces Neurological and Hepatic Symptoms in a Mouse Model for Niemann-Pick Type C1 Disease. Front Immunol, 2018. 9: p. 3089.

24. Bieghs, V., et al., LDL receptor knock-out mice are a physiological model particularly vulnerable to study the onset of inflammation in non-alcoholic fatty liver disease. PLoS One, 2012. 7(1): p. e30668.

25. Bieghs V, Hendrikx T, Van Gorp PJ, Verheyen F, Guichot YD, Walenbergh SM, et al. The Cholesterol Derivative 27Hydroxycholesterol Reduces Steatohepatitis in Mice. Gastroenterology (2013) 144:167-8. e161. doi: 10.1053/j.gastro.2012.09.062.

26. Bieghs, V., et al., Role of scavenger receptor $A$ and CD36 in diet-induced nonalcoholic steatohepatitis in hyperlipidemic mice. Gastroenterology, 2010. 138(7): p. 2477-86, 2486 e1-3.

27. Bieghs, V., et al., Specific immunization strategies against oxidized low-density lipoprotein: a novel way to reduce nonalcoholic steatohepatitis in mice. Hepatology, 2012. 56(3): p. 894-903.

28. Sosic-Jurjevic, B., et al., The isoflavones genistein and daidzein increase hepatic concentration of thyroid hormones and affect cholesterol metabolism in middle-aged male rats. J Steroid Biochem Mol Biol, 2019. 190: p. 1-10.

29. Houben, T., et al., Sex-opposed inflammatory effects of 27-hydroxycholesterol are mediated via differences in estrogen signaling. J Pathol, 2020. 251(4): p. 429-439.

30. Fabrice Cordelires, J.J. 3D Object Counter. 2007/06/25; Available from: http://rsbweb.nih.gov/ij/plugins/track/objects.html. 
31. Yvan-Charvet, L., et al., Increased inflammatory gene expression in ABC transporter-deficient macrophages: free cholesterol accumulation, increased signaling via toll-like receptors, and neutrophil infiltration of atherosclerotic lesions. Circulation, 2008. 118(18): p. 1837-47.

32. Simons, K. and E. Ikonen, How cells handle cholesterol. Science, 2000. 290(5497): p. 1721-6.

33. Tabas, I., Consequences of cellular cholesterol accumulation: basic concepts and physiological implications. J Clin Invest, 2002. 110(7): p. 905-11.

34. Ikonen, E., Cellular cholesterol trafficking and compartmentalization. Nat Rev Mol Cell Biol, 2008. 9(2): p. 125-38.

35. Pilely, K., et al., Alpha-cyclodextrin inhibits cholesterol crystal-induced complement-mediated inflammation: $A$ potential new compound for treatment of atherosclerosis. Atherosclerosis, 2019. 283: p. 35-42.

36. Christian, A.E., et al., Use of cyclodextrins for manipulating cellular cholesterol content. J Lipid Res, 1997. 38(11): p. 2264-72.

37. Lopez, C.A., A.H. de Vries, and S.J. Marrink, Molecular mechanism of cyclodextrin mediated cholesterol extraction. PLoS Comput Biol, 2011. 7(3): p. e1002020.

38. Magro Dos Reis I, Houben T, Gijbels MJJ, Lutjohann D, Plat J, Shiri-Sverdlov R. Anti-Inflammatory Effects of Dietary Plant Stanol Supplementation Are Largely Dependent on the Intake of Cholesterol in a Mouse Model of Metabolic Inflammation. Biomedicines (2021) 9(5):518. doi: 10.3390/biomedicines9050518.

39. Parra J, Klein AD, Castro J, Morales MG, Mosqueira M, Valencia I, et al. Npc1 Deficiency in the C57BL/6J Genetic Background Enhances Niemann-Pick Disease Type C Spleen Pathology. Biochem Biophys Res Commun (2011) 413:400-6. doi: 10.1016/j.bbrc.2011.08.096.

40. Seidi, F., Y. Jin, and H. Xiao, Polycyclodextrins: Synthesis, functionalization, and applications. Carbohydr Polym, 2020. 242: p. 116277.

41. Kulkarni, A., et al., Linear Cyclodextrin Polymer Prodrugs as Novel Therapeutics for Niemann-Pick Type C1 Disorder. Sci Rep, 2018. 8(1): p. 9547.

42. Tamura, A. and N. Yui, Polyrotaxane-based systemic delivery of beta-cyclodextrins for potentiating therapeutic efficacy in a mouse model of Niemann-Pick type C disease. J Control Release, 2018. 269: p. 148-158. 


\section{Supplemental material}

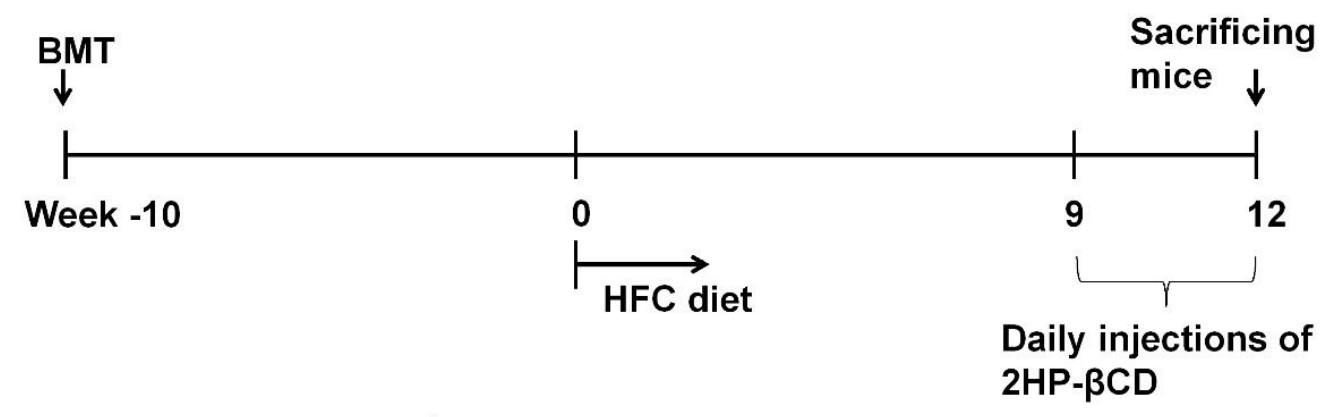

Recipients: Female $L d I r^{\prime-}$ mice

Donors: Npc1 ${ }^{\text {nih }}$-tp mice

Figure S1 Overview of experimental set-up of in vivo experiment

A

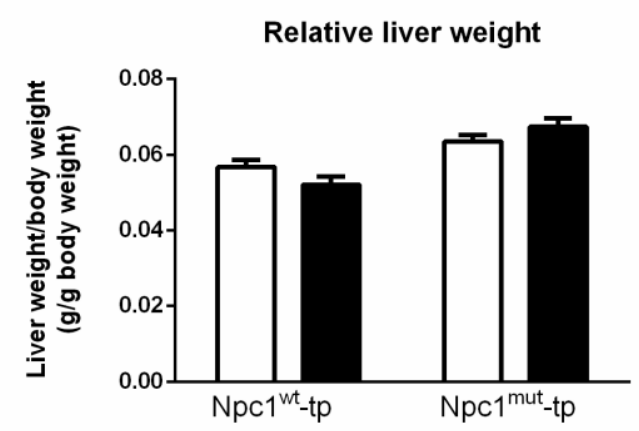

C

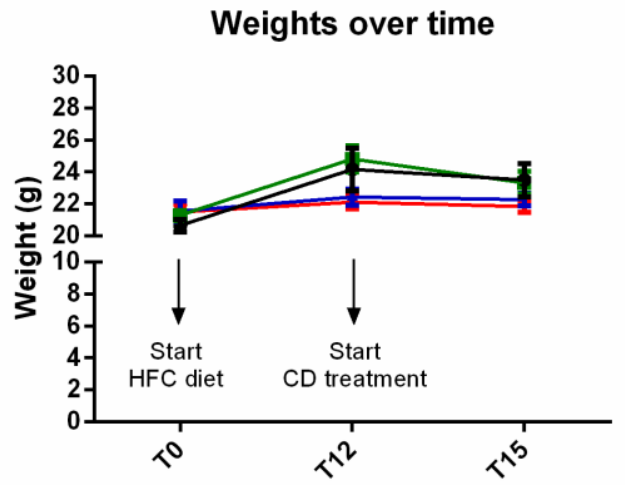

B

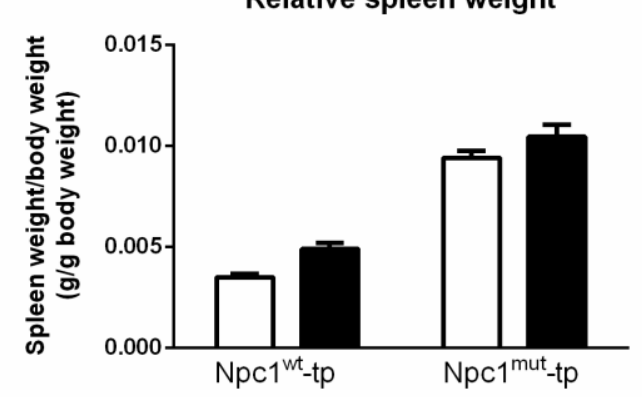

$\rightarrow N p c 1^{\text {nih }}$-tp $C D$

$\pm N$ Npc1 ${ }^{\text {nih }}$-tp

$\rightarrow N p c 1^{\text {wt }}$-tp CD

$\rightarrow \mathrm{Npc} 1^{\mathrm{wt}}$-tp

Figure S2 Relative organ weights 


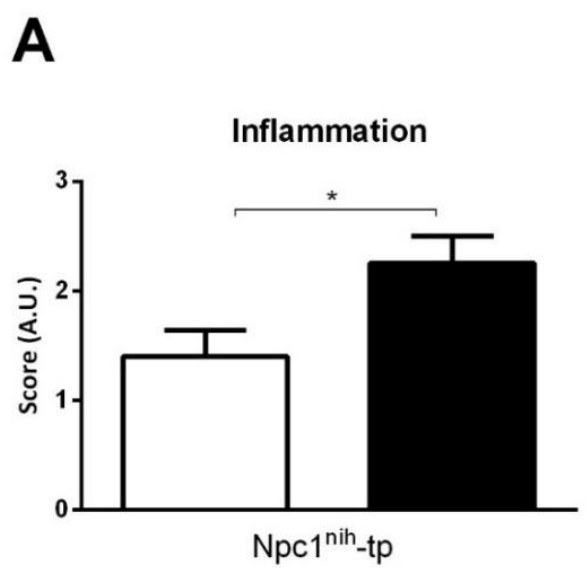

Figure S3 Quantification of hepatic HE staining of Npc1 ${ }^{\text {nih }}$-tp mice

A

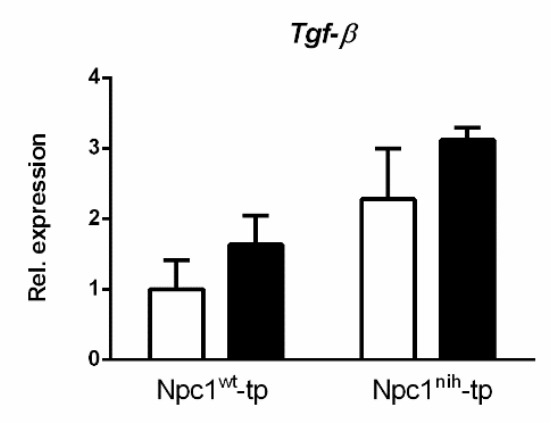

C

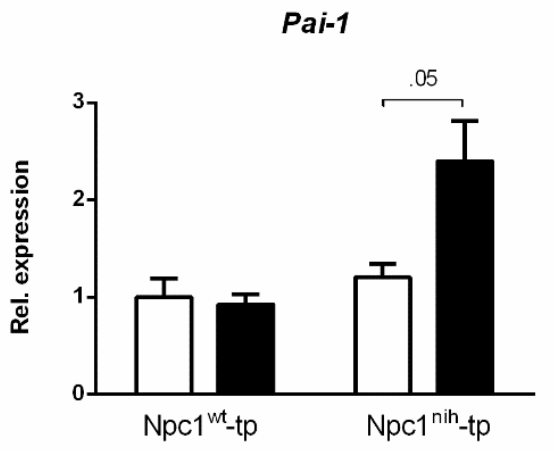

B

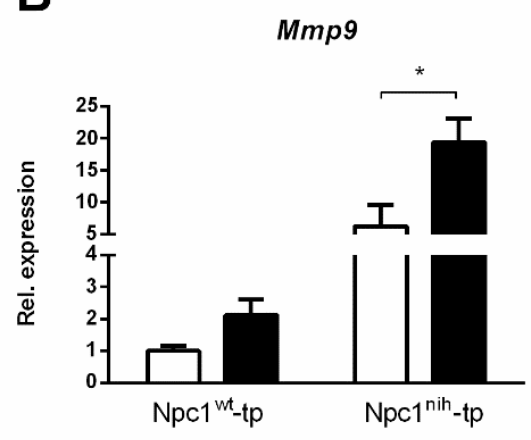

D

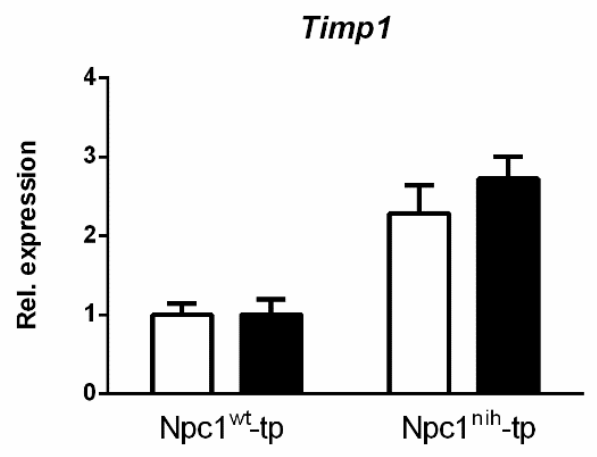

Figure S4 Hepatic gene expression of fibrotic markers 

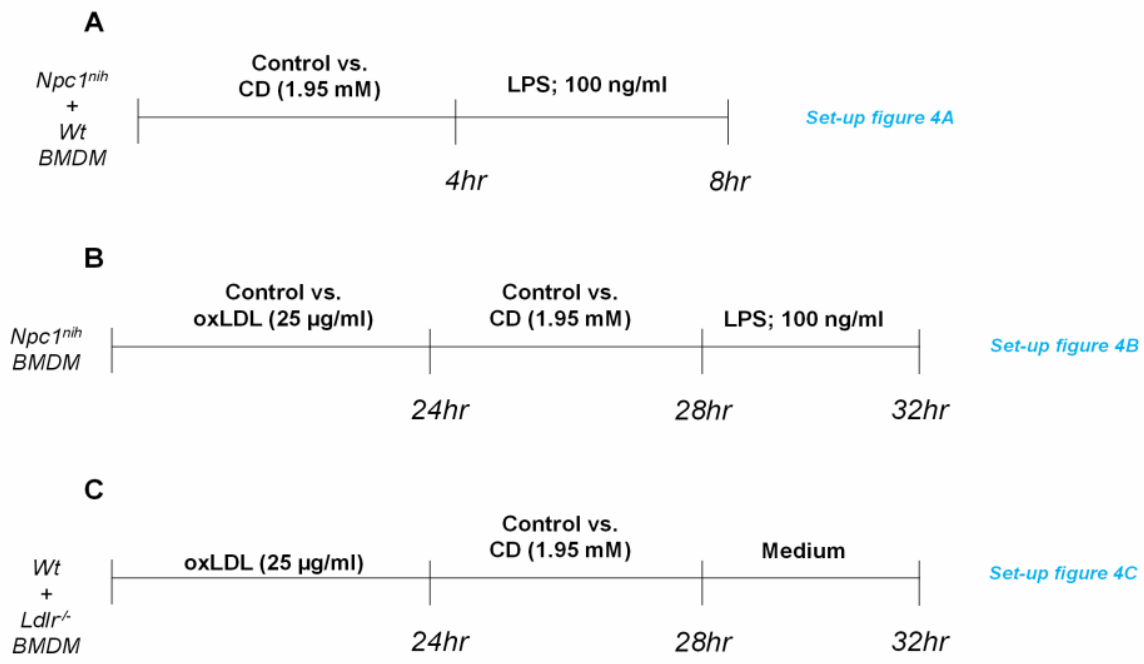

D

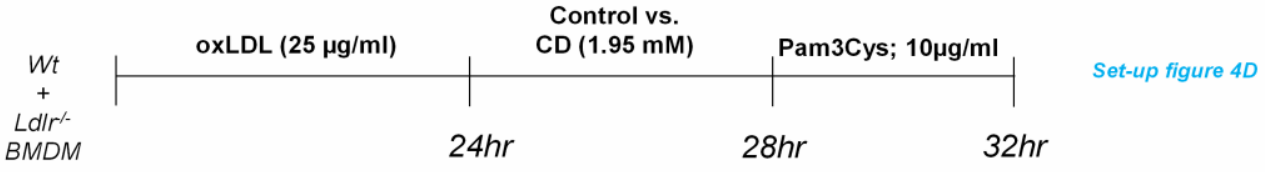

E

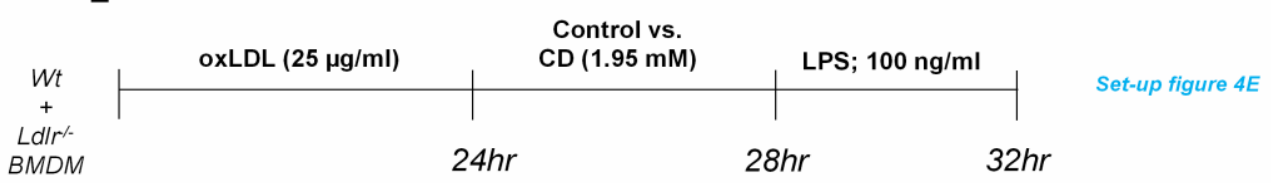

Figure S5 Experimental set-ups related to Figure 4

A

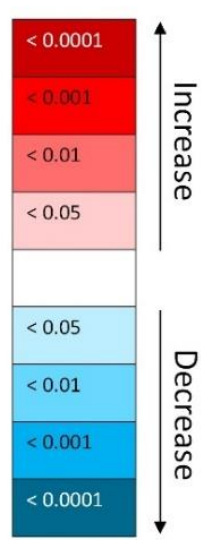

B

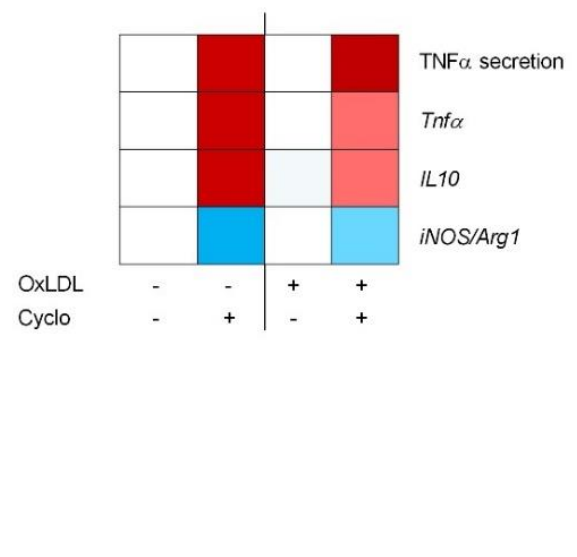

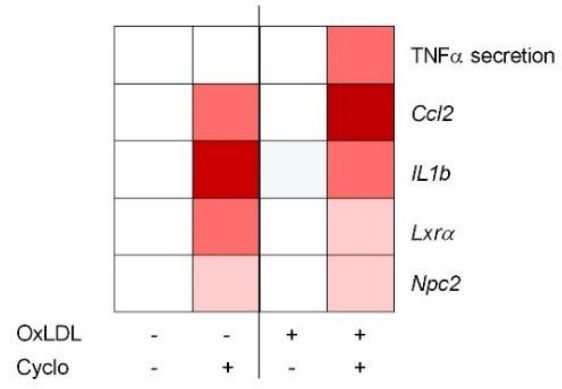

Figure S6 Heatmap of inflammatory mediators of CD-treated Wt BMDM. (A-B) TNF $\alpha$ protein levels and Tnfo, IL1b, IL-10, CCl2, Lxro and Npc2 gene expression of oxLDL (24hr)-exposed Wt BMDM treated with or without CD (4hr) that were terminally stimulated with LPS (A) or without (B) LPS for $4 \mathrm{hr}$. Gene expression data were set relative to control-exposed Wt BMDM treated with saline and stimulated with (A) or without (B) LPS. Colored (red or blue) boxes are compared to the box directly at their left via two-way ANOVA followed by Tukey post-hoc analysis, indicating the effect of CD. Data are the result of 2-4 independent experiments. 
A

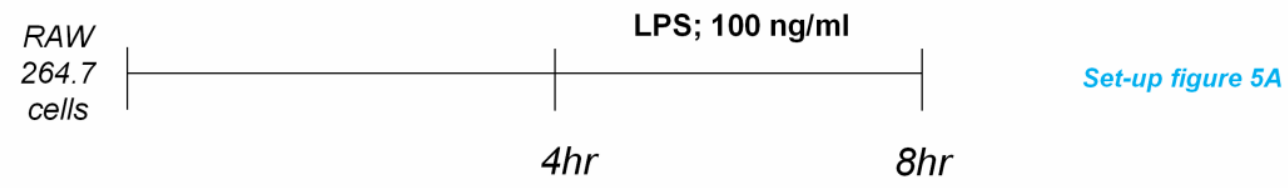

B

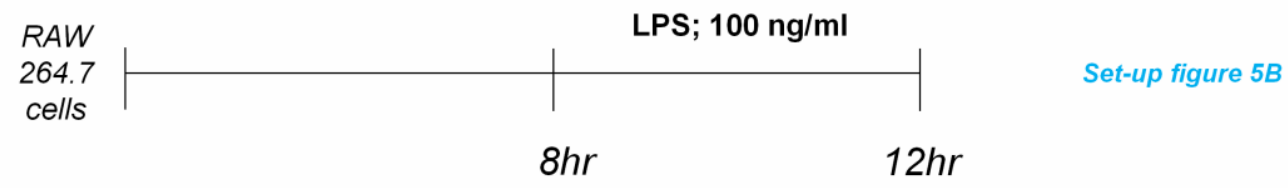

Figure S7 Experimental set-ups related to Figure 5

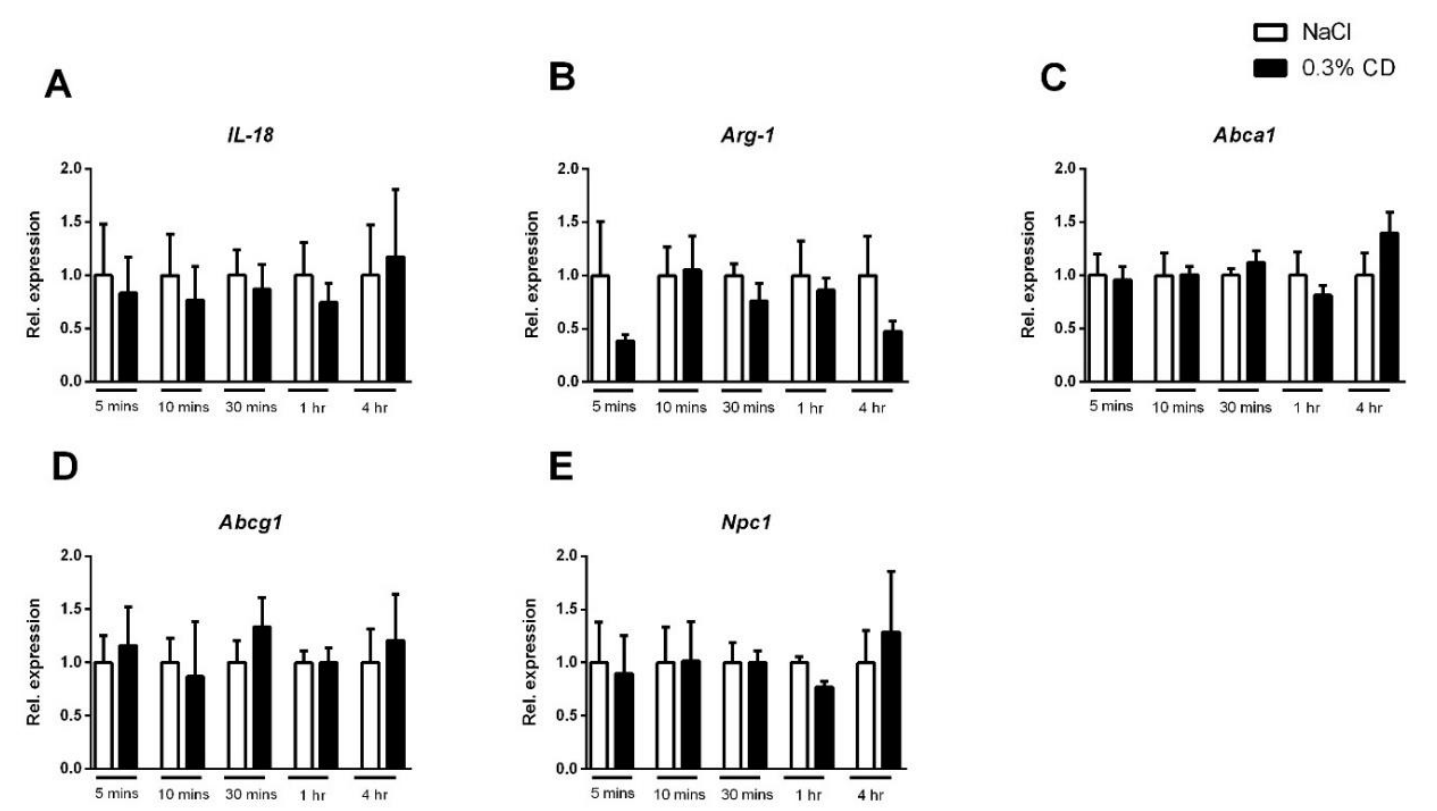

Figure S8 Gene expression analysis of BMDM exposed to different time intervals of CD 
Table S1: Primer sequences of genes used for quantitative RT-PCR.

\begin{tabular}{ccc}
\hline Gene name & Forward primer 5'-3' $^{\prime}$ & Reverse primer 5'-3' $^{\prime}$ \\
\hline Cyp7a1 & CATTACAGAGTGCTGGCCAAGA & CGCAGAGCCTCCTTGATGAT \\
\hline Cyp27a1 & CTGCACTTCCTGCTGACCAAT & AGGGCCCATGTCAGTGTGTT \\
\hline Tnfa & CATCTTCTCAAAATTCGAGTGACAA & TGGGAGTAGACAAGGTACAACCC \\
\hline Ccl2 & GCTGGAGAGCTACAAGAGGATCA & TCTCTCTTGAGCTTGGTGACAAAA \\
\hline Ccl5 & GGAGTATTTCTACACCAGCAGCAA & GCGGTTCCTTCGAGTGACA \\
\hline Saa1 & GGCTGCTGAGAAAATCAGTGATG & TCAGCAATGGTGTCCTCATGTC \\
\hline iNOS & GCAAACCCAAGGTCTACGTTCA & CCTCATTGGCCAGCTGCTT \\
\hline Arg1 & CATGGGCAACCTGTGTCCTT & CGATGTCTTTGGCAGATATGCA \\
\hline IL-10 & GCTCTTACTGACTGGCATGAG & CGCAGCTCTAGGAGCATGTG \\
\hline IL-1B & AAAGAATCTATACCTGTCCTGTGTAATGAAA & GGTATTGCTTGGGATCCACACT \\
\hline IL-18 & GACTCTTGCGTCAACTTCAAGG & CAGGCTGTCTTTTGTCAACGA \\
\hline Lxr-a & CAACAGTGTAACAGGCGCT & TGCAATGGGCCAAGGC \\
\hline Npc2 2 CACGAGCCCTGCACTTC & ACAGGGATCGGTGGGACAT \\
\hline Abca1 & CCCAGAGCAAAAAGCGACTC & GGTCATCATCACTTTGGTCCTTG \\
\hline Abcg1 & TCGGACGCTGTGCGTTTT & CCCACAAATGTCGCAACCT \\
\hline Npc1 & ATATAACGAGAGCATTCACCATGAGTA & TAACACCACGATCCCTCCAAA \\
\hline Cyclophilin (housekeeping) & TTCCTCCTTCACAGAATTATTCCA & CCGCCAGTGCCATTATGG \\
\hline Hprt (housekeeping) & TCAGTCAACGGGGGACATAAA & GGGGCTGTACTGCTTAACCAG \\
\hline
\end{tabular}

Table S2: Raw data related to confocal quantification of conditions Wt control vs. NPC1 control. Blue numbers are used for statistical analysis.

\begin{tabular}{|c|c|c|c|c|c|c|c|c|c|c|c|c|c|c|c|}
\hline \multicolumn{8}{|c|}{ WT control } & \multicolumn{8}{|c|}{ NPC1 control } \\
\hline & \multicolumn{4}{|c|}{ Absolute numbers } & \multicolumn{3}{|c|}{ Fractions (\%) } & \multicolumn{5}{|c|}{ Absolute numbers } & \multicolumn{3}{|c|}{ Fractions (\%) } \\
\hline Bmdm & $<0.1$ & $0.1-1$ & $>1$ & Sum & $<0.1$ & $0.1-1$ & $>1$ & $B m d m$ & $<0.1$ & $0.1-1$ & $>1$ & Sum & $<0.1$ & $0.1-1$ & $>1$ \\
\hline 1 & 231 & 46 & 9 & 286 & 80,77 & 16,08 & 3,15 & 1 & 157 & 28 & 40 & 225 & 69,78 & 12,44 & 17,78 \\
\hline 2 & 175 & 30 & 16 & 221 & 79,19 & 13,57 & 7,24 & 2 & 77 & 13 & 22 & 112 & 68,75 & 11,61 & 19,64 \\
\hline 3 & 137 & 49 & 18 & 204 & 67,16 & 24,02 & 8,82 & 3 & 378 & 113 & 66 & 557 & 67,86 & 20,29 & 11,85 \\
\hline 4 & 187 & 56 & 12 & 255 & 73,33 & 21,96 & 4,71 & 4 & 505 & 152 & 90 & 747 & 67,60 & 20,35 & 12,05 \\
\hline 5 & 222 & 55 & 14 & 291 & 76,29 & 18,90 & 4,81 & 5 & 237 & 61 & 46 & 344 & 68,90 & 17,73 & 13,37 \\
\hline 7 & 346 & 95 & 21 & 462 & 74,89 & 20,56 & 4,55 & & & & & & & & \\
\hline Avg & 1438 & 372 & 109 & 1919 & 74,52 & 19,37 & 6,11 & & 1491 & 390 & 297 & 2178 & 68,98 & 15,72 & 15,30 \\
\hline
\end{tabular}

Table S3: Raw data related to confocal quantification of conditions Wt control vs. Wt oxLDL. Blue numbers are used for statistical analysis.

\begin{tabular}{|c|c|c|c|c|c|c|c|c|c|c|c|c|c|c|c|}
\hline \multicolumn{8}{|c|}{ WT control } & \multicolumn{8}{|c|}{ WT oxLDL } \\
\hline & \multicolumn{4}{|c|}{ Absolute numbers } & \multicolumn{3}{|c|}{ Fractions (\%) } & \multicolumn{5}{|c|}{ Absolute numbers } & \multicolumn{3}{|c|}{ Fractions (\%) } \\
\hline$B m d m$ & $<0.1$ & $0.1-1$ & $>1$ & Sum & $<0.1$ & $0.1-1$ & $>1$ & Bmdm & $<0.1$ & $0.1-1$ & $>1$ & Sum & $<0.1$ & $0.1-1$ & $>1$ \\
\hline 1 & 231 & 46 & 9 & 286 & 80,77 & 16,08 & 3,15 & 1 & 207 & 53 & 31 & 291 & 71,13 & 18,21 & 10,65 \\
\hline 2 & 175 & 30 & 16 & 221 & 79,19 & 13,57 & 7,24 & 2 & 161 & 52 & 33 & 246 & 65,45 & 21,14 & 13,41 \\
\hline 3 & 137 & 49 & 18 & 204 & 67,16 & 24,02 & 8,82 & 3 & 209 & 42 & 61 & 312 & 66,99 & 13,46 & 19,55 \\
\hline 4 & 187 & 56 & 12 & 255 & 73,33 & 21,96 & 4,71 & 4 & 311 & 88 & 47 & 446 & 69,73 & 19,73 & 10,54 \\
\hline 5 & 222 & 55 & 14 & 291 & 76,29 & 18,90 & 4,81 & 5 & 835 & 186 & 123 & 1144 & 72,99 & 16,26 & 10,75 \\
\hline 6 & 140 & 41 & 19 & 200 & 70,00 & 20,50 & 9,50 & & & & & & & & \\
\hline 7 & 346 & 95 & 21 & 462 & 74,89 & 20,56 & 4,55 & & & & & & & & \\
\hline Avg & 1438 & 372 & 109 & 1919 & 74,52 & 19,37 & 6,11 & & 1723 & 421 & 295 & 2439 & 69,26 & 17,76 & 12,98 \\
\hline
\end{tabular}

Table S4: Raw data related to confocal quantification of conditions Wt-oxLDL-control vs. Wt-oxLDL-CD. Blue numbers are used for statistical analysis.

\begin{tabular}{|c|c|c|c|c|c|c|c|c|c|c|c|c|c|c|c|}
\hline \multicolumn{8}{|c|}{ WT oxLDL - control } & \multicolumn{8}{|c|}{ WT oxLDL -CD } \\
\hline & \multicolumn{4}{|c|}{ Absolute numbers } & \multicolumn{3}{|c|}{ Fractions (\%) } & & \multicolumn{4}{|c|}{ Absolute numbers } & \multicolumn{3}{|c|}{ Fractions (\%) } \\
\hline Bmdm & $<0.1$ & $0.1-1$ & $>1$ & Sum & $<0.1$ & $0.1-1$ & $>1$ & Bmdm & $<0.1$ & $0.1-1$ & $>1$ & Sum & $<0.1$ & $0.1-1$ & $>1$ \\
\hline 1 & 207 & 53 & 31 & 291 & 71,13 & 18,21 & 10,65 & 1 & 489 & 104 & 2 & 595 & 82,18 & 17,48 & 0,34 \\
\hline 2 & 161 & 52 & 33 & 246 & 65,45 & 21,14 & 13,41 & 2 & 301 & 43 & 2 & 346 & 86,99 & 12,43 & 0,58 \\
\hline 3 & 209 & 42 & 61 & 312 & 66,99 & 13,46 & 19,55 & 3 & 509 & 123 & 21 & 653 & 77,95 & 18,84 & 3,22 \\
\hline 4 & 311 & 88 & 47 & 446 & 69,73 & 19,73 & 10,54 & 4 & 368 & 81 & 8 & 457 & 80,53 & 17,72 & 1,75 \\
\hline \multirow[t]{2}{*}{5} & 835 & 186 & 123 & 1144 & 72,99 & 16,26 & 10,75 & 5 & 271 & 76 & 15 & 362 & 74,86 & 20,99 & 4,14 \\
\hline & & & & & & & & 6 & 383 & 107 & 51 & 541 & 70,79 & 19,78 & 9,43 \\
\hline Avg & 1723 & 421 & 295 & 2439 & 69,26 & 17,76 & 12,98 & & 2321 & 534 & 99 & 2954 & 78,88 & 17,87 & 3,24 \\
\hline
\end{tabular}


Table S5: Raw data related to confocal quantification of conditions NPC1 control vs. NPC1 CD. Blue numbers are used for statistical analysis.

\begin{tabular}{|c|c|c|c|c|c|c|c|c|c|c|c|c|c|c|c|}
\hline \multicolumn{8}{|c|}{ NPC1 - control } & \multicolumn{8}{|c|}{ NPC1 -CD } \\
\hline & \multicolumn{4}{|c|}{ Absolute numbers } & \multicolumn{3}{|c|}{ Fractions (\%) } & \multirow[b]{2}{*}{$B m d m$} & \multicolumn{4}{|c|}{ Absolute numbers } & \multicolumn{3}{|c|}{ Fractions (\%) } \\
\hline Bmdm & $<0.1$ & $0.1-1$ & $>1$ & Sum & $<0.1$ & $0.1-1$ & $>1$ & & $<0.1$ & $0.1-1$ & $>1$ & Sum & $<0.1$ & $0.1-1$ & $>1$ \\
\hline 1 & 157 & 28 & 40 & 225 & 69,78 & 12,44 & 17,78 & 1 & 157 & 51 & 7 & 215 & 73,02 & 23,72 & 3,26 \\
\hline 2 & 77 & 13 & 22 & 112 & 68,75 & 11,61 & 19,64 & 2 & 428 & 103 & 23 & 554 & 77,26 & 18,59 & 4,15 \\
\hline 3 & 378 & 113 & 66 & 557 & 67,86 & 20,29 & 11,85 & 3 & 205 & 54 & 11 & 270 & 75,93 & 20,00 & 4,07 \\
\hline 4 & 505 & 152 & 90 & 747 & 67,60 & 20,35 & 12,05 & 4 & 208 & 58 & 9 & 275 & 75,64 & 21,09 & 3,27 \\
\hline 5 & 237 & 61 & 46 & 344 & 68,90 & 17,73 & 13,37 & 5 & 248 & 66 & 34 & 348 & 71,26 & 18,97 & 9,77 \\
\hline Avg & 1491 & 390 & 297 & 2178 & 68,98 & 15,72 & 15,30 & & 1395 & 360 & 102 & 1857 & 74,92 & 19,45 & 5,63 \\
\hline
\end{tabular}

Supplementary video 1: Wildtype control

Supplementary video 2: $N p c 1^{\text {nih }}$ control

Supplementary video 3 : Wildtype oxLDL

Supplementary video 4: Wildtype oxLDL CD

Supplementary video 5: $N p c 1^{\text {nih }} \mathrm{CD}$

\section{Supplemental methods}

\section{Macro created to quantify volumes and coordinates of lysosomes}

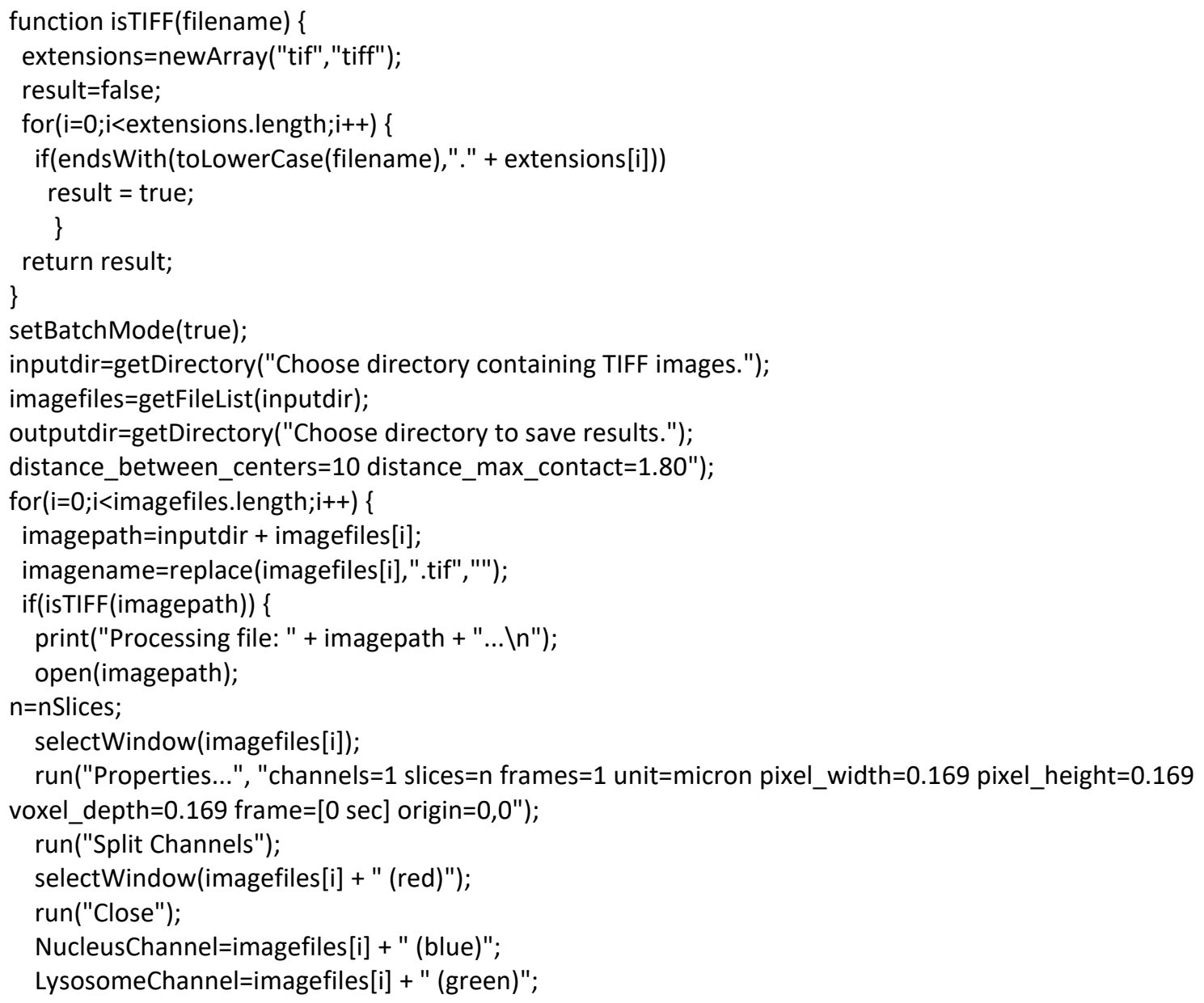




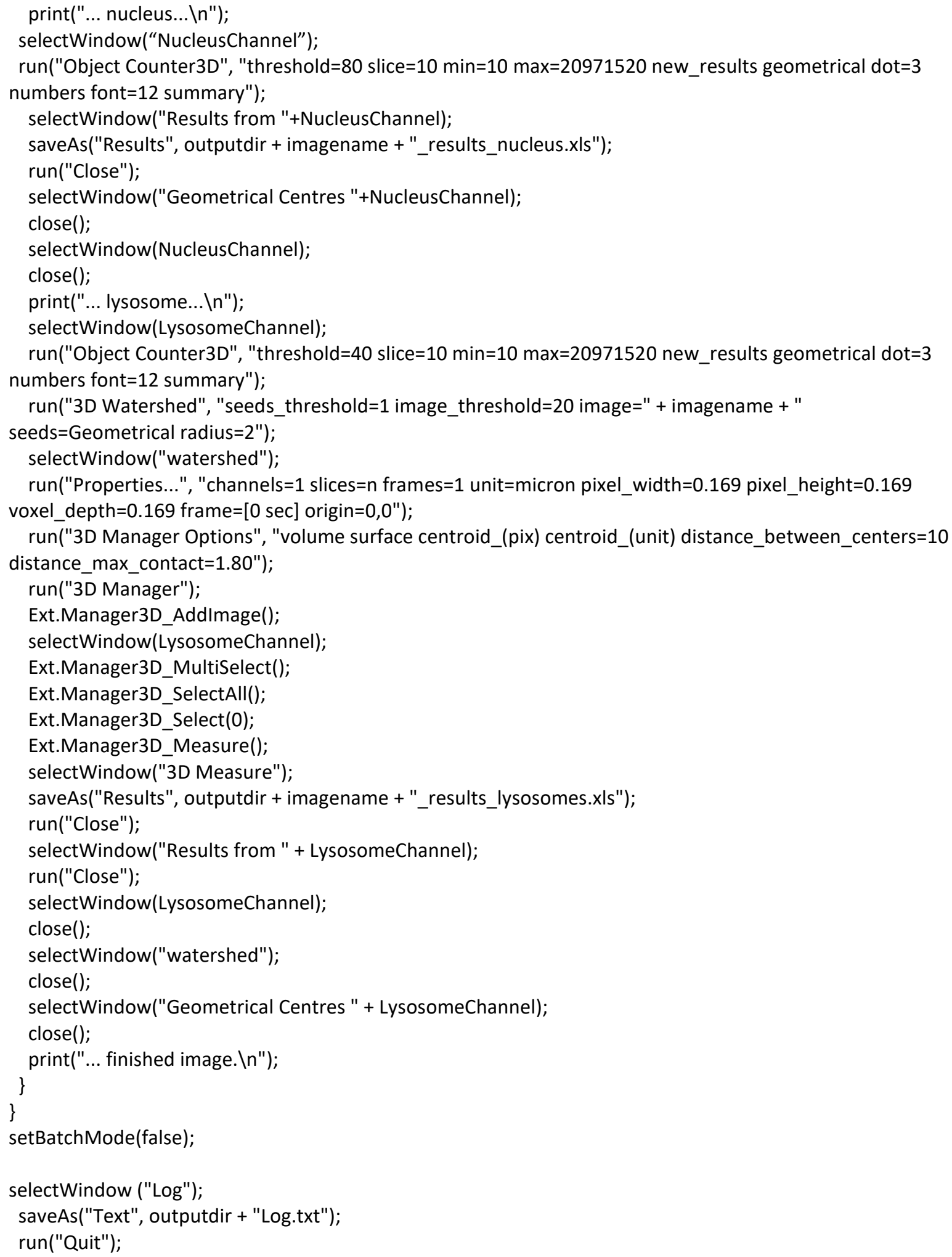




\section{Chapter 7}

General discussion 
Inflammation is considered a strong risk factor in the development of many diseases related to the metabolic syndrome such as NAFLD, diabetes, cardiovascular diseases and cancer. However, the exact cause of inflammation and the underlying mechanisms in these diseases are not yet completely understood. In the current thesis, we aimed to characterize the mechanisms linking lysosomal lipid overload and inflammation and investigated the effects of different intervention strategies to treat metabolic inflammation. One of such interventions include the potential of the lysosomal protease, cathepsin D, as a safe therapeutic target in metabolic inflammatory diseases. Here, we bring together the results of the chapters to discuss the implications of our findings on the future potential of different therapeutic approaches in modulating inflammation.

\section{Secretion of Cathepsin D is increased upon various external and internal stimuli and induces inflammation}

Metabolic and inflammatory pathways are tightly connected, meaning that metabolic burden induces lipid metabolism regulators that influence inflammatory processes [1,2]. Under normal conditions, excess cholesterol is taken up by macrophages and is processed inside the lysosome. Reesterified cholesterol is transported back to the cytoplasm where it is stored or exported out of the cell by cholesterol efflux transporters [3,4]. Cholesterol efflux is regulated by liver $X$ receptors (LXRs) that not only detect intracellular cholesterol overload but also activate genes involved in antiinflammatory response [5]. Dysregulation of metabolism results in loss of lipid control leading to cholesterol accumulation, oxidative stress and insulin resistance $[6,7]$. Relevantly, macrophage lipid accumulation and defective lysosomal function are indicated as central events in many metabolicinflammatory disorders. Unfavorable accumulation of lipids in tissues and high levels of circulating lipids, which are prone to oxidation, are commonly associated with inflammatory conditions $[8,9]$. Specifically, oxidation of Low-Density Lipoprotein (oxLDL) particles is considered as a marker of inflammation [10]. In line, we have formerly shown that intracellular accumulation of oxLDL in hepatic macrophages causes inflammation in non-alcoholic steatohepatitis (NASH) [11]. Importantly, recent data showed that plasma levels of the lysosomal protease, cathepsin D (CTSD) correlated with total cholesterol levels and severity of hepatic inflammation in patients [12]. This observation suggests a link between lipid excess, secretion of CTSD and inflammation.

In Chapter 2, we have extensively reviewed the triggers and respective mechanisms through which lysosomal cathepsins are released into cytosol and extracellular space. Progressive accumulation of modified lipids such as oxLDL in macrophages are known to cause trafficking defects that disrupt the lysosomal membrane integrity leading to release of lysosomes enzymes into the plasma $[13,14]$. The released lysosomal enzymes and undigested lipids can trigger immune responses. The discharge of CTSD has been reported to affect various pathways which can result in the initiation of inflammatory responses. Secreted pro-CTSD by its proteolytic activity on extra- and/or transmembrane proteins [15], by disturbed autophagy [16] or by initiating apoptosis [17,18], as well as by damaging extracellular matrix (ECM) [19] can lead to inflammation. For instance, macrophages are capable of responding to external signals by secretion of CTSD which is associated with inflammatory responses in IBD [20], cancer [21,22] and cardiovascular diseases [23]. However, despite the numerous associations of CTSD, in particular extracellular CTSD with inflammatory processes, it has not yet been demonstrated whether extracellular CTSD has a direct effect on inflammation. In Chapter $\mathbf{3} \boldsymbol{\&} \mathbf{4}$ this was investigated through the use of specific inhibition of extracellular CTSD which resulted in 
improved lipid metabolism and reduced inflammation in high fat-fed rodent models suggesting that pro-CTSD secretion is involved with mediating metabolic inflammatory responses.

Apart from lipid mediators, acute and chronic stress are also associated with increased inflammatory activity. In chapter 5, we have described in detail the relation between stress/gut microbiota/brain interplay in intestinal barrier dysfunction and inflammation. Intestinal macrophages of inflamed mucosa are known to induce cathepsin secretion resulting in further tissue destruction and inflammation [24]. To note, the impact of stress and gut microbiota on CTSD function was not tested in the current thesis and future studies measuring the levels and activity of CTSD upon these stimuli are needed to confirm these observations. Collectively, the findings of this thesis suggest that CTSD release from cells upon external and internal stimuli seems to be a key process in inflammatory signaling.

\section{Extracellular cathepsin $D$ has a distinct pathophysiological role in inflammation compared to its lysosomal counterpart}

In chapter 2 \& 4, we have described that the intracellular and extracellular CTSD fractions have distinct cellular and molecular functions. Our findings demonstrated that the respective fractions of CTSD exhibit a different proteome profile and thereby regulate distinct cellular pathways. CTSD is an aspartic protease which is synthesized and translocated into the endoplasmic reticulum as an inactive pre-proCTSD ( $52 \mathrm{kDa}$ ) which is converted into an enzymatically active, intermediate pro-CTSD (48 $\mathrm{kDa}$ ) that is finally processed into the mature CTSD form of $32 \mathrm{kDa}$ in the lysosomes $[15,25]$. Lysosomal CTSD is involved in the processing of antigenic peptides [26], hormones [27,28], and growth factors [29]. Failure of such functions either by mutations or partial loss of CTSD activity is known to cause abnormal accumulation of cholesterol esters [30], lysosomal malfunction [31], neurodegeneration [32], developmental regression, visual loss [33]. CTSD knock-out mice are thus short-lived [34,35]. Under physiological conditions, extracellular CTSD is mainly involved in ECM degradation [36] and hydrolysis of protein components of LDL [37]. A small fraction of pro-CTSD is secreted under normal conditions and is found in biological fluids such as blood, sweat [38] and urine [39]. However, pro-CTSD is secreted in large quantities in several types of cancer [40], NASH [12], diabetes [41] and IBD [20], implying its role in metabolic pathologies.

The observed differences in the functionalities of intracellular and extracellular CTSD fractions might stem from a variety of factors including the $\mathrm{pH}$ of the microenvironment, availability of interacting partners or differences in the post-translational modifications of extracellular pro-CTSD and active lysosomal CTSD fractions. For instance, secreted pro-CTSD from MCF-7 cells harbored different glycosylation patterns rendering it sensitive to Endo $\mathrm{H}$ (glycosidase) digestion, whereas lysosomal CTSD from healthy tissues is partially Endo H-resistant which might affect its functions [25]. Moreover, there is evidence that CTSD displays both proteolytic-dependent and independent actions. Indeed, pro-CTSD has been shown to have the potential to act as a protease by cleaving various substrates as well as a ligand (binding function) for membrane receptors [42-44]. A CTSD variant (mutation at $\mathrm{D} 231 \mathrm{~N}$ ) that lacks the catalytic function was still shown to be mitogenic in fibroblasts, indicating a pathway involving protein-protein interaction [45]. Thus, pro-CTSD might govern a greater number of functions than the lysosomal fraction due to its dual function as a ligand and a protease. Given the site-specific role for CTSD, inhibitors specifically targeting extracellular CTSD, and/or intracellular CTSD and tools that can block its protein binding functions are favored. 
The small-molecule inhibitor used in our experiments does not pass through the cell membrane and therefore is retained extracellularly. With this approach, extracellular action of CTSD is selectively inhibited, but the crucial intracellular function of CTSD was undisturbed.

In Chapter 3 \& 4, we showed reduced hepatic steatosis, insulin resistance, decreased expression of pro-inflammatory cytokines and induction of an anti-inflammatory state in rodent models upon selectively blocking the activity of extracellular CTSD. Extracellular CTSD is known to process several lipoprotein receptors and lipid transporters and thereby regulate lipid metabolism. It modifies apolipoprotein B-100 (apoB) component of the LDL molecules leading to their accumulation [46] and directly interacts with low-density lipoprotein receptor-related protein 1 (LRP1) [47] thereby modulating ATP-binding cassette transporter A1 (ABCA1) expression [48]. Xian and co-workers proposed an elegant model through which they demonstrated that macrophage LRP1 not only controls ABCA1 expression to sustain cholesterol efflux, but also incorporates cellular cholesterol homeostasis with inflammation [49]. In the setting of lipid overload, binding of oxLDL to CD-36/TLR4 complex initiates MyD88 dependent inflammation. To counteract this, LRP1 promotes ABCA1 gene expression by Shc1/PI3K/Akt/Ppar- $\gamma /$ Lxr- $\alpha$ axis to mediate cholesterol export. Besides cholesterol efflux, ABCA-1 modulates TLR-4 trafficking to membrane and induces LXRs to inhibit inflammatory pathways downstream of TLRs $[50,51]$. In our experiments, extracellular CTSD inhibition altered the expression of CD36 and SR-A, reduced pro-inflammatory cytokine expression in macrophages, upregulated PPAR signaling and downregulated the TLR-4 pathway, suggesting that extracellular CTSD disrupts the LRP1/TLR-4 axis to propagate inflammation (Chapter 4) as illustrated in Figure 1. 


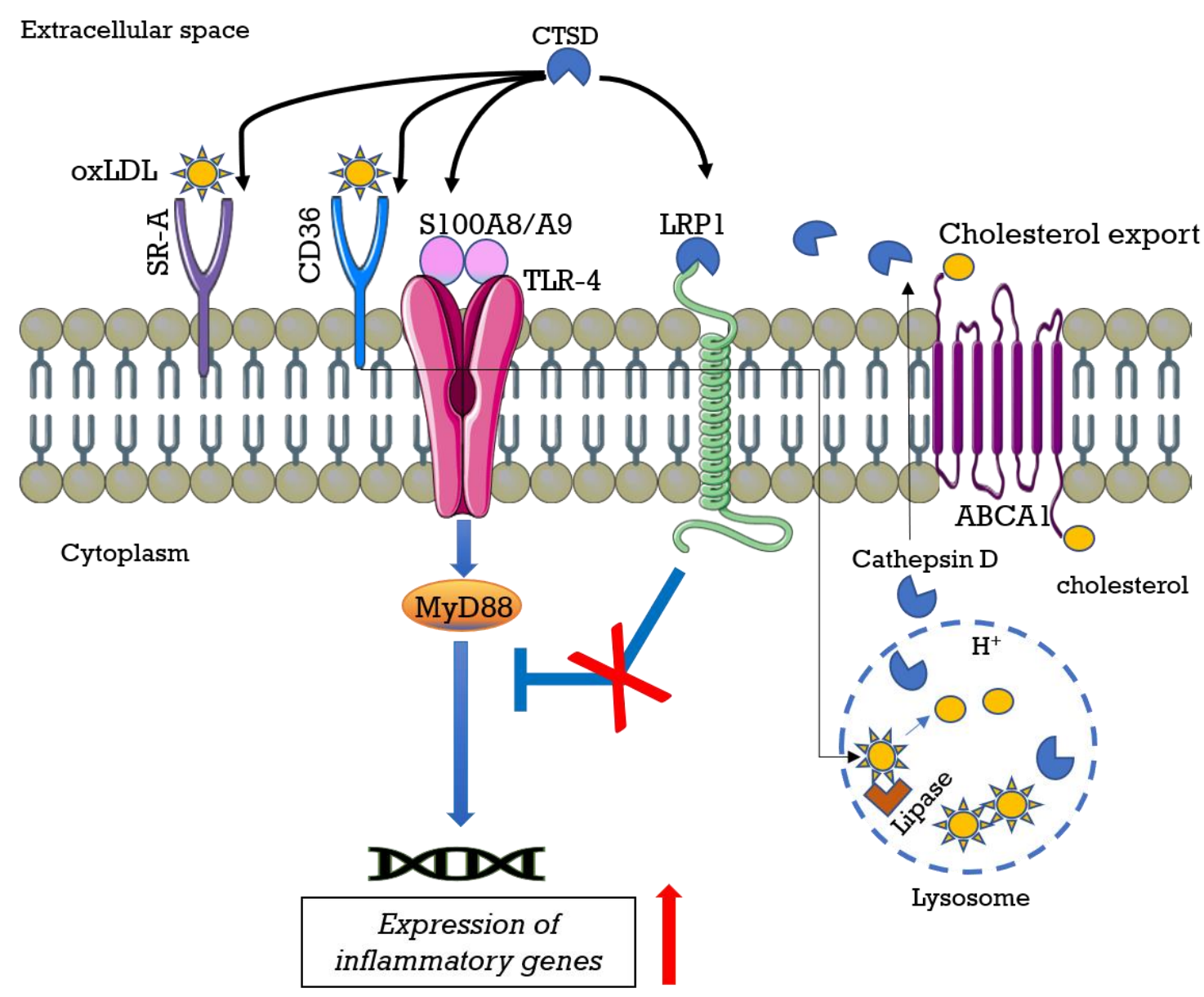

Figure 1 Proposed mechanism of extracellular CTSD action. Under normal conditions, oxLDL is endocytosed by the cells using CD36/SR-A receptors and is sent to lysosomes for subsequent degradation. During hyperlipidemic conditions, excess oxLDL bound to CD-36 can elicit TLR-4 MYD88 dependent inflammatory responses. To suppress this, LRP1 mediates crosstalk between ABCA1 and TLRs to increase cholesterol efflux and cease inflammation respectively. Accumulation of oxLDL inside lysosomes leads to lysosomal membrane permeabilization and release of CTSD. The interaction of extracellular CTSD with LRP1 blocks LRP1 signaling leading to increased inflammatory gene expression. In our experiments, inhibition of extracellular CTSD altered the expression of CD36, SR-A and TLR4 suggesting that extracellular CTSD acts as a master regulator of CD36/TLR-4/LRP1 axis in promoting inflammation. Several elements of the figure are taken from Servier Medical Art image bank.

Moreover, it is possible that increased CTSD activity is both a cause and consequence of inflammatory activity, i.e., that the relationship between CTSD and inflammation is bi-directional where one factor activates the other. In line, inflammatory cytokines are known to cause the secretion of pro-CTSD in endothelial cells [52]. An additional mechanism through which extracellular CTSD may participate in inflammation is via degradation of ECM. The inflammatory process involved in IBD, cardiovascular diseases and cancer appear to converge at the step of ECM degradation by extracellular cathepsins [53]. In IBD patients, increased production and secretion of CTSD participates in ECM degradation of the mucosa leading to damage of intestinal barrier $[20,54]$. CTSD from the tumor-conditioned media was able to degrade ECM proteins exacerbating tumorigenesis $[55,56]$. In line, our proteomics data showed the downregulation of ECM proteins such as collagen, prolargin and xylosyl transferase 2 upon extracellular CTSD inhibition, suggesting that CTSD might mediate inflammatory responses by 
degrading ECM components (Chapter 4). Although this thesis demonstrated a clear effect of extracellular CTSD on inflammation/lipid metabolism, the exact downstream effectors of CTSD are unknown. Therefore, future studies using yeast two-hybrid screening or mutagenesis approaches are needed to identify the binding partners of CTSD in the extracellular space.

\section{Alternative therapeutic targets for modulating cholesterol-induced metabolic inflammation}

The involvement of lysosomal cholesterol accumulation as one of the primary causal factors in inflammatory conditions, makes it an attractive therapeutic target. Therefore, in chapter 6, we evaluated the potential effects of 2-hydroxypropyl- $\beta$-cyclodextrin (CD), a cholesterol-mobilizing compound in models of metabolic inflammation. We found that CD intervention in both in vitro and in vivo models of metabolic inflammation led to cholesterol mobilization, but with an accompanying unexpected increase in inflammation. The observed pro-inflammatory effects of $C D$ could be the result of its cholesterol-depleting properties. CDs normalize cholesterol homeostasis by releasing the trapped unesterified cholesterol from membranes and also facilitate the cholesterol egress from lysosomal compartments by enhancing lysosomal exocytosis $[57,58]$. Depletion of plasma membrane cholesterol can lead to dispersal of lipid rafts and associated plasma membrane proteins [59] and affect the function of membrane receptor complexes [60] which are processes known to induce inflammation. This observation is also in line with our observed time-dependent pro-inflammatory effect of $C D$, suggesting that short exposure to $C D$ s is still beneficial. As discussed in the previous section, lipid raft dispersal can initiate MyD88/NF-KB activation and subsequent inflammation. Indeed, CD was shown to activate the innate immune response through these lipid-dependent biological processes [61]. Besides cholesterol depletion, lysosomal exocytosis can also lead to propagation of inflammatory responses as discussed in chapter $\mathbf{2}$. Hence, it is plausible to assume that $\mathrm{CD}$ treatment would change the mechanical properties of the membranes leading to the proinflammatory responses. Therefore, it is important to monitor the clinical application of $C D$ to prevent unwanted side effects. Altogether, our findings demonstrate a clear and well-defined connection between lysosomal cholesterol accumulation and inflammation. The summary of the main findings of this thesis are represented in Figure $\mathbf{2}$. 


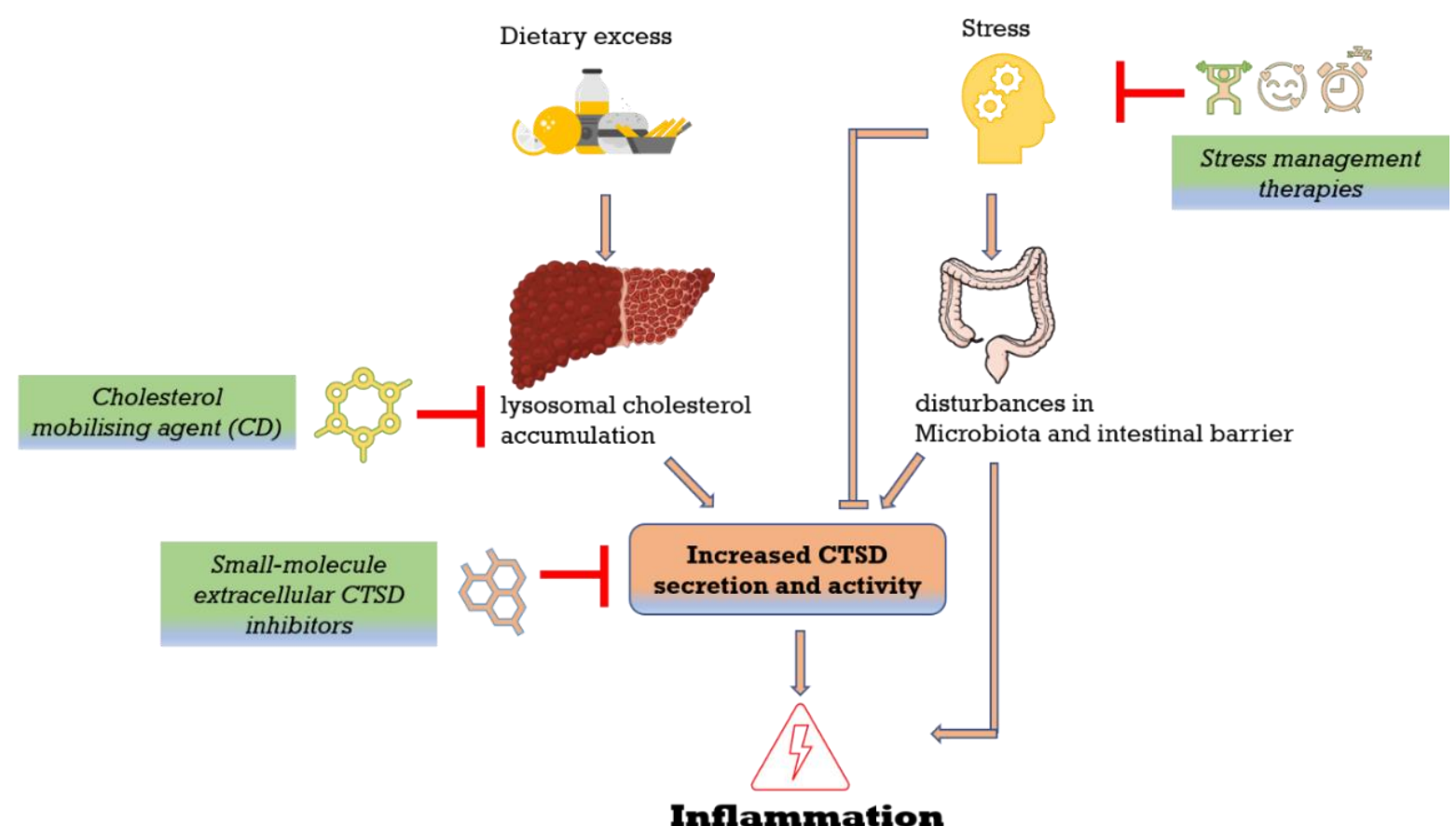

Figure 2 Summary of the main findings. Consumption of high fat diet can lead to lysosomal cholesterol accumulation and subsequent destabilization of the lysosomal membrane leading to release of CTSD into extracellular space. Inhibition of oxLDL-induced CTSD decreased hepatic steatosis and inflammation (chapter 3 \& 4). Alternatively, lysosomal cholesterol accumulation was reduced by $C D$ intervention (chapter 6). In addition to dietary factors, stress can have impact on gut microbiota composition leading to inflammatory responses in IBD by releasing lysosomal enzymes or by disturbing intestinal barrier and therefore stress management options can help alleviate IBD symptoms (chapter 5). Figure is created with permission from Servier Medical Art image bank.

\section{Clinical implications of the findings}

Dietary supplements and life-style interventions including exercise and stress management have produced mixed results in treating inflammation in patients with NAFLD [62] and IBD [63, 64]. In addition, the anti-inflammatory effects of various medications are inconclusive, possibly due to partial effects on metabolic dysregulation or due to the fact that the cause of inflammation can be multifactorial in these diseases [65]. Moreover, the complex pathophysiology and heterogenous patient populations makes the drug development particularly challenging. This further rationalizes the development of innovative pharmacologic therapeutics aimed at specific molecular targets in inflammatory disorders.

Although cathepsins have proven to be appealing targets in osteoporosis, atherosclerosis and cancer, their clinical adaption is often hindered by the 'off-target' effects and adverse responses [66]. However, progress in understanding the structure of the cathepsins' active sites with parallel advances in computational and in silico modelling allowed the development of potent selective cathepsin inhibitors with minimal or zero negative effects. In the current thesis, we demonstrated that targeted inhibition of extracellular CTSD activity restores dysregulated lipid metabolism and reduces inflammation both in vitro and in vivo (Chapter 3 \& 4). However, validation of these findings 
using human samples is needed to successfully translate these pre-clinical findings to the clinic. In addition, owing to their emerging role as plasma biomarkers, extracellular cathepsins are being considered as potential targets for non-invasive diagnostics [67]. In fact, we have successfully demonstrated the potential of plasma CTSD as a biomarker in NASH [12] as well as diabetic patients [68]. Next to extracellular cathepsins, in the current thesis we focused on alternate strategies to counteract cholesterol mediated-inflammation. When we employed a cholesterol mobilizing agent, $\mathrm{CD}$, we observed time-dependent pro-inflammatory effects despite beneficial effects on cholesterol sequestration (Chapter 6). Therefore, clinical application of CD, should be closely scrutinized and future studies should focus on optimizing therapeutic dose/time of $C D$ to prevent any proinflammatory side-effects.

Gut microbiota dysbiosis plays a crucial role not only in the pathogenesis of IBD but also in metabolic disorders such as NAFLD through its interactions via 'gut-liver' axis [65,69]. Consequently, microbiota intervention strategies such as supplementation of pre/probiotics and fecal microbiota transplantation (FMT) are being widely employed as treatment options for inflammatory disease therapy. FMT and probiotics were shown to improve lipid metabolism [70], insulin sensitivity and alleviation of steatohepatitis in rodent models [71]. Furthermore, adjuvant therapy targeting stress, particularly stress-induced inflammation, would be advantageous for the treatment of these diseases (Chapter 5).

Taken together, in this thesis we demonstrated various pharmacological and non-pharmacological treatment options aimed at modulating inflammation. We mechanistically demonstrated that pharmacological inhibition of extracellular CTSD activity might be a novel and safe drug target for NASH. Furthermore, we tested the cholesterol mobilizing CD as an alternative therapeutic strategy for metabolic inflammation and demonstrated some undesirable pro-inflammatory effects. Finally, we also reviewed the non-pharmacological stress management strategies in inflammatory disorders.

\section{Limitations and future perspectives}

In this thesis we validated putative targets such as extracellular CTSD and CD in preclinical rodent models of metabolic inflammation. While we found promising beneficial effects of these targets on disease outcomes in our studies, limitations include lack of validation of the findings in more preclinical models and in human samples. In view of the possible challenges in translating the pre-clinical findings to humans [72], the inhibitors need to be tested for their safety and tolerability for longterm use in humans. Therefore, further studies including human trials or/ and ex vivo models such as organ-on-chip platforms that reiterates the histologic endpoints in a single chip can serve as a robust non-invasive, humanized platform to study the disease course and to formulate effective therapeutics [73]. 


\section{Novel findings of this thesis}

In the present thesis, we aimed to provide translational insight into the underlying mechanisms that trigger inflammation in multiple diseases. The findings of the studies described in this thesis are highlighted below and summarized in Table1.

- Cathepsins exhibit both intra and extracellular functions in health and disease (Chapter 2).

- Extracellular cathepsin D plays a major role in lipid metabolism and insulin resistance during NAFLD progression (Chapter 3).

- Extracellular cathepsin D rather than intracellular fraction plays a key role in NASH mice with human-like lipoprotein profile and can be a novel target for modulating inflammation (Chapter 4).

- Stress impacts the inflammatory responses in IBD by acting on the complex gut/microbiota/brain axis (Chapter 5).

- 2-hydroxypropyl- $\beta$-cyclodextrin helps in cholesterol mobilization but shows time-dependent proinflammatory effects (Chapter 6 ).

Table 1: Summary of the major findings of this thesis along with limitations

\begin{tabular}{|c|c|c|c|}
\hline Chapter & Main findings & Limitations & Future directions \\
\hline Chapter 3 & $\begin{array}{l}\text { Inhibition of extracellular CTSD } \\
\text { reduced hepatic steatosis and } \\
\text { inflammation both in vitro and } \\
\text { in vivo }\end{array}$ & $\begin{array}{l}\text { The steatotic rat model used in this } \\
\text { study is an early model of } \\
\text { inflammation and does not mirror } \\
\text { hyperlipidemic profile as seen in } \\
\text { humans. }\end{array}$ & $\begin{array}{l}\text { To investigate the effects on } \\
\text { metabolic inflammation, a } \\
\text { more severe model of NAFLD } \\
\text { needs to be used. } \\
\text { Toxicity studies in pre-clinical } \\
\text { models need to be } \\
\text { performed. }\end{array}$ \\
\hline Chapter 4 & $\begin{array}{l}\text { Extracellular and intracellular } \\
\text { cathepsin D regulate distinct } \\
\text { metabolic pathways in NASH } \\
\text { and specific inhibition of } \\
\text { extracellular CTSD can be a safe } \\
\text { therapeutic target for NASH }\end{array}$ & $\begin{array}{l}\text { Small sample size for proteomics and } \\
\text { FACS. } \\
\text { Limited effects on inflammation. } \\
\text { Lack of validation in human models. }\end{array}$ & $\begin{array}{l}\text { Optimizing dose/time of } \\
\text { inhibitors. } \\
\text { Validation of the findings in } \\
\text { pre-clinical/ human models. } \\
\text { Pharmacokinetic studies. }\end{array}$ \\
\hline Chapter 6 & $\begin{array}{l}\text { 2-hydroxypropyl- } \beta \text {-cyclodextrin } \\
\text { helps in cholesterol } \\
\text { mobilization but elicits pro- } \\
\text { inflammatory responses in a } \\
\text { time-dependent fashion }\end{array}$ & $\begin{array}{l}\text { Lack of time-course experiments in } \\
\text { models of metabolic inflammation. } \\
\text { Lack of validation in human samples }\end{array}$ & $\begin{array}{c}\text { Perform dose/time } \\
\text { dependent experiments in } \\
\text { human samples. }\end{array}$ \\
\hline
\end{tabular}




\section{References}

1. Hotamisligil, G.S., Inflammation, metaflammation and immunometabolic disorders. Nature, 2017. 542(7640): p. 177185.

2. $\quad$ Li, C., et al., Macrophage polarization and meta-inflammation. Transl Res, 2018. 191: p. 29-44.

3. Maxfield, F.R. and I. Tabas, Role of cholesterol and lipid organization in disease. Nature, 2005. 438(7068): p. 612-21.

4. Viaud, M., et al., Lysosomal Cholesterol Hydrolysis Couples Efferocytosis to Anti-Inflammatory Oxysterol Production. Circ Res, 2018. 122(10): p. 1369-1384.

5. Spann, N.J. and C.K. Glass, Sterols and oxysterols in immune cell function. Nat Immunol, 2013. 14(9): p. 893-900.

6. van den Brink, W., et al., Current and Future Nutritional Strategies to Modulate Inflammatory Dynamics in Metabolic Disorders. Front Nutr, 2019. 6: p. 129.

7. Duan, Y., et al., Inflammatory Links Between High Fat Diets and Diseases. Front Immunol, 2018. 9: p. 2649.

8. Gibson, M.S., N. Domingues, and O.V. Vieira, Lipid and Non-lipid Factors Affecting Macrophage Dysfunction and Inflammation in Atherosclerosis. Front Physiol, 2018. 9: p. 654.

9. Houben, T., et al., Blood-derived macrophages prone to accumulate lysosomal lipids trigger oxLDL-dependent murine hepatic inflammation. Sci Rep, 2017. 7(1): p. 12550.

10. Tekin, I.O., A. Orem, and R. Shiri-Sverdlov, Oxidized LDL in inflammation: from bench to bedside. Mediators Inflamm, 2013. 2013: p. 762759.

11. Bieghs, V., et al., Trapping of oxidized LDL in lysosomes of Kupffer cells is a trigger for hepatic inflammation. Liver Int, 2013. 33(7): p. 1056-61.

12. Walenbergh, S.M., et al., Plasma cathepsin D correlates with histological classifications of fatty liver disease in adults and responds to intervention. Sci Rep, 2016. 6: p. 38278.

13. Samie, M.A. and H. Xu, Lysosomal exocytosis and lipid storage disorders. J Lipid Res, 2014. 55(6): p. 995-1009.

14. Li, W., X.M. Yuan, and U.T. Brunk, OxLDL-induced macrophage cytotoxicity is mediated by lysosomal rupture and modified by intralysosomal redox-active iron. Free Radic Res, 1998. 29(5): p. 389-98.

15. Benes, P., V. Vetvicka, and M. Fusek, Cathepsin D--many functions of one aspartic protease. Crit Rev Oncol Hematol, 2008. 68(1): p. 12-28.

16. Uchiyama, Y., Autophagic cell death and its execution by lysosomal cathepsins. Arch Histol Cytol, 2001. 64(3): p. 23346.

17. Conus, S., et al., Caspase-8 is activated by cathepsin $D$ initiating neutrophil apoptosis during the resolution of inflammation. J Exp Med, 2008. 205(3): p. 685-98.

18. Johansson, A.C., et al., Cathepsin $D$ mediates cytochrome $c$ release and caspase activation in human fibroblast apoptosis induced by staurosporine. Cell Death Differ, 2003. 10(11): p. 1253-9.

19. Vetvicka, V., et al., Procathepsin D and cancer: From molecular biology to clinical applications. World J Clin Oncol, 2010. 1(1): p. 35-40.

20. Hausmann, M., et al., Cathepsin $D$ is up-regulated in inflammatory bowel disease macrophages. Clin Exp Immunol, 2004. 136(1): p. 157-67.

21. Ketterer, S., et al., Cathepsin D deficiency in mammary epithelium transiently stalls breast cancer by interference with mTORC1 signaling. Nat Commun, 2020. 11(1): p. 5133.

22. Wolf, M., et al., Cathepsin D specifically cleaves the chemokines macrophage inflammatory protein-1 alpha, macrophage inflammatory protein-1 beta, and SLC that are expressed in human breast cancer. Am J Pathol, 2003. 162(4): p. 1183-90.

23. Goncalves, I., et al., High levels of cathepsin D and cystatin B are associated with increased risk of coronary events. Open Heart, 2016. 3(1): p. e000353.

24. Menzel, K., et al., Cathepsins B, L and D in inflammatory bowel disease macrophages and potential therapeutic effects of cathepsin inhibition in vivo. Clin Exp Immunol, 2006. 146(1): p. 169-80.

25. Khalkhali-Ellis, Z. and M.J. Hendrix, Two Faces of Cathepsin D: Physiological Guardian Angel and Pathological Demon. Biol Med (Aligarh), 2014. 6(2).

26. Moss, C.X., J.A. Villadangos, and C. Watts, Destructive potential of the aspartyl protease cathepsin D in MHC class IIrestricted antigen processing. Eur J Immunol, 2005. 35(12): p. 3442-51.

27. Mordente, J.A., et al., Hydrolysis of androgen receptor by cathepsin D: its biological significance in human prostate cancer. Br J Urol, 1998. 82(3): p. 431-5.

28. Nunn, S.E., D.M. Peehl, and P. Cohen, Acid-activated insulin-like growth factor binding protein protease activity of cathepsin D in normal and malignant prostatic epithelial cells and seminal plasma. J Cell Physiol, 1997. 171(2): p. 196204.

29. Morikawa, W., et al., Angiostatin generation by cathepsin D secreted by human prostate carcinoma cells. J Biol Chem, 2000. 275(49): p. 38912-20.

30. Mutka, A.L., et al., Murine cathepsin D deficiency is associated with dysmyelination/myelin disruption and accumulation of cholesteryl esters in the brain. J Neurochem, 2010. 112(1): p. 193-203.

31. Bae, E.J., et al., Haploinsufficiency of cathepsin D leads to lysosomal dysfunction and promotes cell-to-cell transmission of alpha-synuclein aggregates. Cell Death Dis, 2015. 6: p. e1901. 
32. Steinfeld, R., et al., Cathepsin D deficiency is associated with a human neurodegenerative disorder. Am J Hum Genet, 2006. 78(6): p. 988-98.

33. Follo, C., et al., Knock-down of cathepsin D affects the retinal pigment epithelium, impairs swim-bladder ontogenesis and causes premature death in zebrafish. PLoS One, 2011. 6(7): p. e21908.

34. Koike, M., et al., Cathepsin D deficiency induces lysosomal storage with ceroid lipofuscin in mouse CNS neurons. J Neurosci, 2000. 20(18): p. 6898-906.

35. Saftig, P., et al., Mice deficient for the lysosomal proteinase cathepsin D exhibit progressive atrophy of the intestinal mucosa and profound destruction of lymphoid cells. EMBO J, 1995. 14(15): p. 3599-608.

36. Handley, C.J., et al., Cathepsin D cleaves aggrecan at unique sites within the interglobular domain and chondroitin sulfate attachment regions that are also cleaved when cartilage is maintained at acid pH. Matrix Biol, 2001. 20(8): $\mathrm{p}$. 543-53.

37. van der Westhuyzen, D.R., W. Gevers, and G.A. Coetzee, Cathepsin-D-dependent initiation of the hydrolysis by lysosomal enzymes of apoprotein B from low-density lipoproteins. Eur J Biochem, 1980. 112(1): p. 153-60.

38. Baechle, D., et al., Cathepsin D is present in human eccrine sweat and involved in the postsecretory processing of the antimicrobial peptide DCD-1L. J Biol Chem, 2006. 281(9): p. 5406-15.

39. Zuhlsdorf, M., et al., Molecular forms of beta-hexosaminidase and cathepsin $D$ in serum and urine of healthy subjects and patients with elevated activity of lysosomal enzymes. Biochem J, 1983. 213(3): p. 733-40.

40. Leto, G., et al., Cathepsin D expression levels in nongynecological solid tumors: clinical and therapeutic implications. Clin Exp Metastasis, 2004. 21(2): p. 91-106.

41. Liu, L., et al., Increased Cathepsin D Correlates with Clinical Parameters in Newly Diagnosed Type 2 Diabetes. Dis Markers, 2017. 2017: p. 5286408.

42. Masson, O., et al., Pathophysiological functions of cathepsin D: Targeting its catalytic activity versus its protein binding activity? Biochimie, 2010. 92(11): p. 1635-43.

43. Rochefort, H. and E. Liaudet-Coopman, Cathepsin D in cancer metastasis: a protease and a ligand. APMIS, 1999. 107(1): p. 86-95.

44. Fusek, M. and V. Vetvicka, Dual role of cathepsin D: ligand and protease. Biomed Pap Med Fac Univ Palacky Olomouc Czech Repub, 2005. 149(1): p. 43-50.

45. Glondu, M., et al., A mutated cathepsin-D devoid of its catalytic activity stimulates the growth of cancer cells. Oncogene, 2001. 20(47): p. 6920-9.

46. Bourne, L.C., et al., Non-oxidative modification of low density lipoprotein by ruptured myocytes. FEBS Lett, 1997. 414(3): p. 576-80.

47. Derocq, D., et al., Cathepsin D is partly endocytosed by the LRP1 receptor and inhibits LRP1-regulated intramembrane proteolysis. Oncogene, 2012. 31(26): p. 3202-12.

48. Oldoni, F., et al., Naturally Occurring Variants in LRP1 (Low-Density Lipoprotein Receptor-Related Protein 1) Affect HDL (High-Density Lipoprotein) Metabolism Through ABCA1 (ATP-Binding Cassette A1) and SR-B1 (Scavenger Receptor Class B Type 1) in Humans. Arterioscler Thromb Vasc Biol, 2018. 38(7): p. 1440-1453.

49. Xian, X., et al., LRP1 integrates murine macrophage cholesterol homeostasis and inflammatory responses in atherosclerosis. Elife, 2017. 6.

50. Zhu, X., et al., Macrophage ABCA1 reduces MyD88-dependent Toll-like receptor trafficking to lipid rafts by reduction of lipid raft cholesterol. J Lipid Res, 2010. 51(11): p. 3196-206.

51. Stewart, C.R., et al., CD36 ligands promote sterile inflammation through assembly of a Toll-like receptor 4 and 6 heterodimer. Nat Immunol, 2010. 11(2): p. 155-61.

52. Erdmann, S., et al., Inflammatory cytokines increase extracellular procathepsin $D$ in permanent and primary endothelial cell cultures. Eur J Cell Biol, 2008. 87(5): p. 311-23.

53. Vidak, E., et al., Cysteine Cathepsins and their Extracellular Roles: Shaping the Microenvironment. Cells, 2019. 8(3).

54. Lugering, N., et al., IL-10 synergizes with IL-4 and IL-13 in inhibiting lysosomal enzyme secretion by human monocytes and lamina propria mononuclear cells from patients with inflammatory bowel disease. Dig Dis Sci, 1998. 43(4): p. 70614.

55. Briozzo, P., et al., MCF7 mammary cancer cells respond to bFGF and internalize it following its release from extracellular matrix: a permissive role of cathepsin D. Exp Cell Res, 1991. 194(2): p. 252-9.

56. Briozzo, P., et al., In vitro degradation of extracellular matrix with $\mathrm{Mr} 52,000$ cathepsin $\mathrm{D}$ secreted by breast cancer cells. Cancer Res, 1988. 48(13): p. 3688-92.

57. Chen, F.W., C. Li, and Y.A. Ioannou, Cyclodextrin induces calcium-dependent lysosomal exocytosis. PLoS One, 2010. 5(11): p. e15054.

58. Hissa, B., et al., Membrane cholesterol removal changes mechanical properties of cells and induces secretion of a specific pool of lysosomes. PLoS One, 2013. 8(12): p. e82988.

59. Zidovetzki, R. and I. Levitan, Use of cyclodextrins to manipulate plasma membrane cholesterol content: evidence, misconceptions and control strategies. Biochim Biophys Acta, 2007. 1768(6): p. 1311-24.

60. Pralle, A., et al., Sphingolipid-cholesterol rafts diffuse as small entities in the plasma membrane of mammalian cells. J Cell Biol, 2000. 148(5): p. 997-1008.

61. Onishi, M., et al., Hydroxypropyl-beta-cyclodextrin spikes local inflammation that induces Th2 cell and $T$ follicular helper cell responses to the coadministered antigen. J Immunol, 2015. 194(6): p. 2673-82. 
62. Eslamparast, T., et al., Recent advances in dietary supplementation, in treating non-alcoholic fatty liver disease. World J Hepatol, 2015. 7(2): p. 204-12.

63. Green, N., et al., A Review of Dietary Therapy for IBD and a Vision for the Future. Nutrients, 2019. 11(5).

64. Duff, W., et al., Non-pharmacological therapies for inflammatory bowel disease: Recommendations for self-care and physician guidance. World J Gastroenterol, 2018. 24(28): p. 3055-3070.

65. Ratziu, V., Z. Goodman, and A. Sanyal, Current efforts and trends in the treatment of NASH. J Hepatol, 2015. 62(1 Suppl): p. S65-75.

66. Bromme, D. and F. Lecaille, Cathepsin $K$ inhibitors for osteoporosis and potential off-target effects. Expert Opin Investig Drugs, 2009. 18(5): p. 585-600.

67. Kramer, L., D. Turk, and B. Turk, The Future of Cysteine Cathepsins in Disease Management. Trends Pharmacol Sci, 2017. 38(10): p. 873-898.

68. Ding, L., et al., Plasma cathepsin D activity is negatively associated with hepatic insulin sensitivity in overweight and obese humans. Diabetologia, 2020. 63(2): p. 374-384.

69. Jiang, X., et al., Advances in the Involvement of Gut Microbiota in Pathophysiology of NAFLD. Front Med (Lausanne), 2020. 7: p. 361

70. Alves, C.C., et al., Prebiotic and Synbiotic Modifications of Beta Oxidation and Lipogenic Gene Expression after Experimental Hypercholesterolemia in Rat Liver. Front Microbiol, 2017. 8: p. 2010.

71. Zhou, D., et al., Total fecal microbiota transplantation alleviates high-fat diet-induced steatohepatitis in mice via beneficial regulation of gut microbiota. Sci Rep, 2017. 7(1): p. 1529.

72. Hackam, D.G. and D.A. Redelmeier, Translation of research evidence from animals to humans. JAMA, 2006. 296(14): p. 1731-2.

73. Gori, M., et al., Investigating Nonalcoholic Fatty Liver Disease in a Liver-on-a-Chip Microfluidic Device. PLoS One, 2016. 11(7): p. e0159729. 
Summary 



\section{Summary}

The focus of this thesis is to increase the understanding of metabolic inflammatory processes underlying chronic inflammatory disorders with a goal to identify novel and safe therapeutic approaches.

Chapter 1 provides a comprehensive summary on the role of inflammation in the context of chronic inflammatory diseases such as NAFLD and IBD. Thereafter, the relationship between disturbed lipid metabolism and lysosomal enzymes in inflammation is described. Subsequently, the significance of non-pharmacological and pharmacological intervention strategies for treating inflammatory disorders are discussed. Finally, the aim and the outline of the thesis are summarized.

Chapter 2 gives an overview on the expression and functional aspects of lysosomal cathepsins. Next, the triggers and the respective mechanisms involved in the secretion of cathepsins into cytosol and extracellular space are described. In addition, detailed pathophysiological role of cathepsins in and outside of the lysosomes are discussed, followed by comprehensive information on the innovative experimental and clinical trials involving extracellular cathepsins.

Chapter 3 investigates the role of extracellular cathepsin D in lipid metabolism and insulin resistance associated with NAFLD. In this chapter, we tested a specific small-molecule extracellular cathepsin D inhibitor in both in vitro and in vivo models. Extracellular inhibitor-treated macrophages and HepG2 cells showed reduced levels of inflammation and improved cholesterol metabolism. Upon administration of the aforementioned inhibitor, high-fat fed Sprague-Dawley rats displayed reduced hepatic steatosis, inflammation and insulin resistance compared to controls. Collectively, this chapter demonstrates the causal role of extracellular CTSD in pathogenesis of NAFLD.

Chapter $\mathbf{4}$ offers translational insight into the respective roles of intra and extracellular fractions of cathepsin $D$ in NASH. To mimic a human hyperlipidemic profile, Ldlr-/- mice were fed a high- fat, high-cholesterol diet for a period of 10 weeks. Simultaneously, these mice were injected with either control/intra or extracellular smallmolecule inhibitors of cathepsin D. Extracellular CTSD inhibitor-treated mice showed reduced hepatic lipid accumulation, increased faecal bile acid levels and decreased inflammatory responses compared to intracellular CTSD inhibitor-treated mice. Proteomic analysis revealed that extra- and intracellular CTSD fractions regulate distinct metabolic pathways. We concluded that specific inhibition of extracellular CTSD inhibition is beneficial over intracellular CTSD in treating NASH.

Chapter 5 surveys the link between inflammation and gut/microbiota/brain axis in the context of IBD. Using evidence from several pre-clinical and clinical studies, the two-way relationship between gut microbiota and stress is addressed. Impact of non-pharmacological stress reduction strategies on the quality of life in IBD patients is evaluated.

Chapter 6 assesses the function of cholesterol-mobilizing agent, 2-hydroxypropyl- $\beta$-cyclodextrin (CD) in treating metabolic inflammation. To obtain an extreme model of inflammation, Ldlr-/- mice were transplanted with bone marrow from $\mathrm{Npc}^{\text {nih }}$ mice and were a fed high- fat, high-cholesterol diet for a period of 12 weeks. These mice were injected with $C D$ in the final three weeks. Compared to control mice, $N p c 1^{\text {nih }}$-tp mice that received $C D$ showed increased cholesterol mobilization with an unexpected increase in hepatic inflammation. In line with these in vivo results, a series of in vitro results confirmed the inflammatory effect of $C D$, which turned out to be time dependent.

Chapter $\mathbf{7}$ discusses the major outcomes of this thesis, with respect to the current knowledge in the field and highlights the clinical implications of the results. 


\section{Nederlandse Samenvatting}

De focus van dit proefschrift is het vergroten van het begrip van metabole inflammatoire processen die ten grondslag liggen aan chronische inflammatoire aandoeningen met als doel het identificeren van nieuwe en veilige therapeutische benaderingen.

Hoofdstuk 1 geeft een uitgebreide samenvatting van de rol van ontsteking in de context van chronische ontstekingsziekten zoals NAFLD en IBD. Daarna wordt de relatie tussen verstoord lipidenmetabolisme en lysosomale enzymen bij ontstekingen beschreven. Vervolgens wordt het belang van niet-farmacologische en farmacologische interventiestrategieën voor de behandeling van inflammatoire aandoeningen besproken. Tot slot worden het doel en de omvang van het proefschrift samengevat.

Hoofdstuk 2 geeft een overzicht van de expressie en functionele aspecten van lysosomale cathepsinen. Vervolgens worden de triggers en de respectievelijke mechanismen die betrokken zijn bij de uitscheiding van cathepsinen in het cytosol en de extracellulaire ruimte beschreven. Daarnaast wordt de gedetailleerde pathofysiologische rol van cathepsinen in en buiten de lysosomen wordt besproken, gevolgd door uitgebreide informatie over de innovatieve experimentele en klinische onderzoeken met extracellulaire cathepsinen.

Hoofdstuk 3 onderzoekt de rol van extracellulair cathepsine D in het lipidenmetabolisme en insulineresistentie geassocieerd met NAFLD. In dit hoofdstuk hebben we een specifieke kleinmoleculige extracellulaire cathepsine D-remmer getest in zowel in vitro als in vivo modellen. De met extracellulaire remmer behandelde macrofagen en HepG2-cellen vertoonden verminderde ontstekingsniveaus en verbeterd cholesterolmetabolisme. Na toediening van de bovengenoemde remmer vertoonden de met een hoog vetgehalte dieet gevoerde Sprague-Dawley-ratten verminderde leversteatose, ontsteking en insulineresistentie in vergelijking met controles. Gezamenlijk demonstreert dit hoofdstuk een causale rol van extracellulaire CTSD in de pathogenese van NAFLD.

Hoofdstuk 4 biedt translationeel inzicht in de respectievelijke rollen van intra- en extracellulaire fracties van cathepsine D in NASH. Om een humaan hyperlipidemisch profiel na te bootsen, kregen Ldlr-/-muizen gedurende een periode van 10 weken een vetrijk dieet met een hoog cholesterolgehalte. Tegelijkertijd werden deze muizen geïnjecteerd met ofwel controle / intra- of extracellulaire kleinmoleculige remmers van cathepsine D. De met extracellulaire CTSD-remmer behandelde muizen vertoonden verminderde hepatische accumulatie van lipiden, verhoogde fecale galzuur niveaus en verminderde inflammatoire responsen in vergelijking met intracellulaire CTSDremmer behandelde muizen. Proteomische analyse onthulde dat extra- en intracellulaire CTSDfracties verschillende metabole routes reguleren. We concludeerden dat specifieke remming van extracellulaire CTSD-remming gunstig is boven intracellulaire CTSD bij de behandeling van NASH.

Hoofdstuk 5 onderzoekt het verband tussen ontsteking en darm/microbiota/hersen-as in de context van IBD. Met behulp van bewijs uit verschillende preklinische en klinische onderzoeken wordt de tweerichtingsrelatie tussen darmmicrobiota en stress aangepakt. De impact van nietfarmacologische stressreductiestrategieën op de kwaliteit van leven bij IBD-patiënten wordt geëvalueerd. 
Hoofdstuk 6 beoordeelt de functie van het cholesterol-mobiliserend middel, 2-hydroxypropyl- $\beta$ cyclodextrine $(C D)$ bij de behandeling van metabole ontsteking. Om een extreem ontstekingsmodel te verkrijgen, werden Ldlr-/- muizen getransplanteerd met beenmerg van Npc1 in-muizen en kregen ze gedurende een periode van 12 weken een dieet met een hoog vetgehalte en een hoog cholesterolgehalte. Deze muizen werden in de laatste drie weken met CD geïnjecteerd. Vergeleken met controlemuizen vertoonden Npc1 in-tp-muizen die CD kregen een verhoogde cholesterolmobilisatie met een onverwachte toename van leverontsteking. In lijn met deze in vivo resultaten bevestigde een reeks in vitro resultaten het inflammatoire effect van $C D$, dat tijdsafhankelijk bleek te zijn.

Hoofdstuk 7 bespreekt de belangrijkste resultaten van dit proefschrift, met betrekking tot de huidige kennis in het veld en benadrukt de klinische implicaties van de resultaten. 



\section{Impact Paragraph}





\section{Impact paragraph}

\section{Social-economical and clinical relevance}

Chronic low-grade inflammation is a critical factor in progression of metabolic diseases including type 2 diabetes, NASH and cardiovascular diseases. Chronic inflammatory disorders have been identified as the leading cause of death worldwide today and their prevalence is going to steadily increase in the coming years. In European countries, the forecasted prevalence of NASH is $29.5 \%$ by 2030 [1]. Moreover, NASH and IBD patients incur substantial financial costs besides poor quality of life. In Europe, the annual healthcare costs incurred by IBD patients were estimated to be around $€ 5.6$ billion [2], while those related to NAFLD were estimated to be $€ 35$ billion [3] placing high economic burden on the society.

The shortage of early markers that can accurately differentiate NASH from steatosis and the lack of detailed mechanistic knowledge on NASH progression are the main obstacles in diagnosing and treating NASH, making liver transplantation the only therapeutic resort [4]. Although recent years have shown advancements in non-invasive imaging, to date, no (cost)effective methods are available to establish novel non-invasive diagnostic tools and therapeutic targets in the context of NAFLD. New pipelines of drugs should not only focus on reversing steatohepatitis but should also prove to be nontoxic and economical.

The current thesis demonstrated the specific inhibition of extracellular CTSD activity to be a novel strategy for the prevention and treatment of NASH. Even though cathepsin inhibitors are being extensively investigated for their therapeutic application in cancer and bone disorders, most clinical trials involving these inhibitors are abandoned due to their off-target effects [5]. In contrast to this, the targeted cathepsin inhibition followed in this thesis is promising since it specifically blocks the extracellular cathepsin fraction while preserving the essential physiological functions of intracellular cathepsin fraction, thus limiting toxic side effects. This approach thus can decrease the costs spent in clinical development and increase the chances of success in clinical trials. Furthermore, these small molecules are valuable for both liver disease and obesity-related pathologies since extracellular CTSD inhibition improved metabolic parameters including insulin resistance and dyslipidemia. Since extracellular CTSD activity is markedly impaired in atherosclerosis, cancer and lysosomal storage diseases such as NPC1, the findings from the current thesis can be extended to such co-morbidities thereby further increasing the clinical relevance. In addition to its therapeutic role, plasma CTSD has enormous potential as an early non-invasive biomarker for $\mathrm{NASH}$, thus helping in early diagnosis and treatment of NASH.

Another exciting approach to alleviate lipid storage problems is to use cholesterol extracting compounds such as cyclodextrins, which are already being extensively evaluated as treatment options in Alzheimer's, atherosclerosis, Krabbe and Niemann-Pick type C1 diseases [6]. Concomitant with the beneficial cholesterol-depleting effects, the current thesis uncovered the time-dependent pro-inflammatory effects of 2-hydroxypropyl- $\beta$-cyclodextrin $(C D)$ in several models of metabolic inflammation. While CD can help to reduce the disease burden in patients, our results warrant careful monitoring of $C D$ in clinic to prevent unwanted effects. These results thus have high clinical impact and pave a way to optimize time and dose regimes for future use of cyclodextrins. Greater affordability and improved safety of CDs can further augment their medical potential. 
Besides the above-mentioned contemporary therapeutic approaches, this thesis also discussed the importance of non-pharmacological stress-reduction strategies which can help in societal cost saving. Effective translation of these findings in larger patient populations can help draw more definite and detailed conclusions. Altogether, this endeavor can lead to reduction in socioeconomic burden and consequently improve quality of life in patients suffering from chronic inflammatory disorders.

\section{Novelty of the concept}

In this thesis, we investigated novel intervention strategies to treat metabolic inflammation. Along with our previous findings, the current thesis mechanistically demonstrated the pivotal role of cathepsin $D$ in lipid-induced hepatic inflammation. Using the state of the art in sillico approach, we designed and validated specific small-molecule inhibitors against cathepsin $D$ in a preclinical setting. Here, we show for the first time that specific inhibition of extracellular CTSD has beneficial effects in diminishing hepatic steatosis, inflammation and insulin resistance associated with NAFLD progression. Besides exploiting the function role of CTSD, we uncovered the potential mechanisms regulated by intra and extracellular CTSD fractions in the content of metabolic inflammation.

The benefits of suggested pharmacotherapies can be maximized by adhering to healthy lifestyle habits. While the information on lifestyle interventions for metabolic diseases is mostly limited to diet and exercise, this thesis consolidates the preclinical and clinical evidence on the role of stress reduction therapies in modulating inflammation that have enormous benefits in improving the quality-of-life in patients and reducing the healthcare burden. Also, this thesis provided critical data on the harmful pro-inflammatory effects of $C D$ in the context of cholesterol-induced metabolic inflammation, which provides a novel view on CD as therapeutic compound. As CD shuttles cholesterol directly from the trapped sites and has effective renal clearance, it is currently considered promising for patients who are intolerant to statin therapy or cannot maintain a low-calorie diet. As such, our novel observations challenge the clinical use of $C D$ and call for a careful reassessment of the drug's safety profile.

\section{Future perspectives and potential application}

Although the results in this thesis provide new diagnostic and therapeutic approaches for chronic inflammatory disorders, further work needs to be done to ensure that our findings are clinically translated to patients and the general public. In addition to other pre-clinical models, the results of the current project will be validated in blood-derived monocytes from healthy volunteers and samples from NASH. Since NASH patients have high risk of developing hepatocellular carcinoma ( $\mathrm{HCC})$, we planned to examine the cathepsin inhibitors in experimental models of HCC. As NASH shares the common pathogenic link with atherosclerosis [7], it is worthwhile to confirm and broaden the findings of the current thesis in atherosclerosis models. The next important step in human cathepsin research would be designing a personalized therapy with cathepsin inhibitors. For instance, optimizing the dosage of inhibitors by evaluating them in different cohorts would help a great deal in formulating efficient treatment regimens with even less side-effects [8].

Besides the role of CTSD, investigating the role of other lysosomal enzymes in the context of inflammation seems attractive. Several extracellular cathepsins are shown to mediate inflammatory 
responses by means of their proteolytic activity on the extracellular matrix components, chemokines, cytokines and different membrane receptors. In line, overexpression and secretion of cathepsins is one of the common denominators in several inflammation related pathologies including cancer, atherosclerosis, auto-immune diseases, and arthritis [9]. For instance, cathepsin B is a well-known prognostic marker for several cancers [10]. Moreover, CTSD secreted by breast cancer cells is known to degrade endogenous cysteine protease inhibitor, cystatin $C$ which subsequently leads to increased proteolytic activity of cathepsin B, thus exacerbating tumor progression and metastasis. Besides, blocking CTSD as well as CTSB/CTSL reduced inflammation in experimental colitis [11]. Therefore, it is reasonable to infer that extracellular cathepsins might synergistically contribute to the inflammatory responses. Future experiments with extracellular inhibitors of CTSB/L along with CTSD can clarify such speculations.

Lifestyle-induced weight loss and physical activity are highly recommended first line treatments for NASH patients. However, persistent lifestyle habits and weight loss are hard to reach and, unfortunately, lifestyle modifications alone are not effective in every individual. Nevertheless, it would be interesting to use combination treatment of the proposed pharmacological targets with the lifestyle interventions.

Lastly, instead of interfering with the inflammatory pathways, it is worthy to directly target the origin sites of inflammation, for example the gastrointestinal tract. High fat diet and obesity are known to disrupt the intestinal microflora composition and induce intestinal barrier dysfunction. Ultimately, these modifications lead to leaky mucosal barrier and release of bacterial metabolites that trigger a pro-inflammatory response leading to metabolic inflammation as seen in type 2 diabetes, NASH and atherosclerosis [12]. Therefore, it is worthwhile to study the gut microbiota-metabolism axis in the context of NASH. Collectively, the intricate relationship between lysosomal lipid metabolism and inflammation is gaining momentum and the therapeutic route for this field looks very promising. 


\section{References}

1. Estes, C., et al., Modeling the epidemic of nonalcoholic fatty liver disease demonstrates an exponential increase in burden of disease. Hepatology, 2018. 67(1): p. 123-133.

2. Cohen, R.D., et al., Systematic review: the costs of ulcerative colitis in Western countries. Aliment Pharmacol Ther, 2010. 31(7): p. 693-707.

3. O'Hara, J., et al., Cost of non-alcoholic steatohepatitis in Europe and the USA: The GAIN study. JHEP Rep, 2020. 2(5): p. 100142.

4. Wong, R.J., et al., Nonalcoholic steatohepatitis is the second leading etiology of liver disease among adults awaiting liver transplantation in the United States. Gastroenterology, 2015. 148(3): p. 547-55.

5. Kramer, L., D. Turk, and B. Turk, The Future of Cysteine Cathepsins in Disease Management. Trends Pharmacol Sci, 2017. 38(10): p. 873-898.

6. Braga, S.S., Cyclodextrins: Emerging Medicines of the New Millennium. Biomolecules, 2019. 9(12).

7. Bieghs, V., et al., NASH and atherosclerosis are two aspects of a shared disease: central role for macrophages. Atherosclerosis, 2012. 220(2): p. 287-93.

8. Palermo, C. and J.A. Joyce, Cysteine cathepsin proteases as pharmacological targets in cancer. Trends Pharmacol Sci, 2008. 29(1): p. 22-8.

9. Vizovisek, M., et al., Cysteine cathepsins as therapeutic targets in inflammatory diseases. Expert Opin Ther Targets, 2020. 24(6): p. 573-588.

10. Laurent-Matha, V., et al., Proteolysis of cystatin C by cathepsin D in the breast cancer microenvironment. FASEB J, 2012. 26(12): p. 5172-81.

11. Menzel, K., et al., Cathepsins $B, L$ and $D$ in inflammatory bowel disease macrophages and potential therapeutic effects of cathepsin inhibition in vivo. Clin Exp Immunol, 2006. 146(1): p. 169-80.

12. Tilg, H., et al., The intestinal microbiota fuelling metabolic inflammation. Nat Rev Immunol, 2020. 20(1): p. 40-54. 


\section{Acknowledgements}





\section{Acknowledgements}

"Why Maastricht?", one of the most asked questions in the last 4 years. To be honest, before March 2017, I had no clue that a place called Maastricht existed. The decision to relocate to Netherlands from Mumbai and finding a PhD position at Maastricht happened so fast and it's only fair to say that its destiny. Never have I regretted that decision or this place and I cannot be more grateful to its people. I would like to thank everyone who made my life and PhD journey way beyond memorable.

Firstly, I would like to thank to my promotor, Ronit for offering and supporting my PhD position. Ronit is someone you will instantly admire and never forget once you meet her. Her energy, charisma, and positive outlook are very contagious. I hope that I could be as lively, enthusiastic, and energetic as Ronit and to someday be able to run a team as efficient as hers. Ronit, thank you for being extremely caring and giving me the freedom to pursue various projects without objection. You always envision the bright side of things and motivate the team to finish projects with zero compromise on quality. Despite your crazy schedule, you always made time for us. At times when the project was not going as planned, you helped me find a way to navigate through it and finish the thesis on time. You encouraged and expected us to think more independently about our experiments and results. Besides being a great scientist and mentor, you are a wonderful mother. I was lucky to be your student and would love to come back and work with you in the future.

Next, thanks to my co-promoter Tom who has directed me from day one. Tom, you supervised me in the lab, helped me organize and perform experiments, assisted me with instrumentation, guided me in writing, correcting presentations, proof reading, tutoring, rebuttals (basically everything $;)$ ) You taught me every single detail from start of the experiment to processing the data. You work super hard and are extremely flexible in attending me and other PhDs. You made sure that we were progressing despite the tough situation with COVID. You never take slack and multitask efficiently. I enjoyed all the fun times, jokes, lab outings, conferences, dinners and online games that we participated together. Congratulations on all your accomplishments and all the very best for your future! Hope one day you will appreciate the spicy Indian food;)

I will forever be thankful to former colleague and one of my good friends, Yvonne who has been helpful in providing support and advice many times both personally and professionally. Yvonne, you always care and look out for people. In fact, you helped me with organizing the paperwork when I was still in India and made sure I got everything I wanted for home and at work after my arrival. Thank you for teaching many things in the lab and guiding me in writing. Your attention to detail, precision in performing experiments, an organized way of working in the lab are few of the many things that I will carry with me. I'll never forget the wonderful evenings and fun activities we've done together. I know I can always count on you, Joël and we love Gisèle the most.

I thank the members of my PhD assessment committee, Prof. dr. Marteen Honing, Dr. Jef Verbeek, Prof. dr. Martijn Brouwers, Dr. L.B. Creemers and Dr. Jan Theys for critically reviewing my thesis and granting its approval.

I thank my paranymphs Albert and Ines for being super cooperative throughout my PhD. Albert, thanks for agreeing to be my paranymph. You are knowledgeable in just about everything, helpful, and friendly. I am very grateful for your support, scientific advice and the many insightful suggestions you offered during my PhD. I enjoyed our brainstorming sessions, debates, and long days in the lab. Thanks for fixing my careless mistakes in the lab. I wish you the very best Albert! Dearest Ines, my 
fellow minion, great officemate and the group party planner, you always bring a cheerful vibe to the group. Thank you for sharing everything from pipette tips to makeup tricks! Thanks for all your help in the past years, especially with mice experiments. Thoroughly enjoyed the chitchat, book reviews, movie nights, dinners and lab outings. Will miss your laughter and singing. All the best for your promotion. Wish you and Bogdan loads of success and happiness.

A good support system is important to surviving and staying sane during PhD. I was lucky to be a part of one we call "metabolism masters". Firstly, Ines and Lingling, you guys formed the core of my research time, and I couldn't have survived without you. We've all been there for one another and have taught ourselves and each other many things. Lingling, I miss you and wish you continued working here. We had so much fun together in and outside of the lab. I can talk to you about everything under the sun and you would patiently listen. I would like to visit Mia as soon as I can. I wish you, Lichuan and Mia a wonderful life ahead. Dennis, you are the coolest and most generous technician one can ask for! (The YES man!) Thank you for putting up with us in the lab, help organize experiments and being the quality control. It was always fun sharing the lab with you. Annemarie, you are a hardworking student and very responsive in answering questions. Thanks for all your help in planning cathepsin projects. Good luck with your PhD! The youngest member of the group, Mengyeng, you have been so friendly to me. Wish you the best for your PhD. You are the best teammates and I miss you a lot! Thanks to all the intern students during my time at Ronit's group for all the help in the lab and the fun.

I thank all the members at the department who helped me in one way or the other: Jan, thank you for warm welcome during the start of my PhD. Thank you for providing tips and suggestions during the lab chat. You listen to research presentations with great attention and offer helpful advice to all the students. Joost, it was always a pleasure to speak with you. Thank you for the scientific discussions during the lab meetings and guiding me with fractionation protocols. The way you interact with your students and your positive outlook are admirable. William, thank you for your feedback and helpful suggestions during the lab chat. You are extremely knowledgeable scientist. Petra, you are always very helpful and caring. Thank you for organizing departmental social gatherings and fun activities and taking great care of students. Also, thank you for all the administrative help these past months. I enjoyed chatting with you every time. Miranda, you are sweet, friendly and willing to help everyone! Thank you for your suggestions during labchat. I enjoyed all the social events we attended. Li-Yen, you are always friendly and cheerful about everything. Thank you for all the food and for introducing me to other expats. Wish you and Stein the best! Pierre, my white Indian friend;) Thank you for listening and giving me advice whenever I needed. I enjoyed our coffee breaks and lunch discussions.

Thanks to fellow PhDs: Francesco, a crazy Italian friend who curses a lot! Thank you for the numerous fun times during the last couple of years and filling MilliQ tanks ;). You are a great friend and success with your PhD. Also thank Adriana for all the fun evenings we spent together. Jobran, you are an extremely nice and helpful person in general, thank you for keeping me company in the office and at the parties. You will be a great father. Wish you and your wife lots of happiness! Myrthe, you are a hard-working student and a talented baker. Thank you for helping me with confocal microscopy and good luck with your PhD.

Marion, thank you for helping me with animal experiments, support with manuscripts and teaching many things related to staining and scoring sections. I appreciate all the guidance I received from 
you. Agnieszka, you greet me with a bright smile every morning. Thank you for being very helpful in the lab, with ordering chemicals and supplies. I enjoyed our chat time. I thank Bieke and Monique for the advice and help in the lab. I enjoyed all the vlaai breaks and social activities with you all. A big thank you to everyone at the department who helped me along my PhD journey.

I also thank the former members of the Department: Will, thank you for introducing me to lab etiquettes. Ilvy, thank you for your help in the lab during the first years of my PhD. Mieke, thank you for your advice regarding staining protocols and its always a pleasure to meet you in social events. Thanks to Shujin and Aomin for being wonderful officemates. You guys were so much fun, and I missed you a lot during my last year.

I thank all my collaborators for their wonderful support. Special thanks to colleagues from M4I in helping us with omics experiments. Berta, thank you for your help in analyzing the data and proofing manuscript. I am intrigued by your scientific insight. Thanks to your wonderful team, especially Ronny and Isabeau for their great efforts in conducting experiments and in data analysis. I also extend my thanks to Aten Porus team in India. Princy, Aditya and Sandeep, thank you for your support at various stages of the project.

I thank Nederlandse Vereniging voor Hepatolgie (NVH) for providing financial support in printing this thesis.

Huge thanks to Alkmaar family, the colleagues and friends at PALLAS, ICHOS and INVAP for being super nice and supportive to our long distance relationship. Thank you for making me a part of your dinner events, activities, and family gatherings.

I thank my friends (too many to list here but you know who you are!) for providing support and friendship that I needed.

I thank my parents, my brother and sister-in-law. Thank you for providing so much love and care. I love you so much, and I would not have made it this far without you. Mom and dad, I miss you dearly and hate the fact that you cannot travel and be here with me. Special thanks to my in-laws, my cutest nephew Aarav and his wonderful parents who all have been supportive and caring.

Finally, thanks to my husband, Deepak: aka my highway man, the love of my life, my anchor and the most annoying but sweetest husband. Deepak, thank you for taking care of me, loving me unconditionally, for travelling miles so I can stay close to Uni, for being my chauffeur and hosting many fun evenings. Thanks to your wannabe cocktails, I made better friends ;) You are my biggest critic and greatest admirer. Your quirkiness and sense of humour (THE wife jokes!!), hiking appetite and racing madness kept me sane. These past years have not been an easy ride (albeit in a BMW), both academically and personally. I thank you from the bottom of heart for sticking by my side, even when I was irritable. Sorry for all the cancelled plans, but I am looking forward (or not!) for all the mountain trails awaiting us! May the force be with us! 



\section{Curriculum Vitae}





\section{Curriculum Vitae}

Tulasi Yadati was born in 1990 in Kadapa, India. In 2010. She obtained her Bachelors degree from Osmania University (Hyderabad, India) with specialization in genetics and microbiology. Later, she completed the Level-II certification in Protein modelling and rational drug designing from GVK Biocampus and Accelyrs. In 2011, she joined the Masters program at the Department of Animal Biotechnology, University of Hyderabad, India. Her senior year project was focused on characterizing the three-dimensional organization of Whey acidic protein (Wap) gene locus in mouse mammary epithelial cells. She graduated in 2013 as university topper for which she was awarded the INSPIREJunior Research Fellowship from Department of Science and technology, India which allowed her to pursue research studies in the lab of Prof. Aparna Dutta Gupta for a period of two years where she was engaged in investigating the functional role of Hexamerin proteins in the rice moth morphogenesis.

In July 2017 she joined the group of Prof. Ronit Shiri-Sverdlov for her doctoral studies at Maastricht University. Her research mainly focused on investigating the mechanistic role of lysosomal enzymes in lipid-induced metabolic inflammation and preclinical validation of small-molecule drugs targeted at several aspects of metabolic inflammation. The results of the work are published in this thesis.

Tulasi is currently working as a postdoctoral researcher under the supervision of Prof. dr. Stan Van de Graaf, at Tytgat Institute for Liver and Intestinal Research, Amsterdam University Medical Center, The Netherlands. 


\section{List of Publications}

1. Khurana P, Yadati T, Goyal S, Dolas A, Houben T, Oligschlaeger Y, Agarwal AK, Kulkarni A, ShiriSverdlov R (shared first author). Inhibiting Extracellular Cathepsin D Reduces Hepatic Steatosis in Sprague-Dawley Rats. Biomolecules. 2019 May 4;9(5):171. doi: 10.3390/biom9050171.

2. Oligschlaeger Y, Yadati T, Houben T, Condello Oliván CM, Shiri-Sverdlov R. Inflammatory Bowel Disease: A Stressed "Gut/Feeling". Cells. 2019 Jun 30;8(7):659. doi: 10.3390/cells8070659.

3. Yadati T, Houben T, Bitorina A, Shiri-Sverdlov R. The Ins and Outs of Cathepsins: Physiological Function and Role in Disease Management. Cells. 2020 Jul 13;9(7):1679. doi: 10.3390/cells9071679.

4. Yadati T, Houben T, Bitorina A, Oligschlaeger Y, Gijbels M, Mohren R, Lütjohann D, Khurana P, Goyal S, Kulkarni A, Theys J, Cillero-Pastor B, Shiri-Sverdlov R; Inhibition of extracellular cathepsin $D$ reduces hepatic lipid accumulation and leads to mild changes in inflammation in NASH mice. Frontiers in Immunology. DOI=10.3389/fimmu.2021.675535 (Nominated for Young Hepatologist Award, 2021).

5. Houben T, Yadati T, de Kruijf R, Gijbels M, Luiken J, Zandvoort M, Kapsokalyvas D, Lütjohann D, Westerterp M, Plat J, Leake D, Shiri-Sverdlov R (shared first author); Pro-Inflammatory Implications of 2-Hydroxypropyl- $\beta$-cyclodextrin Treatment. Frontiers in Immunology. DOI=10.3389/fimmu.2021.716357

6. Bitorina AV, Oligschlaeger Y, Ding L, Yadati T, Westheim A, Houben T, Vaes RDW, Olde Damink SWM, Theys J, Shiri-Sverdlov R. OxLDL as an Inducer of a Metabolic Shift in Cancer Cells. J Cancer. 2021 Aug 3;12(19):5817-5824. doi: 10.7150/jca.56307.

7. Ahmad F, Mitchell RD, Houben T, Palo A, Yadati T, Parnell AJ, Patel K, Shiri-Sverdlov R, Leake DS. Cysteamine Decreases Low-Density Lipoprotein Oxidation, Causes Regression of Atherosclerosis, and Improves Liver and Muscle Function in Low-Density Lipoprotein Receptor-Deficient Mice. J Am Heart Assoc. 2021 Sep 21;10(18):e017524. doi: 10.1161/JAHA.120.017524. 


\section{Oral Presentations}

1. February 2018: Dutch Liver Science Retraite (Nederlandse Vereniging voor Hepatolgie), Spier, the Netherlands

Title: Inhibiting extracellular Cathepsin D reduces hepatic inflammation in steatohepatitis.

2. February 2018: Research Line 2 lunch meeting, Maastricht, the Netherlands

Title: Inhibiting extracellular Cathepsin D reduces hepatic inflammation in steatohepatitis.

3. March 2018: European Fatty Liver Conference, Vaals, The Netherlands (awarded the 2 nd best pitch presentation)

Title: Unconventional treatment for a conventional disease: role of extracellular cathepsin $D$ in NASH.

4. March 2018: Nederlandse Lipoproteinen Club, Amsterdam, The Netherlands

Title: Inhibiting extracellular Cathepsin D reduces hepatic inflammation in steatohepatitis.

5. June 2018: Seminar meeting, Genetics and Cell Biology, Maastricht, The Netherlands

Title: "Therapeutic potential of extracellular cathepsin D in Non-alcoholic steatohepatitis (NASH)

6. February 2019: Dutch Liver Science Retraite (Nederlandse Vereniging voor Hepatolgie), Spier, the Netherlands (awarded the 3rd best oral presentation)

Title: Extracellular Cathepsin D as a novel target for NAFLD

7. October 2020: Research Line 2 lunch meeting, Maastricht, the Netherlands

Title: Extracellular cathepsin D as a target for Non-alcoholic steatohepatitis (NASH)

\section{Poster Presentations}

1. November 2018: Annual NUTRIM symposium, Maastricht University, The Netherlands.

Title: "Extracellular cathepsin D: A new target for NAFLD?"

2. November 2019: Annual NUTRIM symposium, Maastricht University, The Netherlands.

Title: "Circulating cathepsin D improves hepatic lipid metabolism in murine NASH." 